\title{
A STUDY ON SLIDING MODE STATE ESTIMATION
}

\author{
By \\ JINHEE CHOI \\ Bachelor of Science in Engineering \\ Seoul National University \\ Seoul, Korea \\ 1979 \\ Master of Science in Engineering \\ Korea Advanced Institute of Science and Technology \\ Seoul, Korea \\ 1981
}

Submitted to the Faculty of the Graduate College

of the Oklahoma State University

in partial fulfillment of the requirements

for the Degree of

DOCTOR OF PHILOSOPHY

May, 1993 


\section{COPYRIGHT}

b y

CHOI, JINHEE

May 1993 


\section{OKIAHOMA STATE UNIVERSITY}

A STUDY ON SLIDING MODE

STATE ESTIMATION

Thesis Approved:

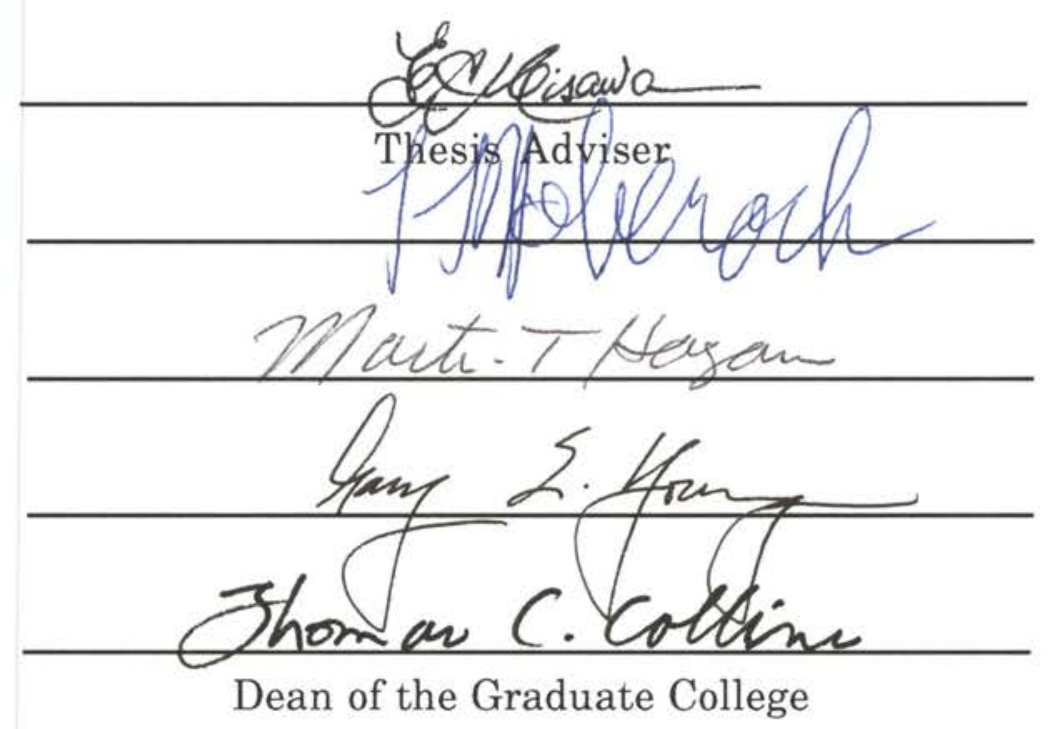




\section{ACKNOWLEDGMENTS}

I would like to express my sincere appreciation to my advisor, Dr. Eduardo A. Misawa, for his excellence in guiding and encouragement throughout the course of this research. I am indebted to him for his valuable suggestions and inspiration. I would also like to thank Dr. Gary E. Young, Dr. Lawrence L. Hoberock and Dr. Martin T. Hagan for their helpful comments and discussions.

To my parents, JongKwan Choi and KeumSoon Kim, my deepest thanks for their immeasurable love. My special thanks go to my wife HeeWon for her sacrifice and praying for me. Also, I am deeply grateful to my parents in law and our pastors for their praying.

I want to thank the financial supports provided by the Republic of Korea. 


\section{TABLE OF CONTENTS}

Chapter

Page

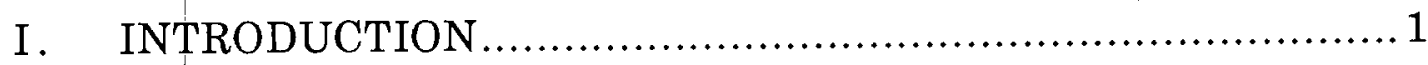

1.1 Overview ....................................................... 1

1.1 .1 Scope ....................................................... 1

1.1.2 Contributions of the Thesis..............................2

1.1.3 Problem Definition ...................................... 3

1.2 Literature Survey ............................................. 4

1.2.1 Nonlinear Observer ..................................... 4

1.2.2 Stability Theory and Robust Controls ..................... 7

1.3 Differential Equations with Discontinuous

Right-Hand Side ........................................ 8

1.3.1 1-order Examples ........................................... 8

1.3.3 2-order Examples .......................................10

II. FUNDAMENTALS OF SLIDING OBSERVERS...................14

2.1 Systems and Problem Statement ..............................14

2.2 Solutions of Discontinuous Differential Equations ...........16

2.2.1 Sliding and Passing Conditions ..........................16

2.2 .2 Switching System ......................................18

2.3 Sliding Observer Dynamics ................................20

2.3.1 Reaching Dynamics ...................................20

2.3.2 Sliding Dynamics .....................................22

2.4 Coordinate Transformation.................................23

2.4.1 Shifted-coordinate System .............................23

2.4.2 Sliding Observer Error Dynamics in the Shifted-coordinate...................................25

2.4.3 Passing Points on the Hyperplane ......................28 
III. STABILITY ANALYSIS …........................................

3.1 Worst-case Analysis .......................................... 31

3.1.1 Worst-case Analysis of Switching System...............31

3.1.2 Example..................................................35

3.2 Worst-Direction in the shifted-coordinate ....................37

3.2.1 2-Dimension Reaching Dynamics .......................37

3.2.2 Worst Direction in the Velocity Field.....................38

3.2.3 Numeric Search..........................................41

3.2.4 Approximate Worst Direction ...........................43

3.2.5 General Remarks.....................................49

3.3 Lyapunov-like Function ....................................49

3.3.1 Introduction .............................................49

3.3.2 Lyapunov-like Function Theorem......................50

3.3.3 Passing Jump of Lyapunov-like Function................53

3.4 Lyapunov-like Stability Analysis .............................56

3.4.1 Lyapunov-like Stability Theorem ........................56

3.4.2 Contour of Lyapunov-like Function.....................69

IV . DESIGN OF SLIDING OBSERVERS ...........................75

4.1 Reaching Dynamics Response .............................75

4.1.1 Reaching Dynamics Response .........................75

4.1.2 Conceptual Comparisons of Stability Theorems ........78

4.1.3 Sliding Observer Design Algorithm....................79

4.2 Comparative Example with VSS Observer.....................81

4.2.1 Sliding Observer........................................81

4.2.2 VSS Observer......................................... 83

4.3 Inverted Pendulum........................................... 85

4.4 Nonlinear Mass-Spring System with Friction.................88

4.5 Super-tanker Lateral Dynamics.............................92

V. ROBOT CONTROL BASED ON SLIDING OBSERVERS ............97

5.1 Sliding Observer for Multiple Measurement.................97 
5.2 Robots Dynamics .........................................99

5.2.1. Dynamics of Rigid Robots .............................99

5.2 .2 Two link Manipulator................................. 100

5.3 The Case with No Parameter Uncertainty ................... 103

5.3.1 The Design of the Sliding Observer .................... 103

5.3.2 Feedback Linearization Control ........................ 105

5.4 The Case with Parameter Uncertainty....................... 108

5.4.1 The Design of the Sliding Observer .................... 108

5.4 .2 Adaptive Control ....................................... 109

5.4 .3 Sliding Control...................................... 112

VI. STOCHASTIC SLIDING OBSERVER DESIGN .................. 115

6.1 Introduction ............................................... 115

6.2 Sliding Observer Design for Noisy Measurements..........116

6.3 1st Order System Example..................................... 119

6.4 2nd Order System Example ................................. 121

6.5 Prediction and Simulation.................................. 123

6.5.1 Effect of Measurement Noise ........................... 123

6.5.2 Effect of Process Noise ..................................... 125

6.5.3 Effect of Parametric Mismatch ........................ 127

VII. CONCLUSION AND FUTURE RESEARCH .................... 130

7.1 Summary and Conclusion............................... 130

7.1 .1 Thesis Summary....................................... 130

7.1 .2 Conclusion ........................................... 132

7.2 Future Research ......................................... 133

7.2.1 Design Algorithm by Lyapunov-like Stability .......... 133

7.2.2 Worst-case Lyapunov-like Function Search ........... 135

7.2.3 Design procedure Based on the Lyapunov-like Stability Theorem .................................... 136

7.2.4 Design Algorithm for Noisy Measurement............. 137

7.2.5 Using Nonlinear Terms in the Observer .............. 137 
BIBLIOGRAPHY ..................................................... 139

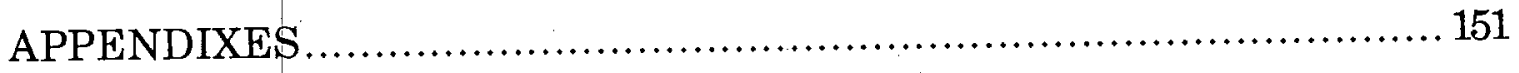

APPENDIX A - MATHEMATICAL BACKGROUND ............ 154

APPENDIX B - STABILITY THEOREMS ...................... 159

APPENDIX C - SIGN EQUALIZATION OF PASSING

STATES........................................ 174 


\section{LIST OF FIGURES}

Figure

Page

1.1 Comparison between linear and nonlinear systems.................. 9

1.2 Luenberger observer error dynamics .................................10

1.3 Sliding observer error dynamics....................................10

1.4 Plotting of sign and saturation function .............................11

1.5 A linear system with high gains ...................................11

2.1 Shifted-coordinate and sliding patch...............................26

2.2 Passing points on the hyperplane .....................................27

3.1 Worst-case analysis in 2-dimensional phase plane ...................32

3.2 Stable region by the stability criterion .................................34

3.3 The templet (dotted lines) and disturbed trajectories ...................36

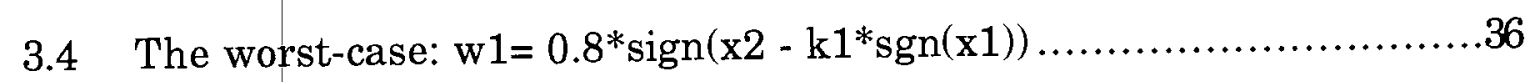

3.5 Velocity field and the worst trajectory .................................37

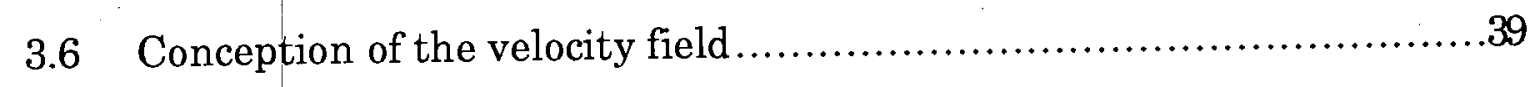

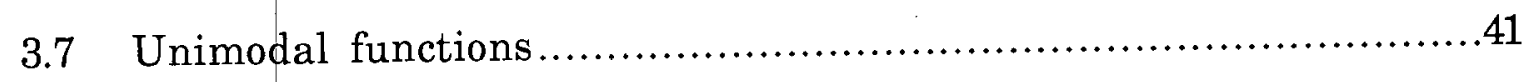

3.8 Block diagram of the optimization algorithm ........................42

3.9 The simulation results of the numeric search.........................43

3.10 The trajectory of solution point and velocity field.......................45

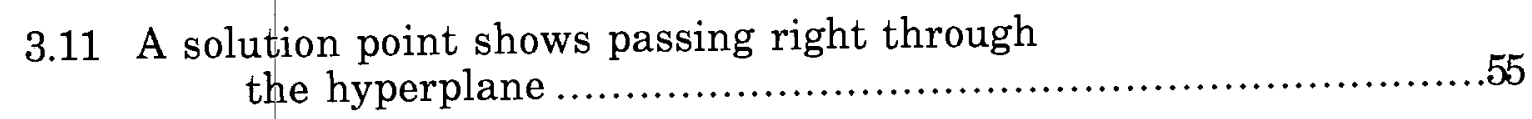

3.12 Passing points on the hyperplane .....................................57 
Figure

Page

3.13 Phase plane and Lyapunov-like function .............................61

3.14 Phase plane and Lyapunov-like function ..............................64

3.15 Equivalent eigenvalues...............................................64

3.16 Phase plane and Lyapunov-like function ..............................68

3.17 Contour of Lyapunov-like functions ..................................70

3.18 Contour of Lyapunov-like function on the hyperplane .................71

3.19 Contour of large Lyapunov-like function case..........................71

3.20 Contour of small Lyapunov-like function case .........................72

3.21 Contour of badly designed case .........................................73

3.22 Contour of suggested case .............................................

4.1 Conceptual comparison of stability theorems .........................77

4.2 Lyapunov-like function contour on the hyperplane ......................78

4.3 Simulation results of SOON .......................................... 83

4.4 Sliding observer simulation results ..................................83

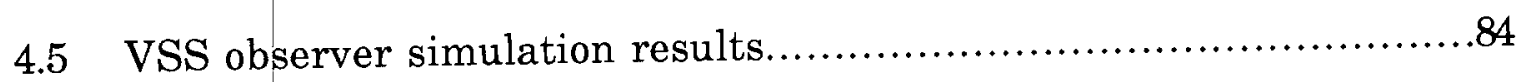

4.6 Inverted pendulum with a moving support...........................85

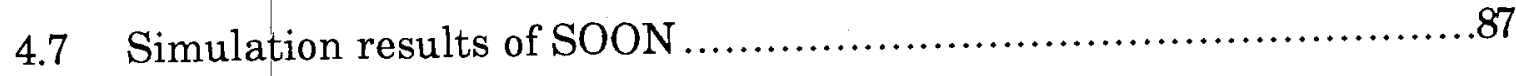

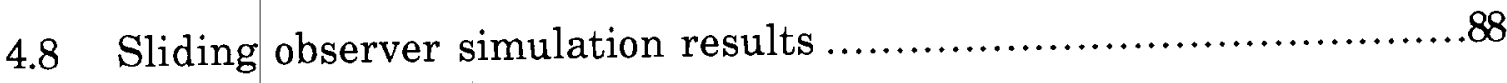

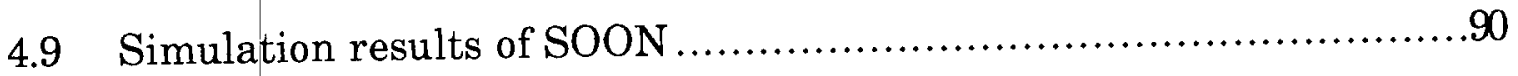

4.10 Simulation results: time domain plot of 1 st states....................90

4.11 Simulation results: time domain plot of 2 st states.....................91

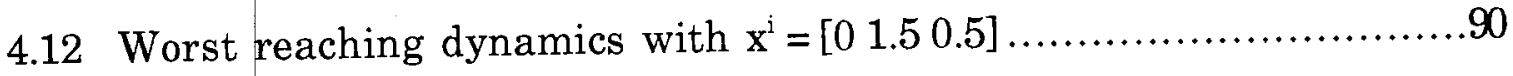

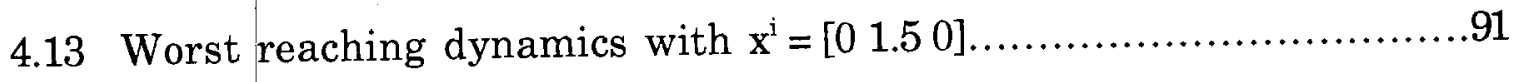

4.14 The actual and estimated states of super-tanker......................95 
Figure

Page

5.1 Two link Manipulator .............................................. 101

5.2 The simulated results of the sliding observer ....................... 105

5.3 The simulated results of feedback linearization control

based on the sliding observer.................................... 107

5.4 The simulated results of feedback linearization control based on the sliding observer with uncertain parameters.............. 109

5.5 The simulated results of adaptive control based on the sliding observer with uncertain parameter.................. 111

5.6 The simulated results of sliding control based on the sliding observer with uncertain parameter....

6.1 Effect of measurement noise: $\mathrm{SO}$ vs. $\mathrm{KF}$

(for the 1st order system).

6.2 Effect of measurement noise: $\mathrm{SO}$ vs. $\mathrm{KF}$

(for the 2nd order system) 124

6.3 Effect of process noise: SO vs. $\mathrm{KF}$ (for the 1st order system) ........... 126

6.4 Effect of process noise: SO vs. KF (for the 2nd order system) ......... 126

6.5 Effect of parameter mismatch: $\mathrm{SO}$ vs. $\mathrm{KF}$

(for the 1st order system).

6.6 Effect of parameter mismatch: $\mathrm{SO}$ vs. $\mathrm{KF}$

(for the 2-order system)

7.1 Lyapunov-like function and the passing jump...................... 134

7.2 Conception of the velocity field and Lyapunov-like function........... 135

A.1 The velocity field near the hyperplane ................................ 153

A.2 Fourier series expansion of square wave and signum function ...... 154

A.4 A saturation function.............................................. 157

A.3 Differential of the Fourier series of square wave .................... 155

B.1 The equivalent gain profile ......................................... 161

B.2 Estimation error dynamics........................................ 163 
Figure $\quad$ Page

C.1 Transient responses of the reaching dynamics ...................... 177

C.2 The conceptual diagram of the reaching time ........................ 178

C.3 Convexity and concavity of the function ............................. 186

C.4 Convexity of the state and Sign change of the state.................. 187 


\section{NOMENCLATURE}

A Upper diagonal system matrix

$A_{m} \quad$ Meta system matrix

$A^{\circ} \quad$ Nominal system value

$\widetilde{\mathrm{A}}$

Difference between the system and nominal system

$\mathrm{c}_{\mathrm{ij \textrm {k }}}$

Coriolis and centrifugal terms

$\mathrm{C}_{\mathrm{s}} \quad$ Fictitious step advancing constant.

$\mathrm{d}_{\mathrm{kj}}$

Coefficient of inertia matrix $D(q)$

g

Nonlinear vector function,

g

Gravitational constant: $9.8 \mathrm{~m} / \mathrm{sec}^{2}$

$h^{*}$

Kalman filter optimal gain

$\mathrm{H}$

Linear correction coefficient: $\mathrm{H}^{\mathrm{T}}=\left[\mathrm{h}_{1}^{\mathrm{T}}, \mathrm{h}_{2}^{\mathrm{T}}, \ldots, \mathrm{h}_{\mathrm{n}}^{\mathrm{T}}\right], \quad \mathrm{H} \in \mathcal{R}^{\mathrm{n} \times \mathrm{m}}$

K

Switching coefficient: $\mathrm{K}^{\mathrm{T}}=\left[\mathrm{k}_{1}^{\mathrm{T}}, \mathrm{k}_{2}^{\mathrm{T}}, \ldots, \mathrm{k}_{\mathrm{n}}^{\mathrm{T}}\right], \quad \mathrm{K} \in \mathbb{R}^{\mathrm{n} \times \mathrm{m}}$

$\mathrm{K}_{\mathrm{s}} \quad$ Shifted switching coefficient

$N_{1} \quad$ RIDF of $1_{s}$ and the function of statistics of $\widetilde{y}$

$\mathrm{P}_{2^{*}} \quad$ Fictitious advancing point

$\mathrm{P}$

Unique positive-definite solution to the Lyapunov equation.

$p_{i}$

Right eigenvector of A associated with $\lambda_{\mathrm{i}}$.

$\mathrm{q}_{\mathrm{i}}$

Left eigenvector of A associated with $\lambda_{i}$.

$q_{i}$

Generalized coordinate that is the joint angle

$\mathrm{q}_{\mathrm{r}}$

Reference velocity 


\begin{tabular}{|c|c|}
\hline Q & Process noise intensity \\
\hline $\mathrm{R}$ & Measurement noise intensity \\
\hline $\mathbf{s}$ & Signs:,+- or 0 \\
\hline $\mathrm{s}^{*}$ & Different sign from $\mathrm{s}$ : $\operatorname{sign}(\mathrm{s}) \neq \operatorname{sign}\left(\mathrm{s}^{*}\right)$ \\
\hline $\mathbf{s}_{+}^{i}\left(\tau_{j}\right)$ & Initial state on the hyperplane at time $\tau_{j}$ \\
\hline$\dot{\mathrm{s}}_{\mathrm{i}}$ & Time derivative of sliding surface \\
\hline $\mathrm{u}_{\mathrm{d}}$ & Sliding observer error dynamics disturbance input \\
\hline $\mathrm{u}$ & Control input vector \\
\hline $\mathrm{v}$ & Measurement noise vectors \\
\hline $\mathrm{w}$ & Input or disturbance input \\
\hline W & $\mathrm{W}=\{0, \ldots, 0 \mathrm{w}\}^{\mathrm{T}}$ \\
\hline$x_{+}$ & Shifted coordinate $\left(\mathrm{x}_{1}>0\right)$ \\
\hline $\mathrm{x}$. & Shifted coordinate $\left(\mathrm{x}_{1}<0\right)$ \\
\hline$x^{i}$ & Initial state at the passing point. \\
\hline $\mathrm{y}$ & Measurement vector \\
\hline$\widetilde{\mathrm{y}}$ & Output error vector \\
\hline $\mathrm{Y}(\mathrm{q}, \dot{\mathrm{q}}, \ddot{\mathrm{q}})$ & Regressor and $\mathrm{q}$ is an $\mathrm{r}$-order vector of parameters. \\
\hline $\mathbf{z}$ & System state vector \\
\hline$\delta$ & Rudder angle (degree) \\
\hline$\delta\left(\mathrm{x}_{1}\right)$ & Dirac delta function \\
\hline$\delta \xi$ & Magnitude of velocity difference \\
\hline$\phi_{\mathrm{k}}$ & Gravitational forces and torques \\
\hline$\Phi$ & State transition matrix: $\Phi=\operatorname{diag}(\mathrm{Q}, \mathrm{R})$ \\
\hline
\end{tabular}


$\theta$

System parameter

$\sigma_{+}^{i}\left(\tau_{j}\right) \quad$ Initial state on the hyperplane in the shifted coordinate

$\begin{array}{ll}\tau_{\mathrm{i}} & \text { Control inputs } \\ w & \text { Process noise vectors }\end{array}$

$\Omega_{0} \quad$ Sliding zone in the hyperplane $\left(\mathrm{x}_{1}=0\right.$ and $\left.\left|\mathrm{x}_{2}\right| \leq \mathrm{k}_{1}\right)$

$\Omega_{+} \quad$ Right half space $\left(\mathrm{x}_{1}>0\right)$

$\Omega \quad \quad$ Left half space $\left(\mathrm{x}_{1}<0\right)$

$\psi \quad$ Yaw angle (degree)

$\dot{\psi} \quad$ Yaw rate (degree/sec) 


\section{CHAPTER I}

\section{INTRODUCTION}

\subsection{Overview}

\subsubsection{Scope}

A considerable amount of work has been done in the field of state estimation since the late forties. For nonlinear systems, the observers have mainly relied on the precise knowledge of the plant. Recently, state estimation of nonlinear system in the presence of uncertainty has attracted much attention. This attention, of course, is due to the fundamental importance of the issue. There are several robust observers for the uncertain systems, for example, sliding observers, VSS observers, adaptive observers etc. [Misawa, 1989]. However, choosing a proper observer is not easy because, in many cases, the observer design is problem-dependent.

Recently, price-reduced and powerful microprocessors have applied to nonlinear estimation techniques that require on-line calculations. However, the estimation schemes, for example, with the computation of the coupled covariance and filter equations are burdensome on applicable capabilities of the microprocessors in practice. Hence, this study aims at developing a simple structure observer so that it can be implemented using microprocessors. 


\subsubsection{Contributions of the Thesis}

For nonlinear/uncertain systems, adaptive and robust observers have been proposed. Since the robustness of the variable structure system is known, nonlinear observers using "sliding mode" technique have been studied in several ways. An approach using the dual concept of the variable structure system requires the so-called "matching condition.", Misawa et al., proposed a sliding observer that can be designed without the matching condition. They proposed two design procedures: the first one' is for the case of the strictly positive real system and the other one is based on the absolute stability theorem. With the absolute stability theorem, the sliding observer utilizes a saturation function instead of a sign function.

This study focuses on the robust features of the sliding observer by introducing a Lyapunov-like function theorem. The main works are summarized as follows: first, the fundamentals of the sliding observer were reviewed and explained systematically for the robust features including the shearing effects. For the stability of the observer, the worst case was analyzed so that an algebraic stability condition was derived for the 2-order case. By introducing a coordinate transformation, it is found that the sliding observer can be interpreted in the light of a linear system theory. Stability theorems combined this coordinate transformation and the Lyapunov-like theorem were proved for the stability of the observer. Consequently, a robust nonlinear observer design algorithm, "Sliding Observer design by wOrst reaching dynamics for Nonlinear/ uncertain system" (SOON) is developed for a phase variable canonical form system. Several comparative examples show the strong points of the sliding observer.

The sliding observer for multiple measurements was developed and 
applied to designing an observer based controller for a SCARA robot.

In order to realize the optimal Kalman-Bucy filter, we should know the exact intensities of noise which compose the state error covariance matrix, i.e., the Riccati equation. Practically, however, the noises are not measurable, and the noise intensities may be changed according to the variation of operating conditions. With the nominal statistical properties of the sensor noises, Misawa[1988] applied the method of statistical linearization in designing a suboptimal stochastic sliding observer. In his study, the method was applied to the first order Markov process. The extension from the first order Markov process of the previous work [Misawa, 1988] to the second order Markov process was performed. The extension work includes the parameter mismatch cases in which the optimal solution of the Lyapunov equation was numerically obtained by the steep decent method.

\subsubsection{Problem Definition}

A nonlinear dynamic system can usually be represented by a set of nonlinear differential equations in this form:

$$
\begin{aligned}
& \dot{\mathrm{z}}=\mathrm{g}(\mathrm{z}, \mathrm{u}, \mathrm{t}, w, \theta) \\
& \mathrm{y}=\mathrm{h}(\mathrm{z}, \mathrm{u}, \mathrm{t}, \mathrm{v}, \theta)
\end{aligned}
$$

where $\mathrm{g}(\mathrm{z}, \mathrm{u}, \mathrm{t}, w, \theta)$ is an $\mathrm{nx} 1$ nonlinear vector function, and $\mathrm{z}$ is the internal state vector; $\mathrm{y}$ is the measurement vector, $\mathrm{u}$ is the control input vector, and $w$ and $\mathrm{v}$ are the process and measurement noise vectors. $\theta$ is the system parameter, and the system is explicitly time dependent.

A state estimation problem can be interpreted as a composition of a dynamical system that estimates the state $\mathrm{z}$ from the measurement $\mathrm{y}$ and possibly including the input $u$. In this study, we are interested in a simple 
and robust observer that will guarantee a priori stability and convergence with some relaxed knowledge of the system. Practically, the observer will be implemented using microprocessors or computers and will be corrupted with measurement noise and process noise. With this scope, the ideal state estimation problem can be described as designing a simple observer that can be implemented with desired accuracy and stability in the noise and uncertain environment. For practical situations, the class of problems will be specified. In the study, the plant is assumed to be described by systems of first order differential equations in the canonical form:

$$
\left\{\begin{array}{c}
\dot{\mathrm{z}}_{1}=\mathrm{z}_{2} \\
\dot{\mathrm{z}}_{2}=\mathrm{z}_{3} \\
\cdots \\
\dot{\mathrm{z}}_{\mathrm{n}-1}=\mathrm{z}_{\mathrm{n}} \\
\dot{\mathrm{z}}_{\mathrm{n}}=\mathrm{g}(\mathrm{z}, \mathrm{u}, \mathrm{t}, w, \theta) \\
\mathrm{y}(\mathrm{t})=\mathrm{C} \mathrm{z}(\mathrm{t})
\end{array}\right.
$$

where $\mathbf{z} \in \mathrm{R}^{\mathrm{n}}, \mathrm{y} \in \mathrm{z}_{1}, \mathrm{C} \in \mathrm{R}^{\mathrm{1xn}}$ and $\mathrm{g}(\mathrm{z}, \mathrm{u}, \mathrm{t}, w, \theta)$ is a nonlinear/uncertain function.

\subsection{Literature Survey}

A literature survey was performed on the nonlinear observers and the related fields such as stability theories and robust control. The nonlinear observer suryey includes a brief summary of Misawa and Hedrick's recent paper (see detail Misawa [1989]) and is updated with the papers published since 1989.

\subsubsection{Nonlinear Observers}

Since the cornerstone of modern estimation theories, the Wiener filter and the celebrated Kalman filter, the estimation theory has been a very vigorous research topic. In the case of a linear plant and a linear relationship 
between the unknown state and the noisy observations, the estimation problem has been solved by Kalman and Bucy [Kalman, 1960; Kalman, 1961]. Furthermore the Kalman filter is so easily installed in computers that it has been widely used.

In the non-linear case, the Extended Kalman filter [Gelb, 1974; Sorenson, 1985] has a analogous structure to the Kalman filter. The Extended Kalman filter uses both the nonlinear and linear models, and it assumes that the system is perfectly known. The computation of the coupled covariance and filter equation imposes considerable real-time computational efforts [Wishner, 1969; Misawa, 1989]. To avoid the computational load, the approximation of the Extended Kalman filter's residual-gain was precomputed in the Constant Gain Extended Kalman filter [Safonov, 1978; Safonov, 1980]. This method has the guaranteed robustness as the mathematical dual of the LQG controller. This method assumes that the system matrix is Gateaux differentiable [Holzman, 1970], nonanticipative, dynamical nonlinear operators with finite incremental gain so that has limitation on hard nonlinearities.

Phaneuf [1968] developed a Statistically Linearized filter [Gelb, 1974] that uses the describing function. It requires the probability density function to determine the describing function. Beaman [1984] suggests the new scheme to lessen the computational burden due to the error covariance equation. A priori performance and robustness cannot be guaranteed without exactly knowledge of the statistics of $\mathrm{x}$.

The concept of Global Linearization Methods was introduced by Bestle and Zeitz [1983] and extended to the multiple output case by Krener et al. [Krener, 1985; Walcott, 1987]. With the assumption of the existence of a nonlinear transformation $\mathrm{T}\left(\mathrm{x}^{*}\right)$ that in the new coordinate $\mathrm{x}^{*}$, the system 
may be transformed into a linear observer canonical form. Following the arguments involving the starting vector of Bestle and Zeitz [1983], Walcott et al., showed the necessary transformation for a single output case. This method transforms a system into a simple linear system but cannot guarantee the existence of the transformation.

Another approach is the Extended Linearization Method, which falls into the category of gain scheduling methods. Baumann and Rugh [1986] introduce an observer-design technique for non-linear systems that yields constant eigenvalues for the error differential equation linearized about fixed equilibrium points. The method of extended linearization requires that the system dynamics be known exactly, and this method cannot guarantee the performance and stability except in the neighborhood of constant operating points.

One major idea in designing the nonlinear observer is linearizing the system about a nominal trajectory so that the linear observer technique is applied. Another way to design the nonlinear observer is to use a transformation technique that expresses the system in observable canonical form that simplifies the design process.

However, most of the schemes of nonlinear observers have relied on an exact knowledge of the system. Recently, the robustness of observer for a nonlinear system has been pursued by using the dual concept of Variable Structure System [Drakunov, 1986; Slotine, 1987; Misawa, 1988; Misawa, 1989; Walcott, 1986; Walcott, 1987; Chen, 1987; Chen, 1990]. A nonlinear observer using the sliding mode technique was analyzed for a stochastic case by Drakunov [1986] and for a deterministic case by Slotine, Hedrick and Misawa [1986]. Another approach to estimation of uncertain nonlinear systems is the adaptive observer [Hori, 1988; Chen, 1988; Chen, 1990]. The 
drawbacks of the adaptive observer are that it requires the so-called matching conditions, and the estimation error cannot be guaranteed to converge but only to be bounded.

\subsubsection{Stability Theory and Robust Controls}

Several references can be found on the Lyapunov theory applied to nonlinear systems with discontinuous functions [Alimov, 1960, 1961; Bockman, 1991; Peleties, 1991; Stalford, 1981]. Passivity interpretations of adaptive control laws are discussed in the book of Landau [1979]. The reader is referred to Vidyasagar's book [1978] for a detailed discussion of absolute stability. The circle criterion and its extensions to non-autonomous systems were derived by Narendra [1973], and Zames [1966].

The idea of variable structure systems and sliding surfaces [Filippov, 1964] has been investigated mostly in the Soviet literature [Utkin, 1984; Utkin, 1977; Utkin, 1978; Itkis, 1976]. It is well known that VSS shows robustness, i.e., disturbance rejection property. The undesirable feature, chattering is remedied by plugging a boundary layer into a neighboring sliding surface [Slotine, 1983; Slotine, 1984]. The sliding observer is motivated by the dual concept of sliding control to give the inherent robustness to the nonlinear estimation. Plant uncertainty is the main reason why we need feedback. However, plant uncertainty was largely neglected by modern control [Bryson, 1969] during the 1960s and 1970s. The optimal controllers based on LQG have shown poor performance and even instability in real systems because of high sensitivity to modelling errors [Doyle, 1981; Doyle, 1982]. Beginning in the late 1970s, the study of robust control systems by means of singular-value analysis is represented by the work of investigators centered at the MIT. 
The earliest statement of the separation theorem in the literature of control theory was given by Joseph and Tou [Joseph, 1961] on discrete-time systems in 1961. The separation theorem for discrete-time systems was so reasonable and convenient that it was immediately adopted, without rigorous proof, for continuous-time systems [Friedland, 1986; Walcott, 1988]. It is well known that the separation principle cannot be applied directly to uncertain nonlinear systems. Esfandiari and Khalil [1989] used the singular perturbation technique to prove the stability of the observer-based closed-loop control in the presence of time-scaling.

The literature on the subject of computational considerations and robustness with model uncertainties [Safonov, 1978] is sparse. Schweppe [1973] discussed nonlinear estimation and provided a good intuitive understanding of the trade-off between computational requirements and residual-gain choice.

The references about the stochastic processes spread over a wide range of literature, according to the level of mathematical rigor. General textbooks [Papoulis, 1984; Friedland, 1986] are available. Jazwinski [1970] and Astrom [1970] give readable accounts of stochastic differential equations including the Ito and Straonovitch calculi. A higher level of mathematical rigor is found by Kushner [1967, 1984] and Anderson et al. [1986].

\subsection{Differential Equations with Discontinuous Right-Hand Sides}

\subsubsection{1-order Examples}

A linear time invariant system with a pole at -3 is a asymptotic stable and its nonzero initial state decays to origin exponentially. If a disturbance 
is applied, then the system shows state error as in Figure 1.1 (a). The linear system is

$$
\dot{\mathbf{x}}=-3 \mathbf{x}+w
$$

Let us consider a first-order differential equation with a discontinuous right-hand side.

$$
\dot{\mathbf{x}}=-3 \operatorname{sgn}(\mathbf{x})+w
$$

(a) -D LTI system: $x$ and $x$ with $w=2$ sqw(t/4)

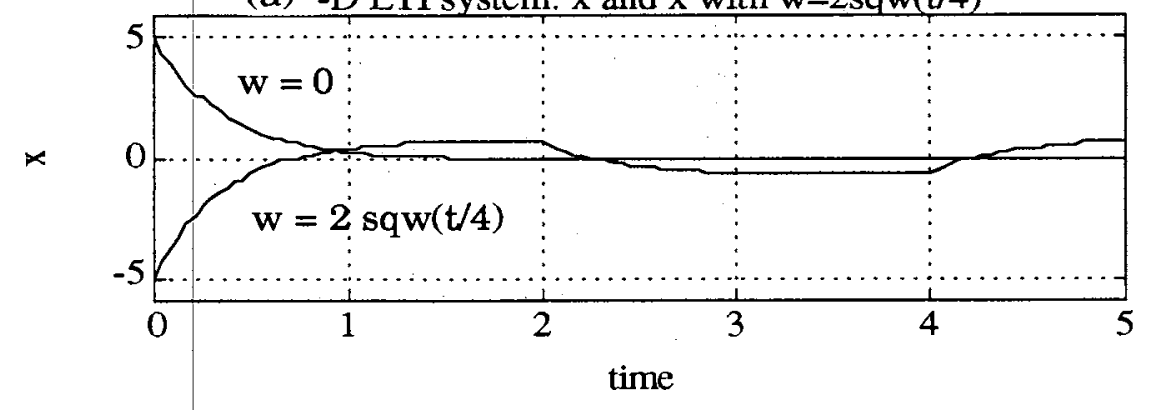

(b) -D SW system: $x$ and $x$ with $w=2 s q w(t / 4)$

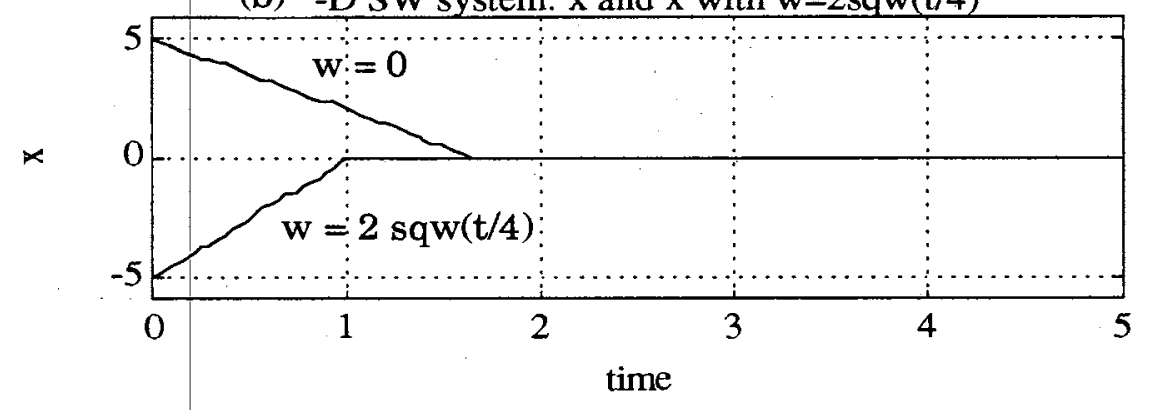

Figure 1.1 Comparison between linear and nonlinear systems

(a) Linear system (b) nonlinear switching system

Without the disturbance input, the switching system, with initial condition $x^{i}=5$, decays 3 per second. The approaching slope of this nonlinear system is constant, compared to the asymptotic behavior of the linear system. 
The velocity field of this system with the bounded disturbance $(|w|<3)$ is

$$
\left\{\begin{array}{l}
\dot{x}<0, \text { for } x>0 \\
\dot{x}>0, \text { for } x<0
\end{array}\right.
$$

This velocity field composes a sliding motion at the switching plane. With a disturbance less than the switching coefficient, this switching system does not leave the sliding surface. The robust feature of the system with a discontinuous right-hand side is mainly owing to this sliding motion. Until arriving at the switching plane $\mathrm{x}=0$, the signum function is constant. While staying in the switching plane, the instant time mean of the signum function has the value between $-1 \leq \zeta \leq 1$, i.e., from the Filippov's equivalent dynamics.

\subsubsection{2-order Examples}

Let us consider a nonlinear system that is a 2-order linear canonical form system with switching functions.

$$
\left\{\begin{array}{l}
\dot{\mathrm{x}}_{1}=-\mathrm{h}_{1} \mathrm{x}_{1}+\mathrm{x}_{2}-\mathrm{k}_{1} 1_{\mathrm{s}}\left(\mathrm{x}_{1}\right) \\
\dot{\mathrm{x}}_{2}=-\mathrm{h}_{2} \mathrm{x}_{1}-\mathrm{k}_{2} 1_{\mathrm{s}}\left(\mathrm{x}_{1}\right)+w
\end{array}\right.
$$

where $\mathrm{w}$ is a input or disturbance input and $\mathrm{h}_{1}: 2, \mathrm{~h}_{2}: 2, \mathrm{k}_{1}: 0.1, \mathrm{k}_{2}: 2, w=\sin (\mathrm{t})$, $1_{s}\left(x_{1}\right)=\operatorname{sign}\left(x_{1}\right)$ or $\operatorname{sat}\left(x_{1}\right)$

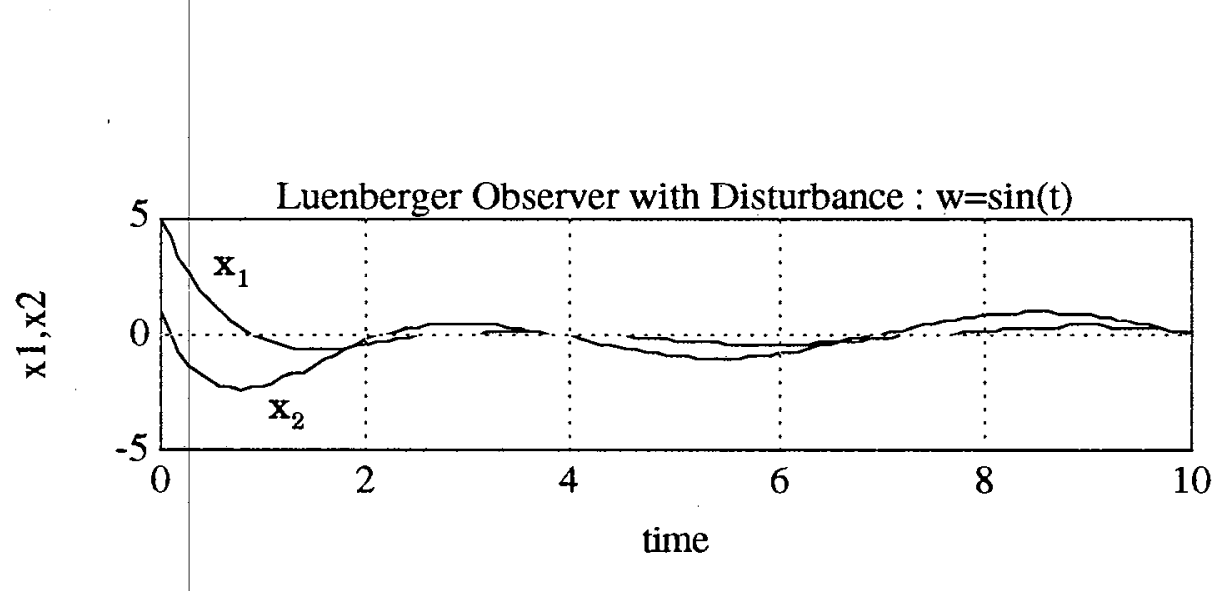

Figure 1.2 Luenberger observer error dynamics 
If the system has only linear correction terms, then it is the same as the error dynamics of the Luenberger observer. If switching terms are added to the system then it becomes a nonlinear sliding observer error dynamics.

When the disturbance is applied to the system, the linear system shows the state error according to the disturbances as shown in Figure 1.2. Compared to the linear system, the nonlinear system with additional switching terms compensates the disturbance and quickly diminishes state errors as in Figure 1.3. These properties are the robust features that is essential to observers.

We can see the sliding motion begins at approximately 4 seconds. The switching term looks like the control action of "pulse width modulation control." The saturation function looks like the control action of "amplitude modulation control" and it has the opposite sign of disturbance.
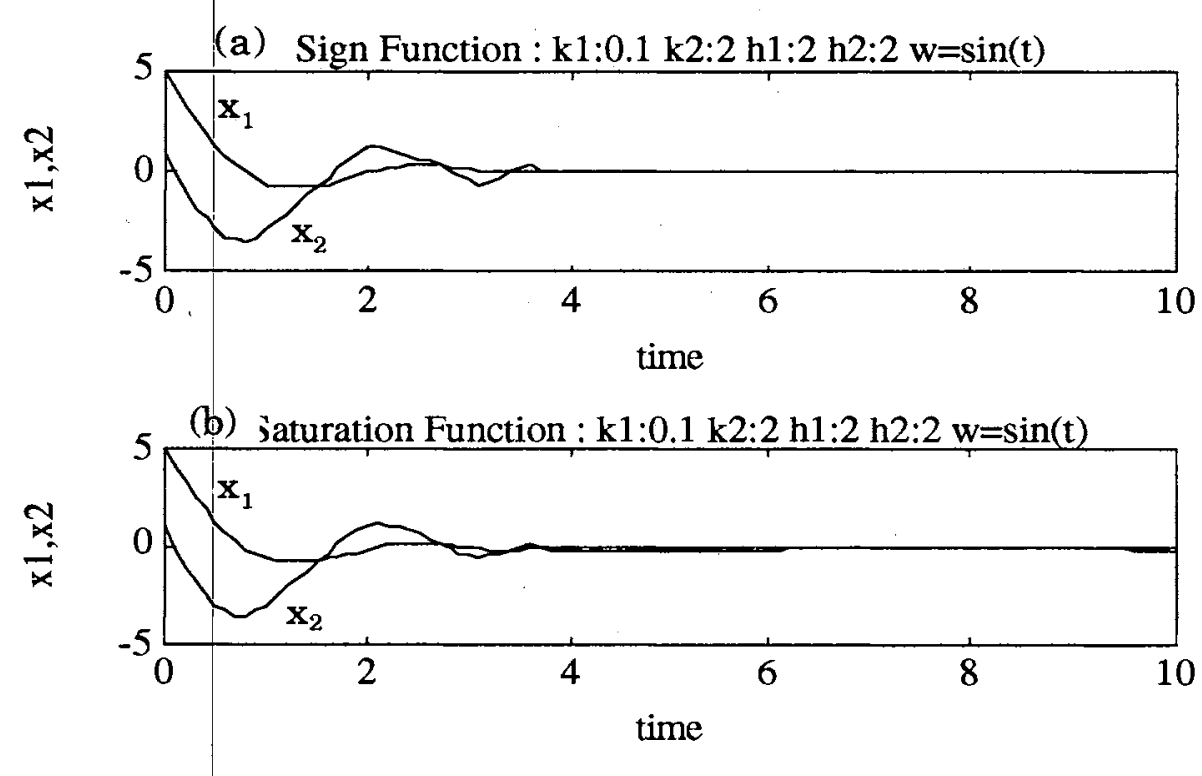

Figure 1.3 Sliding observer error dynamics

(a) Sign function case (b) Saturation function case 


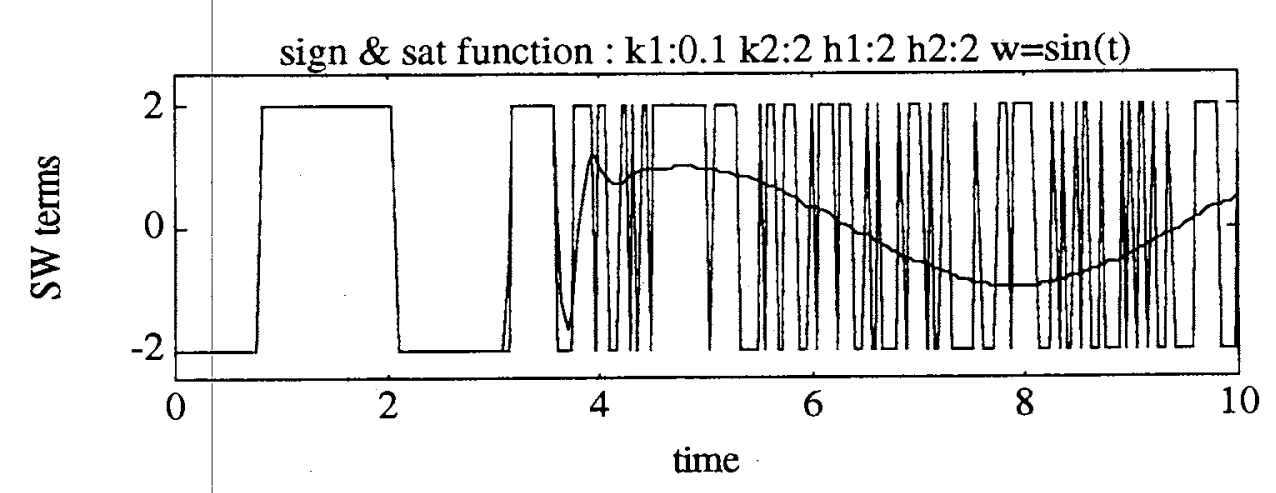

Figure 1.4 Plotting of sign and saturation function

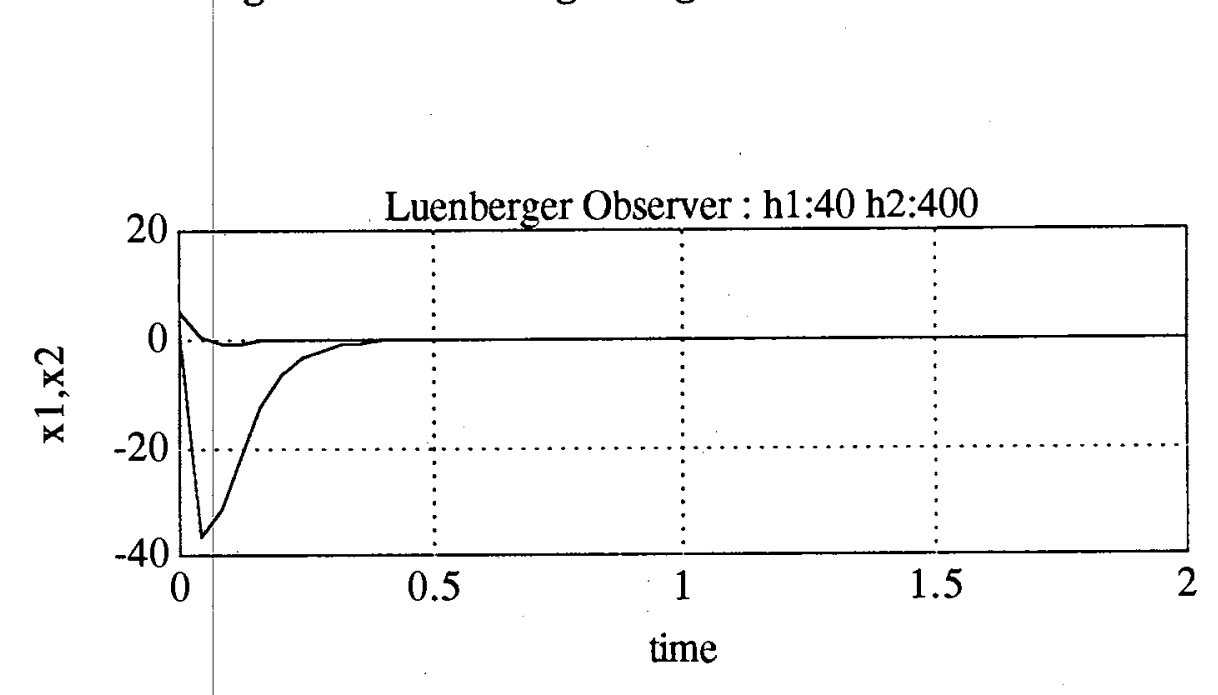

Figure 1.5 A linear system with high gains

The linear system with high gain has a big overshoot compared to the former ones. It is well known that the sensor noise is also amplified by the same gains. The signum function in the nonlinear system has "adaptive" features that behave as a high gain function near the origin and as a low gain function far from the origin.

A nonlinear observer that just had switching terms added to it shows desirable robust features. Since the signum function does not satisfy the Lipschitz condition, the stability of the system with the signum function has 
been usually studied by the Lyapunov stability theory. Hence, if the Lyapunov function is not found for the nonlinear observers with the signum function, then the nonlinear observers have been designed with quadratic Lyapunov function which required the matching condition [Walcott, 1986] or can be designed only for a strictly positive real system [Misawa, 1988]. This study aims developing a sliding observer without the matching condition. 


\section{CHAPTER I I}

\section{FUNDAMENTALS OF SLIDING OBSERVERS}

\subsection{Systems and Problem Statement}

It is assumed that the plant is described by a set of first order differential equations in the canonical form:

$$
\left\{\begin{array}{c}
\dot{\mathrm{z}}_{1}=\mathrm{z}_{2} \\
\dot{\mathrm{z}}_{2}=\mathrm{z}_{3} \\
\cdots \\
\dot{\mathrm{z}}_{\mathrm{n}-1}=\mathrm{z}_{\mathrm{n}} \\
\dot{\mathrm{z}}_{\mathrm{n}}=\mathrm{g}(\mathrm{z}, \mathrm{u}, \mathrm{t}, \mathrm{w}, \theta) \\
\mathrm{y}(\mathrm{t})=\mathrm{C} \mathrm{z}(\mathrm{t})
\end{array}\right.
$$

where $\mathbf{z} \in \mathbf{R}^{n}, \mathbf{y} \in \mathbf{R}^{m}, \mathrm{C} \in \mathbf{R}^{\mathrm{m} \times n}, \mathrm{~g}(\mathrm{z}, \mathrm{u}, \mathrm{t}, w, \theta)$ is a nonlinear/uncertain function of the state, input, time, disturbance, and system parameter. With this system, the sliding observer is suggested as follows:

$$
\dot{\mathrm{z}}=\left\{\begin{array}{c}
\hat{\mathrm{z}}_{2} \\
\vdots \\
\hat{\mathrm{z}}_{\mathrm{n}} \\
\hat{\mathrm{g}}
\end{array}\right\}+\mathrm{H}(\mathrm{y}-\mathrm{C} \hat{\mathrm{z}})+\mathrm{K} \mathrm{I}_{\mathrm{s}}(\tilde{\mathrm{y}})
$$

where $\quad \widetilde{y}=y-C \hat{z}$

$$
\begin{aligned}
& \mathrm{H}^{\mathrm{T}}=\left[\mathrm{h}_{1}^{\mathrm{T}}, \mathrm{h}_{2}^{\mathrm{T}}, \ldots, \mathrm{h}_{\mathrm{n}}^{\mathrm{T}}\right], \quad \mathrm{H} \in \mathbb{R}^{\mathrm{n} \times \mathrm{m}} \\
& \mathrm{K}^{\mathrm{T}}=\left[\mathrm{k}_{1}^{\mathrm{T}}, \mathrm{k}_{2}^{\mathrm{T}}, \ldots, \mathrm{k}_{\mathrm{n}}^{\mathrm{T}}\right], \quad \mathrm{K} \in \mathcal{R}^{\mathrm{n} \times \mathrm{m}}
\end{aligned}
$$

$\widehat{\mathrm{g}}(\widehat{\mathrm{z}}, \mathrm{u}, \mathrm{t}, w, \widehat{\theta})$ is the estimated function of the nonlinear/uncertain function $\mathrm{g}(\mathbf{z}, \mathbf{u}, \mathrm{t}, w, \theta)$. The estimated function $\widehat{\mathrm{g}}(\widehat{\mathbf{z}}, \mathbf{u}, \mathrm{t}, w, \hat{\boldsymbol{\theta}})$ is function of the estimated state, input, time, disturbance, and the estimated system parameter. The 
function $1_{s}(\widetilde{y})$ is the multivariable generalization of switching functions: $1_{\mathrm{s}}(\widetilde{\mathrm{y}})=\left[\operatorname{sgn}\left(\widetilde{\mathrm{y}}_{1}\right) \operatorname{sgn}\left(\widetilde{\mathrm{y}}_{2}\right) \ldots \operatorname{sgn}\left(\widetilde{\mathrm{y}}_{\mathrm{m}}\right)\right]^{\mathrm{T}}$. With this state estimator, the error dynamics is obtained by subtracting equation (2.3) from equation (2.1):

$$
\dot{\mathrm{x}}=(\mathrm{A}-\mathrm{H} \mathrm{C}) \mathrm{x}-\mathrm{K} \mathrm{I}_{\mathrm{s}}(\tilde{\mathrm{y}})+\left[\begin{array}{c}
0 \\
\vdots \\
0 \\
\mathrm{w}
\end{array} \mid\right.
$$

where $\mathrm{x}=\mathrm{z}-\widehat{\mathrm{z}}, \tilde{\mathrm{y}}=\mathrm{y}-\mathrm{C} \widehat{\mathrm{z}}=\mathrm{C} \mathrm{x}, \mathrm{w}=\mathrm{g}(\mathrm{z}, \mathrm{u}, \mathrm{t}, w, \boldsymbol{\theta})-\widehat{\mathrm{g}}(\widehat{\mathrm{z}}, \mathrm{u}, \mathrm{t}, w, \widehat{\theta}), \mathrm{C}=\left[\begin{array}{llll}1 & 0 & \ldots & 0\end{array}\right]$, the system matrix of error dynamics $A$ is $A=\left[\begin{array}{ll}0 & I \\ \hline 0 & 0\end{array}\right]$.

Since the function $\mathrm{g}(\mathrm{z}, \mathrm{u}, \mathrm{t}, w, \theta)$ can be a nonlinear function of the state, it is not modeled into the system matrix. For sliding observer error dynamics, $\mathrm{w}$ is the disturbance input which includes the system modeling errors, neglected nonlinearities, parametric uncertainties, and noises. Since the disturbance input $\mathrm{w}$ is the difference between $\mathrm{g}$ and $\hat{\mathrm{g}}$, its dynamics varies according to the modeling accuracy and the estimation error state [Slotine, 1987]. The estimated model complexity may depend on the acceptable computational burden. The bound of the disturbance input $w$ is assumed to be known for the proper range of system state.

This sliding observer is basically the conventional Luenberger-like observer with an additional term of the signum functions or saturation functions. The "error dynamics" of the observers will be studied in the light of this viewpoint.

Rewriting the equation (2.5), in the case of single measurement available, the observer error structure is of the following form:

$$
\left\{\begin{array}{l}
\dot{x}_{1}=x_{2}-h_{1} x_{1}-k_{1} \operatorname{sgn}\left(x_{1}\right) \\
\dot{x}_{2}=x_{3}-h_{2} x_{1}-k_{2} \operatorname{sgn}\left(x_{1}\right) \\
\cdots \\
\dot{x}_{n}=-h_{n} x_{1}-k_{n} \operatorname{sgn}\left(x_{1}\right)+w
\end{array}\right.
$$


Throughout this study, the above equation (2.6) is used as the standard form of the sliding observer error dynamics with a single measurement.

\subsection{Solutions of Discontinuous Differential Equations}

\subsubsection{Sliding and Passing Conditions}

According to the solution of discontinuous differential equations (see Appendix A.1), one should discern the conditions between the two main cases: one is the reaching dynamics and the other is sliding dynamics. Consider the case where the function $f(x)$ is discontinuous on a smooth surface $\mathrm{S}$ given by the equation $\mathrm{y}=\mathrm{C} \mathrm{x}=0$. The surface $\mathrm{S}$ separates its neighborhood in the state space into domains $\Omega$. and $\Omega_{+}$. For the point $\xi$ approaching the point $\mathrm{x} \in \mathrm{S}$ from the domains $\Omega_{-}$and $\Omega_{+}$, let the function $f(t, \xi)$ have the limit values: [Filippov, 1964]

$$
\lim _{\substack{\xi \in \Omega \\ \xi \rightarrow \mathrm{x}}} \mathrm{f}(\mathrm{t}, \xi)=\mathrm{f}(\mathrm{t}, \mathrm{x}), \lim _{\substack{\xi \in \Omega_{+} \\ \xi \rightarrow \mathrm{x}}} \mathrm{f}(\mathrm{t}, \xi)=\mathrm{f}^{+}(\mathrm{t}, \mathrm{x})
$$

The functions $f_{N}$ and $f_{N}^{+}$are defined as the normal component of the vectors $\mathrm{f}^{-}$and $\mathrm{f}^{4}$ to the surface $\mathrm{S}$ at the point $\mathrm{x}$. The positive sign is directed toward the domain $\Omega_{+}$. In this study, the velocity means $\dot{x}$ in (2.6).
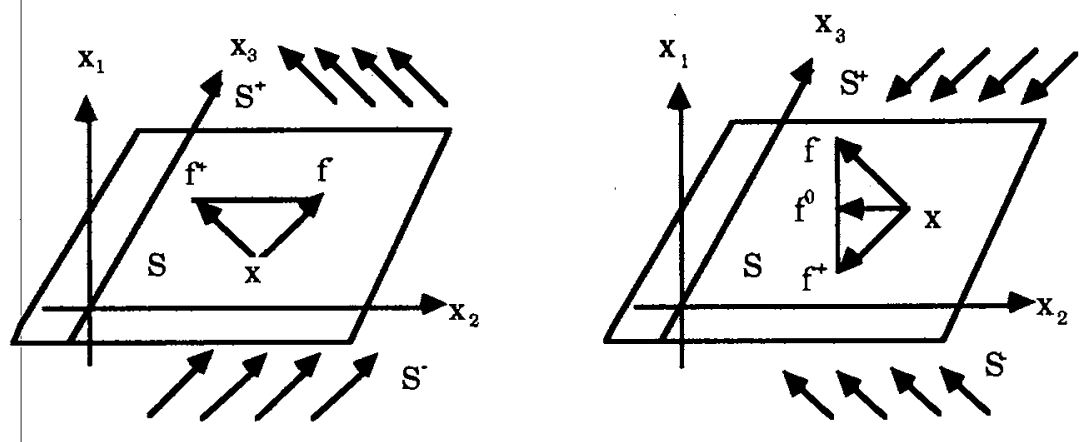

Figure 2.1 The velocity field near the hyperplane 
Sliding Condition. If the velocity field $\mathrm{f}(\mathrm{t}, \mathrm{x})$ is directed to the surface $\mathrm{S}$ on both sides, i.e., $\mathrm{f}_{\mathrm{N}}(t, \mathrm{x})>0, \mathrm{f}_{\mathrm{N}}^{+}(t, \mathrm{x})<0$, then near surface $\mathrm{S}$, all the solution points are approaching it from both sides as $t$ increases, and they cannot leave it while the condition is satisfied. Let the hyperplane equation be $\phi(x)=0$. For the sliding observer (2.6), $\phi(x)=x_{1}=0$ is the sliding surface, and the directional functions at the surface $\left(\mathrm{x}_{1}=0\right)$ are

$$
\left\{\begin{array}{l}
\mathrm{f}_{\mathrm{N}}=\frac{(\nabla \phi) . \mathrm{f}}{|\nabla \phi|}=\dot{\mathrm{x}}_{1 \cdot}=\mathrm{x}_{2}-\mathrm{h}_{1} \mathrm{x}_{1}+\mathrm{k}_{1}=\mathrm{x}_{2}+\mathrm{k}_{1} \\
\mathrm{f}_{\mathrm{N}}^{+}=\frac{(\nabla \phi) . \mathrm{f}^{+}}{|\nabla \phi|}=\dot{\mathrm{x}}_{1+}=\mathrm{x}_{2}-\mathrm{h}_{1} \mathrm{x}_{1}-\mathrm{k}_{1}=\mathrm{x}_{2}-\mathrm{k}_{1}
\end{array}\right.
$$

- The sliding condition for the system (2.6) is

$$
\mathbf{x}_{1} \cdot \dot{\mathbf{x}}_{1}<0\left(\text { at } \mathbf{x}_{1}=0\right)
$$

Plug equation (2.7) in equation (2.8):

$$
\left|\mathrm{x}_{2}\right|<\mathrm{k}_{1}
$$

Passing Condition. If the velocity field $f(t, x)$ has the same signs, i.e., $\mathrm{f}_{\mathrm{N}}(\mathrm{t}, \mathrm{x})<0 \quad \mathrm{f}_{\mathrm{N}}^{+}(\mathrm{t}, \mathrm{x})<0$ or $\mathrm{f}_{\mathrm{N}}(\mathrm{t}, \mathrm{x})>0 \quad \mathrm{f}_{\mathrm{N}}^{+}(\mathrm{t}, \mathrm{x})>0$, then near surface $\mathrm{S}$ all the solution points are passing through the hyperplane $\phi(x)=x_{1}=0$ as $t$ increases.

- The passing condition for the system (2.6) is

$$
\left|x_{2}\right|>k_{1}\left(\text { at } x_{1}=0\right)
$$

Suppose that for $\mathrm{t}^{\mathrm{i}}<\mathrm{t}<\mathrm{t}^{\mathrm{f}}$ the trajectory of vector function $\mathrm{x}(\mathrm{t})$ extends inside the region in which the right-hand side of equation (2.6) is continuous with respect to $(t, x)$. With this passing condition, the solution of this system is determined by the first case of the solution definition in Appendix A.1. Undoubtedly, if the velocity function $\dot{x}=f(t, x)$ is continuous at each half plane, then $x(t)$ is continuous. Consequently, the continuity of $x(t)$ is: 


$$
x(t)=x\left(t^{i}\right)+\int_{i^{i}}^{t} f(\tau, x(\tau)) d \tau
$$

where $\mathrm{x}^{\mathrm{i}}$ is the initial state at the passing point.

Since the integral is continuous, the continuous derivative $\dot{x}=f(t, x)$ exists, for each half domains, for all $t \in\left(t^{i}, t^{i}\right)$, i.e., $x(t)$ is a solution in the ordinary sense [Filippov, 1964] (see also Chapter 2.4.2).

\subsubsection{Switching System}

The suggested sliding observer is a linear system plus switching terms. The linear system has the linear correction term that corrects the velocity field proportional to the feedback state $x_{1}$. On the contrary, the switching terms change the velocity field according to the sign of feedback states. In a linear system, the state transition matrix is in an exponential form. The solution of the switching system is compared with the usual definition of the solution of the differential equation with a continuous right-hand side.

The first order n-simultaneous switching system is

$$
\left\{\begin{array}{c}
\dot{x}_{1}=x_{2}-k_{1} \operatorname{sgn}\left(x_{1}\right) \\
\dot{x}_{2}=x_{3}-k_{2} \operatorname{sgn}\left(x_{1}\right) \\
\vdots \\
\dot{x}_{n}=-k_{n} \operatorname{sgn}\left(x_{1}\right)+w
\end{array}\right.
$$

While the state $x_{1}$ is not zero (in other words the solution point leaves the sliding surface and does not cross over the hyperplane), these dynamics are known as "reaching dynamics." In the 2-order case, it is easy to verify the trajectories of the switching system using a phase plane analysis. Unfortunately, however, this technique does not apply directly to higher order systems. For the general case, the state is obtained by integrating the first order simultaneous equations. 


$$
\left\{\begin{aligned}
x_{n} & =-k_{n} \operatorname{sgn}\left(x_{1}\right) t+\int_{0}^{t} w d t+x_{n}^{i} \\
x_{n-1} & =-\frac{k_{n}}{2 !} \operatorname{sgn}\left(x_{1}\right) t^{2}+\iint_{0}^{t} w d t d t+\left(x_{n}^{i}-k_{n-1} \operatorname{sgn}\left(x_{1}\right)\right) t+x_{n-1}^{i} \\
& \vdots \\
x_{2} & =-\frac{k_{n}}{(n-1) !} \operatorname{sgn}\left(x_{1}\right) t^{n-1}+\iiint_{0}^{t} w d t^{n-1}+\frac{\left(x_{n}^{i}-k_{n-1} \operatorname{sgn}\left(x_{1}\right)\right)}{(n-2) !} t^{n-2}+\ldots+\left(x_{3}^{i}-k_{2} \operatorname{sgn}\left(x_{1}\right) t+x_{2}^{i}\right. \\
x_{1} & =-\frac{k_{n}}{n !} \operatorname{sgn}\left(x_{1}\right) t^{n}+\iiint_{0}^{t} w d t^{n}+\frac{\left(x_{n}^{i}-k_{n-1} \operatorname{sgn}\left(x_{1}\right)\right)}{(n-1) !} t^{n-1}+\ldots+\left(x_{2}^{i}-k_{1} \operatorname{sgn}\left(x_{1}\right)\right) t+x_{1}^{i}
\end{aligned}\right.
$$

where $x_{j}^{i}$ is the initial state of $x_{j}$ and $\iiint w d t^{n}$ stands for $n$-multiple integral.

Let the bound of disturbance be $w=w(t)$ as follows:

$$
-\mathrm{k}_{\mathrm{n}} \leq \mathrm{w}_{\min } \leq \mathrm{w} \leq \mathrm{w}_{\max } \leq \mathrm{k}_{\mathrm{n}}
$$

With the bounded initial state and disturbance, the bound of each state for the right half side of the switching plane $\left(x_{1}>0\right)$ is as follows:

$$
\begin{aligned}
& -k_{n} t+w_{\min } t+x_{n}^{i} \leq x_{n} \leq-k_{n} t+w_{\max } t+x_{n}^{i} \\
& -\frac{\left(\mathrm{k}_{\mathrm{n}}-\mathrm{w}_{\min }\right)}{2 !} \mathrm{t}^{2}-\left(\mathrm{k}_{\mathrm{n}-1}-\mathrm{x}_{\mathrm{n}}^{\mathrm{i}}\right) \mathrm{t}+\mathrm{x}_{\mathrm{n}-1}^{\mathrm{i}} \leq \mathrm{x}_{\mathrm{n}-1} \leq-\frac{\left(\mathrm{k}_{\mathrm{n}}-\mathrm{w}_{\max }\right)}{2 !} \mathrm{t}^{2}-\left(\mathrm{k}_{\mathrm{n}-1}-\mathrm{x}_{\mathrm{n}}^{\mathrm{i}}\right) \mathrm{t}+\mathrm{x}_{\mathrm{n}-1}^{\mathrm{i}} \\
& \frac{\left(k_{n}-w_{\min }\right)}{(n-1) !} t^{n-1}-\frac{\left(k_{n-1}-x_{n}^{i}\right)}{(n-2) !} t^{n-2}-\ldots-\left(k_{2}-x_{3_{0}}\right) t+x_{2}^{i} \leq x_{2} \\
& \leq-\frac{\left(k_{n}-w_{\max }\right)}{(n-1) !} t^{n-1}-\frac{\left(k_{n-1}-x_{n}^{i}\right)}{(n-2) !} t^{n-2}-\ldots-\left(k_{2}-x_{3_{0}}\right) t+x_{2}^{i} \\
& 0 \leq-\frac{\left(k_{n}-w_{\min }\right)}{n !} t^{n}-\frac{\left(k_{n-1}-x_{n_{0}}\right)}{(n-1) !} t^{n-1} \quad \ldots\left(k_{1}-x_{2_{0}}\right) t+x_{1_{0}} \leq x_{1} \\
& \leq-\frac{\left(k_{n}-w_{\max }\right)}{n !} t^{n}-\frac{\left(k_{n-1}-x_{n_{o}}\right)}{(n-1) !} t^{n-1}-\ldots\left(k_{1}-x_{2_{0}}\right) t+x_{1_{0}}
\end{aligned}
$$


For the left half plane $\left(x_{1}<0\right)$, the bound will be obtained the same way. We cannot conclude the stability of the system from the equations (2.15). However, the equations show that each state has modes of power of time. Particularly, the coefficient of the highest power term of each state has the opposite sign of $\mathbf{x}_{1}$.

\subsection{Sliding Observer Dynamics}

Since the sliding observer has both linear correction terms and switching terms, its dynamics may have characteristics of both. In this section, the sliding observer is compared with a linear system and a switching system and the sliding observer dynamics are reexamined as a composition of reaching dynamics and sliding dynamics.

\subsubsection{Reaching dynamics}

Linear Time Invariant (LTI) System. A LTI system in state space form is

$$
\dot{z}=A z+B u, \quad z\left(t_{0}\right)=z_{0}
$$

The state solution is expressed in an exponential form of the statetransition matrix:

$$
z(t)=e^{A t} z_{o}+\int_{0}^{t} e^{A(t-\tau)} B u(\tau) d \tau
$$

The responses of a linear time-invariant dynamical system are dictated mainly by its modes, or equivalently, the eigenvalues of $A$. If an eigenvalue has a negative real part, its mode will approach zero exponentially as $t \rightarrow \infty$.

$$
z(t)=\sum_{i}\left(p_{i} z_{0}\right) e^{\lambda_{i} t} q_{i}
$$

where $p_{i}$ and $q_{i}$ are, respectively, a right and a left eigenvectors of A associated 
with $\lambda_{\mathrm{i}}$

Switching System. The switching dynamics (2.15) is compared to the time invariant linear system dynamics. The state correction mechanism is compared to the LTI system as follows:

Switching system:

$$
\left\{\begin{array}{l}
\mathrm{x}_{2}=\dot{\mathrm{x}}_{1}+\mathrm{k}_{1} \operatorname{sgn}\left(\mathrm{x}_{1}\right) \\
\mathrm{x}_{3}=\dot{\mathrm{x}}_{2}+\mathrm{k}_{2} \operatorname{sgn}\left(\mathrm{x}_{1}\right) \\
\cdots \\
\mathrm{x}_{\mathrm{n}}=\dot{\mathrm{x}}_{\mathrm{n}-1}+\mathrm{k}_{\mathrm{n}-1} \operatorname{sgn}\left(\mathrm{x}_{1}\right)
\end{array}\right.
$$

LTI system:

$$
\left\{\begin{array}{c}
\mathrm{x}_{2}=\dot{\mathrm{x}}_{1}+\mathrm{h}_{1} \mathrm{x}_{1} \\
\mathrm{x}_{3}=\dot{\mathrm{x}}_{2}+\mathrm{h}_{2} \mathrm{x}_{1} \\
\cdots \\
\mathrm{x}_{\mathrm{n}}=\dot{\mathrm{x}}_{\mathrm{n}-1}+\mathrm{h}_{\mathrm{n}-1} \mathrm{x}_{1}
\end{array}\right.
$$

Reaching Dynamics of the Sliding Observer. The sliding observer error dynamics is the same as the combination of two systems. Consider a 3-order case of the sliding observer with a passing condition:

$$
\left\{\begin{array}{l}
\dot{x}_{1}=x_{2}-h_{1} x_{1}-k_{1} \operatorname{sgn}\left(x_{1}\right) \\
\dot{x}_{2}=x_{3}-h_{2} x_{1}-k_{2} \operatorname{sgn}\left(x_{1}\right) \\
\dot{x}_{3}=-h_{3} x_{1}-k_{3} \operatorname{sgn}\left(x_{1}\right)+w
\end{array}\right.
$$

where $\mathrm{w}=\omega(\mathrm{x}, \mathrm{u}, \mathrm{t}, \mathrm{w}, \theta)$

Differentiate $\dot{\mathbf{x}}_{1}$ and plug in the second equation:

$$
\ddot{x}_{1}=-h_{1} \dot{x}_{1}-h_{2} x_{1}-k_{1} \delta\left(x_{1}\right) \dot{x}_{1}-k_{2} \operatorname{sgn}\left(x_{1}\right)+x_{3}
$$

where $\delta\left(x_{1}\right)$ is a dirac delta function (see Appendix A.2)

$$
\mathrm{x}_{1}^{(3)}=-\mathrm{h}_{1} \ddot{\mathrm{x}}_{1}-\mathrm{h}_{2} \dot{\mathrm{x}}_{1}-\mathrm{h}_{3} \mathrm{x}_{1}-\mathrm{k}_{1} \delta\left(\mathrm{x}_{1}\right) \ddot{\mathrm{x}}_{1}-\mathrm{k}_{2} \delta\left(\mathrm{x}_{1}\right) \dot{\mathrm{x}}_{1}-\mathrm{k}_{3} \operatorname{sgn}\left(\mathrm{x}_{1}\right)+\mathrm{w}
$$


If a solution point does not cross over the hyperplane, i.e., reaching dynamics and $x_{1}$ is not zero, then the dynamics (2.22) is rewritten as:

$$
x_{1}^{(3)}=-h_{1} \ddot{x}_{1}-h_{2} \dot{x}_{1}-h_{3} x_{1}-k_{3} \operatorname{sgn}\left(x_{1}\right)+w
$$

Near the switching plane, i.e., $\left|x_{1}\right| \ll 1$, the contribution of linear correction terms in the equation (2.21) is less than that of switching terms. However, the linear correction terms of the reaching dynamics cannot be neglected. Even though $\left|x_{1}\right| \ll 1$, we should notice that the differentiated terms may not be small. Hence, by using the linear correction terms, we can stabilize the system.

\subsubsection{Sliding Dynamics}

To get the sliding dynamics, the methodology of Lemma 1.1 in Appendix A. 1 could be directly applied to the sliding observer[Filippov, 1964]. With a finite switching timing, the sliding dynamics includes chattering that may be harmful for sliding control in practice. For an observer problem, even though the chattering is only a numeric phenomenon, it is not desirable for the observer based controllers. With the linear correction terms, the sliding dynamics is obtained as the same method.

The $n-1$ poles associated with the sliding dynamics on the sliding patch are obtained by Slotine [1987]

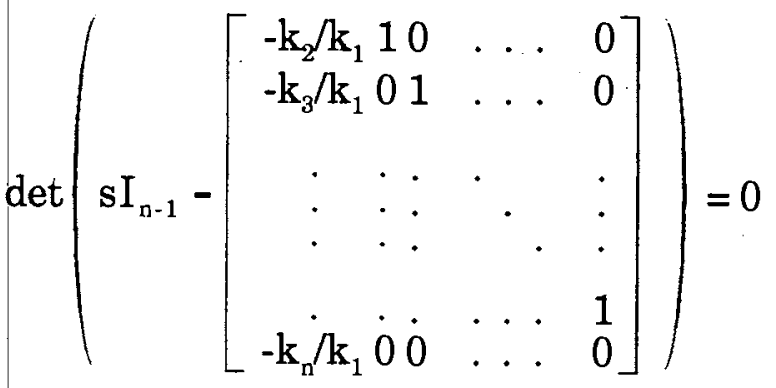


where the $I_{n-1}$ is the identity matrix of order $n-1$. Thus the poles of the sliding dynamics can be placed arbitrarily by proper selection of the ratios $\mathrm{k}_{\mathrm{j}} / \mathrm{k}_{1},(\mathrm{i}=2, \ldots, \mathrm{n})$.

\subsection{Coordinate Transformation}

\subsubsection{Shifted-coordinate System}

The error dynamics (2.6) can be rewritten as:

$$
\dot{x}=A_{m} x-K \operatorname{sgn}\left(x_{1}\right)+W
$$

where $\mathrm{K}=\left\{\mathrm{k}_{1}, \ldots, \mathrm{k}_{\mathrm{n}}\right\}^{\mathrm{T}}, \mathrm{W}=\{0, \ldots, 0 \mathrm{w}\}^{\mathrm{T}}$ and

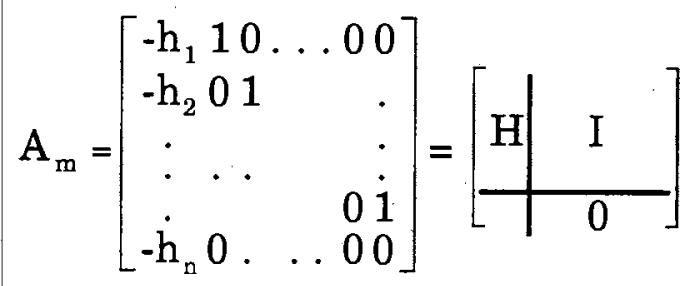

The equation (2.25) can be rewritten as:

$$
\dot{x}=A_{m} x-\left[\begin{array}{c|c}
I & 0 \\
\hline 0 & 0
\end{array}\right] K \operatorname{sgn}\left(x_{1}\right)+\left[\begin{array}{c}
0 \\
\vdots \\
0 \\
u_{d}
\end{array}\right)
$$

where $u_{d}=w-k_{n} \operatorname{sgn}\left(x_{1}\right)$

Observation 2.2 Matrix multiplication

$$
\left[\begin{array}{l|l}
\mathrm{H} \mid \\
\mathrm{I} \\
\hline 0
\end{array}\right]\left[\begin{array}{l|l}
0 & 0 \\
\hline \mathrm{I} & 0
\end{array}\right]=\left[\begin{array}{l|l}
\mathrm{I} & 0 \\
\hline 0 & 0
\end{array}\right]
$$


By using equation (2.29), rewrite equation (2.27):

$$
\dot{x}=A_{m}\left(x-\left[\begin{array}{c|c}
0 & 0 \\
\hline I & 0
\end{array}\right] K \operatorname{sgn}\left(x_{1}\right)\right)+\left(\begin{array}{c}
0 \\
\vdots \\
0 \\
u_{d}
\end{array}\right)
$$

The sliding observer can be rewritten:

$$
\dot{\mathrm{x}}=\mathrm{A}_{\mathrm{m}}\left(\mathrm{x}-\mathrm{K}_{\mathrm{s}} \operatorname{sgn}\left(\mathrm{x}_{1}\right)\right)+\left\{\begin{array}{c}
0 \\
\vdots \\
0 \\
\mathrm{u}_{\mathrm{d}}
\end{array}\right\}
$$

$$
\text { where } \quad \mathrm{K}_{\mathrm{s}}=\left[\begin{array}{c|c}
0 & 0 \\
\hline \mathrm{I} & 0
\end{array}\right] \mathrm{K} \quad: \text { shifted switching coefficient }
$$

Take a coordinate transformation:

$$
\mathrm{x}_{\mathrm{s}}=\mathrm{x}-\mathrm{K}_{\mathrm{s}} \operatorname{sgn}\left(\mathrm{x}_{1}\right)
$$

and differentiate it

$$
\dot{\mathrm{x}}_{\mathrm{s}}=\dot{\mathrm{x}}-\mathrm{K}_{\mathrm{s}} \delta(\mathrm{x}) \dot{\mathrm{x}}_{1}
$$

Finally, in the shifted-coordinate system, the sliding observer is

$$
\dot{x}_{s}=A_{m} x_{s}+\left\{\begin{array}{c}
0 \\
\vdots \\
0 \\
u_{d}
\end{array}\right\}-K_{s} \delta(x) \dot{x}_{1}
$$

For the reaching dynamics, the sliding observer in the shifted-coordinate is

$$
\dot{\mathrm{x}}_{\mathrm{s}}=\mathrm{A}_{\mathrm{m}} \mathrm{x}_{\mathrm{s}}+\left\{\begin{array}{c}
0 \\
\vdots \\
0 \\
u_{\mathrm{d}}
\end{array}\right\}
$$


where $u_{d}=w-k_{n} \operatorname{sgn}\left(x_{1}\right)$

The shifted-coordinate transformation for each side of the domains $\Omega_{+}$ and $\Omega$. are as follows:

$$
x_{s}=\left\{\begin{array}{l}
x_{+}=x-K_{s}, \text { for } \Omega_{+}\left(x_{1}>0\right) \\
x_{-}=x+K_{s}, \text { for } \Omega_{-}\left(x_{1}<0\right)
\end{array}\right.
$$

\subsubsection{Sliding Observer Error Dynamics in the shifted coordinate}

Compared to a LTI system, the sliding observer has three dynamics which are two reaching dynamics for $\mathrm{x}_{1}>0, \mathrm{x}_{1}<0$ in the shifted-coordinate and sliding dynamics at the hyperplane $x_{1}=0$.

i) Right Reaching Dynamics. The first mode is for $x_{1}>0$ space with passing condition:

$$
\left\{\begin{array}{l}
\dot{x}_{1}=x_{2}-h_{1} x_{1}-k_{1} \\
\dot{x}_{2}=x_{3}-h_{2} x_{1}-k_{2} \\
\cdots \\
\dot{x}_{n}=w-h_{n} x_{1}-k_{n}
\end{array}\right.
$$

Take the coordinate transformation:

$$
\left\{\begin{array}{l}
x_{1+}=x_{1} \\
x_{2+}=x_{2}-k_{1} \operatorname{sgn}\left(x_{1}\right) \\
\cdots \\
x_{n+}=x_{n}-k_{n-1} \operatorname{sgn}\left(x_{1}\right)
\end{array}\right.
$$

The right reaching dynamics is

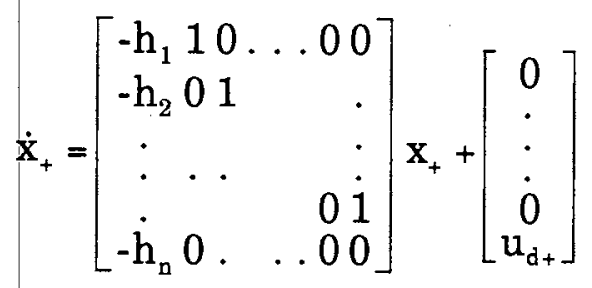

where $\mathrm{x}_{+}=\left[\mathrm{x}_{1+} \ldots \mathrm{x}_{\mathrm{n}+}\right]^{\mathrm{T}}$, for $\mathrm{x}_{1} \neq 0, \delta\left(\mathrm{x}_{1}\right)=0$ and $\mathrm{u}_{\mathrm{d}+}=-\mathrm{k}_{\mathrm{n}}+\mathrm{w}<0$ for $\mathrm{k}_{\mathrm{n}}>|\mathrm{w}|_{\max }$

The reaching dynamics is the second companion form in the linear 
system theory. The equilibrium point is moved to:

$$
\mathrm{x}=\left[0 \mathrm{k}_{1} \mathrm{k}_{2} \ldots \mathrm{k}_{\mathrm{n}-1}\right]^{\mathrm{T}}
$$

The disturbance input $u_{d+}$ will push the solution point into the sliding patch where the sliding mode occurs or the trajectory will cross the hyperplane.

ii) Left Reaching Dynamics. The second mode is for $\mathbf{x}_{1}<0$ space with passing condition:

$$
\left\{\begin{array}{c}
\dot{x}_{1}=x_{2}-h_{1} x_{1}+k_{1} \\
\dot{x}_{2}=x_{3}-h_{2} x_{1}+k_{2} \\
\cdots \\
\dot{x}_{n}=w-h_{n} x_{1}+k_{n}
\end{array}\right.
$$

The shifted-coordinate transformation is

$$
\left\{\begin{array}{l}
\mathrm{x}_{1-}=\mathrm{x}_{1} \\
\mathrm{x}_{2-}=\mathrm{x}_{2}+\mathrm{k}_{1} \\
\cdots \\
\mathrm{x}_{\mathrm{n}-}=\mathrm{x}_{\mathrm{n}}+\mathrm{k}_{\mathrm{n}-1}
\end{array}\right.
$$

The left reaching dynamics is

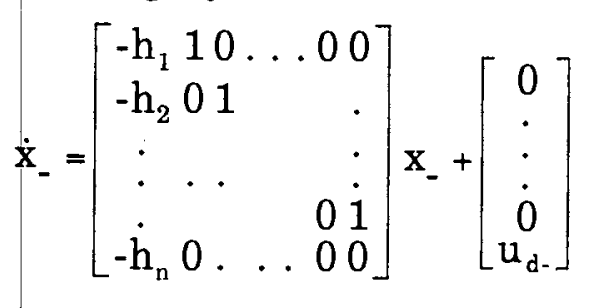

where $x_{-}=\left[x_{1} \ldots x_{n--}\right]^{T}$ and $u_{d_{-}}=k_{n}+w>0$

The equilibrium point is moved to $x=\left[0-k_{1}-k_{2} \ldots-k_{n-1}\right]^{T}$.

The disturbance input $u_{d}$. will push the solution point into the sliding zone where the sliding mode occurs or the trajectory will cross the hyperplane. iii) Sliding Dynamics. The third dynamics is the sliding dynamics that 
is in the hyperplane $x_{1}=0$ with the sliding condition $\left(\left|x_{2}\right|<k_{1}\right)$.

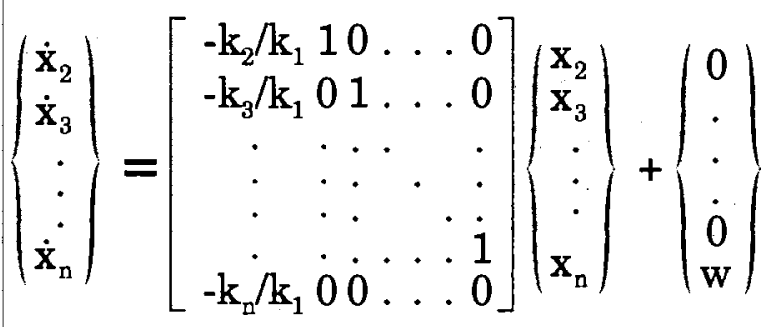

The sliding patch is the region:

$$
\mathrm{x}_{1}=0,-\mathrm{k}_{1}<\mathrm{x}_{2}<\mathrm{k}_{1}, \ldots,-\mathrm{k}_{\mathrm{n}-1}<\mathrm{x}_{\mathrm{n}}<\mathrm{k}_{\mathrm{n}-1} \text {. }
$$

A sliding patch is defined as a zone where sliding condition is satisfied and where the sign of velocities are the same. For the sliding observer, the sliding zone is

$$
-\mathrm{k}_{1}<\mathrm{x}_{2}<\mathrm{k}_{1}
$$

In the shifted-coordinate, it is equivalent to:

$$
\begin{aligned}
& -2 \mathrm{k}_{1}<\mathrm{x}_{2+}<0 \\
& 0<\mathrm{x}_{2 .}<2 \mathrm{k}_{1}
\end{aligned}
$$

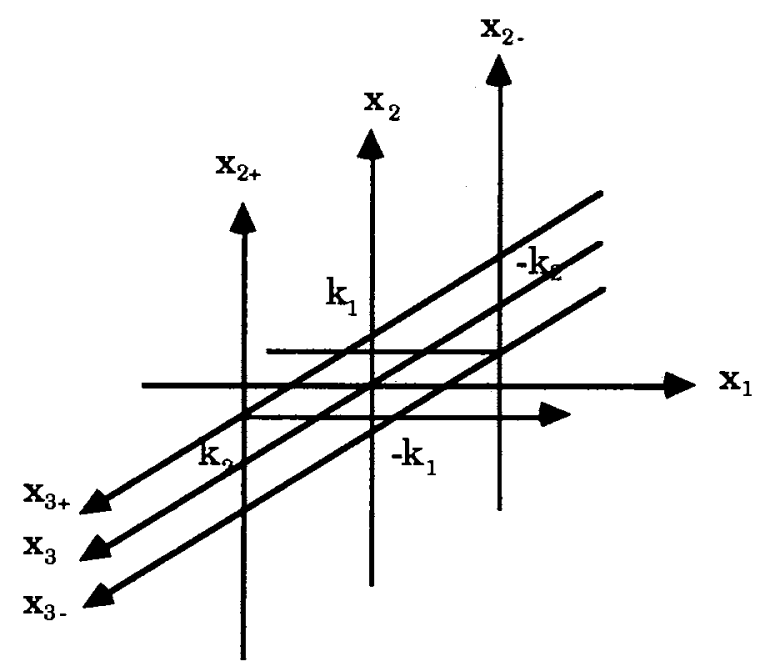

Figure 2.2 Shifted-coordinate and sliding patch 
Steady State of the Sliding Dynamics. With the constant disturbance, the steady state with the sliding condition can be calculated from the condition:

$$
\left\{\begin{array}{c}
\dot{x}_{1}=0 \\
\dot{x}_{2}=0 \\
\cdots \\
\dot{x}_{n}=0
\end{array}\right.
$$

Plug this condition into the sliding observer (2.6) with $\mathrm{x}_{1}=0$ and obtain:

$$
\text { Time mean }\left[\operatorname{sgn}\left(\mathrm{x}_{1}\right)\right]=\frac{\mathrm{x}_{2}}{\mathrm{k}_{1}}=\frac{\mathrm{x}_{3}}{\mathrm{k}_{2}}=\ldots=\frac{\mathrm{w}}{\mathrm{k}_{\mathrm{n}}}
$$

From this condition, the steady states are obtained as follows:

$$
\left\{\begin{array}{c}
\mathrm{x}_{2 \mathrm{ss}}=\frac{\mathrm{k}_{1}}{\mathrm{k}_{\mathrm{n}}} \mathrm{w} \\
\mathrm{x}_{3 \mathrm{ss}}=\frac{\mathrm{k}_{2}}{\mathrm{k}_{\mathrm{n}}} \mathrm{w} \\
\mathrm{x}_{\mathrm{nss}}=\frac{\ddot{\mathrm{k}}_{\mathrm{n}-1}}{\mathrm{k}_{\mathrm{d}}} \mathrm{w}
\end{array}\right.
$$

With the presumed condition $|w| \leq k_{n}$, the steady state is in the region $\left|\mathrm{x}_{\mathrm{iss}}\right| \leq \mathrm{k}_{\mathrm{i}-1}$; in other words the steady state is confined by the shifted-constant $\mathrm{K}_{\mathrm{s}}$. If the absolute of every state is less than the shifted constant, i.e., $\left|\mathbf{x}_{\mathrm{i}}\right| \leq \mathrm{k}_{\mathrm{i}-1}$, then the switching terms dominate the velocity field direction at the each switching instance. Consequently, all the velocity direction is opposite of the sign of $x_{1}$ at the instance.

\subsubsection{Passing Points on the Hyperplane}

The error state of sliding observer with an arbitrary initial condition is desirable to converged to, within finite time of passing, the sliding patch and stays on it. Through a series of switching, designing an asymptotically 
stable system is required the notion of passing points. As a notational convenience, the set of passing points is defined by sequences of switching timing $0 \leq \tau_{j}<\infty, \forall \tau_{j} \in \tau_{s}=\tau_{0}, \tau_{1}, \ldots, \tau_{k-1}, \tau_{k}, \ldots$

$$
\left\{\mathrm{s}\left(\mathrm{x}_{\mathrm{s}}, \tau_{\mathrm{j}}\right)\right\}=\mathrm{s}\left(\tau_{0}\right), \mathrm{s}\left(\tau_{1}\right), \ldots, \mathrm{s}\left(\tau_{\mathrm{k}}\right), \ldots
$$

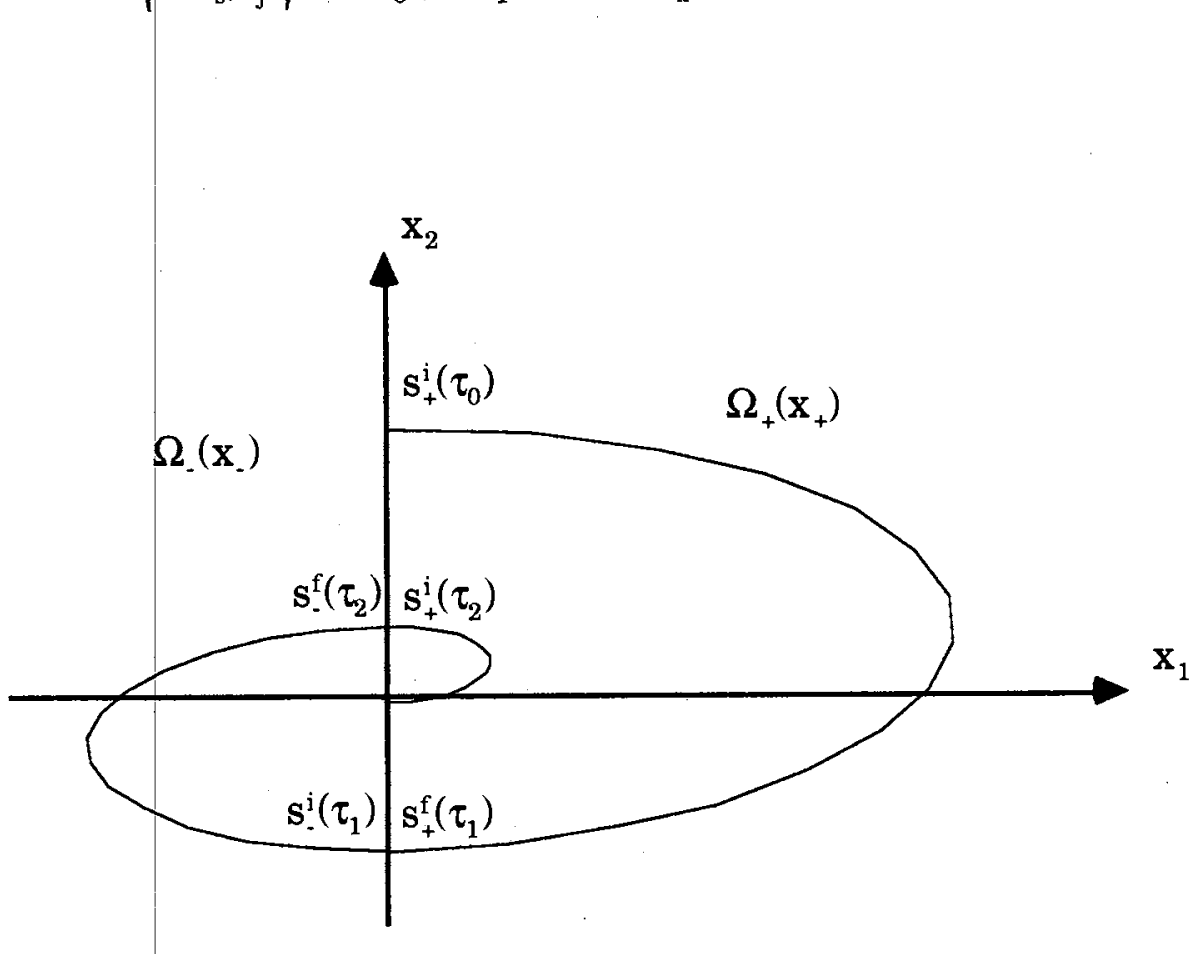

Figure 2.3 Passing points on the hyperplane

At the passing instance $\tau_{\mathrm{j}}$ on the hyperplane, the new initial state is reset as the same as the final state of the former reaching dynamics except the changed sign of $x_{1}\left(x_{1} \approx 0\right)$. Even though the velocity field of the system is discontinuous as equation (2.42) and (2.46), the trajectory is continuous (see Appendix A.1). The pasing point $s\left(x_{s}, \tau_{j}\right)$ is decomposed into $s_{+}\left(\tau_{j}\right)$ and $s_{-}\left(\tau_{j}\right)$ according to the sign of $x_{1}$. Hence, the continuity equation of passing state is

$$
\left\{\begin{array}{l}
s_{s}\left(\tau_{j}\right)=s_{(}^{i}\left(\tau_{j}\right)=s_{+}^{f}\left(\tau_{j}\right) \\
s_{s}\left(\tau_{j}\right)=s_{+}^{i}\left(\tau_{j}\right)=s_{-}^{f}\left(\tau_{j}\right)
\end{array} \quad \text { except changed sign of } x_{1}, s=-,+\right)
$$


where $s_{+}^{i}\left(\tau_{j}\right)=\left[\begin{array}{c}0_{+}^{i} \\ s_{2+}^{i} \\ \vdots \\ s_{n+}^{i}\end{array}\right], s_{+}^{f}\left(\tau_{j}\right)=\left[\begin{array}{c}0_{+}^{f} \\ s_{2+}^{f} \\ \vdots \\ s_{n+}^{f}\end{array}\right], s_{-}^{i}\left(\tau_{j}\right)=\left[\begin{array}{c}0_{-}^{i} \\ s_{2-}^{i} \\ \vdots \\ s_{n--}^{i}\end{array}\right], s_{-}^{f}\left(\tau_{j}\right)=\left[\begin{array}{c}f \\ 0_{-}^{f} \\ s_{2-}^{f} \\ \vdots \\ s_{n-}^{f}\end{array}\right]$

It is convenient to express the initial state and the final state in the shifted-coordinate $\mathrm{x}$

$$
\sigma_{+}^{i}\left(\tau_{j}\right)=\left[\begin{array}{c}
0_{+}^{i} \\
\sigma_{2+}^{i} \\
\vdots \\
\sigma_{n+}^{i}
\end{array}\right], \sigma_{+}^{f}\left(\tau_{j}\right)=\left[\begin{array}{c}
f \\
0_{+} \\
\sigma_{2+}^{f} \\
\vdots \\
\sigma_{n+}^{f}
\end{array}\right], \sigma_{-}^{i}\left(\tau_{j}\right)=\left[\begin{array}{c}
0_{-}^{i} \\
\sigma_{2-}^{i} \\
\vdots \\
\sigma_{n-}^{i}
\end{array}\right], \sigma_{-}^{f}\left(\tau_{j}\right)=\left[\begin{array}{c}
f \\
0 \\
\sigma_{2-}^{f} \\
\vdots \\
\sigma_{n-}^{f}
\end{array}\right]
$$

The relations of the passing state between the original coordinate and the shifted-coordinate are

$$
\left\{\begin{array}{l}
\sigma_{+}^{i}\left(\tau_{j}\right)=s_{+}^{i}\left(\tau_{j}\right)-K_{s} \\
\sigma_{+}^{f}\left(\tau_{j}\right)=s_{+}^{f}\left(\tau_{j}\right)-K_{s} \\
\sigma_{-}^{i}\left(\tau_{j}\right)=s_{-}^{i}\left(\tau_{j}\right)+K_{s} \\
\sigma_{-}^{f}\left(\tau_{j}\right)=s_{-}^{f}\left(\tau_{j}\right)+K_{s}
\end{array}\right.
$$

By plugging equation (2.59) into the continuity equation (2.53), we have:

i) Passing left (with the passing condition $x_{1}=0, x_{2}<-k_{1}$ )

$$
s_{-}^{i}\left(\tau_{j}\right)=s_{+}^{f}\left(\tau_{j}\right) \Rightarrow \sigma_{.}^{j}\left(\tau_{j}\right)=\sigma_{+}^{f}\left(\tau_{j}\right)+2 K_{s}
$$

ii) Passing right (with the passing condition $\mathrm{x}_{1}=0, \mathrm{x}_{2}>\mathrm{k}_{1}$ )

$$
s_{+}^{i}\left(\tau_{j}\right)=s_{-}^{f}\left(\tau_{j}\right) \Rightarrow \sigma_{+}^{j}\left(\tau_{j}\right)=\sigma_{-}^{f}\left(\tau_{j}\right)-2 K_{s}
$$

In the shifted-coordinate, the initial and final states are different from each other by twice of the shifted-coefficient because the coordinate origin is shifted. 


\section{CHAPTER III}

\section{STABILITY ANALYSIS}

\subsection{Worst Case Analysis}

\subsubsection{Worst Case Analysis of Switching System}

The initial condition and the disturbance input of the sliding observer determine a trajectory of system. For the sliding observer, the disturbance input can worsen the error state. If the worst direction is known and the worst case is stable, then the system is obviously stable. The worst-case trajectory is defined as the worst-convergence trajectory that encloses any other trajectory with the same bounded disturbance in the phase plane.

Consider the worst case in a second-order switching system with a passing condition.

$$
\left\{\begin{array}{l}
\dot{x}_{1}=x_{2}-k_{1} \operatorname{sgn}\left(x_{1}\right) \\
\dot{x}_{2}=-k_{2} \operatorname{sgn}\left(x_{1}\right)+w
\end{array}\right.
$$

where $|w|<k_{2}$ is a disturbance whose bound is known

With no loss of generality, we can assume that an initial point starts on a hyperplane, i.e., $x_{1}=0,\left|x_{2}\right|>k_{1}$. In a second-order phase plane analysis, the plane is divided into 4 regions as Figure 3.1.

Region I) For the region $\left(x_{1}>0 \cap x_{2}>k_{1}\right)$, the system is

$$
\left\{\begin{array}{l}
\dot{\mathrm{x}}_{1}=\mathrm{x}_{2}-\mathrm{k}_{1} \\
\dot{\mathrm{x}}_{2}=-\mathrm{k}_{2}+\mathrm{w}
\end{array}\right.
$$


The line ABC, in Figure 3.1, is the worst trajectory of solution point by the maximum disturbance $w=\omega$ and the trajectory equation is

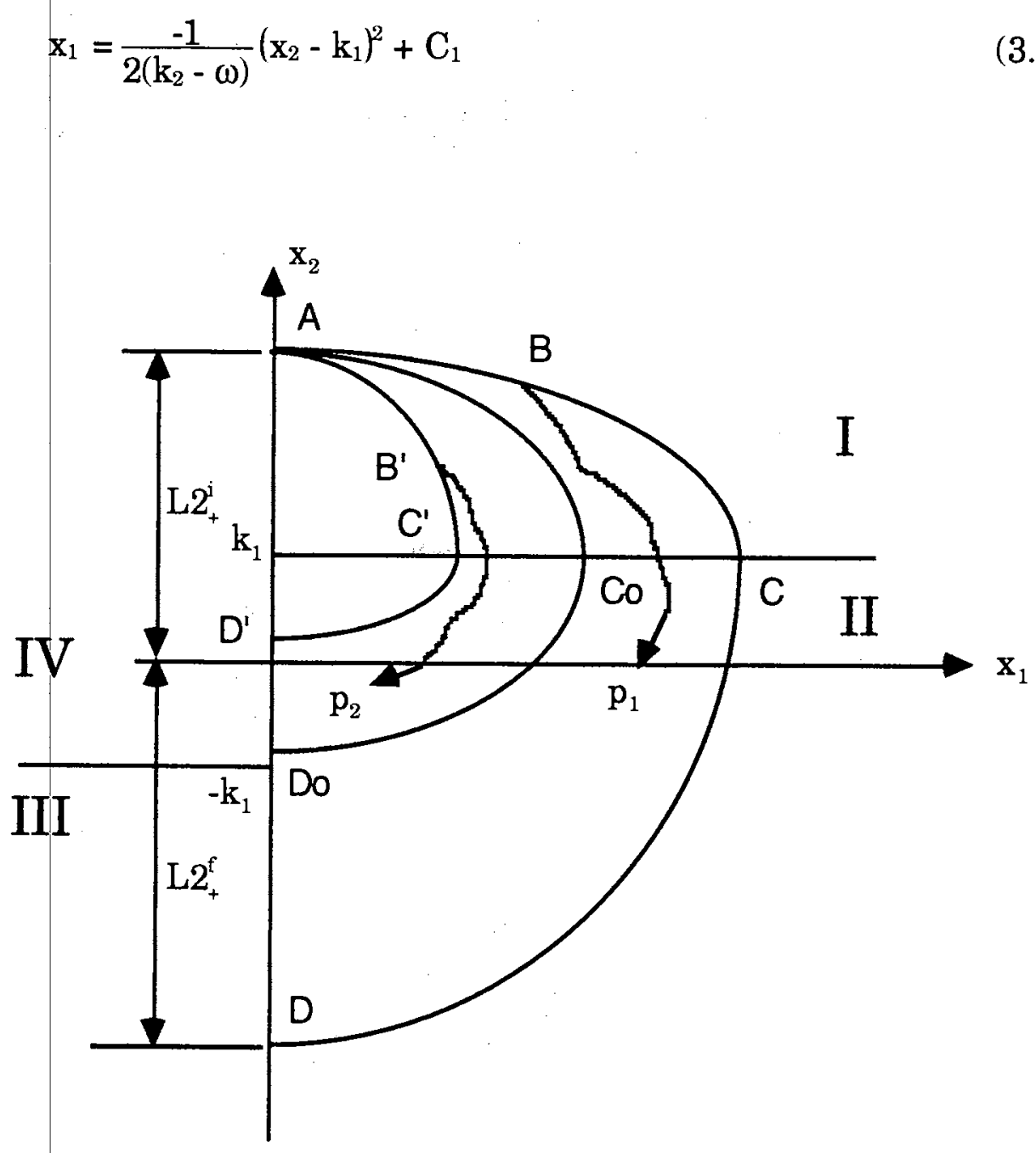

Figure 3.1 Worst case analysis in the second-order phase plane

As the disturbance input $w$ gets bigger, the arc of trajectory shows a wider angle. The worst point at $\mathrm{x}_{2}=\mathrm{k}_{1}$ by the above parabolic equation (3.4) is C. If the disturbance has negative sign, then it will be helpful to converge to the sliding patch. The trajectory equation is

$$
\mathrm{x}_{1}=\frac{-1}{2\left(\mathrm{k}_{2}+\omega\right)}\left(\mathrm{x}_{2}-\mathrm{k}_{1}\right)^{2}+\mathrm{C}_{1}
$$


Region II) For the region ( $\mathrm{x}_{1}>0 \cap \mathrm{x}_{2}<\mathrm{k}_{1}$ ), the system is the same as the case of region I. The worst trajectory of solution point is the line CD in Figure, that is by minimum disturbance $w=-\omega$ and the equation is

$$
\mathrm{x}_{1}=\frac{-1}{2\left(\mathrm{k}_{2}+\omega\right)}\left(\mathrm{x}_{2}-\mathrm{k}_{1}\right)^{2}+\mathrm{C}_{2}
$$

The final solution point $\mathrm{D}$ in this region is clearly the worst point because it is the outermost point. If the disturbance has a positive sign, then it will be helpful to converge like the trajectory line $C$ ' $D$ ' whose equation is

$$
x_{1}=\frac{-1}{2\left(k_{2}-\omega\right)}\left(x_{2}-k_{1}\right)^{2}+C_{2}^{\prime}
$$

If the bound of disturbance is known and if the coefficient $k_{2}$ is greater than the bound of disturbance, then the trajectories of solution point are enveloped with the curves, i.e., the worst trajectory $\mathrm{ABCD}$ and the best trajectory $\mathrm{AB}$ ' $\mathrm{C}$ ' $\mathrm{D}$ ' as in Figure. According to the equations (3.4) and (3.6), the worst direction of disturbance in right half plane is

$$
\begin{aligned}
\mathrm{w} & =\omega \operatorname{sgn}\left(\mathrm{x}_{2}-\mathrm{k}_{1} \operatorname{sgn}\left(\mathrm{x}_{1}\right)\right) \\
& =\omega \operatorname{sgn}\left(\dot{\mathrm{x}}_{1}\right)
\end{aligned}
$$

For a left half plane, the worst direction is the same as the above equation, since it is symmetric about the origin. Let assume the initial states $x_{1}^{i}=0$ and $x_{2}^{i}=L 2_{+}^{i}>k_{1}$. For the worst case, the constants of equations (3.4) and (3.6) are:

$$
\mathrm{C}_{1}=\mathrm{C}_{2}=\frac{\left(\mathrm{L} 2_{+}^{\mathrm{i}}-\mathrm{k}_{1}\right)^{2}}{2\left(\mathrm{k}_{2}-\omega\right)}
$$

With the given condition (3.9), solve the equation (3.6), and the final point, $\mathbf{x}_{2 \mid \mathbf{x}_{1}=0}$, is obtained for the worst case: 


$$
\begin{aligned}
& \mathrm{x}_{2 \mathrm{x}_{1}=0}=-\sqrt{\left(\mathrm{k}_{2}+\omega\right) \frac{\left(\mathrm{L} 2_{+}^{\mathrm{i}}-\mathrm{k}_{1}\right)^{2}}{\left(\mathrm{k}_{2}-\omega\right)}}+\mathrm{k}_{1} \\
& \mathrm{~L} 2_{-}^{\mathrm{f}}=\left|\mathrm{x}_{2 \mid \mathrm{x}_{1}=0}\right| \\
& \quad=\sqrt{\frac{\left(\mathrm{k}_{2}+\omega\right)}{\left(\mathrm{k}_{2}-\omega\right)}\left(\mathrm{L} 2_{+}^{\mathrm{i}}-\mathrm{k}_{1}\right)-\mathrm{k}_{1} \quad \text { (Take positive term) }}
\end{aligned}
$$

If $\mathrm{L} 2_{+}^{\mathrm{i}} \geq \mathrm{L} 2_{-}^{f}$, then the system is $\mathrm{BIBO}$ stable. If the disturbance is zero $(\omega=0)$, then the distance of the final point is closer than the initial condition by twice of $k_{1}$ until it reaches sliding patches. The worst case analysis of the left half plane is the same as that of right half plane. This jump approach to origin shows "shearing effect" of the sliding observer.

$$
\begin{aligned}
& \mathrm{L} 2_{+}^{\mathrm{f}}=\left(\mathrm{L} 2_{+}^{\mathrm{i}}-2 \mathrm{k}_{1}\right) \\
& \mathrm{L} 2_{-}^{\mathrm{f}}=\left(\mathrm{L} 2_{-}^{\mathrm{i}}-2 \mathrm{k}_{1}\right)
\end{aligned}
$$

With the known bound of $\omega$ and $L 2_{+}^{i}$ (or $L 2_{-}^{i}$ ), we need to design $k_{1}$ and $k_{2}$. ( $\mathrm{k}_{2}$ is presumed greater than the bound of $\omega$ ).

The algebraic condition for $k_{1}$ is

$$
\frac{\mathrm{k}_{1}}{\mathrm{~L} 2_{+}^{\mathrm{i}}} \geq\left(\frac{\mathrm{k}_{2}}{\omega}-\sqrt{\left(\frac{\mathrm{k}_{2}}{\omega}\right)^{2}-1}\right)
$$

The above equation is singular for $\omega=0$ : since this analysis is for the worse case, it is reasonable to assume $\omega \neq 0$. From the algebraic stability criterion, if $\mathrm{k}_{2}$ equals $\omega$ then the initial condition $\mathrm{x}_{2}$ should be on the sliding patch i.e., $x_{2}^{i}=k_{1}$ in order to guarantee stability. Using the stability criterion (3.13), Figure 3.2 is plotted as a function of $k_{2} / \omega$. We can see that $k_{2}$ is not necessarily the same as the bound of disturbance. If $k_{2}$ is 2 times of $\omega$, then $\mathrm{k}_{1}$ can be selected as small as $0.3^{*} \mathrm{~L} 2_{+}^{\mathrm{i}}$ as shown in Figure 3.2. 


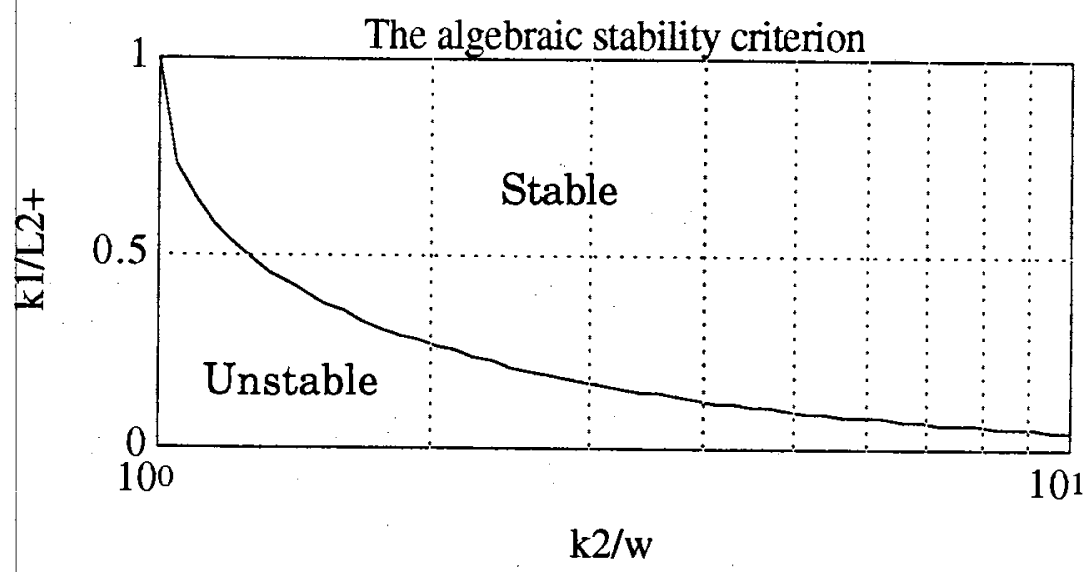

Figure 3.2 Stable region by the stability criterion (3.13)

\subsubsection{Example}

Let us consider a following second-order switching system

$$
\left\{\begin{array}{l}
\dot{x}_{1}=x_{2}-\operatorname{sgn}\left(x_{1}\right) \\
\dot{x}_{2}=-2 \operatorname{sgn}\left(x_{1}\right)+w
\end{array}\right.
$$

where $\mathrm{x}_{1}=0, \mathrm{x}_{2}^{\mathrm{i}}=\mathrm{L} 2_{+}^{\mathrm{i}}=5, \mathrm{w}_{1}=0.8^{*} \operatorname{sign}\left(\mathrm{x}_{2}\right), \mathrm{w}_{2}=0.5 * \operatorname{sign}\left(\mathrm{x}_{2}\right)$

The above initial condition satisfies the passing condition. Therefore, apply the stability criterion (3.13) and we have the necessary condition for stability:

$$
k_{2}=2, L 2_{+}=5\left\{\begin{array}{l}
\mathrm{w}_{1}=0.8 \rightarrow \mathrm{k}_{1}>1.044 \\
\mathrm{w}_{2}=0.5 \rightarrow \mathrm{k}_{1}>0.635
\end{array}\right.
$$




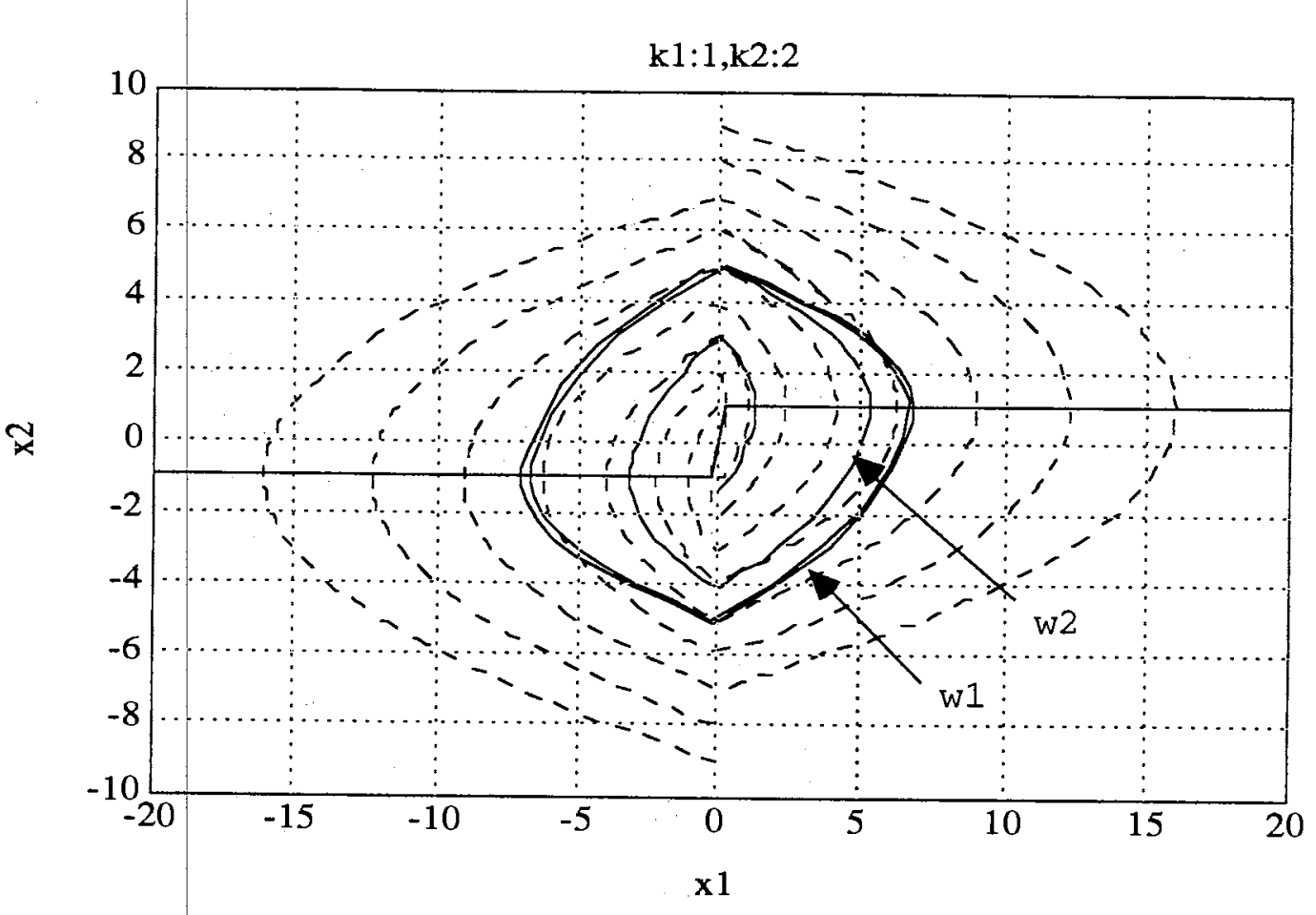

Figure 3.3 The template (dotted lines) and disturbed trajectories

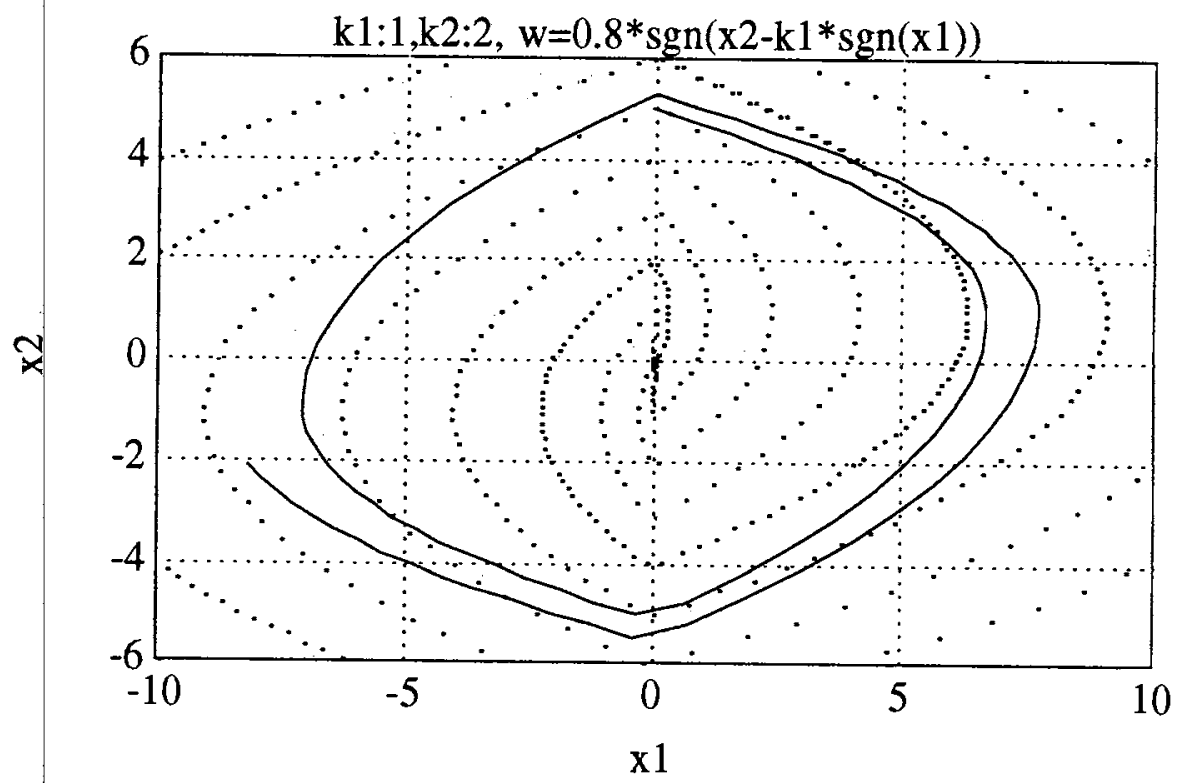

Figure 3.4 The worst case: $\mathrm{w}_{1}=0.8 * \operatorname{sign}\left(\mathrm{x}_{2}-\mathrm{k}_{1}{ }^{*} \operatorname{sgn}\left(\mathrm{x}_{1}\right)\right)$ 
The template (dotted lines) is made by the system without disturbance, and the disturbances for the trajectories (solid lines) are: $\mathrm{w}_{1}=0.8^{*} \operatorname{sign}\left(\mathrm{x}_{2}\right)$, $\mathrm{w}_{2}=0.5 * \operatorname{sign}\left(\mathrm{x}_{2}\right)$. The system's coefficient $\mathrm{k}_{1}=1$ is sufficient for the worst case of $\mathrm{w}_{2}=0.5$ : for the case of $\mathrm{w}_{2}=0.8$, it is not. In Figure 3.3, the disturbance $\mathrm{w}_{1}=0.8 * \operatorname{sign}\left(\mathrm{x}_{2}\right)$ forces the system to grow slowly. On the contrary, in Figure 3.4 , the worst disturbance $\mathrm{w}_{1}=0.8 * \operatorname{sign}\left(\mathrm{x}_{2}-\mathrm{k}_{1} * \operatorname{sgn}\left(\mathrm{x}_{1}\right)\right)$ forces the system to diverge faster than the former one does.

\subsection{Worst Direction in the Shifted-coordinate}

\subsubsection{Second-Order Reaching Dynamics}

Rewrite the equation (2.39) in Chapter 2 for a second-order case:

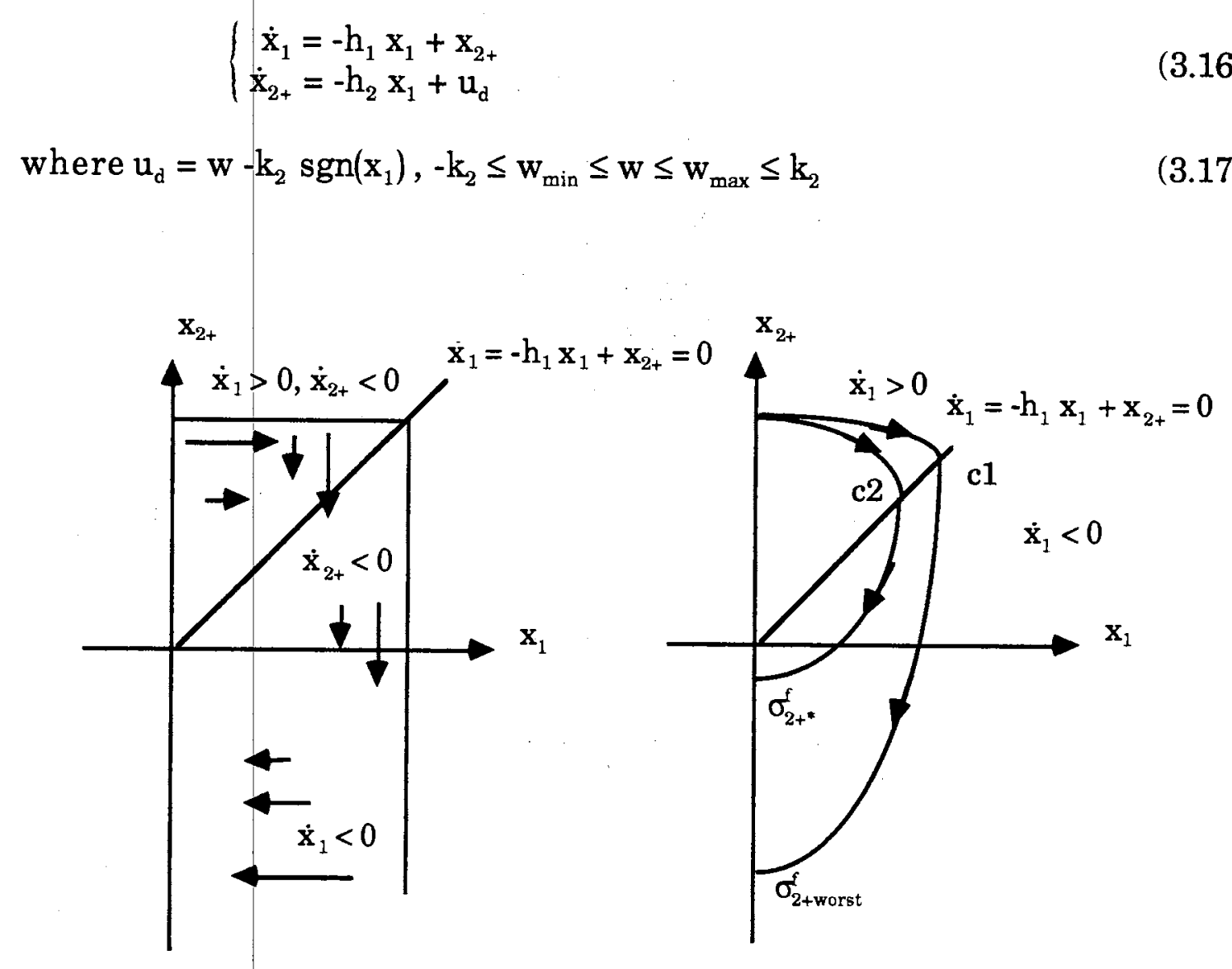

Figure 3.5 Velocity field and the worst trajectory 
For the region $\left(x_{1}>0 \cap \dot{x}_{1}>0\right)$, the worst direction is

$$
\begin{aligned}
\dot{\mathrm{x}}_{2+\text { worst }} & =\left.\dot{\mathrm{x}}_{2+}\right|_{\max \left(\dot{x}_{2}\right)} \\
& =\left.\dot{\mathrm{x}}_{2+}\right|_{\max (\omega)} \\
& =\left.\dot{\mathrm{x}}_{2+}\right|_{\left.\min \| \dot{x}_{2}\right)}\left(\because \dot{\mathrm{x}}_{2+}<0\right) \\
& =-\mathrm{h}_{2} \mathrm{x}_{1}
\end{aligned}
$$

With the passing condition, the solution point starts to move right. The maximum outermost point is $c 1$ which is determined by the slope $h_{1}$ and the coefficient $h_{2}$.

For the region $\left(x_{1}>0 \cap \dot{x}_{1}<0\right)$, the worst direction is

$$
\begin{aligned}
& \dot{\mathrm{x}}_{2+_{\text {worst }}}=\dot{\mathrm{x}}_{2+} \min _{\text {min }} \mid \\
& =\left.\dot{\mathrm{x}}_{2+}\right|_{\text {min(w) }} \\
& =\left.\dot{\mathrm{x}}_{2+}\right|_{\text {max } \| \dot{x}_{\mathrm{d}} \mid}\left(\because \dot{\mathrm{x}}_{2}<0\right) \\
& =-h_{2} x_{1}-2 k_{2}
\end{aligned}
$$

It is clear that any other trajectories are bounded by the worst trajectory, that the magnitude of the worst passing point is the bound of any other passing points.

$$
\left|\sigma_{2+\text { worst }}^{f}\right| \geq\left|\sigma_{2+*}^{f}\right|
$$

In the both half space, the worst disturbance is

$$
\mathrm{w}=\omega \operatorname{sgn}\left(\dot{\mathrm{x}}_{1}\right)
$$

This worst direction is the same as that of the switching system (see equation (3.8)).

\subsubsection{Worst Direction in the Velocity Field}

Let us consider the velocity field of a 2-dimensional space:

$$
\left\{\begin{array}{l}
\dot{x}_{1}=-h_{1} x_{1}+x_{2+} \\
\dot{x}_{2+}=-h_{2} x_{1}+u_{d}
\end{array}\right.
$$


where $u_{d}=w-k_{2} \operatorname{sgn}\left(x_{1}\right),-k_{2} \leq w_{\min } \leq w \leq w_{\max } \leq k_{2}$

In order to specify the coordinate transformation, the subscript + or are used in the dynamics equations. However, for convenience, the subscript can be ignored without confusion.

At the point $P_{1}$ on the trajectory in Figure 3.6 (a), the disturbance input does not change the difference $d x_{1}$ but change the difference $d x_{2}, e . g . d x_{2}$ (1) and $d x_{2}$ (2). The sign of the velocity direction $d x_{2}$ is strictly negative according (3.17). The worst case trajectory is defined as a trajectory that encloses any other trajectory with the same bounded disturbance in the phase plane. If a moving point moves outward always, then it will compose the worst trajectory for a given initial condition.

Even though the finite next point $P_{2}^{\circledast}$ is outward compared to the point $P_{2}^{\oplus}$, the distance of $P_{2}^{\circledast}$ from the origin itself is closer than the other point.

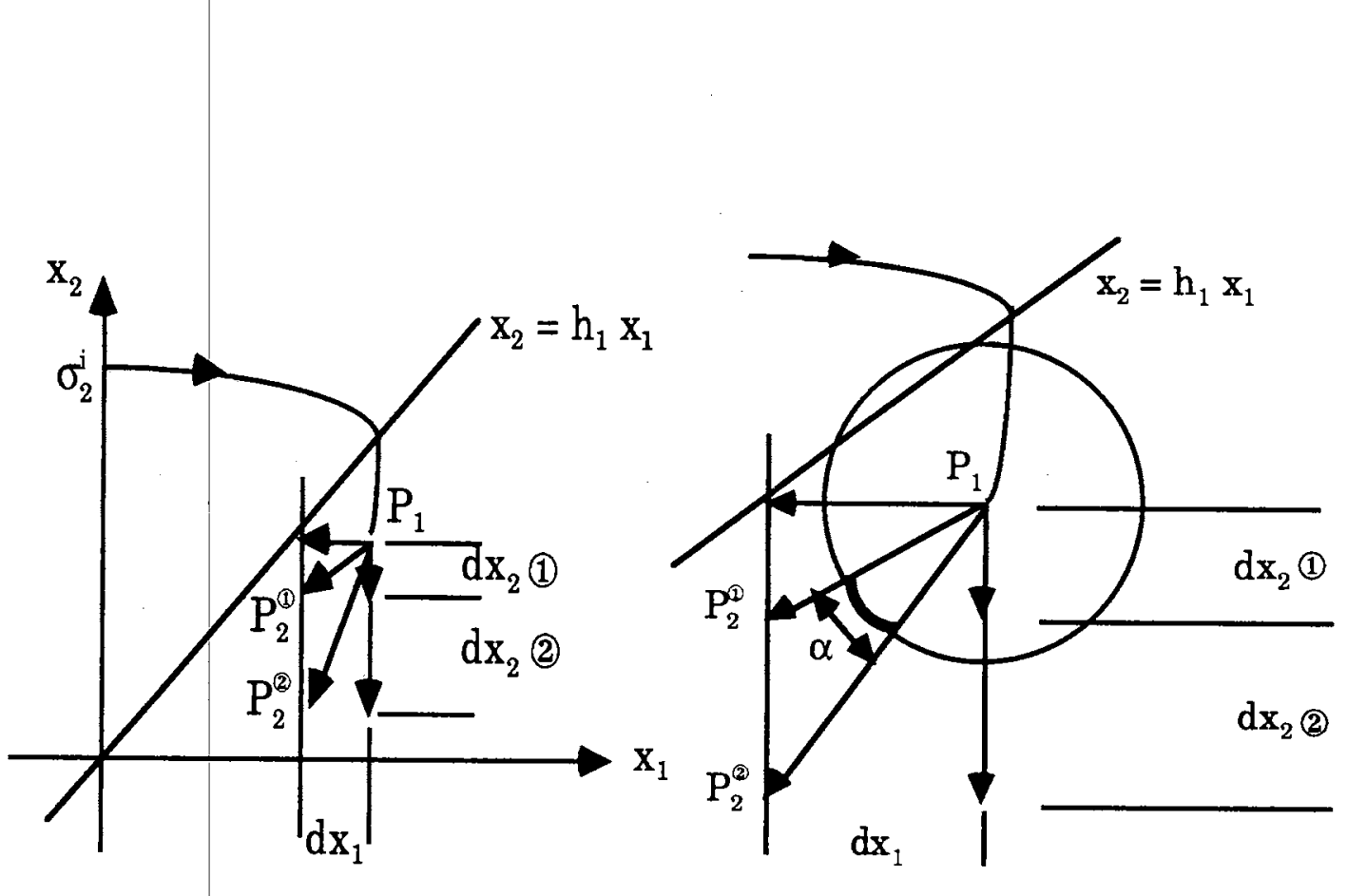

(a)

(b)

Figure 3.6 (a) Conception of velocity field (b) The fictitious next point 
By adding the scaled differences of $d x_{1}$ and $d x_{2}$ to the point $P_{1}$, fictitious next point $\mathrm{P}_{2^{*}}$ is defined as follows:

$$
\mathrm{P}_{2^{*}}=\mathrm{P}_{2^{*}}\left(\mathrm{x}_{1^{*}}(\mathrm{t}+\mathrm{dt}), \mathrm{x}_{2^{*}}(\mathrm{t}+\mathrm{dt})\right)
$$

where $x_{1^{*}}(t+d t)=x_{1}(t)+\frac{d x_{1}}{\delta \xi}$

$$
\mathrm{x}_{2^{*}}(\mathrm{t}+\mathrm{dt})=\mathrm{x}_{2}(\mathrm{t})+\frac{d \mathrm{x}_{2}}{\delta \xi}
$$

$$
\delta \xi=C_{s} \sqrt{\left(\mathrm{dx}_{1}\right)^{2}+\left(\mathrm{dx}_{2}\right)^{2}} \text { and } \mathrm{C}_{\mathrm{s}} \text { is a constant. }
$$

It is clear that the outmost fictitious points compose the worst trajectory. The generalization of the fictitious next point is obvious and the direction of worst fictitious point is the worst direction of disturbance input.

$$
\left\{\begin{array}{c}
\mathrm{x}_{1^{*}}(\mathrm{t}+\mathrm{dt})=\mathrm{x}_{1}(\mathrm{t})+\frac{d \mathrm{x}_{1}}{\delta \xi} \\
\mathrm{x}_{2^{*}}(\mathrm{t}+\mathrm{dt})=\mathrm{x}_{2}(\mathrm{t})+\frac{d \mathrm{x}_{2}}{\delta \xi} \\
\cdots \\
\mathrm{x}_{\mathrm{n}^{*}}(\mathrm{t}+\mathrm{dt})=\mathrm{x}_{\mathrm{n}}(\mathrm{t})+\frac{d \mathrm{x}_{\mathrm{n}}}{\delta \xi}
\end{array}\right.
$$

where $\delta \xi=C_{s} \sqrt{\left(\mathrm{dx}_{1}\right)^{2}+\left(\mathrm{dx}_{2}\right)^{2}+\ldots+\left(\mathrm{dx}_{\mathrm{n}}\right)^{2}}$ and $\mathrm{C}_{\mathrm{s}}$ is a constant.

For the second-order case, the fictitious next points e.g. $\mathrm{P}_{2}^{\Phi}, \mathrm{P}_{2}^{\circledast}$ lie on the arc $\alpha$ in Figure 3.6 (b) which is less than the quarter of the peripheral of the unit circle centered at $P_{1}$ because the sign of $d x_{n}$ is strictly negative and $d x_{1}$ is fixed as positive or negative.

For the general n_th-order case, the fictitious next points composes a line on the $n_{-}$th-order sphere surface since all the different components $d x_{i}$ except $d x_{n}$ are determined by only $x(t)$ and $d x_{n}$ is strictly negative and bounded. In the phase plane of $x_{n}$ and $x_{1}$, the fictitious next point is less than the 
quarter of the peripheral of the unit circle centered at $P_{1}$ also. Since the direction of quarter line is function of $\dot{\mathbf{x}}_{1}, \dot{\mathbf{x}}_{2}, \ldots, \dot{\mathbf{x}}_{\mathrm{n}-1}$, it is not easy to visualize the worst direction as the second-order case. Hence, it is proper to search the worst direction by numeric simulation.

\subsubsection{Numerical Search}

The worst bound by the disturbance input can be interpreted as the problem where the distance (or the Lyapunov-like function) has to be maximized based on the evaluations of the results from several simulations of the bounded disturbance input. The optimization is done by the Goldensection search that does not require the derivatives of the function. It is more reliable but slower method. Suppose that the disturbance input in (3.17) is a real valued function defined on $\left[-2 k_{n}, 0\right]$. The distance from the shifted origin or the Lyapunov-like function can be the cost function. It is reasonable to assume that the cost function is unimodal and its graph takes one of the three forms as shown in Figure 3.7 The interval [0,2] in Figure 3.7 is not essential but convenient to implement the sliding observer. The Golden-section search algorithm is optimal in the sense that it uses the least number of evaluations of the cost function for a desired accuracy.
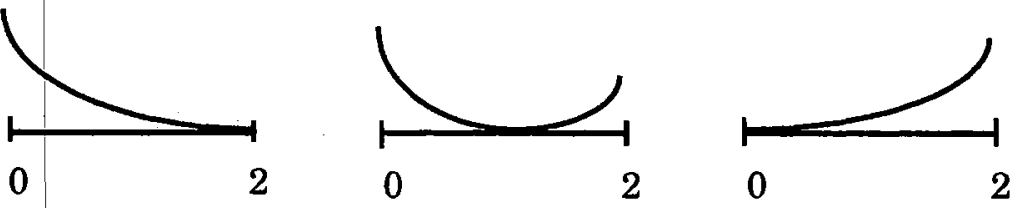

Figure 3.7 Unimodal functions 
Structure of the Code. The worst direction of the sliding observer should be evaluated by simulation for each value of the disturbance input. The different simulations are used by the search procedure. The optimization algorithm is written as a discrete time system to control each simulation conveniently. Thus, the many simulations are integrated to one long worst simulation. Three subsystems are connected by the connecting system CONN.T. They are: SYS.T, which contains the sliding observer error dynamics, the search algorithm GOLD.T, and the cost function COST.T. Each step in the search starts with a given value of the disturbance input from the GOLD.T, then the response of the SYS.T is evaluated by COST.T. The value $J\left(U_{d}\right)$ is obtained at the end of the each step GOLD.T, then the value $J\left(U_{d}\right)$ is used to calculate the next value of $U_{d}$. The initial values of SYS.T and COST.T are reset by GOLD.T. (See detail Appendix C.5).

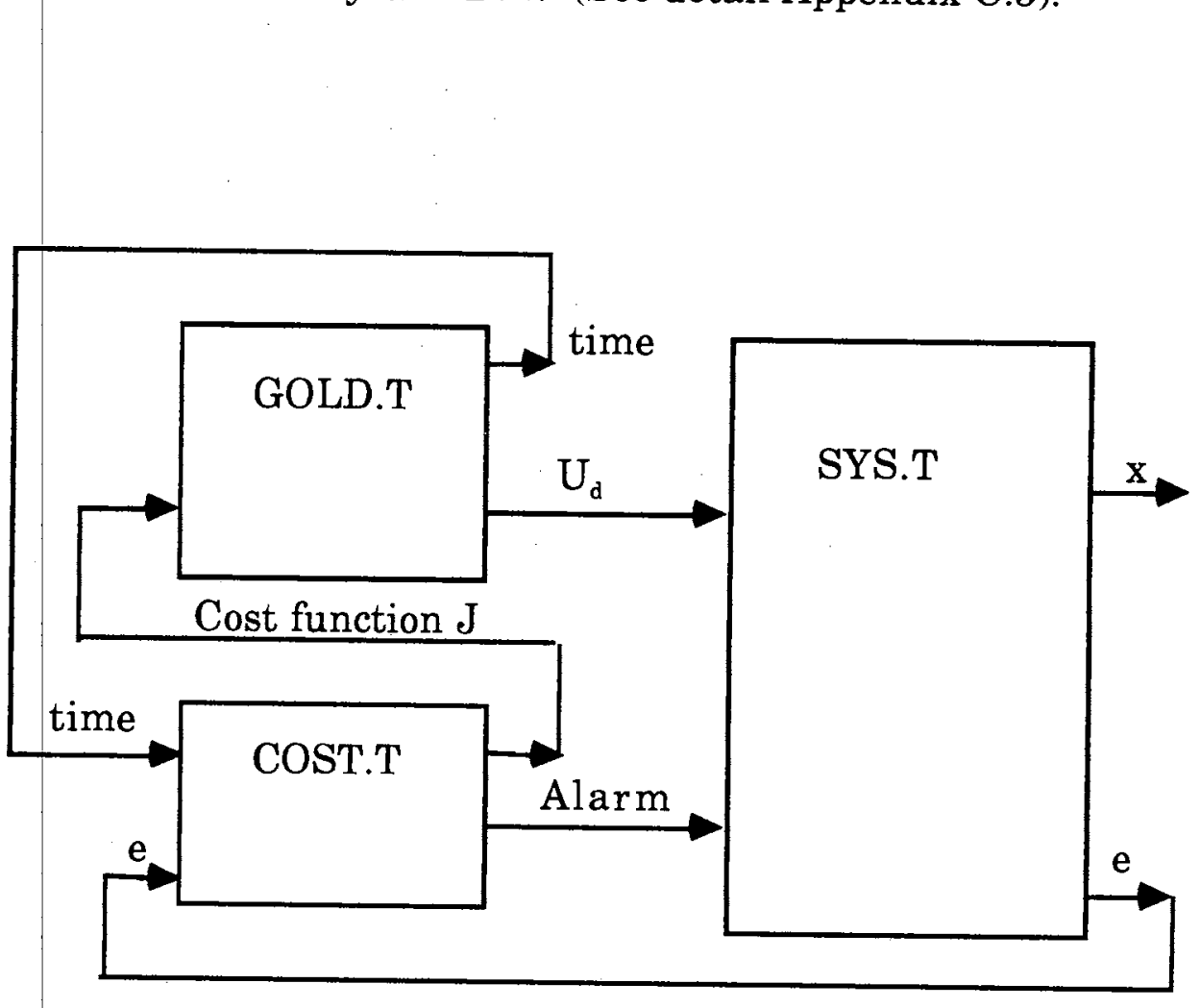

Figure 3.8 Block diagram of the optimization algorithm 
Example 3.1 The simulation results of the reaching dynamics with $\mathrm{H}=[1.8 .95 .25]^{\mathrm{T}}, \mathrm{k}_{\mathrm{n}}=0.023$.
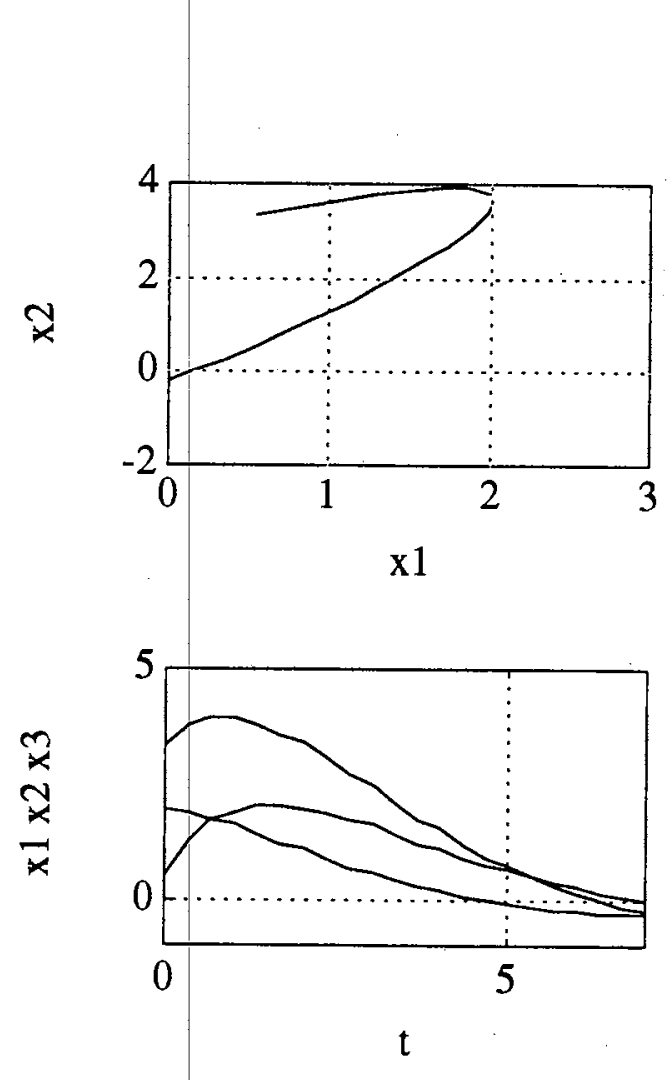
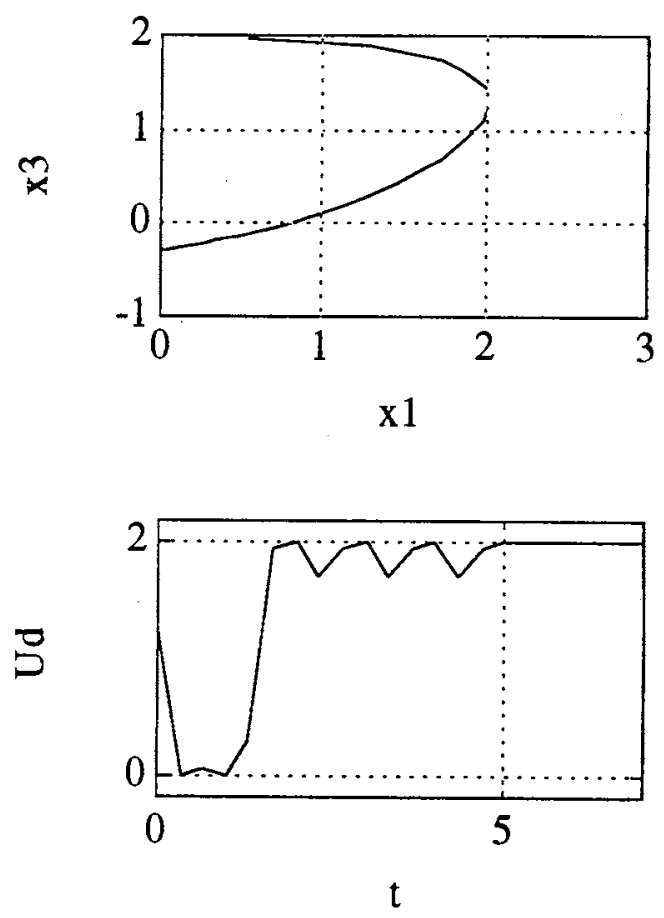

Figure 3.9 The simulation result of the numeric search

The state $x_{1}$ is maximum at $x_{2}=h_{1} x_{1}$ and in about 1.7 second. The worst direction by the numerical search is approximately $w=\operatorname{sign}\left(\dot{\mathrm{x}}_{1}\right)$ which is the same as the 2-order case.

\subsubsection{Approximate Worst Direction}

For the second-order case, the worst direction is analytically obtained. For the higher dimensional cases, it is difficult to define the outward direction, so that, theoretically, a numerical search is proposed in the previous section. 
However, the numeric search for the high dimensional space takes much more computational efforts. Hence, it is reasonable to find an approximate worst direction for a practical design purpose.

Consider a third-order reaching dynamics in the shifted-coordinate. It satisfies the passing condition and it is a linear system until it reaches the hyperplane.

$$
\left\{\begin{array}{l}
\dot{x}_{1}=-h_{1} x_{1}+x_{2+} \\
\dot{x}_{2+}=-h_{2} x_{1}+x_{3+} \\
\dot{x}_{3+}=-h_{3} x_{1}+u_{d}
\end{array}\right.
$$

where $u_{d}=w-k_{3} \operatorname{sgn}\left(x_{1}\right),-k_{3} \leq w_{\min } \leq w \leq w_{\max } \leq k_{3}$

From the equation (3.24), even though the velocity $\dot{\mathrm{x}}_{1}$ and $\dot{\mathrm{x}}_{2+}$ are function of the position only, the trajectory of moving point is also a function of the disturbance input.

For the region $\left(x_{1}>0 \cap \dot{x}_{1}>0\right)$, in the $x_{1}-x_{2}$ phase plane, the equation (3.24) is rewritten as:

$$
\left\{\begin{array}{l}
\dot{x}_{1}=-h_{1} x_{1}+x_{2+} \\
\dot{x}_{2+}=-h_{2} x_{1}+\sigma_{3+}^{i}-h_{3} \int_{0}^{t} x_{1} d t+\int_{0}^{t} u_{d} d t
\end{array}\right.
$$

For the region, the worst direction of $\dot{\mathbf{x}}_{2}$ is approximately the same as the maximized velocity $\dot{x}_{2}$ by the disturbance input. Because the disturbance input is strictly negative, the maximum of time integral of the disturbance input in (3.25) is zero. 


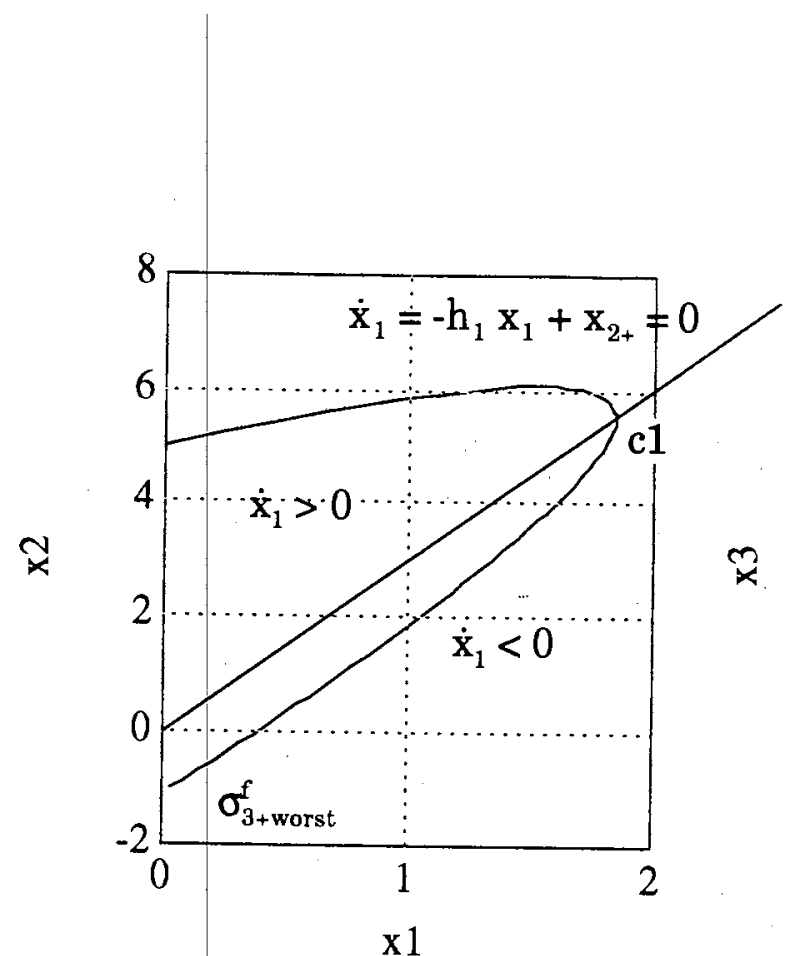

Figure 3.10 The trajectory of solution point and velocity field

Along the worst trajectory, the velocity of moving point is

$$
\begin{aligned}
\dot{\mathrm{x}}_{2+{ }_{\text {worst }}} & =\dot{\mathrm{x}}_{2+} \mid \max \left(\dot{\mathrm{x}}_{2+}\right) \\
& =\dot{\mathrm{x}}_{2+} \mid \max \left(\mathrm{x}_{3+}\right)
\end{aligned}
$$

When the trajectory pass through the line $\dot{\mathrm{x}}_{1}=0$, i.e., at the changing point $c 1$, the velocity direction of $\dot{x}_{1}$ changes. The worst direction disturbance pushes the change point $\mathrm{c} 1$ off from the origin point. When $\dot{\mathrm{x}}_{3}$ is maximum i.e. $u_{d}=0$, the $x_{3}$ state is maximized. Since $\dot{x}_{1}>0, w=\omega \operatorname{sgn}\left(\dot{x}_{1}\right)$ makes $u_{d}=0$ by (3.17)

$$
\begin{aligned}
\dot{\mathrm{x}}_{3+\text { worst }} & =\left.\dot{\mathrm{x}}_{3+}\right|_{\max \left(\dot{x}_{3}\right)}\left(\because \dot{\mathrm{x}}_{3+}<0\right) \\
& =\left.\dot{\mathrm{x}}_{3+}\right|_{\max (w)} \\
& =-\mathrm{h}_{3} \mathrm{x}_{1}
\end{aligned}
$$

For the region $\left(x_{1}>0 \cap \dot{x}_{1}<0\right)$, along the worst trajectory in the $x_{1}-x_{2}$ 
phase plane, the velocity of moving point is

$$
\dot{\mathbf{x}}_{2+_{\text {worst }}}=\dot{\mathrm{x}}_{2+} \mid \min \left(x_{9}\right)
$$

To minimize $x_{3}$, the worst velocity direction is approximately:

$$
\begin{aligned}
\dot{\mathrm{x}}_{3+\text { worrt }} & =\left.\dot{\mathrm{x}}_{3+}\right|_{\min \left(\dot{x}_{3}\right)}\left(\because \dot{\mathrm{x}}_{3+}<0\right) \\
& =\dot{\mathrm{x}}_{3+} \mid \min (\mathrm{w}) \\
& =-\mathrm{h}_{3} \mathrm{x}_{1}-2 \mathrm{k}_{3}
\end{aligned}
$$

Since the velocity of $\dot{x}_{2}$ is function of integral of disturbance input as the equation (3.26), the minimum velocity might be obtained by minimum disturbance input. However, the worst changing point $\mathrm{c} 1$ is obtained already by the maximum disturbance input as the equation (3.27). Hence, the direction is only approximation of the worst case.

In the both half space, the approximate worst disturbance is

$$
w=\omega \operatorname{sgn}\left(\dot{x}_{1}\right)
$$

which is the same as the second-order case. Even though the exact worst direction is function of all states, the disturbance effect in the $x_{1}-x_{2+}$ space was considered only in the approximate worst direction method.

Generalization of Approximate Worst Direction. Consider a n_th-order reaching dynamics in the shifted-coordinate. We can assume that it satisfies the passing condition without loss of generality.

$$
\left\{\begin{array}{c}
\dot{x}_{1}=-h_{1} x_{1}+x_{2+} \\
\dot{x}_{2+}=-h_{2} x_{1}+x_{3+} \\
\cdots \\
\dot{x}_{n+}=-h_{n} x_{1}+u_{d}
\end{array}\right.
$$

Since the passing state is not desirable than the sliding state and the 
sliding condition(2.9) is a function of the state $\mathbf{x}_{2}$ only, the state $\mathbf{x}_{2}$ is more important than the other states except $x_{1}$. Hence, it is reasonable to define the worst direction of disturbance as a disturbance that makes the state $x_{2}$ worst or equivalently maximizes $\left|\sigma_{2}^{f}\right|$.

Each state is obtained as time functions as follows:

$$
\begin{aligned}
x_{2+}(t) & =\sigma_{2+}^{i}-h_{2} \int_{0}^{t} x_{1} d \tau+\int_{0}^{t} x_{3+} d \tau \\
x_{3+}(t) & =\sigma_{3+}^{i}-h_{3} \int_{0}^{t} x_{1} d \tau+\int_{0}^{t} x_{4+} d \tau \\
\cdots & \\
x_{n+}(t) & =\sigma_{n+}^{i}-h_{n} \int_{0}^{t} x_{1} d \tau+\int_{0}^{t} u_{d} d \tau
\end{aligned}
$$

By plugging the equation (3.32) into the simultaneous differential equation (3.31), we obtains the following equation. According to the notational definition (2.58), the initial states $x^{i}$ is denoted as $\sigma^{i}$ to emphasize that they are constants.

$$
\begin{aligned}
\dot{x}_{1}=-h_{1} x_{1} & +x_{2+} \\
\dot{x}_{2+}=-h_{2} x_{1} & +\sigma_{3+}^{i}-h_{3} \int_{0}^{t} x_{1} d \tau \\
& +\int_{0}^{t} \sigma_{4+}^{i}(d \tau)-h_{4} \int_{0}^{t} x_{1}(d \tau)^{2} \\
& + \\
& +\int_{0}^{t} \sigma_{n+}^{i}(d \tau)^{n-3}-h_{n} \int_{0}^{t} x_{1}(d \tau)^{n-2}+\int_{0}^{t} u_{d}(d \tau)^{n-2}
\end{aligned}
$$

where $\int x_{1}(d t)^{n}$ is n-multiple integral and $u_{d}=w-k_{n} \operatorname{sgn}\left(x_{1}\right)$, $-\mathrm{k}_{\mathrm{n}} \leq \mathrm{w}_{\min } \leq \mathrm{w} \leq \mathrm{w}_{\max } \leq \mathrm{k}_{\mathrm{n}}$. 
In the $x_{1}-x_{2+}$ phase plane, the solution point of the general dimensional case behaves according to equation (3.33). For the short period $(t<<1)$, the integral of disturbance input hardly affects the velocity direction of the state $\mathbf{x}_{2}$ comparing to other terms. Since the disturbance input alters the velocity direction of the lower state via the integrators, for the short period, it changes the velocity direction of $x_{n}$ only. For the case of positive initial state of $x_{n+}$, the worst direction in $\mathbf{x}_{1}-\mathbf{x}_{\mathrm{n}+}$ phase plane can be obtained as the same way as the case of 2-dimension. However, for the case of negative initial state of $\mathrm{x}_{\mathrm{n}+}$, the worst direction in $\mathrm{x}_{1}-\mathrm{x}_{\mathrm{n}+}$ phase plane cannot be defined as clearly as the case of 2-dimension.

Let us consider a long period motion of solution point. Since the integrator order of disturbance input is the highest term of the equation (3.33), the velocity direction of the state $x_{2}$ will be affected mostly by the disturbance input. For a long period, the approximate worst direction can be obtained as the same method as the 3- dimensional case.

For the region $\left(\mathrm{x}_{1}>0 \cap \dot{\mathrm{x}}_{1}>0\right)$, the approximate worst direction is

$$
\begin{aligned}
\dot{\mathrm{x}}_{2+\text { worst }} & =\dot{\mathrm{x}}_{2+} \mid \max \left(\dot{x}_{2}\right) \\
& =\left.\dot{\mathrm{x}}_{2+}\right|_{\max (w)}
\end{aligned}
$$

For the region $\left(\mathrm{x}_{1}>0 \cap \dot{\mathrm{x}}_{1}<0\right)$, the approximate worst direction is

$$
\begin{aligned}
\dot{\mathrm{x}}_{2+\text { worst }} & =\dot{\mathrm{x}}_{2+} \mid \min \left(\dot{x}_{2}\right) \\
& =\left.\dot{\mathrm{x}}_{2+}\right|_{\min (w)}
\end{aligned}
$$

For the both cases, the approximate worst disturbance is

$$
\mathbf{w}=\omega \operatorname{sgn}\left(\dot{\mathbf{x}}_{1}\right)
$$

which is the same as the second- or third-order cases. 


\subsubsection{General Remarks}

Usually, it was conjectured that the second-order switching system is globally stable with the bounded disturbance condition, i.e., $\mathrm{k}_{2}>|\mathrm{w}|$. In the worst analysis, the algebraic stability condition (3.13) shows that the switching system is not globally stable and it depends on the initial condition and the design constants $k_{1}$ and $k_{2}$. The shearing effects (3.11) and (3.12) explain why the sliding observer converges faster than the linear observers do. In the second-order phase plane analysis, it is clear that the shiftedcoordinate system brings the shearing effect. By introducing the coordinate transformation, the worst case analysis shows that the switching terms shift the coordinate and bring the shearing effect. However, we should notice that these effects are finite and constant, i.e., $k_{1}, k_{2}$, etc. These switching terms are compared to the linear correction terms that have relatively small effect near the origin but have proportional effect to the state $x_{1}$. The necessity of linear correction terms are already showed in the reaching dynamics (see (2.23)). Roughly speaking, the second-order sliding observer stability is: if the state divergence due to the worst disturbance is less than the shearing effect plus the convergence by linear correction, then it is stable. For higher dimensional system, phase plane analysis is not sufficient to explain the "general shearing effect." Therefore, we need another tool to measure the effects and to explain stability.

\subsection{Lyapunov-like Function}

\subsubsection{Introduction}

This chapter focuses on the stability analysis of the sliding observer. 
The scheme to analyze the stability is as follows: First, when the sliding observer does not satisfy the sliding condition, it passes through the hyperplane until satisfying the sliding condition. By applying the shiftedcoordinate system, the sliding observer is transformed to a linear system with disturbance input, i.e., reaching dynamics. Second, after satisfying the sliding condition, it becomes a reduced order linear system, i.e., Filippov's equivalent dynamics. This chapter investigates the stability of the sliding observer by using a Lyapunov-like function which describes a fictitious energy, i.e., so called "pseudo-energy" of a system. The employment of quadratic Lyapunov-like function makes easy the whole domain to separate into regions where, with the passing condition, two reaching dynamics are activated and, with the sliding condition, the reduced order sliding dynamic is turned on. Each coordinate associated with switching has its own a Lyapunov-like function of the form $V_{s}=x_{s}^{T} P_{s} x_{s}(s=+,-)$ where $P_{s}$ is a real symmetric positive definite (r.s.p.d) matrix. Roughly speaking, if the pseudoenergy of each dynamics is strictly decreasing along the trajectories in the accompanied successive coordinate systems, then the sliding observer is stable. The shearing effect due to the shifted-coordinate characterizes the sliding observer. The difference of Lyapunov-like functions of successive switching coordinates is due to the shearing effect. Generalization of shearing effect is explained by several intriguing system properties.

\subsubsection{Lyapunov-like Function Theorem}

A quadratic function of the form, $\mathrm{x}^{\mathrm{t}} \mathrm{P} \mathrm{x}$, are common form of a Lyapunov function used for investigating stability questions of linear autonomous systems, i.e., $\dot{x}(t)=A x(t)$ [Kailath, 1980]. Since the system with $u_{d}=0$ is 
equivalent to the composition of the three linear autonomous systems, i.e., $\dot{x}_{s}(t)=A_{s} x_{s}(t)$ (subscript $s=+,-, 0$ ), it would be natural to use a quadratic function as representing the Lyapunov-like function[Alimov, 1960]. Hence, it is necessary to delineate the composite structure of $V_{s}\left(x_{+}\right)=x_{s}^{T} P x_{s}$ in the domain of $\Omega_{\mathrm{s}}$ (subscript $\mathrm{s}=+,-, 0$ )[Peleties, 1991].

Definition 3.1 A scalar continuous function $\mathrm{V}(\mathrm{x})$ is said to be locally positive definite if $\mathrm{V}(0)=0$ and, in a ball $\mathrm{B}_{\mathrm{R}_{\mathrm{o}}}$ [Slotine, 1991].

$$
\mathrm{x} \neq 0 \Rightarrow \mathrm{V}(\mathrm{x})>0
$$

If $\mathrm{V}(0)=0$ and the above property holds over the whole state space the $\mathrm{V}(\mathrm{x})$ is said to be globally positive definite.

Definition 3.2 The function $\mathrm{V}_{\mathrm{s}}(\mathrm{x})$ is said to be radially unbounded if

$$
\lim _{\left\|x_{s}\right\| \rightarrow \infty} V_{s}\left(x_{s}\right) \rightarrow \infty
$$

Definition 3.3 A linear system $\dot{\mathrm{x}}(\mathrm{t})=\mathrm{Ax}(\mathrm{t})+\mathrm{b} \mathrm{u}(\mathrm{t}), \mathrm{y}(\mathrm{t})=\mathrm{cx}(\mathrm{t})$ is internally stable or stable in the sense of Lyapunov if the solution of $\dot{x}(t)=A x(t), \quad x\left(t^{i}\right)=x_{0}, t \geq t^{i}$ tends toward zero as $t \rightarrow \infty$ for arbitrary $x_{0}$.

Definition 3.4 A set $\mathcal{R}$ is said to be a cone if $\forall \mathrm{x}_{\mathrm{s}} \in \mathcal{R}, \alpha \mathrm{x}_{\mathrm{s}} \in \mathcal{R}, \forall \alpha>0$.

Definition 3.5 Let us define a domain $\Omega_{+}$where $\mathrm{x}_{1}>0$ as right half space and a domain $\Omega$. where $\mathrm{x}_{1}<0$ as left half space in each shifted-coordinate system. A hyperplane domain $\Omega_{0}$ where $x_{1}=0$ and $\left|x_{2}\right| \leq k_{1}$ in the original coordinate is said sliding zone. The whole domain is as follow:

$$
\Omega=\Omega_{+} \cup \Omega_{-} \cup \Omega_{\mathrm{o}} \subset \mathbb{R}^{\mathrm{n}}
$$

Observe that both $\Omega_{+}$and $\Omega_{-}$are cones. If $\mathbf{x}_{\mathrm{s}}$ is a member of $\Omega_{\mathrm{s}}$, then any 
positive scalar multiple $\alpha$ of $x_{s}$ is also a member of $\Omega_{\mathrm{s}}$. Neither right or left reaching dynamics has equilibrium point within the domain of $\Omega_{\mathrm{s}}$.

Corollary 3.1 If $\mathrm{V}_{\mathrm{s}}\left(\mathrm{x}_{\mathrm{s}}\right)=\mathrm{x}_{\mathrm{s}}^{\mathrm{T}} \mathrm{P} \mathrm{x}_{\mathrm{s}}$ then every region $\mathrm{x}_{\mathrm{s}}$ is a cone [Peleties, 1991].

Proof) Let assume $x_{s}^{\prime} \in \Omega_{s}$. The time derivative of $V_{s}$ is

$$
\dot{\mathrm{V}}_{\mathrm{s}}\left(\mathrm{x}_{\mathrm{s}}^{\prime}\right)=\frac{\partial \mathrm{V}_{\mathrm{s}}\left(\mathrm{x}_{\mathrm{s}}\right)}{\partial \mathrm{x}} \mathrm{A}_{\mathrm{s}} \mathbf{x}_{\mathrm{s}} \mathrm{I}_{\mathrm{x}_{\mathrm{g}}=\mathbf{x}_{\mathrm{s}}^{\prime}}=\left.\frac{\partial \mathrm{V}_{\mathrm{s}}\left(\mathrm{x}_{\mathrm{s}}\right)}{\partial \mathrm{x}}\right|_{\mathbf{x}_{\mathrm{g}}=\mathbf{x}_{\mathrm{s}}^{\prime}} \mathrm{A}_{\mathrm{s}} \mathrm{x}_{\mathrm{s}}^{\prime} \leq 0 \text {. }
$$

Consider $x^{\prime \prime}=\alpha x^{\prime}$. Then the time derivative of $V_{s}$ indicates that $x^{\prime \prime} \in \Omega_{s}$ as follows:

$$
\dot{V}_{s}\left(x_{s}^{\prime \prime}\right)=\left.\frac{\partial V_{s}\left(x_{s}\right)}{\partial x} A_{s} x_{s}\right|_{x_{g}=x_{s}^{*}}=\left.\alpha \frac{\partial V_{s}\left(x_{s}\right)}{\partial x}\right|_{i_{s}=x_{s}^{\prime}} A_{s} \alpha x_{s}^{\prime}=\left.\alpha^{2} \frac{\partial V_{s}\left(x_{s}\right)}{\partial x}\right|_{x_{8}=x_{s}^{\prime}} A_{s} x_{s}^{\prime} \leq 0
$$

Note that the above is true for any value of $\alpha$, therefore the positive values of $\alpha$ satisfy Definition 3.4.

Corollary 3.2 Let $\left(\lambda_{j}, e_{j}\right)$ be an eigenvalue/ eigenvector pair of $A_{m}$. Then the derivative of Lyapunov-like function is negative definite $\left(\dot{V}_{s}\left(e_{j}\right)<0\right)$ if and only if $\operatorname{Re}\left(\lambda_{\mathrm{j}}\right)<0$ [Peleties, 1991].

Proof) Let assume that $\dot{V}_{s}\left(e_{j}\right)<0$. Then it can be rewrite as follows:

$$
\begin{aligned}
& \dot{V}_{s}\left(e_{j}\right)=e_{j}^{T}\left(A_{m}^{T} P+P A_{m}\right) e_{j}=e_{j}^{T} A_{m}^{T} P e_{j}+e_{j}^{T} P A_{m} e_{j} \\
& =\bar{\lambda}_{j} e_{j}^{T} P e_{j}+\lambda_{j} e_{j}^{T} P e_{j}=\left(\bar{\lambda}_{j}+\lambda_{j}\right) V_{s}\left(e_{j}\right)=2 \operatorname{Re}\left(\lambda_{j}\right) V_{s}\left(e_{j}\right)<0(=0)
\end{aligned}
$$

A quadratic Lyapunov function is positive definite:

$$
\mathrm{V}_{\mathrm{s}}\left(\mathrm{x}_{\mathrm{s}}\right)>0, \mathrm{x}_{\mathrm{s}} \neq 0, \forall \mathrm{x}_{\mathrm{s}} \in \mathrm{C}^{\mathrm{n}}
$$

Since $V_{s}\left(e_{j}\right)>0$, the other term must be negative $\left(\operatorname{Re}\left(\lambda_{j}\right)<0\right)$. 
Let assume $\operatorname{Re}\left(\lambda_{\mathrm{j}}\right)<0$. Applying similar procedures, we have the same results: $\dot{\mathrm{V}}_{\mathrm{s}}\left(\mathrm{e}_{\mathrm{j}}\right)=2 \operatorname{Re}\left(\lambda_{\mathrm{j}}\right) \mathrm{V}_{\mathrm{s}}\left(\mathrm{e}_{\mathrm{j}}\right)$. Since $\mathrm{V}_{\mathrm{s}}\left(\mathrm{e}_{\mathrm{j}}\right)>0$ then $\dot{\mathrm{V}}_{\mathrm{s}}\left(\mathrm{e}_{\mathrm{j}}\right)<0$.

Corollary 3.3 A solution trajectory of system $A_{m}$ cannot escape to infinity within $\Omega_{\mathrm{s}}$.

Proof) Assume a solution point of system $A_{m}$ escape to infinity within $\Omega_{s}$, i.e., $\lim _{t \rightarrow \infty} x_{s}\left(t ; x_{0}, \tau_{0}\right)=\infty$, then $\exists t_{2}>t_{1} \geq \tau_{0}$ such that $V_{s}\left(x_{s}\left(t_{2}\right)\right)>V_{s}\left(x_{s}\left(t_{1}\right)\right)$ where $\quad x_{s}(t) \equiv x_{s}\left(t ; x_{s}^{i}, \tau^{i}\right) . \quad$ However, $\quad V_{s}\left(x_{s}\left(t_{2}\right)\right)=V_{s}\left(x_{s}\left(t_{1}\right)\right)+\int_{t_{1}}^{t_{2}} \dot{V}_{s}\left(x_{s}(q)\right) d q$ implies that $\int_{t_{1}}^{t_{2}} \dot{V}_{i}\left(x_{s}(q)\right) d q>0$ which is clearly a contradiction of the fact that $\dot{\mathrm{V}}_{\mathrm{s}}\left(\mathrm{x}_{\mathrm{s}}(\mathrm{q})\right)<0, \forall \mathrm{x}_{\mathrm{s}}(\mathrm{t}) \in \Omega_{\mathrm{s}}, \mathrm{s}=+.-.0$.

The definitions and propositions give us the general pictures of the dynamics of system $A_{m}$ within $\Omega_{\mathrm{s}}$. We have established that there are no equilibrium points within $\Omega_{\mathrm{s}}$ and in addition the trajectories will not go to infinity. This implies that the trajectories will either asymptotically approach the shifted origin or they will approach the sliding patch. The trajectories will enter the sliding patch where sliding can take place so that the Filippov's equivalent dynamics will describe the behavior of the error dynamics.

\subsubsection{Passing Jump of Lyapunov-like Function}

A Lyapunov-like function candidate is a quadratic form, $V_{s}=x_{s}^{T} P x_{s}$ which is not a continuous function and does not satisfy the usual requirement of Lyapunov function. This function has several interesting features that can be interpreted as a good nature. Whereas the usual Lyapunov function shows continuity, Lyapunov-like function in the shifted-coordinate shows a 
discontinuity ("passing jump") between the initial and final states on the hyperplane.

\section{Relations Between the Passing Points.}

i) Passing right case (with the passing condition $x_{1}=0, x_{2}>k_{1}$ ):

The passing points relations in the shifted-coordinate are

$$
\mathbf{s}_{+}^{\mathrm{i}}\left(\tau_{\mathrm{j}}\right)=\mathrm{s}_{-}^{\mathrm{f}}\left(\tau_{\mathrm{j}}\right) \Rightarrow \sigma_{+}^{\mathrm{i}}\left(\tau_{\mathrm{j}}\right)=\sigma_{-}^{\mathrm{f}}\left(\tau_{\mathrm{j}}\right)-2 \mathrm{~K}_{\mathrm{s}}
$$

ii) Passing left case (with the passing condition $x_{1}=0, x_{2}<-k_{1}$ ):

The passing points relations in the shifted-coordinate are

$$
\mathrm{s}_{-}^{\mathrm{i}}\left(\tau_{\mathrm{j}}\right)=\mathrm{s}_{+}^{\mathrm{f}}\left(\tau_{\mathrm{j}}\right) \Rightarrow \sigma_{-}^{\mathrm{j}}\left(\tau_{\mathrm{j}}\right)=\sigma_{+}^{\mathrm{f}}\left(\tau_{\mathrm{j}}\right)+2 \mathrm{~K}_{\mathrm{s}}
$$

Since the sign unity property for the case of 2- and 3-dimension, the distance of the passing point from the shifted-coordinate origins are decreasing by passing:

$$
\left\{\begin{array}{c}
\text { Passing Right }:\left|\sigma_{+}^{j}\left(t_{j}\right)\right|=\left|\sigma_{(}^{f}\left(t_{j}\right)\right|-2 K_{s} \\
\text { Passing Left }:\left|\sigma_{-}^{j}\left(t_{j}\right)\right|=\left|\sigma_{+}^{f}\left(t_{j}\right)\right|-2 K_{s}
\end{array}\right.
$$

Since, Lyapunov-like function is defined in the shifted-coordinate, it shows a "passing jump" between the initial and final functions. Rewrite the Lyapunov-like function in the original coordinate:

$$
\begin{aligned}
V_{s} & =x_{s}^{T} P x_{s} \\
& =\left(x-k_{s} \operatorname{sgn}\left(x_{1}\right)\right)^{T} P\left(x-k_{s} \operatorname{sgn}\left(x_{1}\right)\right) \\
& =x^{T} P x+K_{s}^{T} P K_{s}-2 K_{s}^{T} P x \operatorname{sgn}\left(x_{1}\right)
\end{aligned}
$$



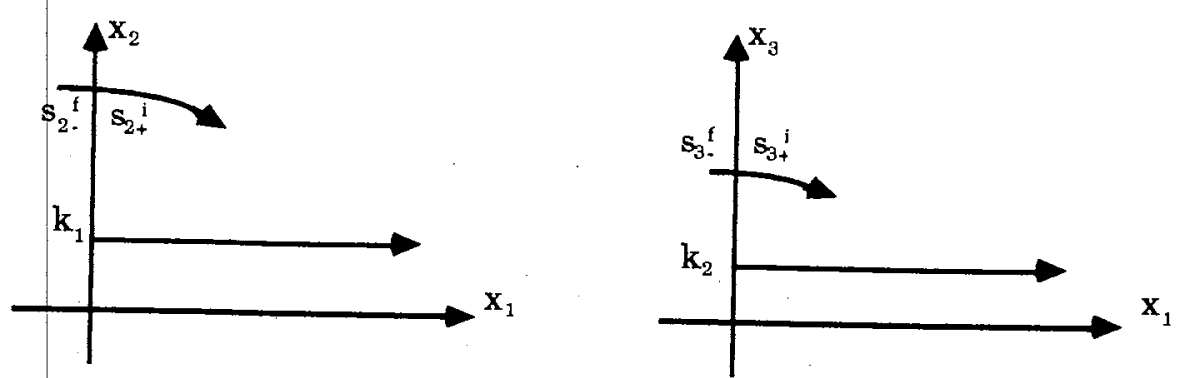

Figure 3.11 A solution point shows passing right through the hyperplane

Passing Jump of Lyapunov-like Function.

i) Passing right case $\left(x_{2}>k_{1}\right)$ : If $x_{2}>k_{1}$, then the solution point passes from the left half space to the right half space, i.e., $\dot{\mathbf{x}}_{1}>0$. The difference of Lyapunov-like function between the passing points is

$$
\begin{aligned}
J\left(\tau_{j}\right)_{\left(x_{2}>k_{1}\right)} & =V_{+}^{\mathrm{i}}\left(\tau_{j}\right)-V_{-}^{\mathrm{f}}\left(\tau_{\mathrm{j}}\right) \\
& =-4 \mathrm{~K}_{\mathrm{s}}^{\mathrm{T}} \mathrm{P} \mathrm{x} \\
& =-4\left[\begin{array}{llll}
0 & \mathrm{k}_{1} & \cdots & \mathrm{k}_{\mathrm{n}-1}
\end{array}\right] \mathrm{P}\left[\begin{array}{c}
0 \\
\mathrm{x}_{2} \\
\vdots \\
\mathrm{x}_{\mathrm{n}}
\end{array}\right]
\end{aligned}
$$

For the third-order system, as shown in Figure 3.11, all of the final passing states are positive except the first state $x_{1} \approx 0$ - (see proof Appendix C.3). Each elements of shifted-coefficient $K_{s}$ is positive except the first one. We can see that the passing jump $J\left(t_{j}\right)$ is a form of bilinear function, $x P y$, and the (n-1) $x(n-1)$ submatrix of $P$ of equation (3.36) is positive definite. The sign unity of the bilinear function do not guarantee that the passing jump $\mathrm{J}$ is strictly negative (see Appendix A.2). With the diagonally dominant submatrix $\mathrm{P}_{\mathrm{n}-1}$ and the sign unity of the bounded states and positive coefficients $k$ 's, the passing jump $J$ is negative. After approximation, the passing 
jump $\mathrm{J}$ is

$$
J=-4\left\{k_{1} P_{22} x_{2}+k_{2} P_{33} x_{3}+\ldots+k_{n-1} P_{n n} x_{n}\right\}
$$

ii) Passing left case: If $x_{2}<-k_{1}$, then the solution point passes from the left half space to the right half space, i.e., $\dot{\mathbf{x}}_{1}<0$. The difference of Lyapunovlike function between the passing points is

$$
\begin{aligned}
J\left(\tau_{j}\right)_{\left(x_{2}<-k_{1}\right)} & =V_{-}^{\mathrm{i}}\left(\tau_{j}\right)-V_{+}^{\mathrm{f}}\left(\tau_{\mathrm{j}}\right) \\
& =4 \mathrm{~K}_{\mathrm{s}}^{\mathrm{T}} \mathrm{P} \mathrm{x} \\
& =4\left[\begin{array}{llll}
0 & \mathrm{k}_{1} & \ldots & \mathrm{k}_{\mathrm{n}-1}
\end{array}\right] \mathrm{P}\left[\begin{array}{c}
0 \\
\mathrm{x}_{2} \\
\vdots \\
\mathrm{x}_{\mathrm{n}}
\end{array}\right]
\end{aligned}
$$

Since each domain in the shifted-coordinate is symmetry about the origin, all of the features are the same each other. In this domain, the sign unity is negative and consequently the passing jump $\mathrm{J}$ is negative. After approximation, the passing jump $J$ is

$$
J=4\left\{k_{1} P_{22} x_{2}+k_{2} P_{33} x_{3}+\ldots+k_{n-1} P_{n n} x_{n}\right\}
$$

where $\mathrm{x}_{\mathrm{i}}$ is negative $(\mathrm{i}=2, \ldots, \mathrm{n})$.

Hence, with a passing condition, the solution point of sliding observer approaches to the shifted-coordinate origins quickly as shown above passing jump.

\subsubsection{Lyapunov-like Stability Theorem}

\subsection{Lyapunov-like Stability Analysis}

The objective is to asymptotically stabilize the system through a series of switching, which is expressed by the set of passing points along with 
sequences of switching timing [Peleties, 1991]:

$$
\left\{\begin{array}{l}
0 \leq \tau_{j}<\infty, \forall \tau_{j} \in \tau_{s}=\tau_{o}, \tau_{1}, \ldots, \tau_{k-1}, \tau_{k}, \ldots \\
\left.s\left(x_{s}, \tau_{j}\right)\right\}=s\left(\tau_{0}\right), s\left(\tau_{1}\right), \ldots, s\left(\tau_{k}\right), \ldots
\end{array}\right.
$$

Lyapunov-like Function Sets on the Hyperplane. Let us define the initial and final Lyapunov-like function set[Peleties, 1991]:

$$
\left\{\mathrm{V}_{\mathrm{s}}\left(\mathrm{x}_{\mathrm{s}}, \tau_{\mathrm{j}}\right)\right\}=\mathrm{V}_{+}^{\mathrm{i}}\left(\tau_{0}\right), \mathrm{V}_{+}^{\mathrm{f}}\left(\tau_{1}\right), \mathrm{V}_{-}^{\mathrm{i}}\left(\tau_{1}\right), \mathrm{V}_{-}^{\mathrm{f}}\left(\tau_{2}\right), \mathrm{V}_{+}^{\mathrm{i}}\left(\tau_{2}\right), \mathrm{V}_{+}^{\mathrm{f}}\left(\tau_{3}\right), \ldots, \mathrm{V}_{\mathrm{s}}^{\mathrm{f}}\left(\tau_{\mathrm{j}}\right)
$$

and the initial and final passing point set:

$$
\left\{\mathrm{s}_{\mathrm{s}}\left(\mathrm{x}_{s}, \tau_{\mathrm{j}}\right)\right\}=\mathrm{s}_{+}^{\mathrm{i}}\left(\tau_{0}\right), \mathrm{s}_{+}^{\mathrm{f}}\left(\tau_{1}\right), \mathrm{s}_{-}^{\mathrm{i}}\left(\tau_{1}\right), \mathrm{s}_{-}^{\mathrm{f}}\left(\tau_{2}\right), \mathrm{s}_{+}^{\mathrm{i}}\left(\tau_{2}\right), \mathrm{s}_{+}^{\mathrm{f}}\left(\tau_{3}\right), \ldots, \mathrm{s}_{\mathrm{s}}^{\mathrm{f}}\left(\tau_{\mathrm{j}}\right)
$$

The sequence of Lyapunov-like functions is identical to that of passing 
points. The detailed notion of Lyapunov-like function sequences is follows:

$$
\begin{aligned}
& \text { Passing Left Passing Right }
\end{aligned}
$$

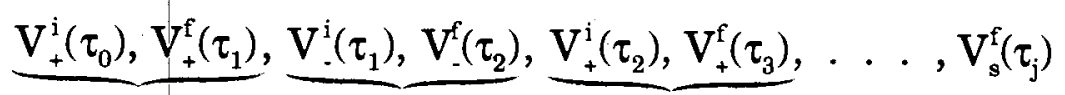

Right Domain Left Domain Right Domain Until satisfying passing condition

The set $\left\{V_{+}\left(x_{s}, \tau_{j}\right)\right\}$ for the right half space can be defined as Lyapunov-like function sequences corresponding to the right passing points sequences $\left\{s_{+}\left(x_{+}, \tau_{j}\right)\right\}$

$$
\begin{aligned}
& \left\{V_{+}\left(x_{+}, \tau_{j}\right)\right\}=V_{+}^{\mathrm{i}}\left(\tau_{0}\right), V_{+}^{\mathrm{f}}\left(\tau_{1}\right), \mathrm{V}_{+}^{\mathrm{i}}\left(\tau_{2}\right), \mathrm{V}_{+}^{\mathrm{f}}\left(\tau_{3}\right), \ldots, \mathrm{V}_{+}^{\mathrm{f}}\left(\tau_{\mathrm{j}}\right) \\
& \left\{\mathrm{s}_{+}\left(\mathrm{x}_{+}, \tau_{\mathrm{j}}\right)\right\}=\mathrm{s}_{+}^{\mathrm{i}}\left(\tau_{0}\right), \mathrm{s}_{+}^{\mathrm{f}}\left(\tau_{1}\right), \mathrm{s}_{+}^{\mathrm{i}}\left(\tau_{2}\right), \mathrm{s}_{+}^{\mathrm{f}}\left(\tau_{3}\right), \ldots, \mathrm{s}_{+}^{\mathrm{f}}\left(\tau_{\mathrm{j}}\right)
\end{aligned}
$$

For the left half space, $\left\{\mathrm{V} .\left(\mathrm{x}_{\mathrm{s}}, \tau_{\mathrm{j}}\right)\right\}$, and $\left\{\mathrm{s}_{-}\left(\mathrm{x}_{-}, \tau_{\mathrm{j}}\right)\right\}$ are

$$
\begin{aligned}
& \left\{\mathrm{V}_{-}\left(\mathrm{x}_{-}, \tau_{\mathrm{j}}\right)\right\}=\mathrm{V}_{-}^{\mathrm{i}}\left(\tau_{1}\right), \mathrm{V}_{-}^{\mathrm{f}}\left(\tau_{2}\right), \mathrm{V}_{-}^{\mathrm{i}}\left(\tau_{3}\right), \mathrm{V}_{-}^{\mathrm{f}}\left(\tau_{4}\right), \ldots, \mathrm{V}_{-}^{\mathrm{f}}\left(\tau_{\mathrm{j}}\right) \\
& \left\{\mathrm{s}_{-}\left(\mathrm{x}_{-}, \tau_{\mathrm{j}}\right)\right\}=\mathrm{s}_{-}^{\mathrm{i}}\left(\tau_{1}\right), \mathrm{s}_{-}^{\mathrm{f}}\left(\tau_{2}\right), \mathrm{s}_{-}^{\mathrm{i}}\left(\tau_{3}\right), \mathrm{s}_{-}^{\mathrm{f}}\left(\tau_{4}\right), \ldots, \mathrm{s}_{-}^{\mathrm{f}}\left(\tau_{\mathrm{j}}\right)
\end{aligned}
$$

Zero-input Responses of the Reaching Dynamics. For the stability of the sliding observer, it is desirable that any initial state asymptotically converge to the origin. Hence, we need to consider the worst case whose initial state is not on the sliding patch and does not satisfy the sliding condition. For this case, by adapting this coordinate transformation, the well-known linear system theory can be applied to interpret the sliding observer as the reaching dynamics which is a linear system in each shifted-coordinate half space.

The reaching dynamics in the shifted-coordinate is 


$$
\dot{x}_{s}=A_{m} x_{s}+\left(\begin{array}{c}
0 \\
\vdots \\
0 \\
u_{d}
\end{array}\right)
$$

where $u_{d}=w-k_{n} \operatorname{sgn}\left(x_{1}\right)$

Let us consider first the internal stability of the reaching dynamics by setting the disturbance input zero, $u_{d}=0$. The internal system is

$$
\dot{x}_{\mathrm{s}}=\mathrm{A}_{\mathrm{m}} \mathbf{x}_{\mathrm{s}}
$$

A candidate Lyapunov-like function for this system:

$$
V_{s}=x_{s}^{T} P x_{s}
$$

Assuming that the Lyapunov-like function is differentiable except switching plane, its derivative with respect to time is

$$
\begin{aligned}
\dot{V}_{s} & =\dot{x}_{s}^{T} P x_{s}+x_{s}^{T} P \dot{x}_{s} \\
& =\left[A_{m} x_{s}\right]_{s}^{T} P x_{s}+x_{s}^{T} P\left[A_{m} x_{s}\right] \\
& =x_{s}^{T} A_{m}^{T} P x_{s}+x_{s}^{T} P A_{m} x_{s}
\end{aligned}
$$

We can see that each reaching dynamics is asymptotically attractive to the shifted origin (or to the sliding patch). If, whenever the solution point passes through the hyperplane, the Lyapunov-like function strictly decreases, then the Lyapunov-like function is strictly decreasing along the trajectory over the whole reaching dynamics domain.

Theorem 3.1 Given an reaching dynamics of the form $\dot{x}_{s}=A_{m} x_{s}$ (where $A_{m}$ : Hurwitz system matrix, $x_{s}=x-K_{s} \operatorname{sgn}\left(x_{1}\right), s=-$ or +$)$, let us consider $a$ quadratic Lyapunov-like function candidate:

$$
V_{s}(x)=x_{s}^{T} P x_{s}, s=-,+
$$


which is a real positive definite on $\mathcal{R}^{\mathrm{n}}$ and unbounded where $\mathrm{V}_{\mathbf{s}}(\mathrm{x})$ is associated with the domain $\Omega_{-}(\mathrm{x}), \Omega_{+}(\mathrm{x})$. Suppose $\left|\mathrm{x}_{2}^{\mathrm{i}}\right|>\mathrm{k}_{1}$ at $\mathrm{x}_{1}=0, \forall \mathrm{x}_{\mathrm{o}} \in \mathbb{R}^{\mathrm{n}}$, there exist constants $\gamma>0, \rho>0$ and a switching sequence

$$
\left\{\mathrm{s}_{\mathrm{s}}\left(\mathrm{x}_{\mathrm{s}}, \tau_{\mathrm{j}}\right)\right\}=\mathrm{s}_{+}^{\mathrm{i}}\left(\tau_{0}\right), \mathrm{s}_{+}^{\mathrm{f}}\left(\tau_{1}\right), \mathrm{s}_{-}^{\mathrm{i}}\left(\tau_{1}\right), \mathrm{s}_{-}^{\mathrm{f}}\left(\tau_{2}\right), \mathrm{s}_{+}^{\mathrm{i}}\left(\tau_{2}\right), \mathrm{s}_{+}^{\mathrm{f}}\left(\tau_{3}\right), \ldots, \mathrm{s}_{\mathrm{s}}^{\mathrm{f}}\left(\tau_{\mathrm{j}}\right), \ldots
$$

as in the followings:

i) In an reaching domain, the total derivative of Lyapunov-like function is negative definite in the domain $\Omega_{s}\left(x_{s}\right)$ [Peleties, 1991]:

$$
\dot{\mathrm{V}}_{\mathrm{s}}\left(\mathrm{x}_{\mathrm{s}}\right) \leq\left.\gamma|| \mathrm{x}_{\mathrm{s}}\right|^{2}<0, \forall \mathrm{x}_{\mathrm{s}} \neq 0, \forall \mathrm{s}, \mathrm{s}=-,+
$$

ii) While the solution point is passing the hyperplane, the Lyapunov-like function always decreases:

$$
V_{s}^{i}\left(\tau_{j}\right)-V_{s}^{\mathrm{f}}\left(\left(\tau_{\mathrm{j}}\right)\right) \leq-\left.\rho|| \sigma_{\mathrm{s}}\left(\tau_{\mathrm{j}}\right)\right|^{2}, \forall \sigma_{\mathrm{s}}\left(\tau_{\mathrm{j}}\right) \in \sigma_{\mathrm{s}}
$$

where the subscripts $\mathrm{s}$ and $\mathrm{s}^{*}$ are: $\operatorname{sign}(\mathrm{s}) \neq \operatorname{sign}\left(\mathrm{s}^{*}\right), \mathrm{s}=-$ or,$+ \mathrm{s}^{*}=+$ or - .

Then, the system is globally asymptotically attractive to the sliding patch that is defined as $\left|x_{2}\right| \leq k_{1}$ at the switching plane $\left(x_{1}=0\right)$.

Proof) The above two conditions mean that the sequence $\left\{V_{s}\left(x_{s}, \tau_{j}\right)\right\}$ strictly decreases along the trajectories up to its limit. This implies that $\mathrm{V}_{\mathrm{s}}^{\mathrm{f}}\left(\sigma\left(\tau_{\mathrm{j}+1}\right)\right)-\mathrm{V}_{\mathrm{s}}^{\mathrm{i}}\left(\sigma\left(\tau_{\mathrm{j}}\right)\right)=\int_{\mathrm{i}^{\mathrm{i}}}^{\mathrm{t}} \dot{\mathrm{V}}\left(\mathrm{x}_{\mathrm{s}}, \tau\right) \mathrm{d} \tau<0$ for each side and $\lim _{\mathrm{j} \rightarrow \infty} \mathrm{V}_{\mathrm{s}}\left(\sigma\left(\tau_{\mathrm{j}}\right)\right)=\mathrm{L} \geq 0$ This limit exists by virtue of the fact that the sequence $\left\{\mathrm{V}_{\mathrm{s}}\left(\sigma\left(\tau_{\mathrm{j}}\right)\right)\right\}$ strictly decreases by the passing condition ii) and lower-bounded by zero:

$$
\begin{aligned}
& \lim _{j \rightarrow \infty} V_{s}^{f}\left(\sigma_{s}\left(\tau_{j+1}\right)\right)-\lim _{j \rightarrow \infty} V_{s}^{i}\left(\sigma_{s}\left(\tau_{j}\right)\right)=L-L=0 \\
= & \lim _{j \rightarrow \infty}\left[V_{s}^{f}\left(\sigma_{s}\left(\tau_{j+1}\right)\right)-V_{s}^{i}\left(\sigma_{s}\left(\tau_{j}\right)\right] \leq \lim _{j \rightarrow \infty}\left[-\gamma|| \sigma_{s}\left(\tau_{j}\right)||^{2}\right] \leq 0 .\right.
\end{aligned}
$$

In other words, $\lim _{j \rightarrow \infty}\left[-\gamma \|\left.\sigma_{\mathrm{s}}\left(\tau_{\mathrm{j}}\right)\right|^{2}\right]=-\gamma \lim _{j \rightarrow \infty}\left[\|\left.\sigma_{\mathrm{s}}\left(\tau_{\mathrm{j}}\right)\right|^{2}\right]=0$, which implies 
$\lim _{j \rightarrow \infty} \sigma_{s}\left(\tau_{j}\right)=0$, which is a sufficient condition for attractiveness to the sliding patch.

函

Example 3.2 Consider following 2-order sliding observer:

$$
\left\{\begin{array}{l}
\dot{x}_{1}=x_{2}-h_{1} x_{1}-k_{1} \operatorname{sgn}\left(x_{1}\right) \\
\dot{x}_{2}=-h_{2} x_{1}-k_{2} \operatorname{sgn}\left(x_{1}\right)+w
\end{array}\right.
$$

where $\mathrm{k}_{1}: 1, \mathrm{k}_{2}: 0, \mathrm{~h}_{1}: 0.1, \mathrm{~h}_{2}: 0.2, \mathrm{w}=0$, initial conditions: $\mathrm{x}_{1}: 0, \mathrm{x}_{2}: 10$

Rewrite the above equation using the shifted-coordinate transformation then we have a reaching dynamics:

where $u_{d}=0$

$$
\left\{\begin{array}{l}
\dot{\mathrm{x}}_{1}=\mathrm{x}_{2 \mathrm{~s}}-\mathrm{h}_{1} \mathrm{x}_{1} \\
\dot{\mathrm{x}}_{2 \mathrm{~s}}=-\mathrm{h}_{2} \mathrm{x}_{1}+\mathrm{u}_{\mathrm{d}}
\end{array}\right.
$$
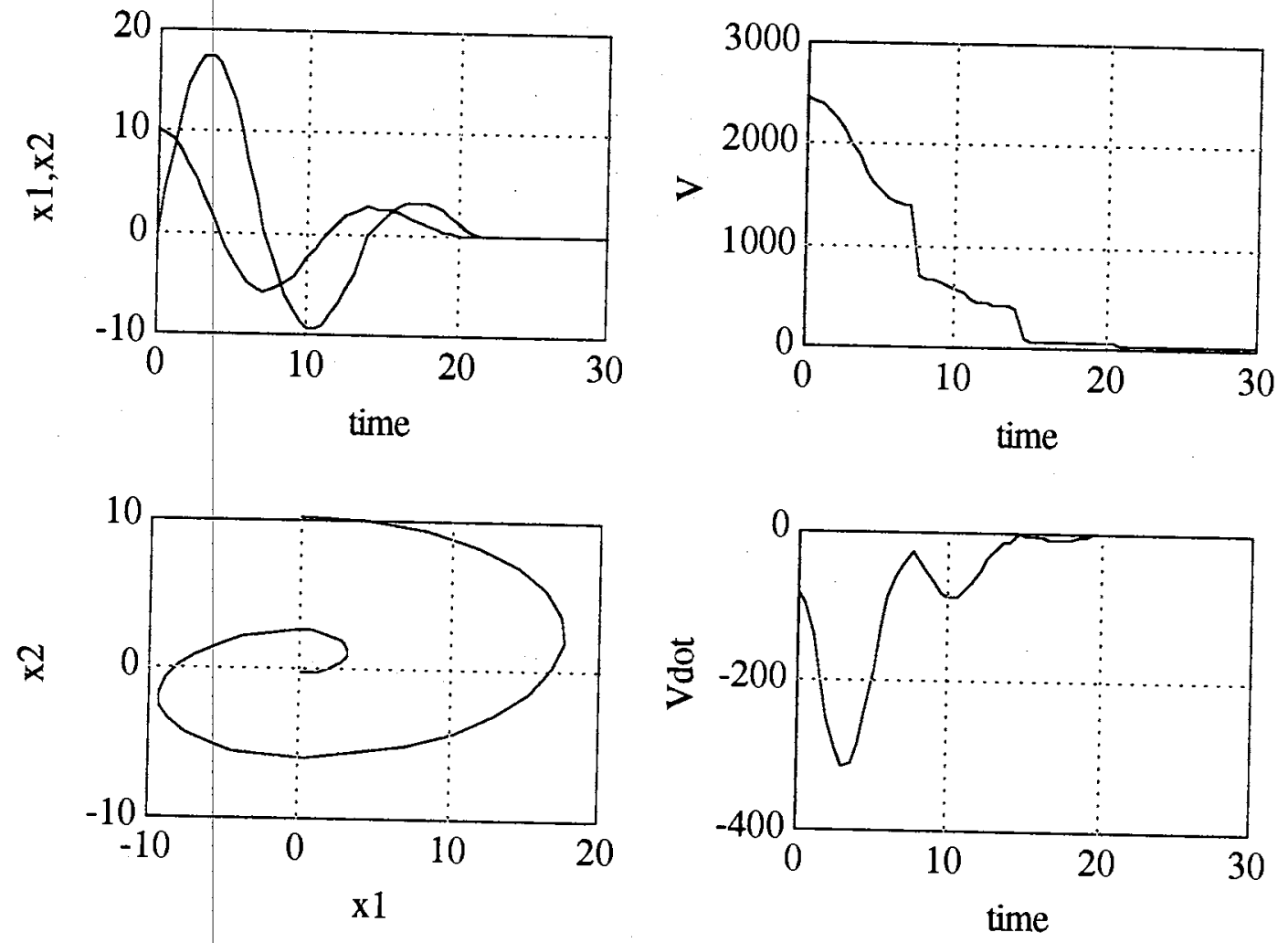

Figure 3.13 Phase plane and Lyapunov-like function 
Lyapunov equation $\mathrm{A}^{*} \mathrm{P}+\mathrm{P}^{*} \mathrm{~A}=\mathrm{Q}$

$$
A_{m}=\left[\begin{array}{ll}
-.1 & 0 \\
-.2 & 0
\end{array}\right], Q=\left[\begin{array}{cc}
-1 & 0 \\
0 & -1
\end{array}\right], P=\left[\begin{array}{cc}
6 & -.5 \\
-.5 & 30.25
\end{array}\right]
$$

The phase plane Figure 3.13 shows that the solution point is asymptotically approaching to the sliding patch via passing the hyperplane $\mathbf{x}_{1}=0$ twice. The Lyapunov-like function shows two passing jumps around 8 and 14 second.

Total Responses of the Reaching Dynamic. Since the disturbance input $u_{d}$ includes the neglected nonlinearities and disturbances, without considering this term, the sliding observer cannot handle uncertain/nonlinear systems. In a linear stability theory, if the internal system of controllable and observable system is stable then the total stability of LTI system is guaranteed . (see Appendix B.1) On the contrary, we cannot conclude the stability of reaching dynamics only based on the zeroinput response of the system.

Assuming that the Lyapunov-like function is differentiable, its derivative with respect to time is

$$
\dot{\mathrm{V}} \mathrm{s}=\dot{\mathrm{x}}_{\mathrm{s}}^{\mathrm{T}} \mathrm{P} \mathrm{x}_{\mathrm{s}}+\mathrm{x}_{\mathrm{s}}^{\mathrm{T}} \mathrm{P} \dot{\mathrm{x}}_{\mathrm{s}}
$$

Plug in the reaching dynamics (3.34) with $u_{d} \neq 0$.

$$
\begin{aligned}
& \dot{\mathrm{V}}_{\mathrm{s}}=\left[\mathrm{A}_{\mathrm{m}} \mathbf{x}_{\mathrm{s}}+\left(\begin{array}{c}
0 \\
\vdots \\
0 \\
\mathrm{u}_{\mathrm{d}}
\end{array}\right]_{\mathrm{s}}^{\mathrm{T}} \mathrm{P} \mathbf{x}_{\mathrm{s}}+\mathbf{x}_{\mathrm{s}}^{\mathrm{T}} \mathrm{P}\left[\mathrm{A}_{\mathrm{m}} \mathbf{x}_{\mathrm{s}}+\left(\begin{array}{c}
0 \\
\vdots \\
0 \\
\mathrm{u}_{\mathrm{d}}
\end{array}\right)\right]\right. \\
& =x_{s}^{T} A_{m}^{T} P x_{s}+x_{s}^{T} P A_{m} x_{s}+2\left\{\begin{array}{c}
0 \\
\vdots \\
0 \\
u_{d}
\end{array}\right\}^{T} P x_{s}
\end{aligned}
$$

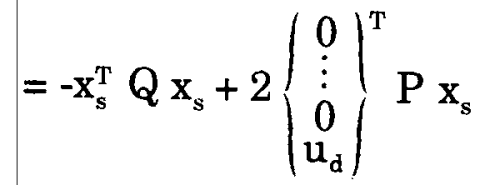


Owing to the 2nd term of right hand side equation, the time derivative of Lyapunov-like function may be not negative definite. At this situation the so-called matehing condition could be a solution that guarantees negative definiteness of $\dot{\mathrm{V}}_{\mathrm{s}}$. However, in real situation, the condition usually cannot be satisfied or it is so conservative that it is almost useless. The every conservativeness comes into the system by sticking to the negative definiteness. Hence it is desirable that the negative definite of $\dot{V}$ in Theorem 3.1 is replaced by strictly decreasing Lyapunov-like function of passing states as following theorem:

Theorem 3.2 Given a reaching dynamics of the form $\dot{x}_{s}=A_{m} x_{s}+u_{d}$ (where $A_{m}$ : Hurwitz system matrix, $x_{s}=x-K_{s} \operatorname{sgn}\left(x_{1}\right)$, $u_{d}$ : input, $s=-$ or + ), let us consider a quadratic Lyapunov-like function candidate:

$$
\mathrm{V}_{\mathrm{s}}(\mathrm{x})=\mathrm{x}_{\mathrm{s}}^{\mathrm{T}} \mathrm{P} \mathrm{x}_{\mathrm{s}}, \mathrm{s}=-,+
$$

which are real positive definite on $R^{\mathrm{n}}$ and unbounded where $V_{s}(x)$ is associated with the domain $\Omega_{-}(\mathrm{x}), \Omega_{+}(\mathrm{x})$. Suppose $\left|\mathrm{x}_{2}^{\mathrm{i}}\right|>\mathrm{k}_{1}$ at $\mathrm{x}_{1}=0, \forall \mathrm{x}_{0} \in \mathbb{R}^{\mathrm{n}}$, there exists constants $\gamma>0, \rho>0$ and a switching sequence $\left\{s_{s}\left(x_{s}, \tau_{j}\right)\right\}$ as in:

i) In an reaching domain, the final Lyapunov-like function $\mathrm{V}_{\mathrm{s}}^{\mathrm{f}}\left(\sigma\left(\tau_{i+1}\right)\right)$ is strictly less than the initial Lyapunov-like function $\mathrm{V}_{\mathrm{s}}^{\mathrm{i}}\left(\sigma\left(\tau_{\mathrm{j}}\right)\right)$ in the domain $\Omega_{\mathrm{s}}\left(\mathrm{x}_{\mathrm{s}}\right)$ :

$$
\mathrm{V}_{\mathrm{s}}^{\mathrm{f}}\left(\sigma\left(\tau_{\mathrm{j}+1}\right)\right)-\mathrm{V}_{\mathrm{s}}^{\mathrm{i}}\left(\sigma\left(\tau_{\mathrm{j}}\right)\right)<-\gamma \|\left.\sigma_{\mathrm{s}}\left(\tau_{\mathrm{j}}\right)\right|^{2}
$$

ii) While the solution point passes the hyperplane, the Lyapunov-like function always decreases:

$$
\left.V_{\mathrm{s}}^{\mathrm{i}}\left(\tau_{\mathrm{j}}\right)-\mathrm{V}_{\mathrm{s}}^{\mathrm{f}} \mathrm{f}\left(\tau_{\mathrm{j}}\right)\right) \leq-\rho \|\left.\sigma_{\mathrm{s}}\left(\tau_{\mathrm{j}}\right)\right|^{2}, \forall \sigma_{\mathrm{s}}\left(\tau_{\mathrm{j}}\right) \in \sigma_{\mathrm{s}}
$$

where the subscripts $\mathrm{s}$ and $\mathrm{s}^{*}$ are: $\operatorname{sign}(\mathrm{s}) \neq \operatorname{sign}\left(\mathrm{s}^{*}\right), \mathrm{s}=-$ or,$+ \mathrm{s}^{*}=+$ or - .

Then, the system is asymptotically attractive to the sliding patch. 
Proof) The proof is the same as Theorem 3.1.

While Theorem 3.1 requires negative definite of $\dot{V}_{s}$, Theorem 3.2 compares the Lyapunov-like function of the initial and the final passing point. In the case of Theorem 3.2, the Lyapunov-like function may not strictly decrease. However, the reaching dynamics is equivalently linear time-invariant system so that the system is bibo stable. The shifted coordinate transformation of the sliding observer enables to apply the linear stability theorem to nonlinear system. All the more, the passing jump brings the shearing effect so that the solution point approaches sliding patch fast.

Example 3.3 Consider the same 2-order sliding observer as that of the former example except the disturbance input:
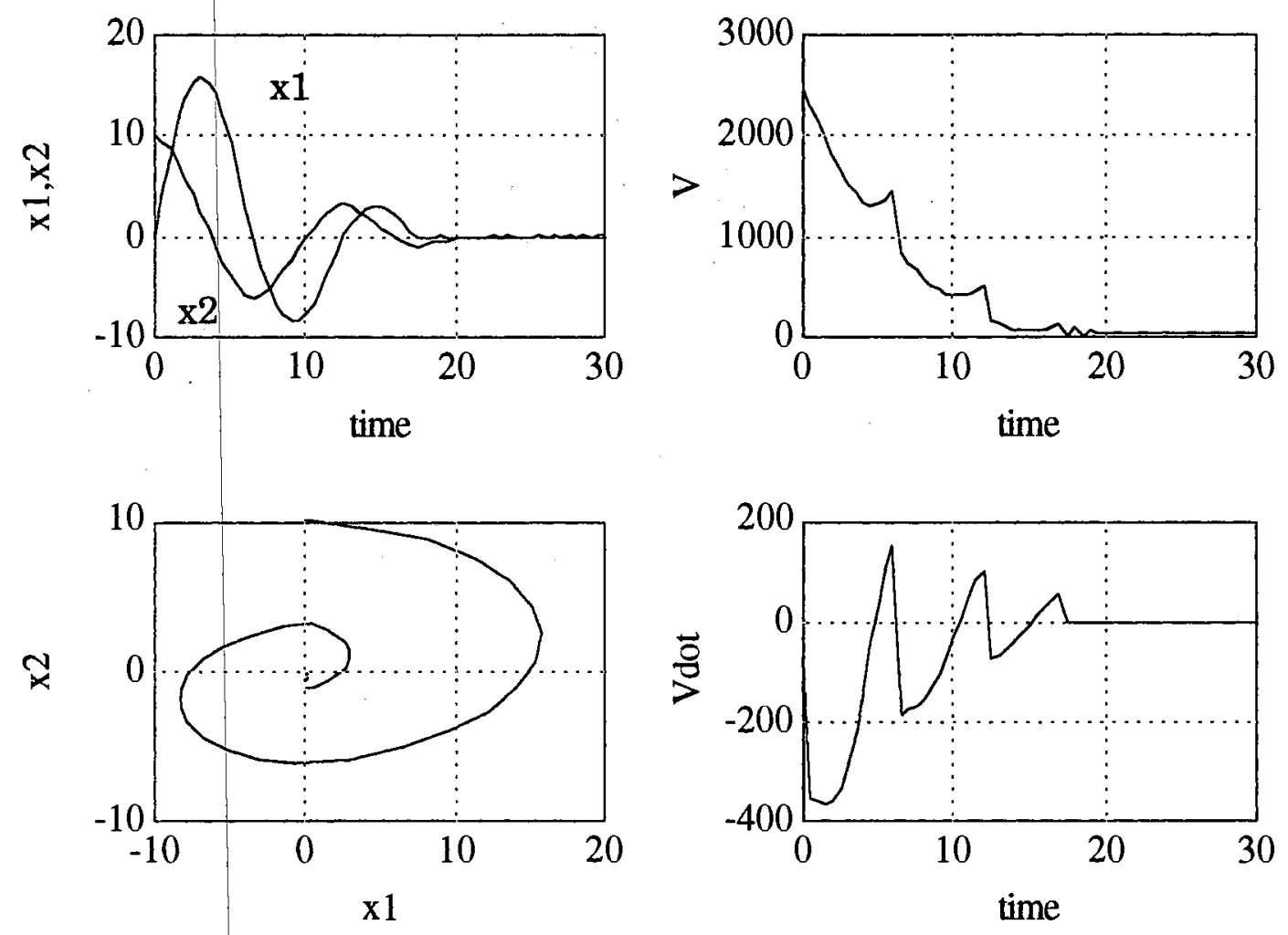

Figure 3.14 Phase plane and Lyapunov-like function 


$$
\left\{\begin{array}{l}
\dot{x}_{1}=x_{2}-h_{1} x_{1}-k_{1} \operatorname{sgn}\left(x_{1}\right) \\
\dot{x}_{2}=-h_{2} x_{1}-k_{2} \operatorname{sgn}\left(x_{1}\right)+w
\end{array}\right.
$$

where $\mathrm{k}_{1}: 1, \mathrm{k}_{2}: 0.5, \mathrm{~h}_{1}: 0.1, \mathrm{~h}_{2}: 0.2, \mathrm{w}=0$, initial conditions: $\mathrm{x}_{1}: 0, \mathrm{x}_{2}: 10$

Rewrite the above equation using the shifted-coordinate transformation then we have a reaching dynamics:

$$
\left\{\begin{array}{l}
\dot{x}_{1}=x_{2 s}-h_{1} x_{1} \\
\dot{x}_{2 s}=-h_{2} x_{1}+u_{d}
\end{array}\right.
$$

where $u_{d}=-0.5^{*} \operatorname{sgn}\left(x_{1}\right)$

The Lyapunov equation is the same as the former one. The phase plane shows that the solution point with disturbance input approaches to the sliding patch faster than the former example because the sign of disturbance input $u_{d}$ is opposite sign of $x_{1}$. The Lyapunov-like function shows several passing jumps around 6.5 second and 12 second and so on. Since, $\dot{V}$ is not negative definite, $\mathrm{V}$ is not strictly decreasing either. However, this example shows the Theorem 3.3 is valid. The passing jump is proved for 2- and 3- order cases in Appendix B. The other requirement, i.e., decreasing sequences of initial and final Lyapunov-like function set, is assured by the design algorithm "SOON"( see Chapter 4).

Finally the next Theorem 3.3 is the generalization of the worst analysis: If the system pseudo-energy decreases as solution trajectory passes from the final point of one domain to the final point of the other domain, then it is possible to stabilize the system.

Theorem 3.3 Given an reaching dynamics of the form $\dot{x}_{s}=A_{m} x_{s}+u_{d}$ (where $A_{m}$ : Hurwitz system matrix, $x_{s}=x-K_{s} \operatorname{sgn}\left(x_{1}\right)$, $u_{d}$ : input, $s=-$ or + ), let us consider a quadratic Lyapunov-like function candidate: 


$$
\mathrm{V}_{\mathrm{s}}(\mathrm{x})=\mathrm{x}_{\mathrm{s}}^{\mathrm{T}} \mathrm{P} \mathrm{x}_{\mathrm{s}}, \mathrm{s}=-,+
$$

which are real positive definite on $\mathscr{R}^{\mathrm{n}}$ and unbounded where $\mathrm{V}_{s}(\mathrm{x})$ is associated with the domain $\Omega_{-}(x), \Omega_{+}(x)$. Suppose $\left|x_{2}^{i}\right|>k_{1}$ at $x_{1}=0, \forall x_{0} \in R^{n}$, there exists a switching sequence $\left\{\sigma_{s}\left(x_{s}, \tau_{j}\right)\right\}$ and there exists constants $\rho>0$ such that the final Lyapunov-like function $\mathrm{V}_{\mathrm{s}^{*}}^{\mathrm{f}}\left(\sigma\left(\tau_{\mathrm{i}+1}\right)\right)$ is strictly less than the precedent final Lyapunov-like function $\left.\mathrm{V}_{\mathrm{s}}^{\mathrm{f}}\left(\sigma \tau_{\mathrm{i}}\right)\right)$ of the domain $\Omega_{\mathrm{s}}\left(\mathrm{x}_{\mathrm{s}}\right)$ :

$$
\mathrm{V}_{\mathrm{s}}^{\mathrm{f}}\left(\sigma\left(\tau_{\mathrm{i}+1}\right)\right)-\mathrm{V}_{\mathrm{s}}^{\mathrm{f}}\left(\sigma\left(\tau_{\mathrm{j}}\right)\right)<-\rho \|\left.\sigma_{\mathrm{s}}\left(\tau_{\mathrm{j}}\right)\right|^{2}
$$

where the subscripts $\mathrm{s}$ and $\mathrm{s}^{*}$ are: $\operatorname{sign}(\mathrm{s}) \neq \operatorname{sign}\left(\mathrm{s}^{*}\right), \mathrm{s}=-$ or,$+ \mathrm{s}^{*}=+$ or - , then the system is asymptotically attractive to the sliding patch.

Proof) The above condition means that the sequence $\left\{\mathrm{V}_{s}^{\mathrm{f}}\left(\mathrm{x}_{\mathrm{s}}, \tau_{\mathrm{j}}\right)\right\}$ strictly decreases along the switching sequence $\left\{\sigma_{s}\left(x_{s}, \tau_{j}\right)\right\}$ up to its limit, i.e., $\lim _{j \rightarrow \infty} \mathrm{V}_{\mathrm{s}}\left(\mathrm{x}\left(\tau_{\mathrm{j}}\right)\right)=\mathrm{L} \geq 0$. This limit exists by virtue of the fact that the sequence $\left\{V_{s}^{f}\left(\sigma\left(\tau_{j}\right)\right)\right\}$ is strictly decreasing and lower-bounded by zero:

$$
\begin{aligned}
& \lim _{j \rightarrow \infty} V_{s^{4}}^{f}\left(\sigma_{s}\left(\tau_{j+1}\right)\right)-\lim _{j \rightarrow \infty} V_{s}^{f}\left(\sigma_{s}\left(\tau_{j}\right)\right)=L-L=0 \\
& =\lim _{j \rightarrow \infty}\left[V_{s^{*}}^{f}\left(\sigma_{s}\left(\tau_{j+1}\right)\right)-V_{s}^{f}\left(\sigma_{s}\left(\tau_{j}\right)\right)\right] \leq \lim _{j \rightarrow \infty}\left[-\rho \| \sigma_{s}\left(\tau_{j}\right)||^{2}\right] \leq 0 .
\end{aligned}
$$

In other words,

$$
\lim _{j \rightarrow \infty}\left[-\rho\left\|\sigma_{s}\left(\tau_{j}\right)\right\|^{2}\right]=-\rho \lim _{j \rightarrow \infty}\left[\left\|\sigma_{s}\left(\tau_{j}\right)\right\|^{2}\right]=0
$$

which implies $\lim _{\mathrm{j} \rightarrow \infty} \sigma_{\mathrm{s}}\left(\tau_{\mathrm{j}}\right)=0$, which is a sufficient condition for attractiveness to the sliding patch.

Since this theorem concerns only the final state sequence $\left\{\mathrm{V}_{s}^{\mathrm{f}}\left(\sigma\left(\tau_{\mathrm{j}}\right)\right)\right\}$, one may worry about the possibilities of growing Lyapunov-like function during 
the reaching dynamics. But the boundedness of Lyapunov-like function is guaranteed by the well-known linear system theory that a Hurwitz linear system is $B I B O$ stable with bounded external input. Since the space is cone about the shifted-coordinate origin, for each reaching domain, decreasing final state sequence $\left\{\mathrm{V}_{\mathrm{s}}^{\mathrm{f}}\left(\sigma\left(\tau_{\mathrm{j}}\right)\right)\right\}$ provides a BIBO stability. Next example shows 3-order case whose eigenvalues are chosen intentionally so small (Figure 3.15) that its solution point approaches slowly to the sliding patch and the attractiveness can be explained by Theorem 3.3 .

Example 3.4 Consider a 3-order sliding observer as that of the former example of Chapter 2 except the disturbance input:

$$
\left\{\begin{array}{l}
\dot{\mathrm{x}}_{1}=\mathrm{x}_{2}-\mathrm{h}_{1} \mathrm{x}_{1}-\mathrm{k}_{1} \operatorname{sgn}\left(\mathrm{x}_{1}\right) \\
\dot{\mathrm{x}}_{2}=\mathrm{x}_{3}-\mathrm{h}_{2} \mathrm{x}_{1}-\mathrm{k}_{2} \operatorname{sgn}\left(\mathrm{x}_{1}\right) \\
\dot{\mathrm{x}}_{3}=-\mathrm{h}_{3} \mathrm{x}_{1}-\mathrm{k}_{3} \operatorname{sgn}\left(\mathrm{x}_{1}\right)+\mathrm{w}
\end{array}\right.
$$

where $\mathrm{K}=\left[\begin{array}{lll}.8 & .31 & .7\end{array}\right]^{\mathrm{T}}, \mathrm{H}=\left[\begin{array}{lll}8 & .31 & .05\end{array}\right]^{\mathrm{T}}$, $\mathrm{w}=0$, initial states: $\mathrm{x}_{1}: 2, \mathrm{x}_{2}:-3, \mathrm{x}_{3}:-6$

The reaching dynamics is:

$$
\left\{\begin{array}{l}
\dot{x}_{1}=x_{2 s}-h_{1} x_{1} \\
\dot{x}_{2 s}=x_{3 s}-h_{2} x_{1} \\
\dot{x}_{3 s}=-h_{3} x_{1}+u_{d}
\end{array}\right.
$$

where $u_{d}=-0.7 * \operatorname{sgn}\left(x_{1}\right)$

Even though the initial state is not on the hyperplane, we can see the sign unity at the first passing. This example shows that after first 3 passings, the final passing Lyapunov-like function is greater than the initial's in each reaching domain until reaching the sliding patch. However, the final Lyapunov-like function sequence is decreasing so that it is attracted to the sliding patch by the Theorem 3.3. 

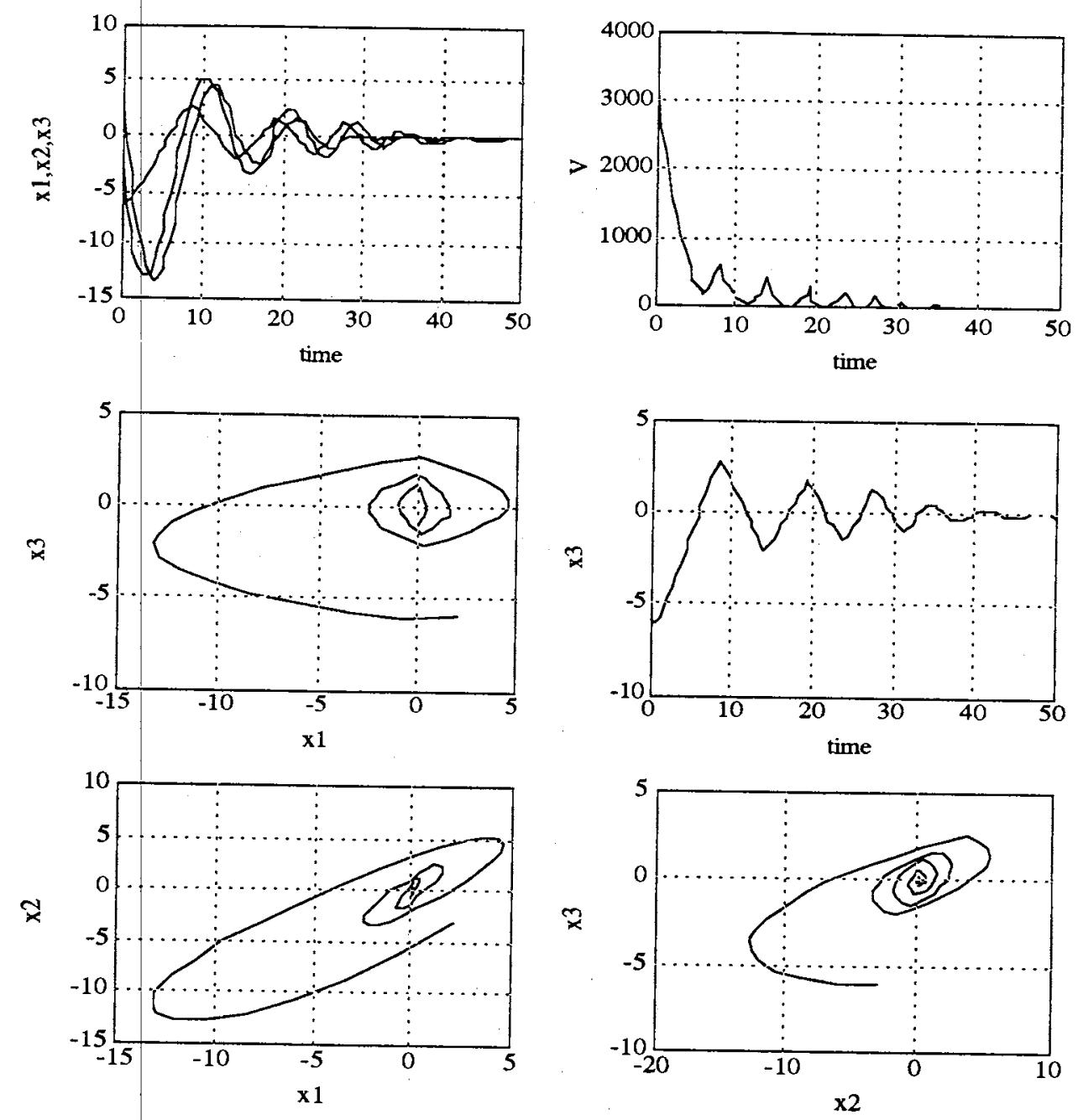

Figure 3.16 Phase plane and Lyapunov-like function

The third mode is the sliding mode that is in the hyperplane $x_{1}=0$ and with the sliding condition $\left|\mathrm{x}_{2}\right|<\mathrm{k}_{1}$.

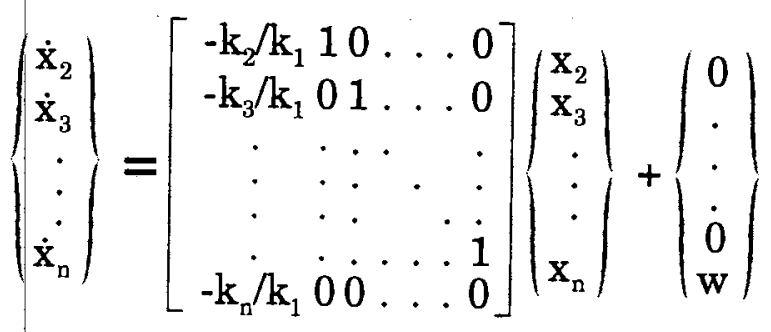


After satisfying the sliding condition, the sliding dynamics is, equivalently, a reduced order linear system. For linear system, asymptotic stability requires that the state of system asymptotically approaches the origin, i.e., $\lim x(t)=0 \forall x\left(\tau_{0}\right) \equiv x_{0}$. For the stability of sliding observer, the $t \rightarrow \infty$

state of system also should approach the origin, via a series of switching. Since the sliding dynamics has switching terms, Lyapunov-like function at the origin may not be zero.

Definition 3.6 A linear dynamical equation is said to be totally stable if and only if for any initial state and for any bounded input the output as well as all the state variables are bounded.

Theorem 3.4 The forced response (zero state) of the sliding equivalent dynamics, i.e., $\dot{x}_{0}=A_{0} x_{0}$, is asymptotically stable if and only if all the eigenvalues of $A_{0}$ have negative real parts.

Proof) Let $P$ be the nonsingular matrix such that $\widehat{A}_{0}=P A_{0} P^{-1}$ and $\widehat{A}_{0}$ is in the Jordan form. In order for the zero state to be asymptotically stable, $\left\|\mathrm{e}^{\mathrm{A}_{0}} t\right\|$ should not only be bounded but also tend to zero $\left(\left\|\mathrm{e}^{\hat{A}_{0}} t\right\| \rightarrow 0\right)$ as $t \rightarrow \infty$. Since every entry of $e^{\hat{A}_{0} t}$ is of the form $t^{k} e^{a_{3} t+w_{j} t}$, we conclude that $\left\|e^{\hat{A}_{0}} t\right\| \rightarrow 0$ as $t \rightarrow \infty$ if and only if all the eigenvalues of $\widehat{A}_{o}$, and consequently of $A_{o}$ have negative real parts.

因

If a linear time-invariant system is asymptotically stable, its zero-input response will approach zero exponentially; thus it is also said to be exponentially stable. It is clear that total stability implies BIBO stability.

\subsubsection{Contour of Lyapunov-like Function}

Consider a contour of equivalent Lyapunov-like function on the 
hyperplane for the previous 3-order instance of Example 3.4. The $2 \times 2$ submatrix is $P_{n-1}=\left[\begin{array}{cc}5 & -.5 \\ -.5 & 73.5\end{array}\right]$.
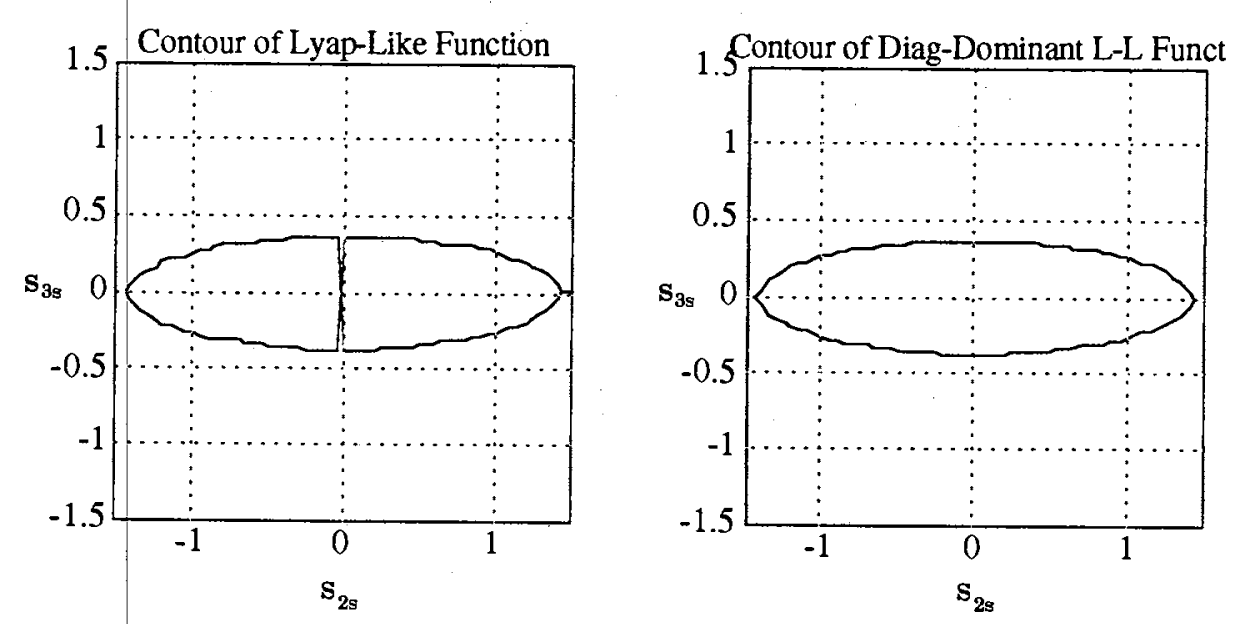

Figure 3.17 Contour of Lyapunov-like functions

The Lyapunov-like function is $V_{+}=5 \sigma_{2+}^{2}-\sigma_{2+} \sigma_{3+}+73.5 \sigma_{3+}^{2}$ whose contour for $\mathrm{V}_{+}=10$ is shown at Figure 3.17(a). The contour of Lyapunov-like function with neglected off-diagonal terms is plotted at Figure 3.17(b). Since the $2 \times 2$ submatrix is diagonal dominant, these are almost identical as expected. For the diagonal dominant case, the principal radius are approximately the square root of corresponding diagonal terms.

$$
R_{\beta_{j}} \cong \sqrt{P_{j j}}, \quad(j=2, \ldots, n)
$$

Consider a initial passing point $\sigma_{2+}>0, \sigma_{3_{+}}>0$ which is on the arc $\mathrm{a}+\mathrm{b}+$ in the hyperplane.

Consider a case of $\dot{\mathrm{V}}_{\mathrm{s}}<0$ or $\mathrm{V}_{\mathrm{s}}^{\mathrm{f}}-\mathrm{V}_{\mathrm{s}}^{\mathrm{i}}<0$, the final point in Figure 3.18 will 
remain inside the ellipsoid $a_{+} b_{+} c_{+} d_{+}$and the sign unity confines the region as $0_{+} c_{+} d_{+}$. If $0>\sigma_{2+}>-2 k_{1}$, then it satisfies the sliding condition. Otherwise, it will pass again the hyperplane and the Lyapunov-like function $V_{s}$ decreases by the jump difference.

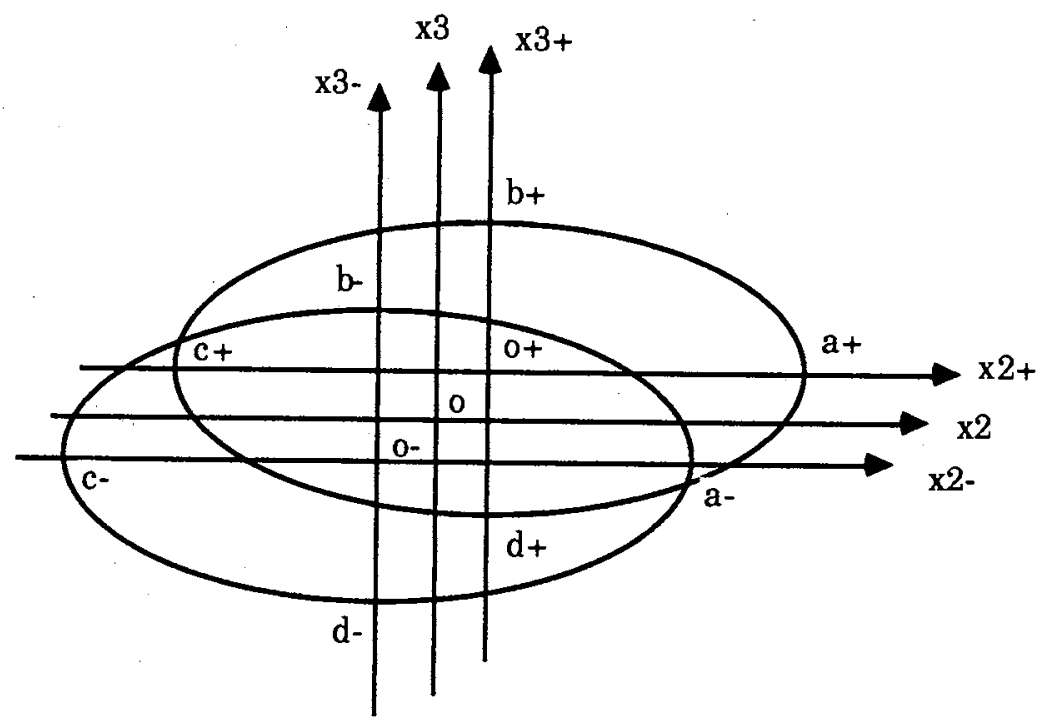

Figure 3.18 Contour of Lyapunov-like function on the hyperplane

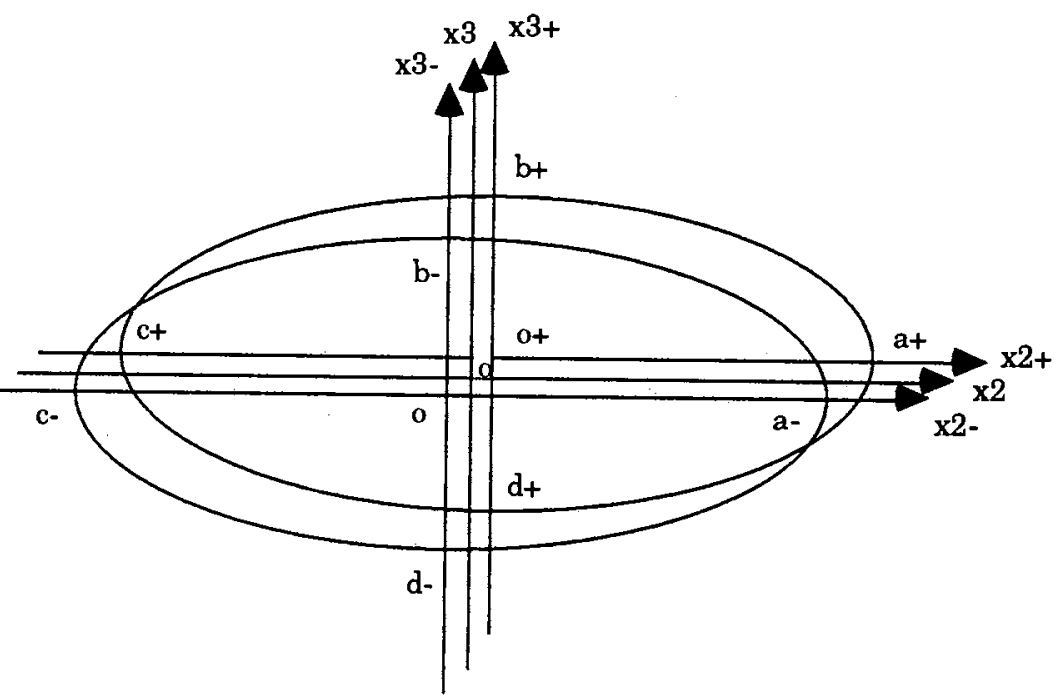

Figure 3.19 Contour of large Lyapunov-like function case 


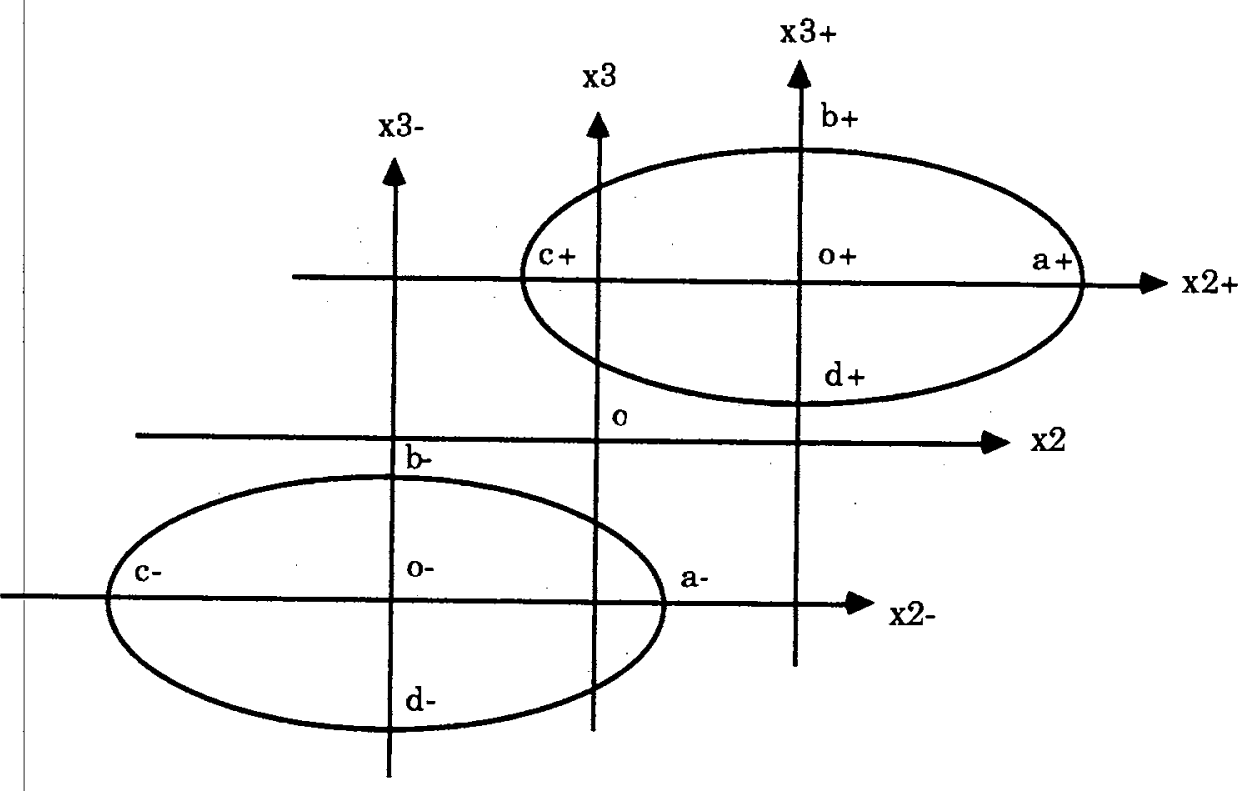

Figure 3.20 Contour of small Lyapunov-like function case

Consider contours of large Lyapunov-like function case as shown in Figure 3.19. It shows that $\mathrm{V}_{+}$covers more region of $\mathrm{V}$. than the previous one as shown in Figure 3.18.

For the case of small Lyapunov-like function, all the final point on the $\operatorname{arc} 0_{+} c_{+} d_{+}$satisfies the sliding condition as shown in Figure 3.20

The ellipsoidal contour is determined by the positive definite matrix $P$. Figure 3.20 shows that a contour does not cover the other side contour at all. This case can increase the Lyapunov-like function by switching. Hence the coefficient $\mathrm{K}$ which determines shifted-coordinate system should be a function of aspect of contour, i.e., principal radius.

The Lyapunov-like function in Figure 3.22 strictly decreases by switching. Hence, for 3-order case, the suggested design methodology of coefficient $K$ is

$$
\frac{\sqrt{\mathrm{P}_{22}}}{\sqrt{\mathrm{P}_{33}}} \approx \frac{\mathrm{k}_{1}}{\mathrm{k}_{2}}
$$




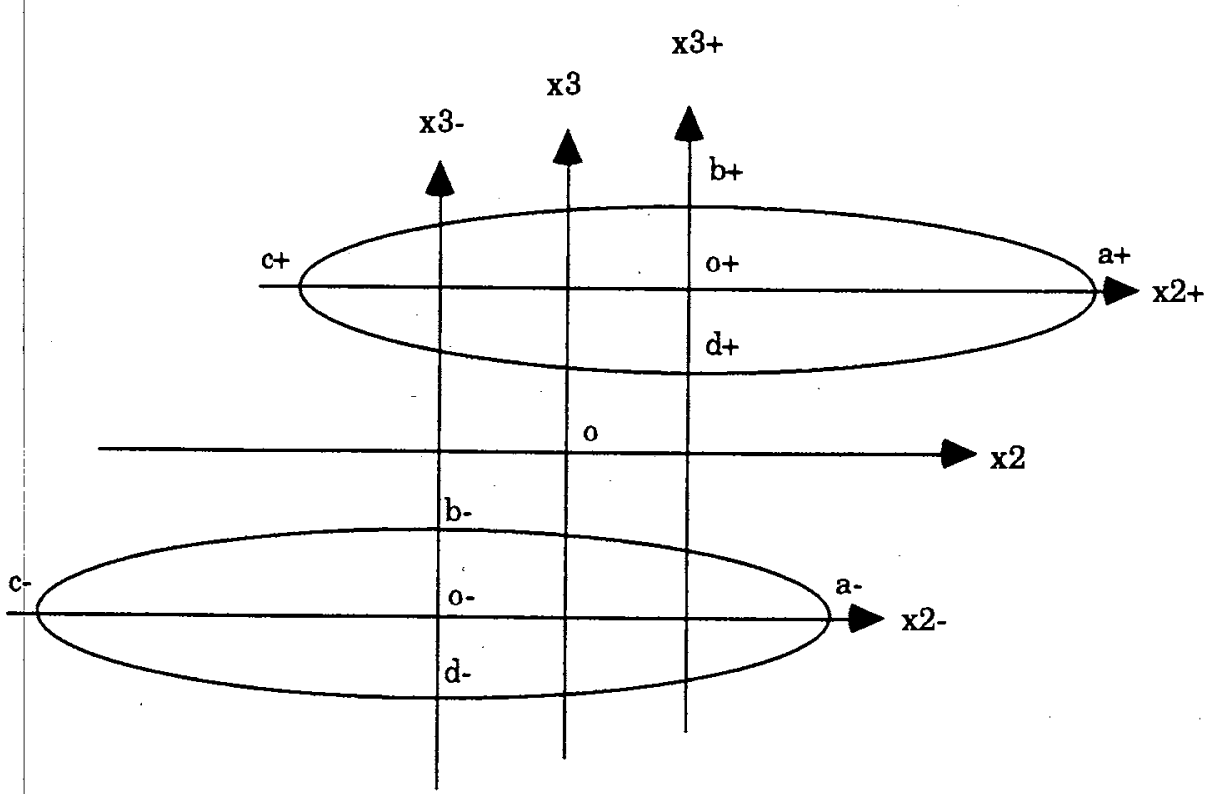

Figure 3.21 Contour of badly designed case

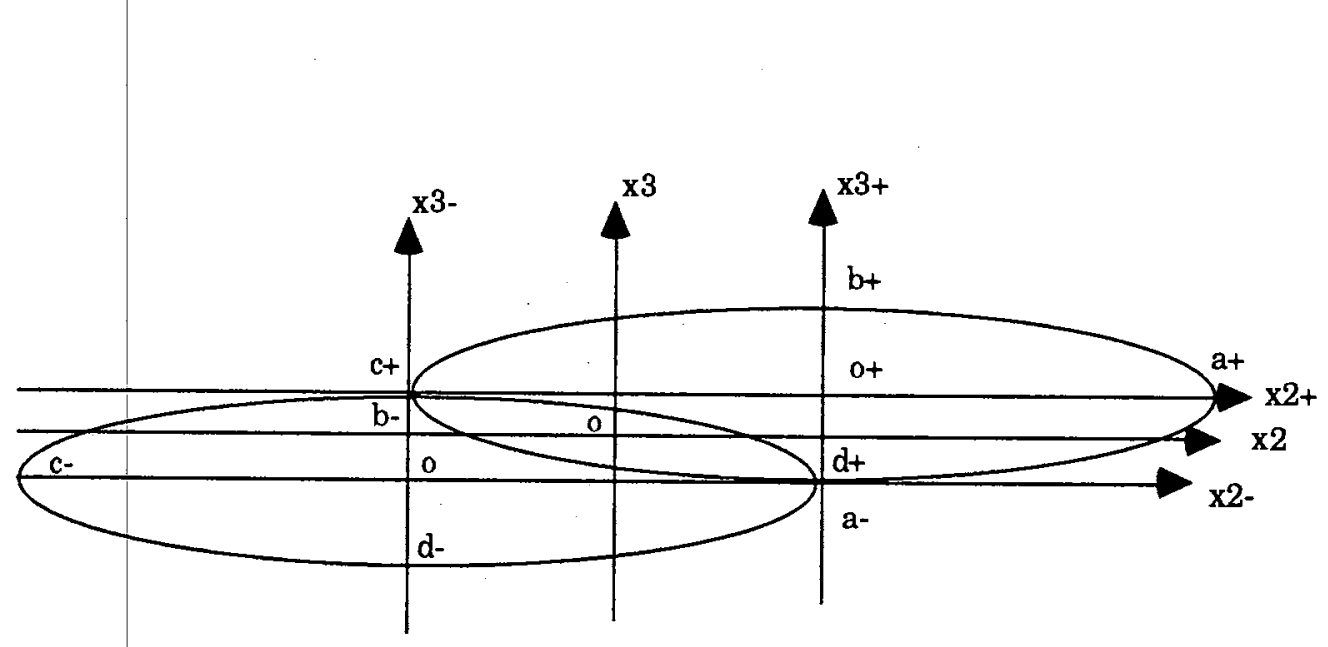

Figure 3.22 Contour of suggested case

Remarks. In Chapter 3.3.3, the general shearing effect was explained by the passing jump of the Lyapunov-like function at the hyperplane. For 3-order system, the sign equalization is prooved in Appendix C.3 and the passing jump $\mathrm{J}$ is always negative. For the 3-order case, the contour of Lyapunov-like function of the passing point not only explains the general 
shearing effect but also suggests how to choose the design coefficient $K$ in relation with the linear coefficient $H$. The sign unification in n-dimensional space was studied in Appendix C but it is not proved analytically yet. Therefore, the design algorithm based on the Lyapunov-like stability Theorem 3.3 are proposed as a future study. 


\section{CHAPTER IV \\ DESIGN OF SLIDING OBSERVERS}

\subsection{Development of Design Algorithm}

\subsubsection{Reaching Dynamics Response}

In Chapter 2, it was showed that the dynamics of the sliding observer in each reaching domain is the same as linear system with the shiftedcoordinate. At the passing instant, even though the velocity field is discontinuous, the trajectory of solution point in reaching domain is continuous. Consequently the dynamical characteristic for the half domain can be extended to the whole domain directly. Hence let us review reaching dynamics in the view point of linear system theory [Chen, 1970] as follows:

$$
\quad x(t)=\underbrace{e^{A(t-\tau)} x(\tau)}_{\text {Zero-input response }}+\underbrace{\int_{\tau}^{t} e^{A(t-\lambda)} B u(\lambda) d \lambda}_{\text {zero-state response }}
$$

A very important property of any linear system is that the responses of the system can be decomposed into two parts, as follows [Chen, 1970]:

Responses due to $\left\{x\left(t_{0}\right), u\left(t_{\omega} \infty\right)\right\}$

$=$ responses due to $\left\{\mathrm{x}\left(\mathrm{t}_{0}\right), 0\right\}+$ responses due to $\left\{0, \mathrm{u}\left(\mathrm{t}_{\mathrm{o}}, \infty\right)\right\}$

The responses due to $\left\{x\left(t_{0}\right), 0\right\}$ are called zero-input responses or transient term: they are generated exclusively by the nonzero initial state $x\left(t_{0}\right)$. The 
responses due to $\left\{0, \mathrm{u}\left(\mathrm{t}_{0}, \infty\right)\right\}$ are called zero-state response or forced term: they are excited exclusively by the input $u\left(t_{o}, \infty\right)$. Hence, for linear systems, we can consider the zero-input responses and zero-state responses independently.

A 2-order reaching dynamics in the shifted-coordinate is [Zhu, 1992]

$$
\left(\begin{array}{l}
\dot{\mathrm{x}}_{1} \\
\dot{\mathrm{x}}_{2}
\end{array}\right)=\left[\begin{array}{ll}
-\mathrm{h}_{1} & 1 \\
-\mathrm{h}_{2} & 0
\end{array}\right]\left(\begin{array}{l}
\mathrm{x}_{1} \\
\mathrm{x}_{2}
\end{array}\right)+\left(\begin{array}{l}
0 \\
\mathrm{u}
\end{array}\right)
$$

where $u=w-k_{2} \operatorname{sgn}\left(x_{1}\right)<0$ for $x_{1}>0$

$$
\left\{\begin{array}{l}
x_{1}=\frac{.1}{s^{2}+h_{1} s+h_{2}} u \\
x_{2}=\frac{s+h_{1}}{s^{2}+h_{1} s+h_{2}} u
\end{array}\right.
$$

With zero initial values, the steady state is obtained by applying the final value theorem. For critical damping, e:g., $\mathrm{h}_{1}{ }^{2}=4 \mathrm{~h}_{2}$, the states by the forcing term are bounded:

$$
\begin{aligned}
& \left|x_{1}\right|=\left|\frac{1}{\left.\mid s+\frac{h_{1}}{2}\right)^{2}} u\right| \\
& \leq \frac{4}{h_{1}^{2}} \max _{0 \leq t t_{f}}|u| \leq \frac{8 k_{2}}{h_{1}^{2}} \\
& \left|x_{2}\right|=\left|\left[\frac{1}{\left(s+\frac{h_{1}}{2}\right)^{2}}+\frac{h_{1} / 2}{\left(s+\frac{h_{1}}{2}\right)^{2}}\right] u\right| \\
& \leq \frac{4}{h_{1}} \max _{0 \leq t<t_{f}}|u| \leq \frac{8 k_{2}}{h_{1}}
\end{aligned}
$$

These bounds are only due to the forcing term. The transient term by the initial conditions cannot be considered in an analytic manner except for 
some special cases. The reaching dynamics is valid only to the next passing point, i.e., $x_{1}=0$. Usually, the steady states bounds are too conservative as compared to the actual passing state values. Even though these bounds are big enough, it is hard to verify that the transient overshoot can be considered in the same manner. The Kalman-Yakubovich lemma is an usual approach to this problem. With the so-called "matching condition", the Lyapunov function of this kind of system is perfect in mathematics but too conservative to find the real applications. The conservativeness is mainly due to the strict negative definiteness of $\dot{\mathrm{V}}$. This conservativeness can be overcome by considering the system's properties, i.e., "shearing effect" etc. Hence, a design algorithm "Sliding Observer design by the wOrst reaching dynamics for Nonlinear/uncertain system" (SOON) is developed to consider all the phenomena--that is the overshoot by initial conditions, the transient by forcing term and shifted-coordinate.

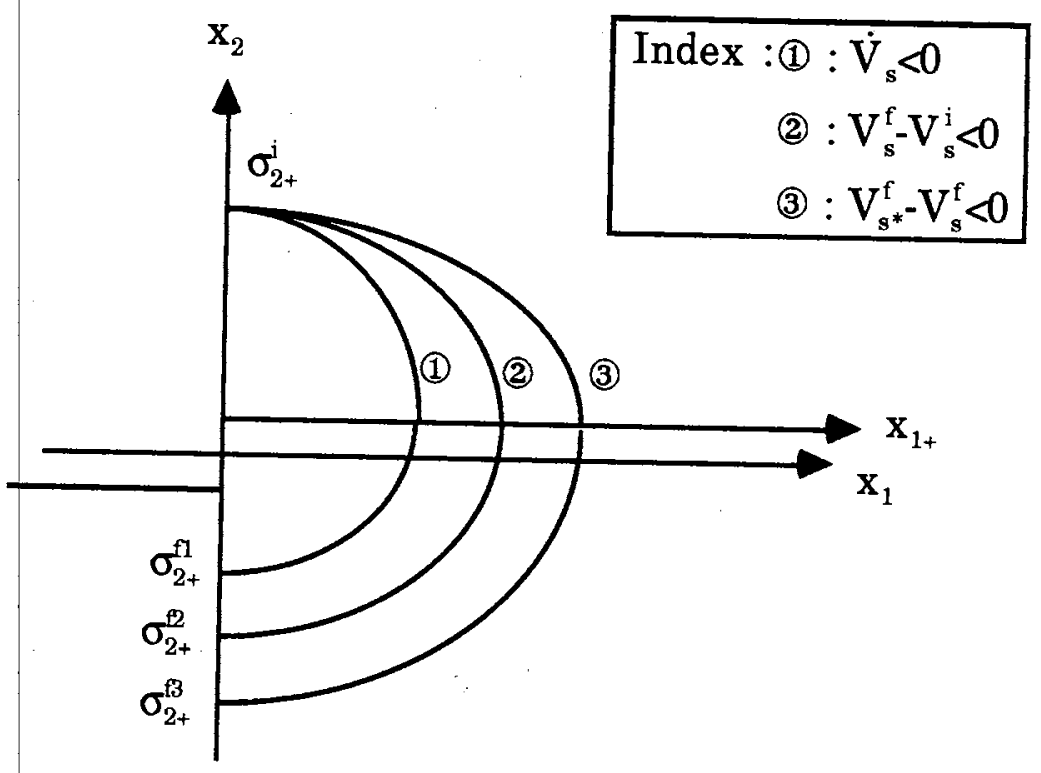

Figure 4.1 Conceptual comparison of stability theorems 


\subsubsection{Conceptual Comparisons of Stability Theorems}

Let us compare the sliding observer stability theorems in Chapter 3 for the case of 2-order reaching dynamics.

2-order Case. Theorem 3.1 requires $\dot{\mathrm{V}}_{\mathrm{s}}<0$. The Lyapunov-like function strictly decreases along the line (1) in Figure 4.1 so that $\sigma_{2+}^{\mathrm{fl}}>-\sigma_{2+}^{\mathrm{i}}\left(\mathrm{s}_{2+}^{\mathrm{f}}>-\mathrm{s}_{2+}^{\mathrm{i}}+2 \mathrm{k}_{1}\right)$

Along the line (2) the Lyapunov-like function $\mathrm{V}_{\mathrm{s}}$ of the final point strictly less than that of initial point: $\mathrm{V}_{\mathrm{s}}^{\mathrm{f}}-\mathrm{V}_{\mathrm{s}}^{\mathrm{i}}<0, \sigma_{2+}^{2}>-\sigma_{2+}^{\mathrm{i}}\left(\mathrm{s}_{2+}^{\mathbb{Q}}>-\mathrm{s}_{2+}^{\mathrm{i}}+2 \mathrm{k}_{1}\right)$

Theorem 3.3 requires $\mathrm{V}^{\mathrm{f}}-\mathrm{V}^{\mathrm{i}}<0\left(\mathrm{~V}\right.$ is the same quadratic function as $\mathrm{V}_{\mathrm{s}}$ except that it is expressed in the original coordinate) The Lyapunov-like function $\mathrm{V}$ of the final point strictly less than that of initial point, i.e., line (3) : $\sigma_{2+}^{\mathfrak{f 3}}>-\sigma_{2+}^{\mathrm{i}}-2 k_{1}\left(\mathbf{s}_{2+}^{\mathrm{f3}}>-\mathbf{s}_{2+}^{\mathrm{i}}\right)$. This condition is exactly the same as the worst case analysis.

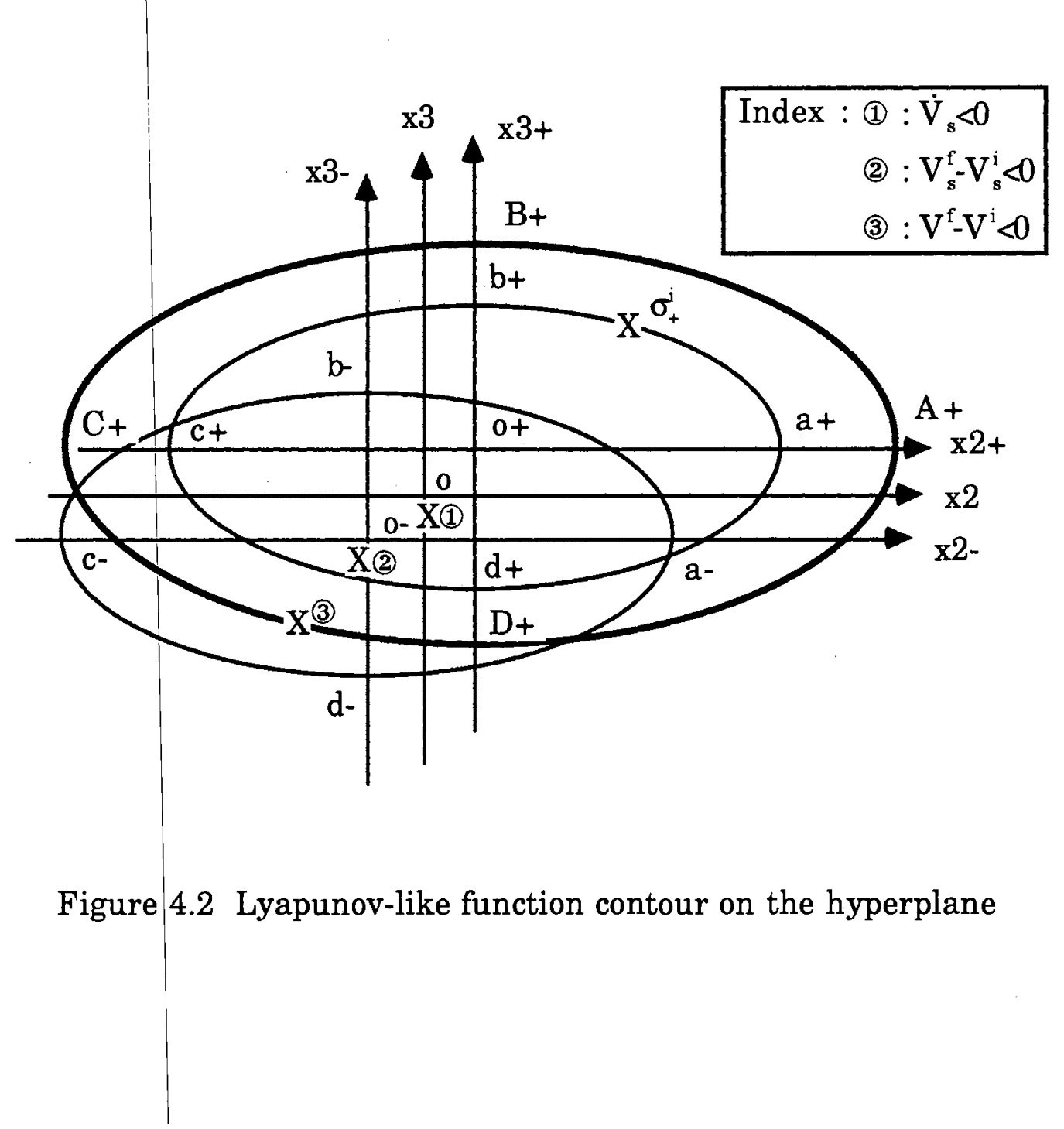


3-order Case. Let us compare the sliding observer stability theorems in Chapter 3 for the case of 3 -order reaching dynamics. Figure 4.2 is only a conceptual diagram to compare the theorems.

The ellipse $a_{+} b_{+} c_{+} d_{+}$is the Lyapunov-like function contour of initial passing point $\sigma_{+}^{i}$. If $\dot{V}_{s}<0$ then a final passing point will fall inside of the ellipse $a_{+} b_{+} c_{+} d_{+}$. If the final passing point $X(1)$ is $-2 k_{1}<\sigma_{2+}^{f}<0$ then it satisfies the sliding condition. If a final passing point, e.g. X(2), is inside the ellipse $a_{+} b_{+} c_{+} d_{+}$then $V_{s}^{f}-V_{s}^{i}<0$. Hence the stability of this case is guaranteed by Theorem 3.2. If a final passing point is the point $\mathrm{X}$ (3) then its contour $A_{+} B_{+} C_{+} D_{+}$is larger than the contour $a_{+} b_{+} c_{+} d_{+}$. However, the Lyapunov-like function of the sequenced initial passing point $\sigma^{i}$ is less than the initial passing point $\sigma_{+}^{+}$. In Figure 4.2 the ellipse a.b.c.d. encloses the final passing point X(3) .

\subsubsection{Sliding Observer Design Algorithm}

It is hard to obtain an analytic solution of the forced response of reaching dynamics except some special cases. The analytic bounds of the forcing term like equations (4.5) and (4.6) are not only conservative but also hard to get for higher order system. The transient term by the initial conditions cannot be considered in an analytic manner also. Since the Lyapunov-like stability Theorem 3.2 and Theorem 3.3 require the Lyapunov-like function of the final passing point only, it is reasonable to obtain the worst bound of final passing point via a simulation. According to the observer requirements, the design criteria can be adjusted iteratively. However, the goal of the design procedure is to make a stable observer. Hence, the first design procedure is suggested to make the initially bounded error dynamics converge 
to the sliding patch directly as follows:

Design Procedures for Direct Converging to the Sliding Patch. (SOON)

1. Determine the system order

2. Determine the maximum bound of the disturbance $w$

3. Determine the bound of the initial states

4. Choose proper linear correction coefficients

- According to the desirable converging speed

5. Simulate SIMNON program Reaching I

- Worst reaching dynamics

6. Designing the switching constant $\mathrm{K}$

- The switching constant $\mathrm{k}_{\mathrm{n}-1}$ is determined by half Max $\sigma_{\mathrm{n}}^{\mathrm{f}}$

- For convergence purpose only, the coefficient $\mathrm{k}_{\mathrm{n}-1}$ does not need to be half of $\operatorname{Max} \sigma_{n}^{f}$

- For $n_{-}$th order case, the suggested coefficient $\mathrm{K}$ is

$$
\frac{\sqrt{\mathrm{P}_{22}}}{\sqrt{\mathrm{P}_{33}}} \approx \frac{\mathrm{k}_{1}}{\mathrm{k}_{2}}, \frac{\sqrt{\mathrm{P}_{33}}}{\sqrt{\mathrm{P}_{44}}} \approx \frac{\mathrm{k}_{2}}{\mathrm{k}_{3}}, \ldots, \frac{\sqrt{\mathrm{P}_{\mathrm{n}-1}}}{\sqrt{\mathrm{P}_{\mathrm{n}}}} \approx \frac{\mathrm{k}_{\mathrm{n}}}{\mathrm{k}_{\mathrm{n}-1}}
$$

where $P_{\mathrm{ii}}$ is obtained from the Lyapunov equation with the proper matrix $Q$.

7. Tuning the design constants:

- If the switching constant k's are big compared to the desired precisions then $h_{j}$ 's are need to be increased

- The characteristic equation (2.24) of the sliding equivalent dynamics:

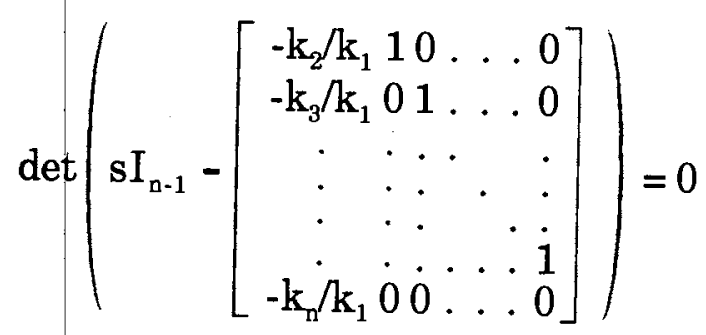

- Steady state error equation (2.54) in sliding region 
Remark. If the characteristic equation has some positive eigen value, then the sliding observer error dynamics will form a "limit" cycle near the sliding patch. Equation (4.6) is based on the contour analysis (3.73). The linear correction coefficient $\mathrm{H}$ is also related to the matrix $\mathrm{Q}$ and $\mathrm{P}$ by the Lyapunov equation (3.58). However, for the second and the third order system, (4.6) has only one term or not. Hence, the tuning procedure between the design constant $\mathrm{H}$ and $\mathrm{K}$ is actually important. For more general design procedure based on the Lyapunov-like stability theorem is suggested as a future study.

\subsection{Comparative Example with VSS Observer}

As a comparative example for the sliding observer and VSS observer, consider a nonlinear system as follows:

$$
\begin{aligned}
& \dot{\mathrm{z}}_{1}=\mathrm{z}_{2} \\
& \dot{\mathrm{z}}_{2}=\mathrm{g}(\mathrm{z}, \mathrm{w})
\end{aligned}
$$

where $\mathrm{g}(\mathrm{z}, \mathrm{w})=-\sin \left(\mathrm{z}_{1}\right)$

\subsubsection{Sliding Observer}

For this system, the sliding observer can be written:

$$
\begin{aligned}
& \dot{\mathrm{z}}_{1}=\widehat{\mathrm{z}}_{2}+\mathrm{h}_{1} \mathrm{x}_{1}+\mathrm{k}_{1} \mathrm{x}_{1} \\
& \hat{\mathrm{z}}_{2}=\hat{\mathrm{g}}(\mathrm{z}, \mathrm{w})+\mathrm{h}_{2} \mathrm{x}_{1}+\mathrm{k}_{2} \mathrm{x}_{1}
\end{aligned}
$$

where $\widehat{\mathrm{g}}(\mathrm{z}, \mathrm{w})=-\sin \left(\widehat{\mathrm{z}}_{1}\right)$

For the sliding observer, it needs only one measurement case and the output equations are

$$
\begin{aligned}
& \mathrm{y}_{1}=\mathrm{z}_{1} \\
& \mathrm{y}=\widehat{\mathrm{z}}_{1}
\end{aligned}
$$


The sliding observer error dynamics is

$$
\left\{\begin{array}{l}
\dot{x}_{1}=x_{2}-h_{1} x_{1}-k_{1} \operatorname{sgn}\left(x_{1}\right) \\
\dot{x}_{2}=-h_{2} x_{1}-k_{2} \operatorname{sgn}\left(x_{1}\right)+u_{d}
\end{array}\right.
$$

where $\mathrm{x}_{1}=\mathrm{z}_{1}-\widehat{\mathbf{z}}_{1}, \mathrm{x}_{2}=\mathrm{z}_{2}-\widehat{\mathrm{z}}_{2}, \mathrm{u}_{\mathrm{d}}=\mathrm{g}(\mathrm{z}, \mathrm{w})-\hat{\mathrm{g}}(\widehat{\mathrm{z}}, \mathrm{w})$ and $\hat{\mathrm{g}}(\widehat{\mathrm{z}}, \mathrm{w})=-\sin \left(\widehat{\mathrm{z}}_{1}\right)$.

The numerical data for the system are

$$
x_{1}^{i}=0.57, x_{2}^{i}=-1 ; \text { initial error states }
$$

The linear correction coefficients choosed as $h_{1}=2$ and $h_{2}=1.2$ to place poles around -1 and to make the system underdamped. With the given bound of initial state, the bound of disturbance input is evaluated as $u_{d}=1$. Hence $\mathrm{k}_{2}=1$ may be a reasonable starting value. For the design algorithm SOON, the initial states are assumed as $x_{1}^{i}=0, x_{2}^{i}=1.5$. The simulated results are shown in Figure 4.3. The worst final passing point is $\sigma_{2+}^{f}=-0.95$ whose absolute value is less the initial value. According to Theorem 3.2, this system is stable. If the shifting coordinate constant $\mathrm{k}_{1}$ is assigned as half of the worst final passing point, then it will make the error state converge to sliding patch directly. Finally the tested design constant are

$$
\mathrm{k}_{1}=0.5, \mathrm{k}_{2}=1, \mathrm{~h}_{1}=2, \mathrm{~h}_{2}=1.2
$$

The simulation results of the sliding observer as shown in Figure 4.4 is compared with the VSS (Variable Structure Systems) observer which is designed by [Walcott et al.]. 


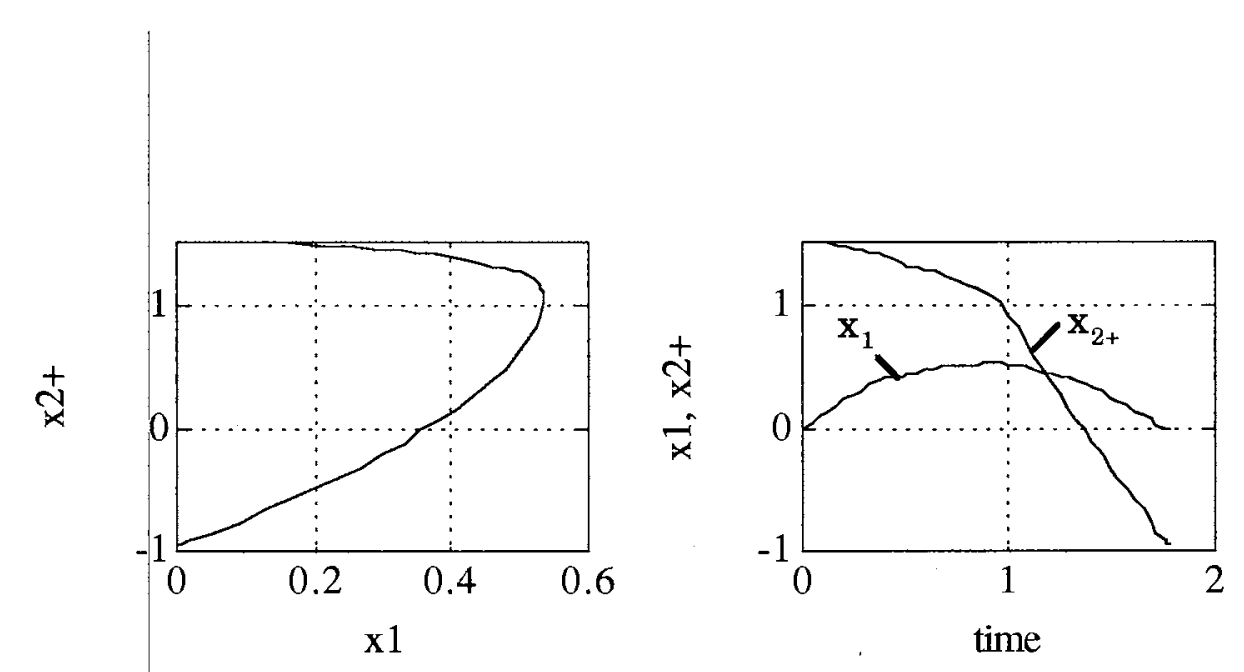

Figure 4.3 Simulation results of SOON
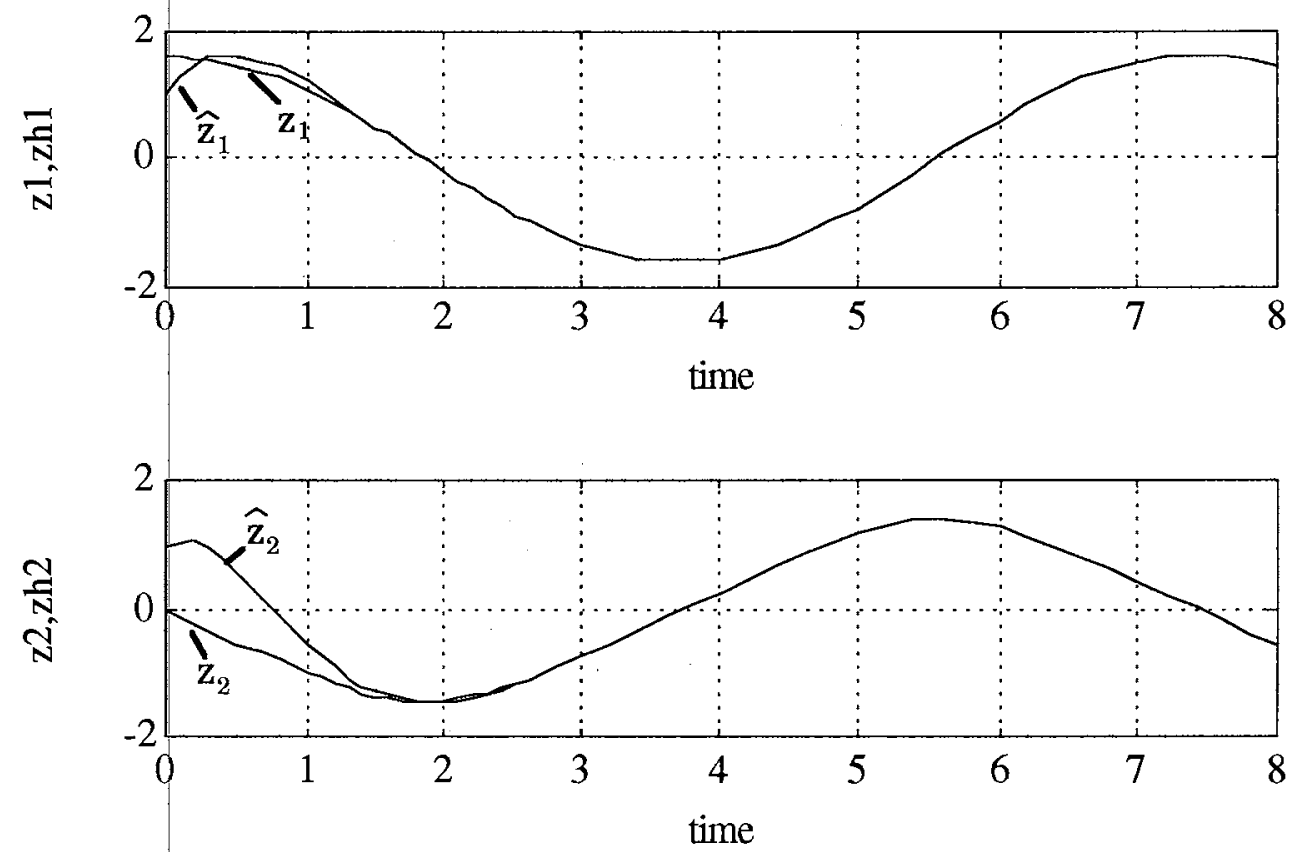

Figure 4.4 Sliding observer simulation results

\subsubsection{VSS Observer}

The suggested VSS observer (see detail [Walcott, 1987]) is

$$
\begin{aligned}
& \dot{\hat{\mathrm{z}}}_{1}=-\widehat{\mathrm{z}}_{1} \\
& \hat{\mathrm{z}}_{2}=-\widehat{\mathrm{z}}_{1}-\widehat{\mathrm{z}}_{2}+\widehat{\mathrm{g}}(\widehat{\mathrm{z}}, \mathrm{w})
\end{aligned}
$$

where $\hat{g}(\widehat{z}, w)=-2 \operatorname{sgn}\left(\widehat{z}_{1}+\widehat{z}_{2}-y_{2}\right)$ 
To design VSS observer, so-called matching condition should be satisfied. It has the form as follows:

$$
\mathrm{P} B=\mathrm{C}^{\mathrm{T}}
$$

where $\mathrm{P}$ is the unique positive-definite solution to the Lyapunov equation.

To satisfy this condition two measurement are necessary and the output equation is

$$
\mathrm{y}_{2}=\mathrm{z}_{1}+\mathrm{z}_{2}
$$

For the same initial condition as previous one, the VSS observer is simulated.

Compared to VSS observer, the sliding observer can be designed without satisfying the so-called matching condition as shown above. Another superiority in favor of the sliding observer design methodology is that, according to design requirements, its characteristic may be easily adjusted by tuning the design coefficient $\mathrm{H}$ and $\mathrm{K}$.

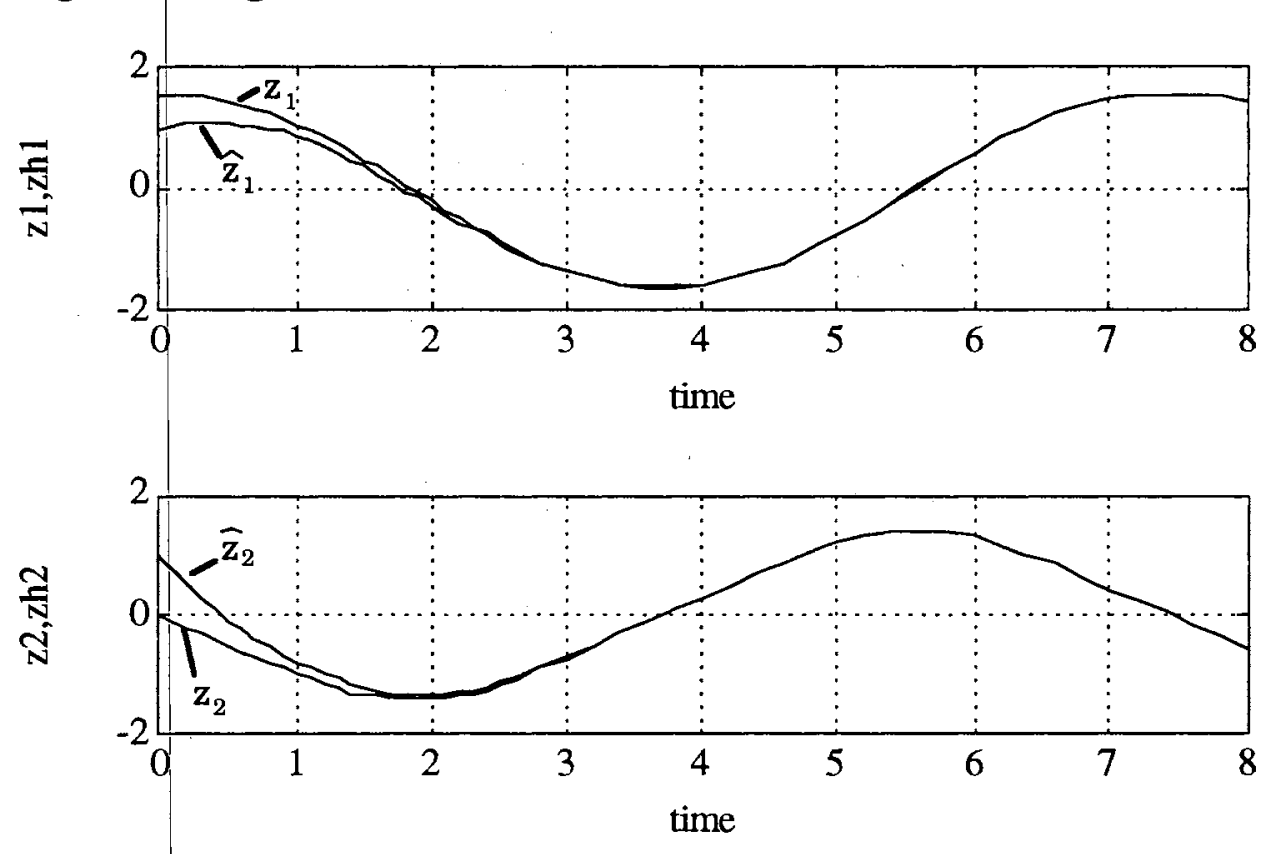

Figure 4.5 VSS observer simulation results 


\subsection{Inverted pendulum with a moving support}

Since Stephenson [Stephenson, 1908] predicted the possibility of inverting the unstable equilibrium of a pendulum on an end by applying a vertical periodic force at the bottom, parametric excitations of an inverted pendulum have been studied by many investigators [Nayfeh, 1979]. Sethna [Sethna, 1973] proved that an inverted pendulum can be stable near the upright position for arbitrary vertical excitations with the condition that they are fast and the time average of the square of the velocity of the vertical excitations is greater than the square of the time average of the velocity of these motions by a constant that depends on the system parameters.

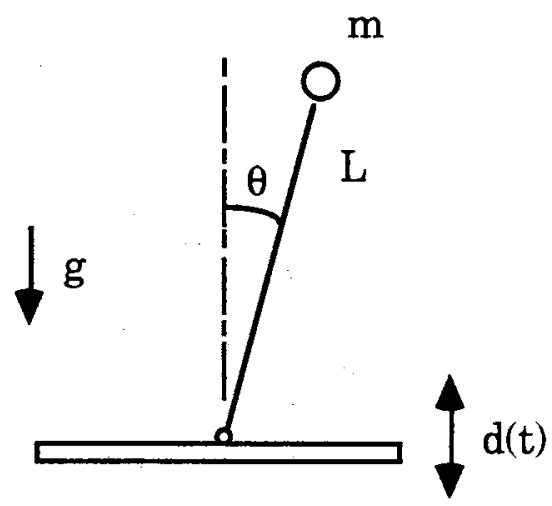

Figure 4.6 Inverted pendulum with a moving support

The equation of motion is derived as follows:

$$
\ddot{\theta}+\frac{g}{L} \sin (\theta)=\frac{1}{L}(\cos (\theta) \dot{\theta} \dot{d}+\sin (\theta) \ddot{d})
$$

where $g=9.8 \mathrm{~m} / \mathrm{sec}^{2}, \mathrm{~L}=0.5 \mathrm{~m}, \mathrm{~d}(\mathrm{t})=0.001 * \sin (100 \mathrm{t})$

Rewrite equation (1) in the state space form: 


$$
\left\{\begin{array}{l}
\dot{z}_{1}=z_{2} \\
\dot{z}_{2}=-\frac{g}{L} \sin \left(z_{1}\right)+\frac{\dot{d}}{L} \cos \left(z_{1}\right) z_{2}+\frac{\dot{d}}{L} \sin \left(z_{1}\right)
\end{array}\right.
$$

The system model for the sliding observer design is

$$
\left\{\begin{array}{l}
\dot{\mathrm{z}}_{1}=\mathrm{z}_{2} \\
\dot{\mathrm{z}}_{2}=\mathrm{w}
\end{array}\right.
$$

where $\mathrm{w}$ is the neglected nonlinearities and model uncertainties.

Let us assume each initial state is bounded by 0.01 then the disturbance input bound is obtained as follows:

$$
\begin{aligned}
& |w| \leq\left|-\frac{\mathrm{g}}{\mathrm{L}} \sin \left(\mathrm{z}_{1}\right)\right|+\left|\frac{\dot{\mathrm{d}}}{\mathrm{L}} \cos \left(\mathrm{z}_{1}\right) \mathrm{z}_{2}\right|+\left|\frac{\ddot{\mathrm{d}}}{\mathrm{L}} \sin \left(\mathrm{z}_{1}\right)\right| \\
& \left|-\frac{\mathrm{g}}{\mathrm{L}} \sin \left(\mathrm{z}_{1}\right)\right| \leq 0.2,\left|\frac{\dot{\mathrm{d}}}{\mathrm{L}} \cos \left(\mathrm{z}_{1}\right) \mathrm{z}_{2}\right| \leq 0.01,\left|\frac{\ddot{\mathrm{d}}}{\mathrm{L}} \sin \left(\mathrm{z}_{1}\right)\right| \leq 0.2
\end{aligned}
$$

The suggested sliding observer is

$$
\left\{\begin{array}{l}
\dot{\mathrm{z}}_{1}=\widehat{\mathrm{z}}_{2}+\mathrm{h}_{1} \mathrm{x}_{1}+\mathrm{k}_{1} \operatorname{sign}\left(\mathrm{x}_{1}\right) \\
\dot{\mathrm{z}}_{2}=\widehat{\mathrm{w}}+\mathrm{h}_{2} \mathrm{x}_{1}+\mathrm{k}_{2} \operatorname{sign}\left(\mathrm{x}_{1}\right)
\end{array}\right.
$$

The linear correction coefficients were chosen as $h_{1}=10$ and $h_{2}=30$ to place poles around -5 and to make the system underdamped. Simply assign the function $\widehat{w}=0$ to the sliding observer. When the bound of initial state is known as 0.01 , the bound of disturbance input is evaluated as $u_{d}=0.5$. Hence $\mathrm{k}_{2}=0.5$ may be a reasonable starting value. With the data, the design algorithm SOON was simulated and the simulated results are as follows: 

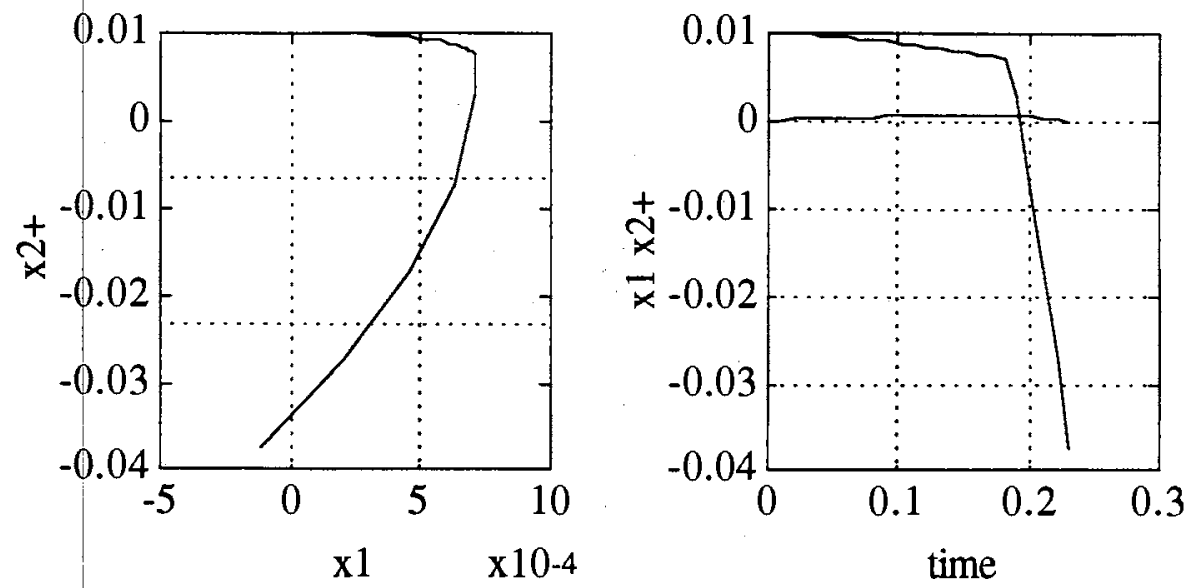

Figure 4.7 Simulation results of SOON

For the design algorithm SOON, the initial states are assumed as $x_{1}^{i}=0, x_{2}^{i}=0.01$. The simulated results are shown in Figure 4.7. The worst final passing point is $\sigma_{2+}^{f}=-0.035$ whose absolute value is greater the initial value. If the shifted coordinate constant $\mathrm{k}_{1}$ is assigned as half of the worst final passing point, then it will make the error state converge to sliding patch directly according to Theorem 3.3. Since the neglected nonlinearities are sinusoidal functions only, the constant $k_{1}$ can be chosen small as according to the desired observing accuracy. Finally the tested design constants are

$$
\mathrm{k}_{1}=0.005, \mathrm{k}_{2}=0.5, \mathrm{~h}_{1}=10, \mathrm{~h}_{2}=30
$$

Even though the initial error of the simulation is as big as twice of the initial error of the design algorithm, the simulation shows that the solution point converges to sliding patch directly as shown in Figure 4.8. This is not a surprising result because the design algorithm by worst case is conservative as expected. 
(a) Time domain plot of the states

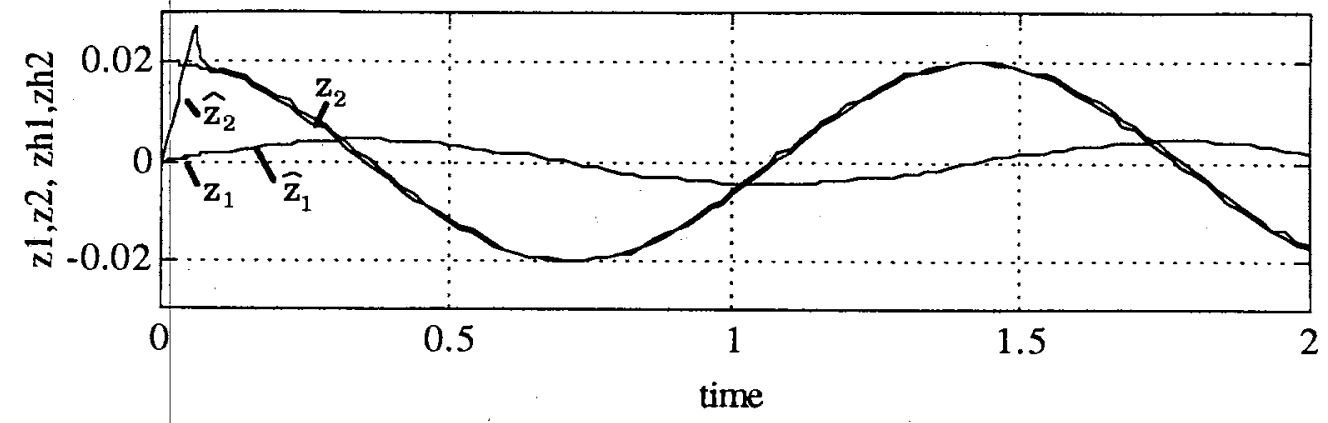

(b) Phase plane
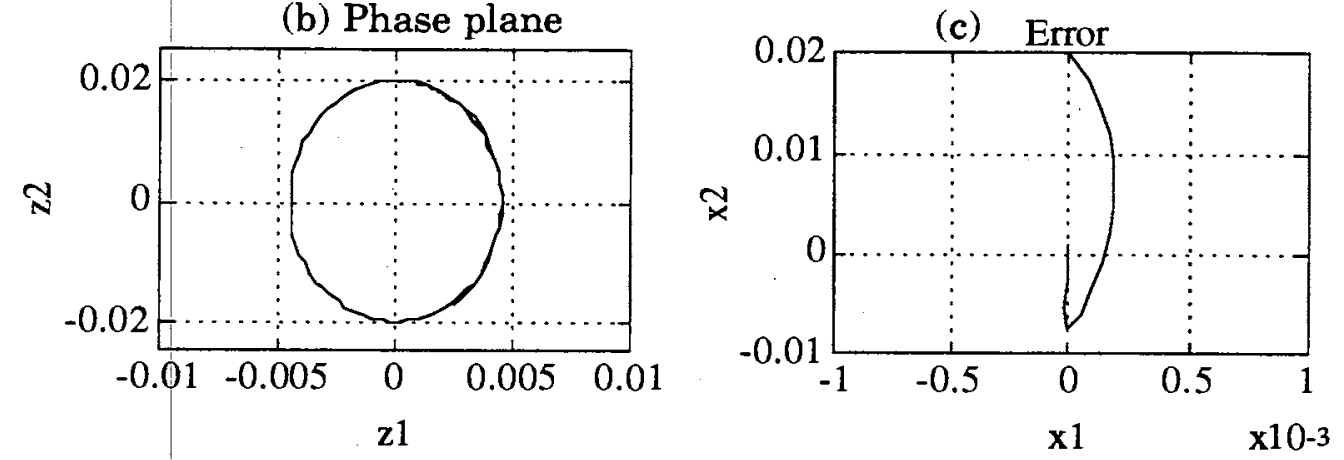

Figure 4.8 Sliding observer simulation results

(a) Time domain plot of the actual and estimated states

(b) Phase plane of the actual states

(c) Phase plane of the error states of the sliding observer

Hence, if the nonlinear model of system is exactly known and if it is composed with sinusoidal functions whose means are zero, then the smaller constant $\mathrm{k}_{1}$ can be chosen safely.

\subsection{Nonlinear Mass-Spring System with Friction}

Consider a 2-order nonlinear system, consisting of a mass connected to a nonlinear spring in the presence of dry friction and stiction, in canonical form: 
where $g(z, w)=-\kappa z_{1}^{3}-f_{s}\left(z_{2}\right)+w, k$ is a constant nonlinear spring coefficient, and $f_{s}\left(z_{2}\right)$ represents dry friction with stiction.

For this system, the sliding mode observer can be written:

$$
\begin{aligned}
& \hat{\mathrm{z}}_{1}=\widehat{\mathrm{z}}_{2}+\mathrm{h}_{1} \mathrm{x}_{1}+\mathrm{k}_{1} \mathrm{x}_{1} \\
& \hat{\mathrm{z}}_{2}=\widehat{\mathrm{g}}(\mathrm{z}, \mathrm{w})+\mathrm{h}_{2} \mathrm{x}_{1}+\mathrm{k}_{2} \mathrm{x}_{1} \\
& \mathrm{y}=\widehat{\mathrm{z}}_{1}
\end{aligned}
$$

The sliding observer error dynamics is

$$
\left\{\begin{array}{l}
\dot{x}_{1}=x_{2}-h_{1} x_{1}-k_{1} \operatorname{sgn}\left(x_{1}\right) \\
\dot{x}_{2}=-h_{2} x_{1}-k_{2} \operatorname{sgn}\left(x_{1}\right)+u_{d}
\end{array}\right.
$$

where $\mathrm{x}_{1}=\mathrm{z}_{1}-\widehat{\mathrm{z}}_{1}, \mathrm{x}_{2}=\mathrm{z}_{2}-\widehat{\mathrm{z}}_{2}, \mathrm{u}_{\mathrm{d}}=\mathrm{g}(\mathrm{z}, \mathrm{w})-\hat{\mathrm{g}}(\widehat{\mathrm{z}}, \mathrm{w})$.

The numerical data for the system are

$$
\begin{aligned}
& \kappa=1.0, F_{s}=1.0 ; \text { static friction, } F_{d}=0.75 ; \text { dynamic friction } \\
& x_{1}^{i}=0, x_{2}^{i}=0.5 ; \text { initial values }
\end{aligned}
$$

The linear correction coefficients was chosen as $h_{1}=8$ and $h_{2}=16$ according to pole placement of linear system theory. Simply assign the function $\widehat{g}(z, w)=0$. With the given bound of initial state, the bound of disturbance input is evaluated as $u_{d}=2$. Hence $k_{2}=2$ may be a reasonable starting value.

With the data simulate the design algorithm SOON and the results are as follows:

The worst final passing point is $\sigma_{2+}^{f}=-0.44$ whose absolute value is less the initial value. According to Theorem 3.2, this system is stable. Hence, we can freely assign the coordinate shifting constant $k_{1}$. Consider the 
desirable accuracy $\mathrm{x}_{1}$, take $\mathrm{k}_{1}=0.01$. Finally the tested design constant are

$$
\mathrm{k}_{1}=0.01, \mathrm{k}_{2}=2, \mathrm{~h}_{1}=8, \mathrm{~h}_{2}=16
$$

The simulation results by SOON is compared with the sliding observer which is designed by the absolute stability theorem and whose switching function is saturation functions instead of signum function (see Appendix B.2). The numerical data for the saturation sliding observer is the same as new sliding observer except the design coefficients:

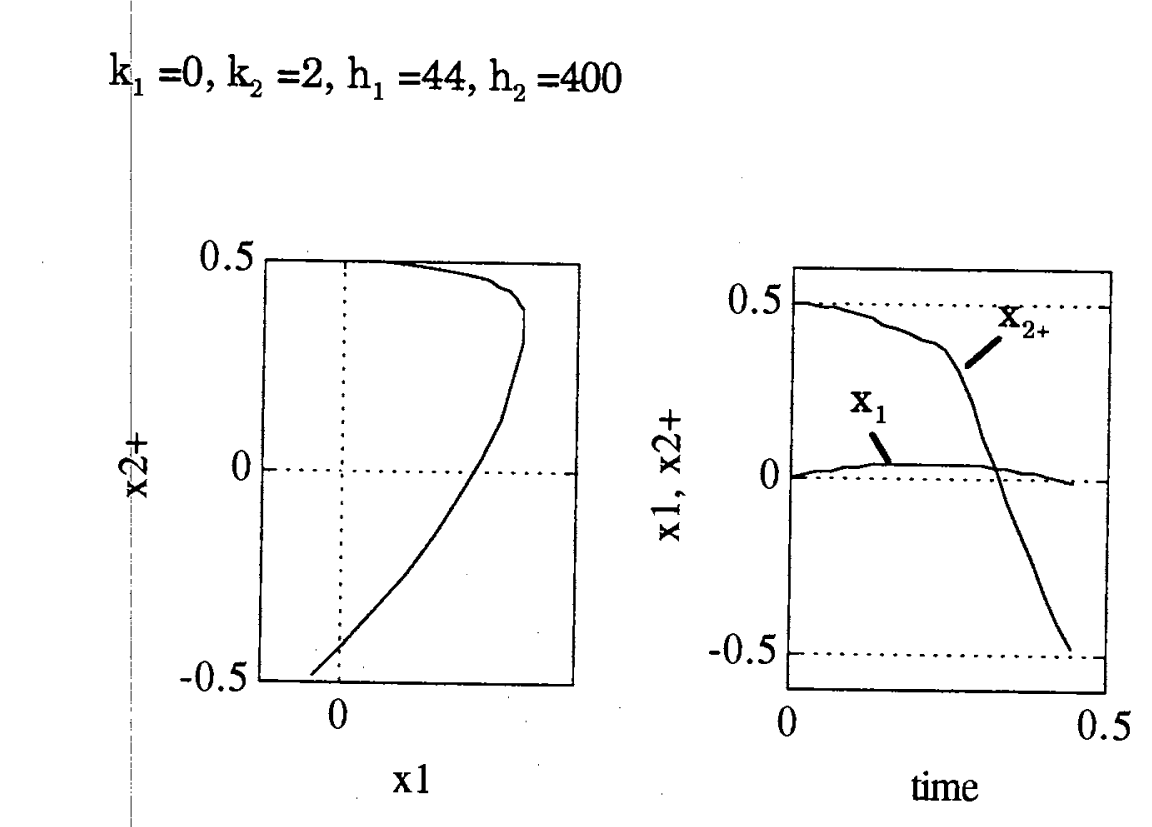

Figure 4.9 Simulation results of SOON

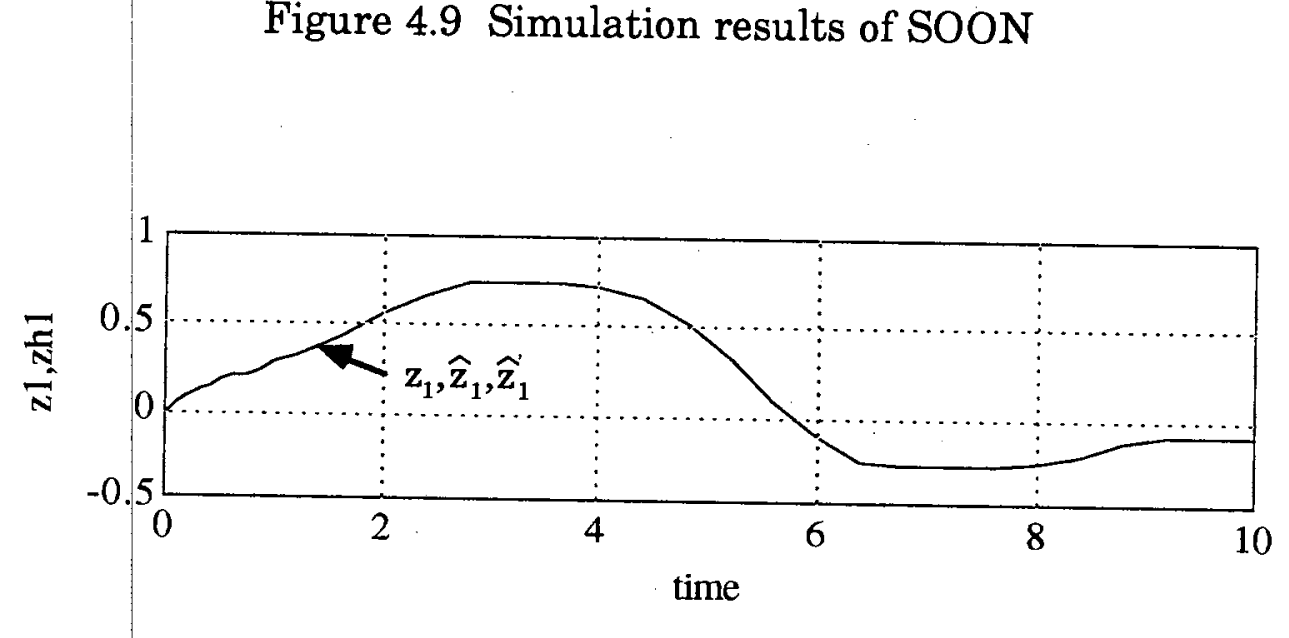

Figure 4.10 Simulation results: time domain plot of 1st states 


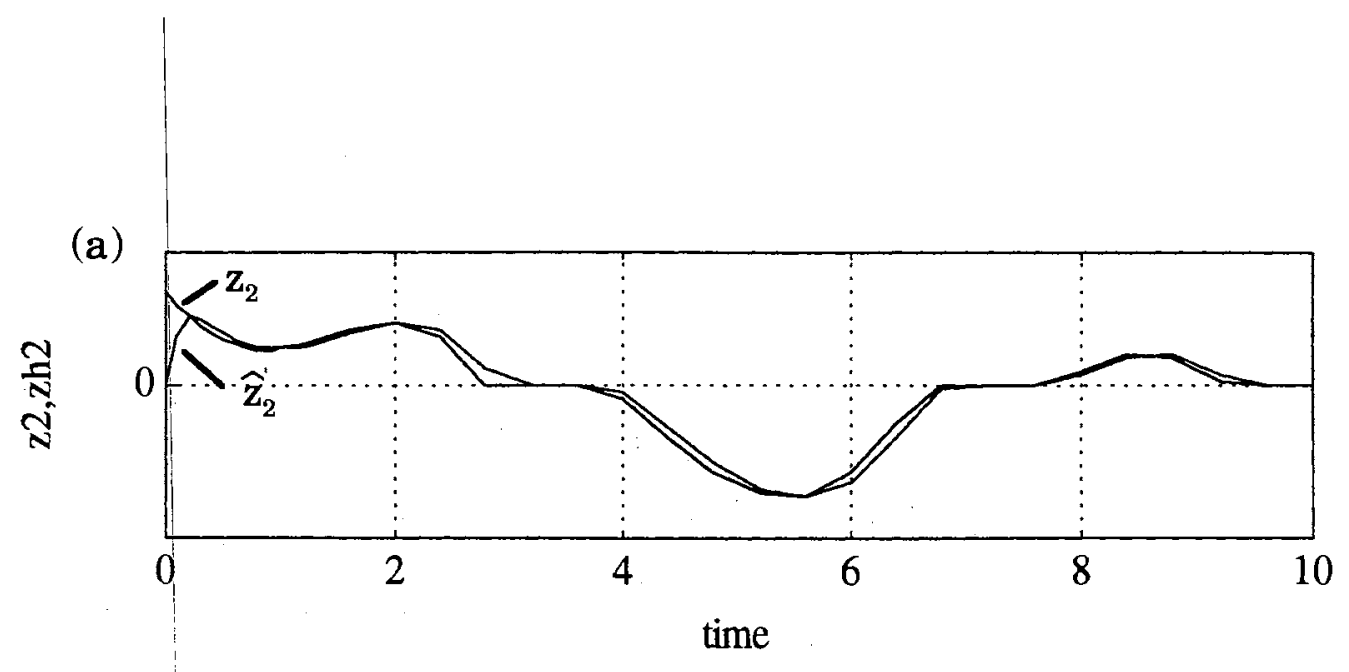

91

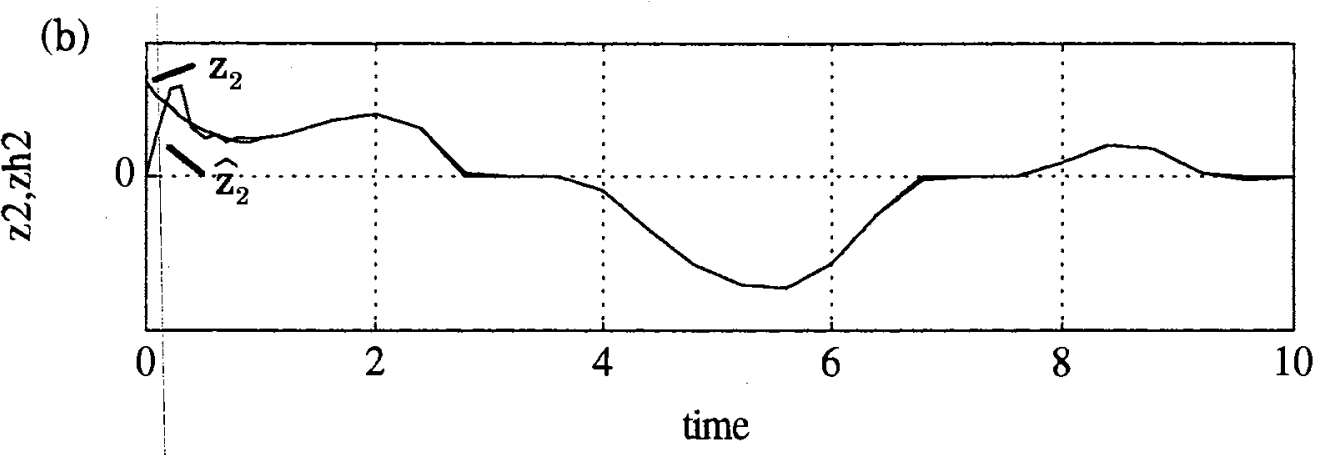

Figure 4.11 Simulation results: time domain plot of 2 nd states
a) The sliding observer by absolute stability theorem
b) The sliding observer by SOON

Even though the both of simulation results satisfy the design goal, we can see the difference between them. Both of the first estimated state are so close to that of actual system, we cannot see the difference in Figure 4.10. The saturation sliding observer is equivalently high gain system within the boundary layer. On the contrary, if the signum sliding observer is arrived at sliding patch, its dynamics is equivalently reduced order system and its steady state error bounds are limited by the shifted-coefficient as shown in the equation (2.43). 


\subsection{Super-tanker Lateral Dynamics}

For the 3-order example, the sliding observer is used to estimate the angular velocity (yaw rate) and the angular acceleration of a super-tanker from the measurement of the "heading", i.e., the yaw angle. The equation of motion is originally derived by Frimm [1983]. The plant is described as follows:

$$
\left\{\begin{array}{l}
\dot{z}_{1}=z_{2} \\
\dot{z}_{2}=z_{3} \\
\dot{z}_{3}=-\frac{\kappa}{T_{1} T_{2}} H\left(z_{2}\right)-\left(\frac{1}{T_{1}}+\frac{1}{T_{2}}\right) z_{3}+\frac{\kappa}{T_{1} T_{2}}\left(\delta+T_{3} \dot{\delta}\right)
\end{array}\right.
$$

where

- $\mathrm{z}_{1}=\psi$ is the yaw angle (degree)

- $z_{2}=\dot{\psi}$ is the yaw rate (degree/sec)

- $z_{3}$ is the derivative of yaw rate

- $\delta$ is the rudder angle (degree)

The function $\mathrm{H}\left(\mathrm{z}_{2}\right)=\mathrm{H}(\dot{\psi})$ and the constants were identified from the actual ship as:

$$
\begin{aligned}
& \mathrm{H}(\dot{\psi})=1.8419-21.294 \dot{\psi}-8.0534 \dot{\psi}^{2}+96.5283 \dot{\psi}^{3}-24.9247 \dot{\psi}^{5} \\
& \text { - } \mathrm{T}_{1}=-60.26 \\
& \text { - } \mathrm{T}_{2}=7.77 \\
& \text { - } \mathrm{T}_{3}=17.5 \\
& \text { - } \kappa=-0.04696
\end{aligned}
$$

The goal of this example is to design a sliding observer which estimates the second and third states with the only available measurement of yaw angle. A linear model by Arie will be used and the rudder inputs will be 
lumped with the nonlinearities and regarded as an uncertainty.

The model used for the observer design is

where $|\mathrm{w}| \leq \gamma$

$$
\left\{\begin{array}{l}
\dot{z}_{1}=z_{2} \\
\dot{z}_{2}=z_{3} \\
\dot{z}_{3}=-\frac{1}{\mathrm{~T}_{1} \mathrm{~T}_{2}} \mathrm{z}_{2}-\left(\frac{1}{\mathrm{~T}_{1}}+\frac{1}{\mathrm{~T}_{2}}\right) \mathrm{z}_{3}+\mathrm{w}
\end{array}\right.
$$

The uncertainty bound $\gamma$ will be determined based on the knowledge of the actual system. The suggested sliding observer is

$$
\left\{\begin{array}{l}
\dot{\hat{z}}_{1}=\widehat{z}_{2}+h_{1} x_{1}+k_{1} \operatorname{sgn}\left(x_{1}\right) \\
\dot{\hat{z}_{2}}=\widehat{z}_{3}+h_{2} x_{1}+k_{2} \operatorname{sgn}\left(x_{1}\right) \\
\dot{\mathrm{z}}_{3}=-\frac{1}{\mathrm{~T}_{1} \mathrm{~T}_{2}} \widehat{\mathrm{z}}_{2}-\left(\frac{1}{\mathrm{~T}_{1}}+\frac{1}{\mathrm{~T}_{2}}\right) \hat{\mathrm{z}}_{3}+\mathrm{h}_{3} \mathrm{x}_{1}+\mathrm{k}_{3} \operatorname{sgn}\left(\mathrm{x}_{1}\right)
\end{array}\right.
$$

where $x_{1}=z_{1}-\hat{z}_{1}$

To follow the design procedure, the uncertainty bound needs to be evaluated first. Since modeling errors of this example are mathematically unbounded, it is necessary to use the physical knowledge to determine the bounds for modeling errors. The experimental data are as follows:

- $\left|z_{1}\right|<1$ (degree)

- $\left|z_{2}\right|<0.2$ (degree/sec)

- $|\delta| \leq 10$ (degree)

Using the physical bounds given above, the uncertainty bound is obtained via the Schwarz inequalities.

$$
|\mathrm{w}|=\left|\frac{1}{\mathrm{~T}_{1} \mathrm{~T}_{2}}\left(\mathrm{z}_{2}-\kappa \mathrm{H}\left(\mathrm{z}_{2}\right)\right)+\frac{\kappa}{\mathrm{T}_{1} \mathrm{~T}_{2}}\left(\delta+\mathrm{T}_{3} \dot{\delta}\right)\right|<.023
$$

With $\kappa=0.1$, take the bound as $\gamma=0.023$. The next step is to determine 
the bounds on the initial error states. Let assume the bounds as follows:

- $\mathbf{x}_{1} \approx 0$

- $\left|\mathrm{x}_{2}\right|_{\max } \leq 1.5$

- $\left|\mathrm{x}_{3}\right|_{\max } \leq 0.5$

The next step is choosing the linear correction coefficient. Simply pole place all at -2 and make it slightly underdamped to be captured in the sliding patch fast. The chosen coefficient is

$$
\mathrm{H}=\left[\begin{array}{lll}
6 & 12.2 & 8.4
\end{array}\right]^{\mathrm{T}}
$$

With this design constant, simulate the reaching dynamics to get the worst final passing point. Considering the bound of initial state, 2 different initial conditions are simulate and the results are shown in following Figures.
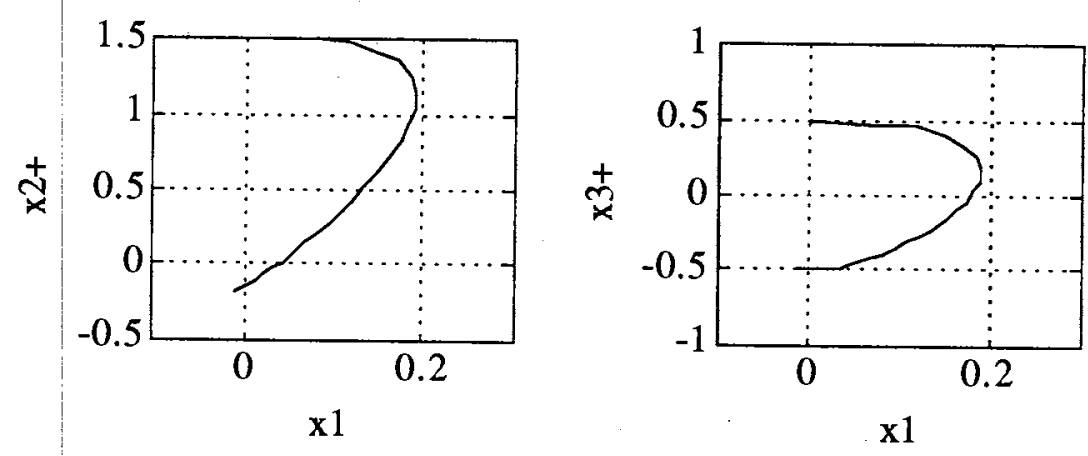

Figure 4.12 Worst reaching dynamics with $x^{\mathrm{i}}=\left[\begin{array}{lll}0 & 1.5 & 0.5\end{array}\right]$
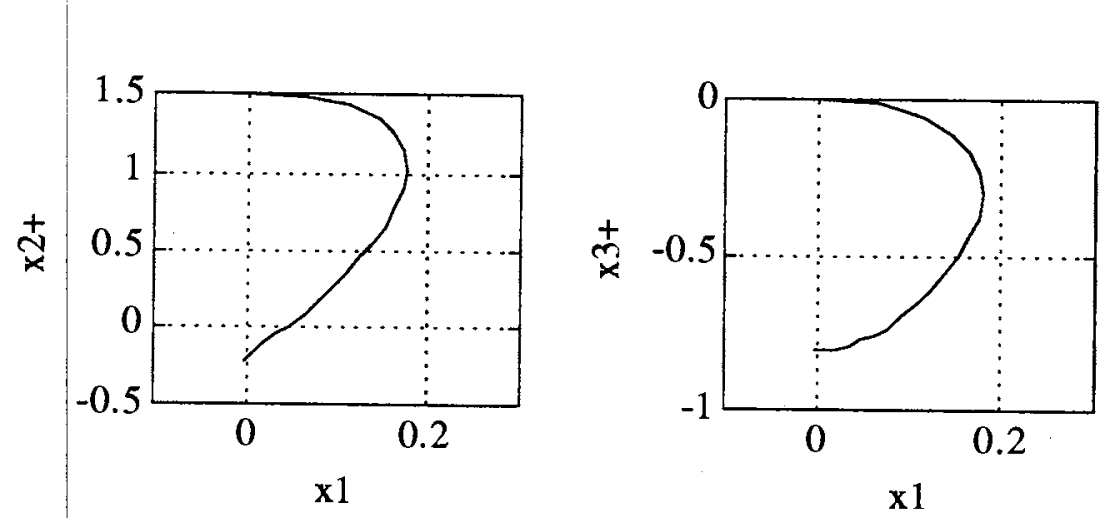

Figure 4.13 Worst reaching dynamics with $x^{i}=\left[\begin{array}{lll}0 & 1.5 & 0\end{array}\right]$ 
The simulation shows that the later one is worse than the first one whose initial Lyapunov-like function is greater than that of later one. The final passing point are $\sigma_{+}^{\mathrm{f}}=\left[\begin{array}{llll}0 & -0.18 & -0.49\end{array}\right]^{\mathrm{T}}$ and $\sigma_{+}^{\mathrm{f}}=\left[\begin{array}{lll}0 & -0.24 & -0.8\end{array}\right]^{\mathrm{T}}$. However, the both results satisfy the requirement of stability Theorem 3.2. Assigning the shifted-coefficient $\mathrm{K}_{\mathrm{s}}$ as half of the worst final passing state will guarantee one cycle convergence. Considering the equivalent sliding dynamics eigenvalues, the switching coefficient is chosen as:
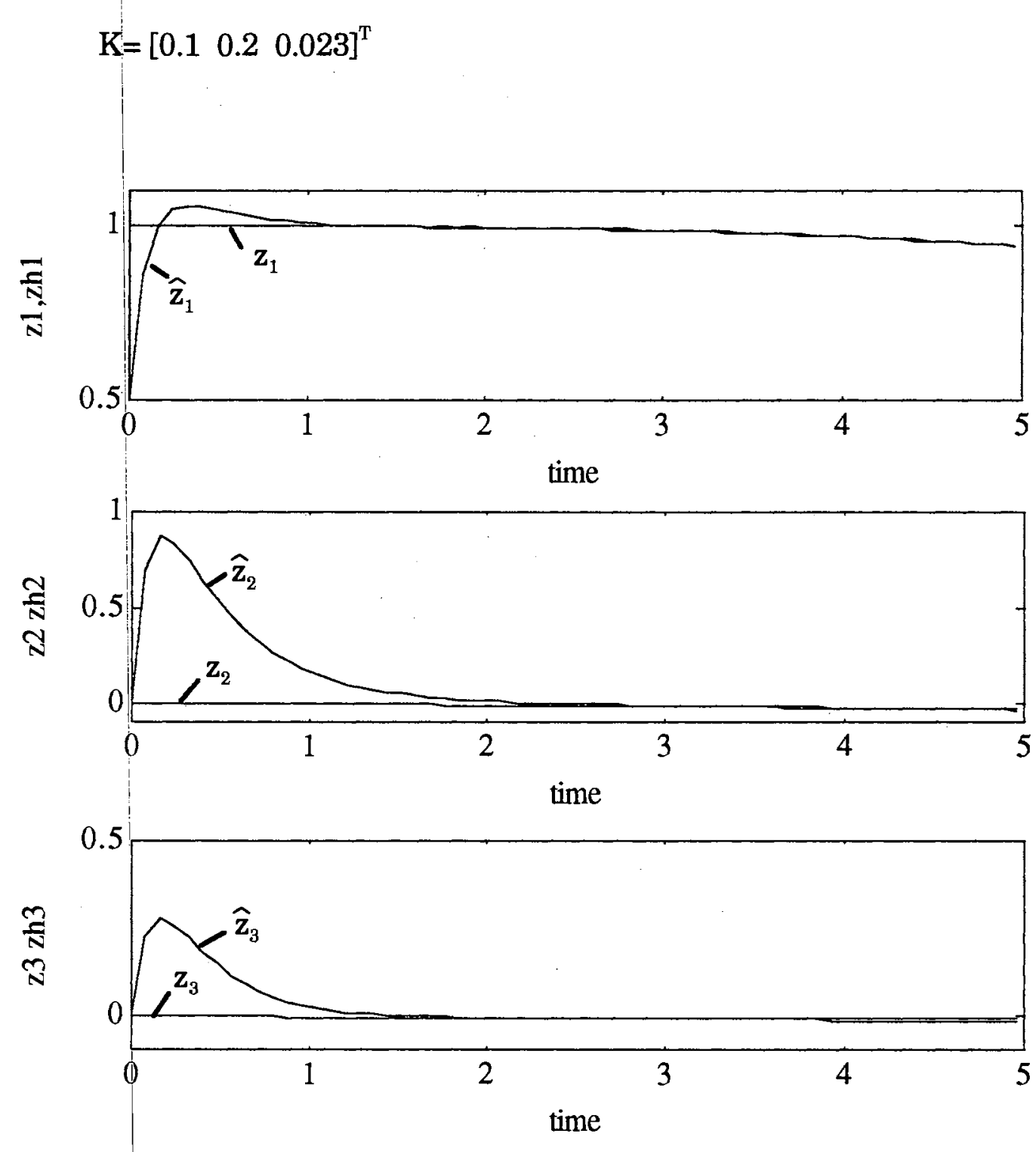

Figure 4.14 The actual and estimated states of super-tanker 
Even the bound of the rudder angle is $10^{\circ}$, the simulation is done for $\delta$ $=30^{\circ}$ to demonstrate "robustness" of the designed sliding observer as shown in Figure 4.14 in time domain. It shows that the solution point is captured by the sliding patch within one cycle as expected. 


\section{CHAPTER V}

\section{ROBOT CONTROL BASED ON SLIDING OBSERVERS}

\subsection{Sliding Observer for Multiple Measurements}

In the state estimation problem, the need to handle model uncertainty due to nonlinearities or disturbance leads to the development of a sliding observer. A sliding observer is one of the simple robust estimations. Despite the presence of uncertainty and disturbances whose bound is known it is easy to show that the performance of the sliding observer is theoretically perfect.

The sliding observer with multiple measurement in phase variable canonical form can be designed as far as the disturbance input bound of the error dynamics is known. The system with double measurements is assumed as:

$$
\begin{aligned}
& \dot{\mathrm{z}}_{1}=\mathrm{z}_{2} \\
& \dot{\mathrm{z}}_{2}=\mathrm{g}_{1}\left(\mathrm{z}, \mathrm{u}_{1}, \mathrm{t}, \mathrm{w}_{1}, \theta\right) \\
& \dot{\mathrm{z}}_{3}=\mathrm{z}_{4} \\
& \dot{\mathrm{z}}_{4}=\mathrm{g}_{2}\left(\mathrm{z}, \mathrm{u}_{2}, \mathrm{t}, w_{2}, \theta\right)
\end{aligned}
$$

The suggested sliding observer is:

$$
\left(\begin{array}{l}
\dot{\mathrm{z}}_{1}=\widehat{\mathrm{z}}_{2}+\mathrm{h}_{1}\left(\mathrm{y}_{1}-\mathrm{C}_{1} \hat{\mathrm{z}}\right)+\mathrm{k}_{1} \operatorname{sgn}\left(\mathrm{x}_{1}\right) \\
\dot{\mathrm{z}}_{2}=\widehat{\mathrm{g}}_{1}+\mathrm{h}_{2}\left(\mathrm{y}_{1}-\mathrm{C}_{1} \widehat{\mathrm{z}}\right)+\mathrm{k}_{2} \operatorname{sgn}\left(\mathrm{x}_{1}\right) \\
\dot{\hat{\mathrm{z}}}_{3}=\widehat{\mathrm{z}}_{4}+\mathrm{h}_{3}\left(\mathrm{y}_{2}-\mathrm{C}_{2} \hat{\mathrm{z}}\right)+\mathrm{k}_{3} \operatorname{sgn}\left(\mathrm{x}_{3}\right) \\
\dot{\hat{\mathrm{z}}}_{4}=\widehat{\mathrm{g}}_{2}+\mathrm{h}_{4}\left(\mathrm{y}_{2}-\mathrm{C}_{2} \widehat{\mathrm{z}}\right)+\mathrm{k}_{4} \operatorname{sgn}\left(\mathrm{x}_{3}\right)
\end{array}\right.
$$


where $\mathrm{x}_{1}=\mathrm{y}_{1}-\mathrm{C}_{1} \hat{\mathrm{z}}$ and $\mathrm{x}_{3}=\mathrm{y}_{2}-\mathrm{C}_{2} \hat{\mathrm{z}}$

The error dynamics:

$$
\left\{\begin{array}{l}
\dot{\mathrm{x}}_{1}=\mathrm{x}_{2}-\mathrm{h}_{1} \mathrm{x}_{1}-\mathrm{k}_{1} \operatorname{sgn}\left(\mathrm{x}_{1}\right) \\
\dot{\mathrm{x}}_{2}=\mathrm{w}_{1}-\mathrm{h}_{2} \mathrm{x}_{1}-\mathrm{k}_{2} \operatorname{sgn}\left(\mathrm{x}_{1}\right) \\
\dot{\mathrm{x}}_{3}=\mathrm{x}_{4}-\mathrm{h}_{3} \mathrm{x}_{3}-\mathrm{k}_{3} \operatorname{sgn}\left(\mathrm{x}_{3}\right) \\
\dot{\mathrm{x}}_{4}=\mathrm{w}_{2}-\mathrm{h}_{4} \mathrm{x}_{3}-\mathrm{k}_{4} \operatorname{sgn}\left(\mathrm{x}_{3}\right)
\end{array}\right.
$$

where $\mathrm{w}_{1}=\mathrm{g}_{1}\left(\mathrm{z}, \mathrm{u}_{1}, \mathrm{t}, w_{1}, \theta\right)-\widehat{\mathrm{g}}_{1}\left(\widehat{\mathrm{z}}, \mathrm{u}_{1}, \mathrm{t}, \hat{\theta}\right)$ and $\mathrm{w}_{2}=\mathrm{g}_{2}\left(\mathrm{z}, \mathrm{u}_{2}, \mathrm{t}, w_{2}, \theta\right)-\hat{\mathrm{g}}_{2}\left(\widehat{\mathrm{z}}, \mathrm{u}_{2}, \mathrm{t}, \hat{\theta}\right)$

For the sliding observer error dynamics, $\mathrm{w}_{1}$ and $\mathrm{w}_{2}$ are the disturbance inputs which include neglected nonlinearities, parametric uncertainties, modeling errors and noises. The bound of the disturbance input $w_{1}$ and $w_{2}$ are assumed to be known. If the error dynamics of multiple measurements system can be decoupled, then the sliding observer can be designed exactly the same method as the single measurement case. Compared to the single measurements sliding observer, the multiple measurements sliding observer has multiple hyperplanes. This is one of the main difference between the sliding observer and sliding control problem.

In this chapter, the sliding observer is used to estimate the states of a two-link manipulator for multiple measurement example. Since every observer is considered to be a part of a closed-loop feedback system, as a practical example, a sliding observer for a two-link robot system was designed and simulated to compose a closed-loop feedback system with several control method. If the model of robot is known exactly, the nonlinear term of the equations of motion can be included in the sliding observer for an accurate estimation. For this case, the Feedback Linearization method can be applied to control the nonlinear system. And also the SOFL (Feedback Linearization control based on Sliding Observer) is composed and simulated. If the 
dynamics is not known exactly, the Feedback Linearization control does not work properly. Also, for the sliding observer, the simplified model may be preferred. For the system control, the adaptive control and the sliding control were designed.

\subsection{Robots Dynamics}

\subsubsection{Dynamics of Rigid Robots}

The mathematical model for a n-link robot is derived via the EulerLaglangian equations (see Spong and Vidyasagar for details [Spong, 1989]):

$$
\sum_{j} d_{k j}(q) \ddot{q}_{j}+\sum_{i, j} C_{i j k}(q) \dot{q}_{i} \dot{q}_{j}+\phi_{k}(q)=\tau_{k}
$$

where $d_{k j}$; The coefficient of inertia matrix $D(q)$

$\phi_{\mathrm{k}} ;$ The gravitational forces and torques

$\mathrm{c}_{i \mathrm{jk}}$; The coriolis and centrifugal terms

$$
\mathrm{C}_{\mathrm{ijk} k}=\frac{1}{2}\left\{\frac{\partial \mathrm{d}_{\mathrm{kj}}}{\partial \mathrm{q}_{\mathrm{i}}}+\frac{\partial \mathrm{d}_{\mathrm{ki}}}{\partial \mathrm{q}_{\mathrm{j}}}-\frac{\partial \mathrm{d}_{\mathrm{ij}}}{\partial \mathrm{q}_{\mathrm{k}}}\right\}
$$

Equation (5.4) can be rewritten in the matrix form:

$$
\mathrm{D}(\mathrm{q}) \ddot{\mathrm{q}}+\mathrm{C}(\mathrm{q}, \dot{\mathrm{q}}) \dot{\mathrm{q}}+\mathrm{g}(\mathrm{q})=\tau
$$

where the $k, j_{-}$th element of the matrix $C(q, \dot{q})$ is defined as:

$$
\begin{aligned}
& \mathrm{C}_{\mathrm{kj}}=\sum_{\mathrm{i}=1}^{\mathrm{n}} \mathrm{C}_{\mathrm{ijk}}(\mathrm{q}) \dot{\mathrm{q}}_{\mathrm{i}} \\
& =\sum_{\mathrm{i}=1}^{\mathrm{n}} \frac{1}{2}\left\{\frac{\partial \mathrm{d}_{\mathrm{kj}}}{\partial \mathrm{q}_{\mathrm{i}}}+\frac{\partial \mathrm{d}_{\mathrm{ki}}}{\partial \mathrm{q}_{\mathrm{j}}}-\frac{\partial \mathrm{d}_{\mathrm{j}}}{\partial \mathrm{q}_{\mathrm{k}}}\right\} \dot{\mathrm{q}}_{\mathrm{i}}
\end{aligned}
$$

and the $\mathrm{k}_{-}$th component of $\mathrm{g}$ is $\phi_{\mathrm{k}}$

For a general n-link manipulator, the equations of motion (5.4) are 
nonlinear and coupled, but for the simplest robots, they have several properties [Spong, 1990] that are useful for designing control system.

\section{Property}

1) The inertia matrix $D(q)$ is symmetric, positive definite, and both $D(q)$ and $\mathrm{D}(\mathrm{q})^{-1}$ are uniformly bounded as a function of $\mathrm{q} \in \mathrm{R}^{\mathrm{n}}$

2) There is an independent control input for each degree of freedom.

3) Link masses, moments of inertias etc., appear as coefficients of known functions of the generalized coordinates.

4) The Coriolis and centrifugal forces are quadratic in the terms of $\dot{q}_{i}$

$$
\|\mathrm{C}(\mathrm{q}, \dot{\mathrm{q}}) \dot{\mathrm{q}}\| \leq \sigma_{1}\|\dot{\mathrm{q}}\|^{2}
$$

5) The vector $\mathrm{C}(\mathrm{q}, \dot{\mathrm{q}}) \dot{\mathrm{q}}$ has elements $\dot{\mathrm{q}}^{\mathrm{T}} \mathrm{N}_{\mathrm{i}}(\mathrm{q}) \dot{\mathrm{q}}$ where the $\mathrm{N}_{\mathrm{i}}$ 's matrices are symmetric and composed of bounded periodic elements.

Each term of the matrices of dynamic equation (5.4) can be defined as a separate parameter, so that the dynamic equation (5.5) is rewritten in a linear regression form:

$$
\mathrm{D}(\mathrm{q}) \ddot{\mathrm{q}}+\mathrm{C}(\mathrm{q}, \dot{\mathrm{q}}) \dot{\mathrm{q}}+\mathrm{g}(\mathrm{q})=\mathrm{Y}(\mathrm{q}, \dot{\mathrm{q}}, \ddot{\mathrm{q}}) \theta=\tau
$$

where $\mathrm{Y}(\mathrm{q}, \dot{\mathrm{q}}, \ddot{\mathrm{q}})$ is an $\mathrm{n} \times \mathrm{r}$ matrix of known functions, known as the regressor, and $\mathrm{q}$ is an $\mathrm{r}$-order vector of parameters.

\subsubsection{Two Link Manipulator}

Fig. 5.1 is a planar type model of two-link robot with a motor at each joint for control input. The dynamic equation (5.3) for a two-link robot in matrix form is:

$$
\begin{aligned}
& \mathrm{d}_{11} \ddot{\mathrm{q}}_{1}+\mathrm{d}_{12} \ddot{\mathrm{q}}_{2}+\mathrm{C}_{121} \dot{\mathrm{q}}_{1} \dot{\mathrm{q}}_{2}+\mathrm{C}_{211} \dot{\mathrm{q}}_{2} \dot{\mathrm{q}}_{1}+\mathrm{C}_{211} \dot{\mathrm{q}}_{2}^{2}+\phi_{1}=\tau_{1} \\
& \mathrm{~d}_{21} \ddot{\mathrm{q}}_{1}+\mathrm{d}_{22} \ddot{\mathrm{q}}_{2}+\mathrm{C}_{112} \dot{\mathrm{q}}_{1}^{2}+\phi_{2}=\tau_{2}
\end{aligned}
$$


where $\mathrm{i}=1,2$

$\tau_{\mathrm{i}} ;$ The control inputs

$\mathrm{q}_{\mathrm{i}}$; A generalized coordinate that is the joint angle

$\mathrm{d}_{\mathrm{11}}=\mathrm{m}_{1} 1_{\mathrm{c} 1}{ }^{2}+\mathrm{m}_{2}\left(\mathrm{l}_{1}{ }^{2}+\mathrm{l}_{\mathrm{c} 2}{ }^{2}+2 \mathrm{l}_{1} \mathrm{l}_{\mathrm{c} 2} \cos \left(\mathrm{q}_{2}\right)\right)+\mathrm{I}_{1}+\mathrm{I}_{2}$

$\mathrm{d}_{12}=\mathrm{d}_{21}=\mathrm{m}_{2}\left(\mathrm{l}_{\mathrm{c} 2}{ }^{2}+\mathrm{l}_{1} \mathrm{l}_{\mathrm{c} 2} \cos \left(\mathrm{q}_{2}\right)\right)+\mathrm{I}_{2}$

$\mathrm{d}_{\mathfrak{2}}=\mathrm{m}_{2} \mathrm{l}_{\mathrm{c} 2}^{2}+\mathrm{I}_{2}$

$c_{121}=c_{211}=c_{221}=-c_{112}=m_{2} l_{1} l_{c 2} \sin \left(q_{2}\right):=h$

$\phi_{1}=\left(m_{1} l_{c 1}+m_{2} l_{1}\right) g \cos \left(q_{1}\right)+m_{2} l_{c 2} g \cos \left(q_{1}+q_{2}\right)$

$\phi_{2}=m_{2} l_{c 2} g \cos \left(q_{1}+q_{2}\right)$ where $I_{i}$; The moment of inertia of link $i$

is at the center of mass of link $i$

$1_{\mathrm{ci}}$; The distance from the previous joint to the center of mass link $\mathrm{i}$

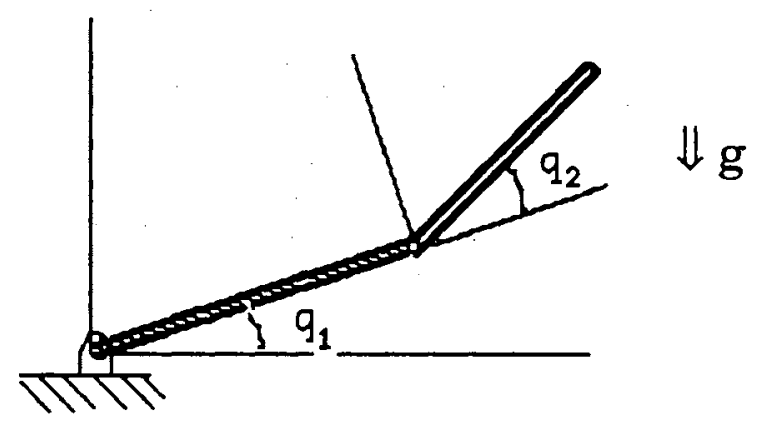

Figure 5.1. Two link Manipulator

Let us define parameters $\theta_{1}, \ldots, \theta_{9}$ to get a linear regression equation:

$$
\begin{array}{lll}
\theta_{1}=\mathrm{m}_{1} \mathrm{l}_{\mathrm{c} 1}{ }^{2}, & \theta_{4}=\mathrm{m}_{2} \mathrm{l}_{1} \mathrm{l}_{\mathrm{c} 2}, & \theta_{7}=\mathrm{m}_{1} \mathrm{l}_{\mathrm{c} 1} \mathrm{~g} \\
\theta_{2}=\mathrm{m}_{2} \mathrm{l}_{1}^{2}, & \theta_{5}=\mathrm{I}_{1}, & \theta_{8}=\mathrm{m}_{2} \mathrm{l}_{1} \mathrm{~g} \\
\theta_{3}=\mathrm{m}_{2} \mathrm{l}_{\mathrm{c} 2}{ }^{2}, & \theta_{6}=\mathrm{I}_{2}, & \theta_{9}=\mathrm{m}_{2} \mathrm{l}_{\mathrm{c} 2} \mathrm{~g}
\end{array}
$$

where $\theta=\left[\theta_{1}, \ldots, \theta_{9}\right]^{\mathrm{T}}$

Using the parameter (5.7), rewrite the equation (5.6) in a linear regression 
form:

$$
\begin{aligned}
& \mathrm{Y}(\mathrm{q}, \dot{\mathrm{q}}, \ddot{\mathrm{q}}) \theta=\tau \\
& \mathrm{Y}_{1}(\mathrm{q}, \dot{\mathrm{q}}, \ddot{\mathrm{q}}) \theta_{\mathrm{n}}+\mathrm{Y}_{2}(\mathrm{q}, \dot{\mathrm{q}}, \ddot{\mathrm{q}}) \theta=\tau
\end{aligned}
$$

where $\theta_{\mathrm{n}}$ : known parameter, $\theta$ : to be estimated

Assuming that $\mathrm{m}_{1}$ is known exactly and does not change, load and disturbances are included in $\mathrm{m}_{2}$, so that $\mathrm{m}_{2}$ is unknown. If the equation (5.9) is applied to a SCARA (for Selective Compliant Articulated Robot for Assembly) type robot, the gravitational terms $\theta_{7}, \theta_{8}, \theta_{9}$. will not appear.

The equations of motion (5.6) is rewritten in a state space form. First, the equation (5.6) is multiplied by the inverse of inertia matrix and we have:

$$
\begin{aligned}
& \dot{\mathrm{z}}_{1}=\mathrm{z}_{2} \\
& \dot{\mathrm{z}}_{2}=\overline{\mathrm{c}}_{11} \mathrm{z}_{2}+\overline{\mathrm{c}}_{12} \mathrm{z}_{4}+\bar{\phi}_{1}+\bar{\tau}_{1} \\
& \dot{\mathrm{z}}_{3}=\mathrm{z}_{4} \\
& \dot{\mathrm{z}}_{4}=\overline{\mathrm{c}}_{21} \mathrm{z}_{2}+\overline{\mathrm{c}}_{22} \mathrm{z}_{4}+\bar{\phi}_{2}+\bar{\tau}_{2}
\end{aligned}
$$

where $z_{1}=q_{1}, z_{2}=\dot{q}_{1}, z_{3}=q_{2}$, and $z_{4}=\dot{q}_{2}$

$$
\begin{aligned}
& \Delta=d_{11} d_{22}-d_{12} d_{21} ; \text { determinant of inertia matrix } D(q) \\
& \bar{c}_{11}=-\left(d_{22} c_{11}-d_{12} c_{21}\right) / \Delta \\
& \bar{c}_{12}=-\left(d_{22} c_{12}-d_{12} c_{22}\right) / \Delta \\
& \bar{c}_{21}=-\left(d_{11} c_{21}-d_{12} c_{11}\right) / \Delta \\
& \bar{c}_{22}=-\left(d_{11} c_{22}-d_{12} c_{12}\right) / \Delta \\
& \bar{\phi}_{1}=-\left(d_{22} \phi_{1}-d_{12} \phi_{2}\right) / \Delta \\
& \overline{\phi_{2}}=-\left(-d_{12} \phi_{1}+d_{11} \phi_{2}\right) / \Delta \\
& \bar{\tau}_{1}=\left(d_{22} \tau_{1}-d_{12} \tau_{2}\right) / \Delta \\
& \bar{\tau}_{2}=\left(-d_{12} \tau_{1}+d_{11} \tau_{2}\right) / \Delta
\end{aligned}
$$


where $q_{1}$ and $q_{2}$ are the two joint angles, $\tau_{1}$ and $\tau_{2}$ are the joint inputs, and

$$
\begin{aligned}
& \mathrm{d}_{11}=\mathrm{m}_{1} \mathrm{l}_{\mathrm{c} 1}{ }^{2}+\mathrm{m}_{2}\left(\mathrm{l}_{1}^{2}+\mathrm{l}_{\mathrm{c} 2}{ }^{2}+21_{1} \mathrm{l}_{\mathrm{c} 2} \cos \left(\mathrm{z}_{3}\right)\right)+\mathrm{I}_{1}+\mathrm{I}_{2} \\
& \mathrm{~d}_{12}=\mathrm{d}_{2 \mathrm{1}}=\mathrm{m}_{2}\left(\mathrm{l}_{\mathrm{c} 2}{ }^{2}+\mathrm{l}_{1} \mathrm{l}_{\mathrm{c} 2} \cos \left(\mathrm{z}_{3}\right)\right)+\mathrm{I}_{2} \\
& \mathrm{~d}_{\mathfrak{2}}=\mathrm{m}_{2} \mathrm{l}_{\mathrm{c} 2}{ }^{2}+\mathrm{I}_{2} \\
& \mathrm{~h}:=\mathrm{m}_{2} \mathrm{l}_{1} \mathrm{l}_{\mathrm{c} 2} \sin \left(\mathrm{z}_{3}\right) \\
& \mathrm{c}_{11}=-\mathrm{h} \mathrm{z}_{4} \\
& \mathrm{c}_{12}=-\mathrm{hz}_{2}-\mathrm{hz}_{4} \\
& \mathrm{c}_{21}=\mathrm{h} \mathrm{z} \\
& \mathrm{c}_{22}=0 \\
& \phi_{1}=\left(m_{1} l_{c 1}+m_{2} l_{1}\right) g \cos \left(z_{1}\right)+m_{2} l_{c 2} g \cos \left(z_{1}+z_{2}\right) \\
& \phi_{2}=m_{2} 1_{c 2} g \cos \left(z_{1}+z_{2}\right)
\end{aligned}
$$

The specification of a two-link manipulator for the computational simulation is $\mathrm{m}_{1}=20, \mathrm{~m}_{2}=10, \mathrm{l}_{1}=1, \mathrm{c}_{\mathrm{c}^{1}}=0.5, \mathrm{l}_{2}=0.8, \mathrm{c}^{2}=0.5, \mathrm{I}_{1}=5, \mathrm{I}_{2}=$ $2.5, \mathrm{~g}=9.8$, with the SI unit.

\subsection{The Case with No Parameter Uncertainty}

\subsubsection{The Design of the Sliding Observer}

Even though we know the system exactly, the estimated parameters may be different from the actual value, since the parameters are function of estimated states. In this section, the nonlinear term is considered to design a sliding observer. The observer is obtained by replacing the parameters in (5.10) with estimated parameters.

$$
\begin{aligned}
& \dot{\hat{\mathrm{z}}}_{1}=\widehat{\mathrm{z}}_{2}+\mathrm{h}_{1} \widetilde{\mathrm{y}}_{1}+\mathrm{k}_{1} 1_{\mathrm{s}}\left(\widetilde{\mathrm{y}}_{1}\right) \\
& \dot{\hat{\mathrm{z}}}=\mathrm{h}_{2} \widetilde{\mathrm{y}}_{1}+\mathrm{k}_{2} 1_{\mathrm{s}}\left(\widetilde{\mathrm{y}}_{1}\right)+\hat{\overline{\mathrm{c}}}_{11} \widehat{\mathrm{z}}_{2}+\hat{\overline{\mathrm{c}}}_{12} \widehat{\mathrm{z}}_{4}+\hat{\bar{\phi}}_{1}+\hat{\bar{\tau}_{1}} \\
& \dot{\hat{\mathrm{z}}_{3}}=\hat{\mathrm{z}}_{4}+\mathrm{h}_{3} \widetilde{\mathrm{y}}_{2}+\mathrm{k}_{3} 1_{\mathrm{s}}\left(\widetilde{\mathrm{y}}_{2}\right)
\end{aligned}
$$




$$
\hat{\mathrm{z}}_{4}=h_{4} \widetilde{\mathrm{y}}_{2}+\mathrm{k}_{4} 1_{\mathrm{s}}\left(\widetilde{\mathrm{y}}_{2}\right)+\widehat{\overline{\mathrm{c}}}_{21} \widehat{\mathrm{z}}_{2}+\widehat{\overline{\mathrm{c}}}_{22} \widehat{\mathrm{z}}_{4}+\hat{\overline{\phi_{2}}}+\hat{\overline{\bar{\tau}}}
$$

where $\widetilde{y}_{1}=x_{1}=z_{1}-\hat{z}_{1}$ and $\widetilde{y}_{2}=x_{3}=z_{3}-\hat{z}_{3}$

The error dynamics equations are

$$
\begin{aligned}
& \dot{\mathrm{x}}_{1}=\mathrm{x}_{2}-\mathrm{h}_{1} \mathrm{x}_{1}-\mathrm{k}_{1} 1_{\mathrm{s}}\left(\mathrm{x}_{1}\right) \\
& \dot{\mathrm{x}}_{2}=-\mathrm{h}_{2} \mathrm{x}_{1}-\mathrm{k}_{2} 1_{\mathrm{s}}\left(\mathrm{x}_{1}\right)+\mathrm{w}_{1} \\
& \dot{\mathrm{x}}_{3}=\mathrm{x}_{4}-\mathrm{h}_{3} \mathrm{x}_{3}-\mathrm{k}_{3} 1_{\mathrm{s}}\left(\mathrm{x}_{3}\right) \\
& \dot{\mathrm{x}}_{4}=-\mathrm{h}_{4} \mathrm{x}_{3}-\mathrm{k}_{4} 1_{\mathrm{s}}\left(\mathrm{x}_{3}\right)+\mathrm{w}_{2}
\end{aligned}
$$

where $x_{1}=z_{1}-\hat{z}_{1}, x_{3}=z_{3}-\hat{z}_{3}$ and

$$
\begin{aligned}
& \mathrm{w}_{1}=\overline{\mathrm{c}}_{11} \mathrm{x}_{2}+\left(\overline{\mathrm{c}}_{11}-\hat{\overline{\mathrm{c}}}_{11}\right) \hat{\mathrm{z}}_{2}+\overline{\mathrm{c}}_{12} \mathrm{x}_{4}+\left(\overline{\mathrm{c}}_{12}-\hat{\overline{\mathrm{c}}}_{12}\right) \widehat{\mathrm{z}}_{4}+\bar{\phi}_{1}-\hat{\bar{\phi}}+\overline{\boldsymbol{\tau}}_{1}-\hat{\bar{\tau}} \\
& \mathrm{w}_{2}=\overline{\mathrm{c}}_{21} \mathrm{x}_{2}+\left(\overline{\mathrm{c}}_{21}-\hat{\bar{c}}_{21}\right) \hat{\mathrm{z}}_{2}+\overline{\mathrm{c}}_{22} \mathrm{x}_{4}+\left(\overline{\mathrm{c}}_{22}-\hat{\mathrm{c}}_{22}\right) \widehat{\mathrm{z}}_{4}+\overline{\phi_{2}}-\hat{\phi}_{2}+\bar{\tau}_{2}-\hat{\bar{\tau}}
\end{aligned}
$$

By using the properties 4) and 5), it can be proved that the error of estimated parameter $\mathrm{w}_{1}$ and $\mathrm{w}_{2}$ are bounded.

The switching gain $k_{1}, k_{2}$ is chosen as a bound on the steady state estimation error on $x_{1}, x_{3}$, and $k_{3}, k_{4}$ is chosen to be larger than the error of estimated parameter $\mathrm{w}_{1}$ and $\mathrm{w}_{2}$. The chosen constants are:

$$
\mathrm{k}_{1}=0.1, \mathrm{k}_{2}=0.1, \mathrm{k}_{3}=1, \mathrm{k}_{4}=1
$$

The true system is tracking the desired trajectories by feedback linearization control law. The simulations were run using SIMNON, on two different initial conditions. The first set of initial conditions of the states and the states of estimation are all zeros. The simulation results for this set of initial conditions are shown in Fig. 5. It is clear that the sliding observer is working properly.

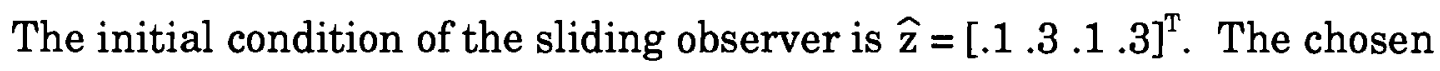


linear correction coefficient is $\mathrm{H}=\left[\begin{array}{llll}4 & 4 & 12 & 40\end{array}\right]$. The bound of disturbance is known as $\mathrm{k}_{1}: .5$ and $\mathrm{k}_{3}: 1$. The simulated results of the design algorithm SOON is -.46 and -.48 so that the chosen switching coefficients are $K=[.21$ $.252]$.
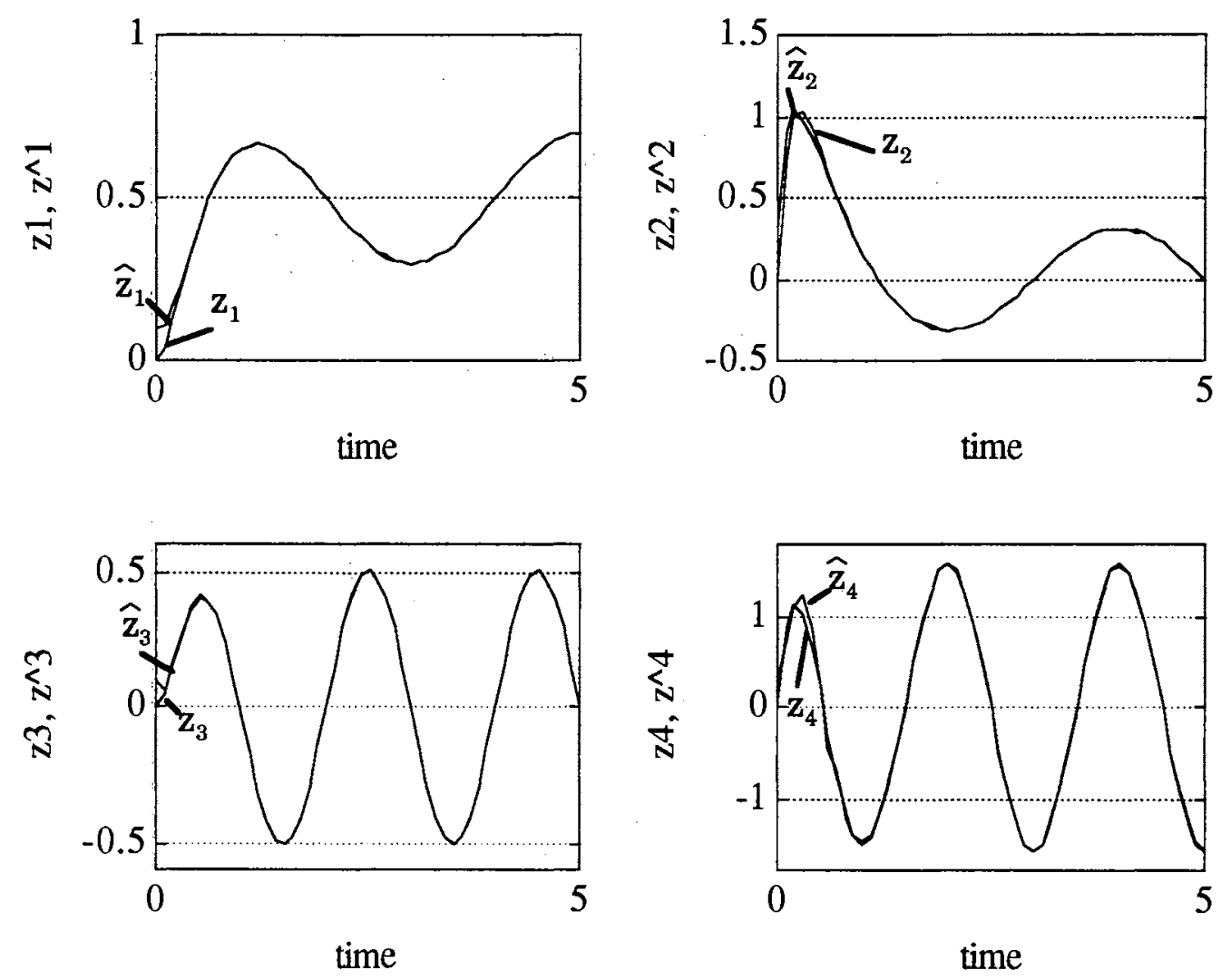

Figure 5.2 The simulated results of the sliding observer

\subsubsection{Feedback Linearization Control}

If the parameters are known exactly, the inverse dynamics control law (i.e. the Computed Torque control law) cancels exactly all of the nonlinear terms in (5.3) so that the closed loop system is linear and decoupled

$$
\mathrm{D}(\mathrm{q}) \ddot{\mathrm{q}}+\mathrm{C}(\mathrm{q}, \dot{\mathrm{q}}) \dot{\mathrm{q}}+\mathrm{g}(\mathrm{q})=\tau
$$


If the torque is $\tau=\mathrm{D}(\mathrm{q}) \nu+\mathrm{C}(\mathrm{q}, \dot{\mathrm{q}}) \dot{\mathrm{q}}+\mathrm{g}(\mathrm{q})$

Then, from (5.14) and (5.3), the equation of motion is

$$
\mathrm{D}(\mathrm{q})(\ddot{\mathrm{q}}-\mathrm{v})=0
$$

By property $1, \ddot{\mathrm{q}}=v$

We now consider the case when the manipulator is actually required to follow a desired trajectory, rather than merely reach a desired position. Note that a trajectory control problem may arise even when the task is merely to move a load from its initial position to a final position. The simple P.D. controller cannot be expected to handle the dynamic demands of trajectory tracking effectively. We consider first the use of feedback linearization. We then discuss the extension of adaptive control and sliding control based on the sliding observer.

The term $v$ is the interpretation of an outer loop control law with units of acceleration, which is typically chosen as:

$$
v=\ddot{q}_{d}-K_{\mathrm{v}} \dot{\widetilde{q}}-K_{p} \tilde{q}
$$

with $\widetilde{q}=q-q_{d}$, where $q_{d}(t)$; n-order vector of desired joint trajectories.

By plugging (5.17) into (5.16), we get a linear error dynamics

$$
\ddot{\widetilde{\mathrm{q}}}+\mathrm{K}_{\mathrm{v}} \dot{\widetilde{\mathrm{q}}}+\mathrm{K}_{\mathrm{p}} \widetilde{\mathrm{q}}=0
$$

If the gain matrices $K_{v}$ and $K_{p}$ are chosen as diagonal matrices with positive diagonal elements, then the closed-loop system is linear, decoupled, and exponentially stable. The global stability for this scheme is thus obvious. In fact, the closed-loop damping ratio and natural frequency may be arbitrarily assigned.

The Feedback Linearization Control based on Sliding Observer. The only difference between previous simulations and this section FL-SO is the 
control law in which the actual states are replaced by the sliding observer estimated states. The first set of initial conditions of the states and the states of the sliding observer are all zeros. The simulation for this set of initial conditions are shown in Fig. 5.9. It is clear that the FL-SO closed loop is stable. The initial condition of the sliding observer is $\hat{z}=\left[\begin{array}{lll}1 & 3 & 3 \\ \text {. } & .3 .3\end{array}\right]^{\mathrm{T}}$. The chosen linear correction coefficient is $\mathrm{H}=\left[\begin{array}{llll}6 & 9 & 16 & 60\end{array}\right]$. The bound of disturbance is known as $k_{1} .5$ and $k_{3}: 1$. The simulated results of the design algorithm SOON is -.58 and -.56 so that the chosen switching coefficients are $\mathrm{K}=\left[\begin{array}{llll}3 & 2 & .3 & 3\end{array}\right]$.
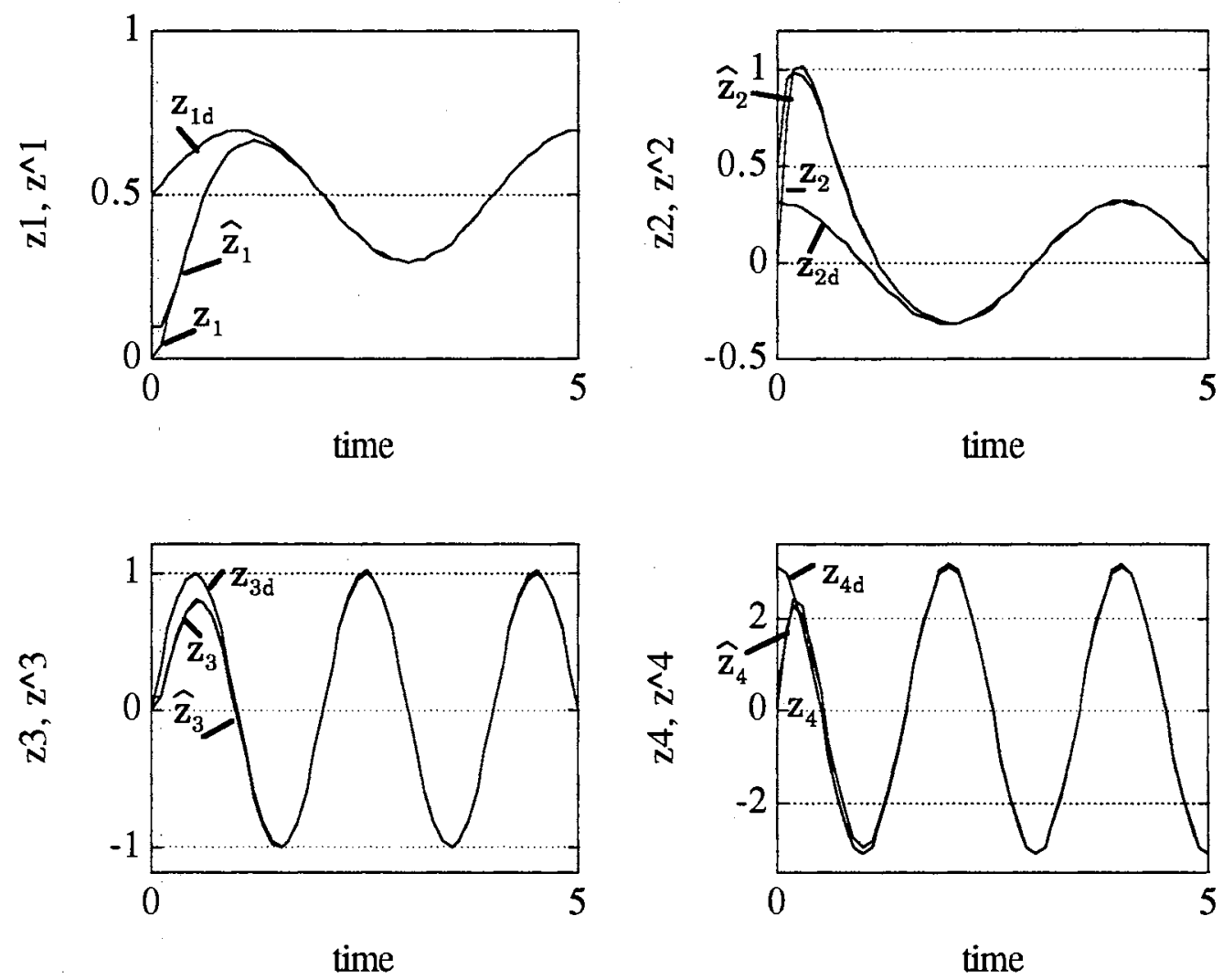

Figure 5.3 The simulated results of feedback linearization control based on the sliding observer 


\subsection{The Case with Parameter Uncertainty}

\subsubsection{The Design of the Sliding Observer}

Each angular position in a two-link manipulator is measured and both the velocity as well as the position are estimated. For an estimation scheme, we can intuitively include available nonlinear model to make estimation error small. On the contrary, however, it will take more time to compute and it is not desirable for our purpose. Therefore, although the nonlinear model with no uncertainty is available, we may adopt only linear terms for a fast estimation.

The simplified model for a sliding observer design is obtained by taking only linear terms. We need to notice that the input torque is not used and not measured.

$$
\begin{aligned}
& \dot{\mathrm{z}}_{1}=\mathrm{z}_{2} \\
& \dot{\mathrm{z}}_{2}=\mathrm{w}_{1} \\
& \dot{\mathrm{z}}_{3}=\mathrm{z}_{4} \\
& \dot{\mathrm{z}}_{4}=\mathrm{w}_{2}
\end{aligned}
$$

The equation (5.19) is really simple compared to the original equation. By comparing equations (5.10) and (5.13), one can identify the disturbance terms $\mathrm{w}_{1}$ and $\mathrm{w}_{2}$, as in (5.12), and try to find the bound for them. The bound of $w_{1}$ and $w_{2}$ are function of states and cannot be computed explicitly.

Therefore, the bounds can be assumed as the simulated maximum value of $\dot{\mathbf{x}}_{2}$ and $\dot{\mathrm{x}}_{4}$ of the operating range and the initial condition. Possibly, the bound can be violated during the transient for the different initial condition.

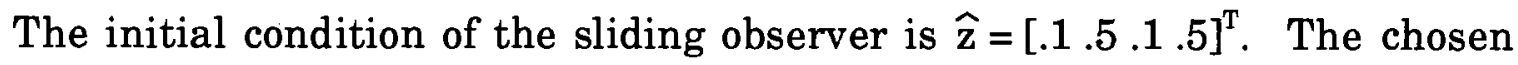

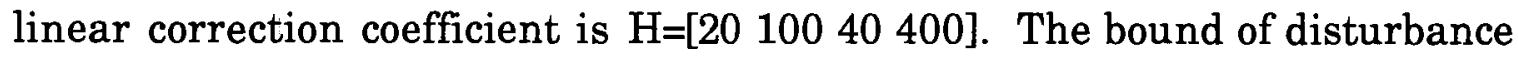


is known as $k_{1} .5$ and $k_{3}: 1$. The simulated results of the design algorithm SOON is -.65 and -.9 so that the chosen switching coefficients are $K=[.325$ $.4510]$.
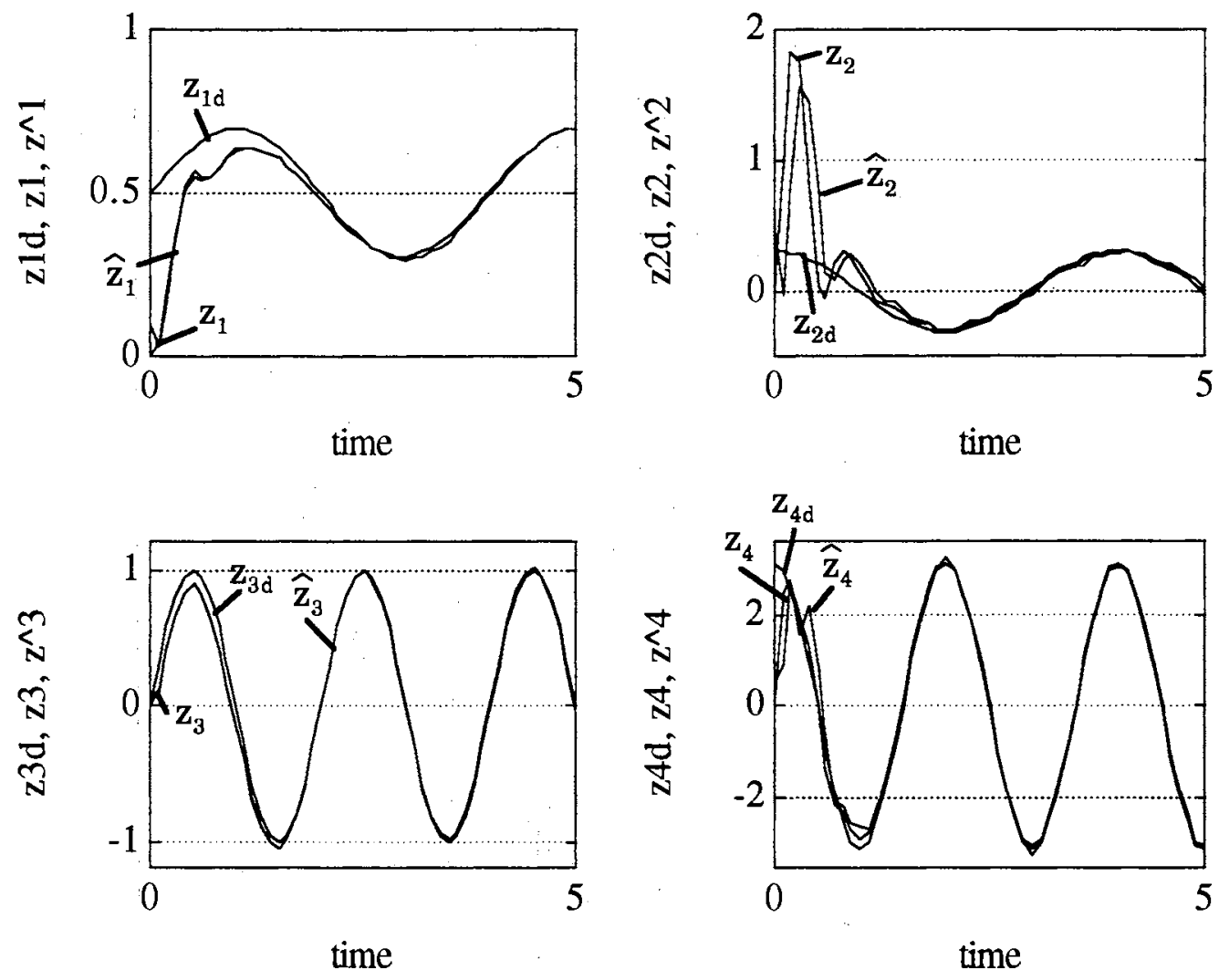

Figure 5.4 The simulated results of feedback linearization control based on the sliding observer with uncertain parameter

\subsubsection{Adaptive Control}

The Adaptive implementation of the inverse dynamics control law [Astrom, 1989; Slotine, 1991] is obtained by replacing D, C, and $\mathrm{g}$ by their estimates, i.e., 


$$
\tau=\widehat{D}(q)\left(\ddot{q}^{d}-K_{v} \dot{\widetilde{q}}-K_{p} \tilde{q}\right)+\widehat{C}(q, \dot{q}) \dot{q}+\widehat{g}(q)
$$

$\widehat{\mathrm{D}}, \widehat{\mathrm{C}}, \widehat{\mathrm{g}}$ have the same function form as $\mathrm{D}, \mathrm{C}$, $\mathrm{g}$ with estimated parameter $\theta_{1}, \ldots, \theta_{9}$

$$
\widehat{D} \ddot{q}+\widehat{C} \dot{q}+\widehat{g}=Y(q, \dot{q}, \ddot{q}) \hat{\theta}
$$

substitute (5.19) into (5.3), we got

$$
\mathrm{D} \ddot{\mathrm{q}}+\mathrm{C} \dot{\mathrm{q}}+\mathrm{g}=\widehat{\mathrm{D}}\left(\ddot{\mathrm{q}}^{\mathrm{d}}-\mathrm{K}_{\mathrm{v}} \dot{\tilde{\mathrm{q}}}-\mathrm{K}_{\mathrm{p}} \tilde{\mathrm{q}}\right)+\widehat{\mathrm{C}} \dot{\mathrm{q}}+\widehat{\mathrm{g}}
$$

Rearrange the terms as:

$$
\begin{gathered}
D \ddot{q}-\widehat{D} \ddot{q}_{d}+\widehat{D}\left(K_{v} \dot{\widetilde{q}}+K_{p} \widetilde{q}\right)=(\widehat{C}-C) \dot{q}+\widehat{g}-g \\
\left(\widehat{D} \ddot{q}-\hat{D} \ddot{q}^{d}\right)-(\hat{D} \ddot{q}-D \ddot{q})+\hat{D}\left(K_{v} \dot{\widetilde{q}}+K_{p} \widetilde{q}\right)=(\widehat{C}-C) q+(\widehat{g}-g)
\end{gathered}
$$

Let $(\tilde{\tilde{c}}):=(\hat{.})-($.

$$
\begin{aligned}
& \hat{\mathrm{D}}\left(\ddot{\widetilde{\mathrm{q}}}+\mathrm{K}_{\mathrm{v}} \dot{\widetilde{\mathrm{q}}}+\mathrm{K}_{\mathrm{p}} \tilde{\mathrm{q}}\right)=\widetilde{\mathrm{D}} \ddot{\mathrm{q}}+\widetilde{\mathrm{C}} \dot{\mathrm{q}}+\widetilde{\mathrm{g}}=\mathrm{Y}(\mathrm{q}, \dot{\mathrm{q}}, \ddot{\mathrm{q}}) \tilde{\theta} \\
& \dot{\tilde{\mathrm{q}}}+\mathrm{K}_{\mathrm{v}} \dot{\tilde{\mathrm{q}}}+\mathrm{K}_{\mathrm{p}} \tilde{\mathrm{q}}=\hat{\mathrm{D}}^{-1} \mathrm{Y} \tilde{\theta}:=\Phi \tilde{\theta}
\end{aligned}
$$

Rewrite (5.22) in state space form:

$$
\dot{\mathrm{z}}=\mathrm{A} \mathrm{z}+\mathrm{B} \Phi \theta
$$

A; Hurwitz matrix:

$$
A=\left[\begin{array}{cc}
0 & I \\
-K_{p} & -K_{v}
\end{array}\right] ; \text { and } B=\left[\begin{array}{l}
0 \\
I
\end{array}\right] ; z=\left[\begin{array}{c}
\tilde{\mathrm{q}} \\
\dot{\tilde{q}}
\end{array}\right]
$$

$\ddot{\mathrm{q}}$; measurable, $\hat{\mathrm{D}}^{-1}$; bounded.

The Update Law

$$
\dot{\tilde{\theta}}=-\Gamma^{-1} \Phi^{\mathrm{T}} \mathrm{B}^{\mathrm{T}} \mathrm{Pz}
$$


where $\Gamma=\Gamma^{T}>0$ and $\mathrm{P}$ is the unique symmetric positive definite solution to the Lyapunov equation:

$$
A^{T} P+P A+Q=0
$$

for a given symmetric, positive definite Q. Under these conditions then, the solution $\mathrm{x}$ of (5.23) satisfies:

$$
\mathrm{z} \Rightarrow 0 \text { as } \mathrm{T} \Rightarrow \infty
$$

with all signals remaining bounded.
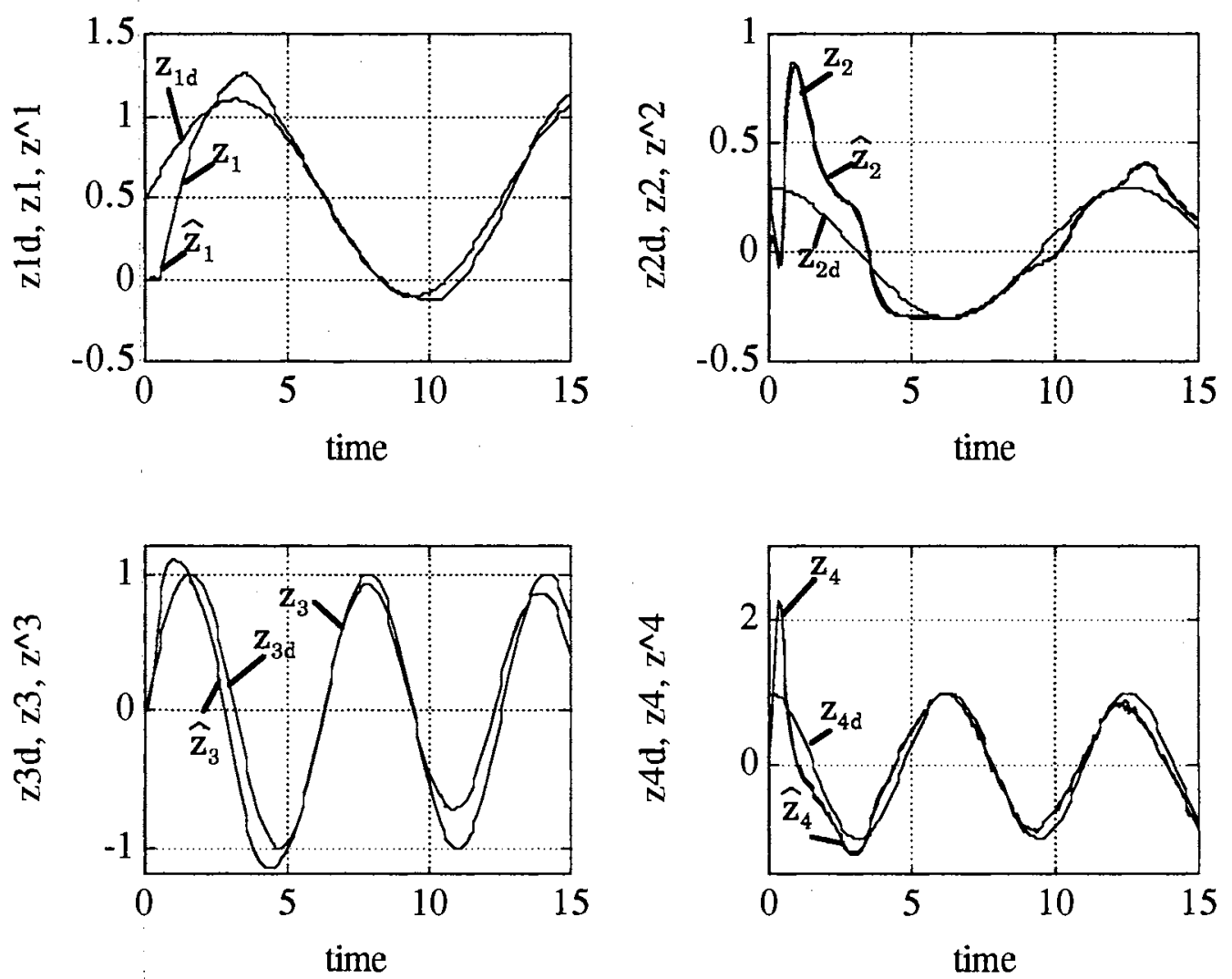

Figure 5.5 The simulated results of adaptive control based on the sliding observer with uncertain parameter 
Proof) Choose the Lyapunov function candidate [Astrom, 1989]:

$$
\begin{aligned}
\mathrm{V} & =\mathrm{z}^{\mathrm{T}} \mathrm{P} z+\tilde{\theta}^{\mathrm{T}} \Gamma \tilde{\theta} \\
\dot{\mathrm{V}} & =-\mathrm{z}^{\mathrm{T}} \mathrm{Q} z+2 \tilde{\sim}^{\mathrm{T}}\left[\Phi^{\mathrm{T}} \mathrm{B}^{\mathrm{T}} \mathrm{P} z+\Gamma \dot{\tilde{\theta}}\right]
\end{aligned}
$$

Using (5.25)

$$
\dot{\mathrm{V}}=-\mathrm{z}^{\mathrm{T}} \mathrm{Q} \mathrm{z} \leq 0
$$

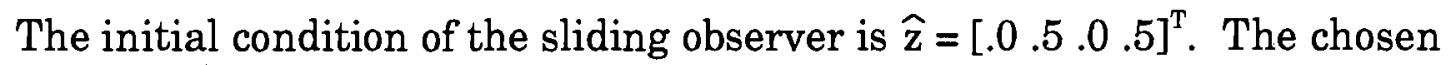
linear correction coefficient is $\mathrm{H}=\left[\begin{array}{l}4040060900\end{array}\right]$. The bound of disturbance is known as $k_{1} .5$ and $k_{3}: 1$. The simulated results of the design algorithm SOON is -.62 and -.9 so that the chosen switching coefficients are $K=[.315$ $.4510]$.

\subsubsection{Sliding Control}

We need to handle the model uncertainty or disturbance that leads to develop a sliding control and an adaptive control etc. The sliding control is one of the simple approaches to a robust control. It is easy to show that a perfect performance can be achieved theoretically in the presence of arbitrary uncertainty and disturbances.

Let the tracking error vector $\widetilde{q}=q-q^{d}$.

The sliding condition is:

$$
\frac{1}{2} \frac{\mathrm{d}}{\mathrm{dt}} \mathrm{s}_{\mathrm{i}}{ }^{2} \leq-\eta_{\mathrm{i}}\left|\mathrm{s}_{\mathrm{i}}\right| \quad\left(\eta_{\mathrm{i}}>0\right)
$$

where $\dot{\mathrm{s}}_{\mathrm{i}}=\dot{\tilde{\mathrm{q}}}_{\mathrm{i}}+\lambda_{\mathrm{i}} \widetilde{\mathrm{q}}_{\mathrm{i}}$

One may extend the sliding observer in a multi-variable case .

$$
\mathrm{s}=\dot{\widetilde{\mathrm{q}}}+\Lambda \widetilde{\mathrm{q}}=\dot{\mathrm{q}}-\dot{\mathrm{q}}_{\mathrm{r}}
$$


where $\dot{\mathrm{q}}_{\mathrm{r}}=\dot{\mathrm{q}}_{\mathrm{d}}-\Lambda \widetilde{\mathrm{q}} \quad\left(\mathrm{q}_{\mathrm{r}}\right.$ : Reference velocity $)$

The detail derivation of a sliding control law for a multi-variable case is shown by Slotine (1991).

Let us define a Lyapunov function candidate as:

$$
\mathrm{V}(\mathrm{t})=\frac{1}{2}\left[\mathrm{~s}^{\mathrm{T}} \mathrm{H} \mathrm{s}\right]
$$

Differentiating

$$
\dot{\mathrm{V}}(\mathrm{t})=\mathrm{s}^{\mathrm{T}}\left(\mathrm{H} \ddot{\mathrm{q}}-\mathrm{H} \ddot{\mathrm{q}}_{\mathrm{r}}\right)+\frac{1}{2} \mathrm{~s}^{\mathrm{T}} \dot{\mathrm{H}} \mathrm{s}
$$

From the system dynamics,

$$
\mathrm{H} \ddot{\mathrm{q}}=\tau-\mathrm{C} \dot{\mathrm{q}}-\mathrm{g}=\tau-\mathrm{C}\left(\mathrm{s}+\dot{\mathrm{q}}_{\mathrm{r}}\right)-\mathrm{g}
$$

plug in and get

$$
\mathrm{V}(\mathrm{t})=\mathrm{s}^{\mathrm{T}}\left(\tau-\mathrm{H} \ddot{\mathrm{q}}_{\mathrm{r}}-\mathrm{C} \mathrm{q}_{\mathrm{r}}-\mathrm{g}\right)
$$

The control input has the form as:

$$
\tau=\hat{\tau}-\mathrm{k} \operatorname{sgn}(\mathrm{s})
$$

$\hat{\tau}$ is computed as:

$$
\hat{\tau}=\widehat{H} \ddot{q}_{r}+\widehat{C} \dot{q}_{r}+\widehat{g}
$$

The components of the vector $\mathbf{k}$ will be chosen as:

$$
k_{i} \geq\left|\left[\widetilde{H}(q) q_{r}+\widetilde{C}(q, \dot{q}) \dot{q}_{r}+\widetilde{g}(q)\right]_{i}\right|+\eta_{i}
$$

so that

$$
\dot{\mathrm{V}} \leq-\sum_{\mathrm{i}=1}^{\mathrm{n}} \eta_{\mathrm{j}}\left|\mathrm{s}_{\mathrm{i}}\right|
$$

As in the single-input case, this sliding condition makes the state reach within a finite time and remain on the surface.

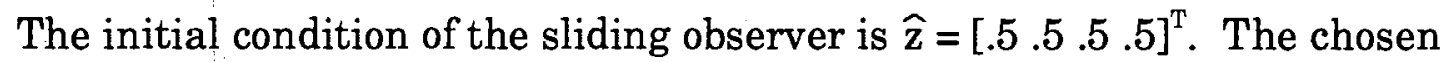


linear correction coefficient is $\mathrm{H}=\left[\begin{array}{l}40 \\ 40060900\end{array}\right]$. The bound of disturbance is known as $k_{1} .5$ and $k_{3}: 1$. The simulated results of the design algorithm SOON is -.62 and -.9 so that the chosen switching coefficients are $K=[.315$ $.4510]$.
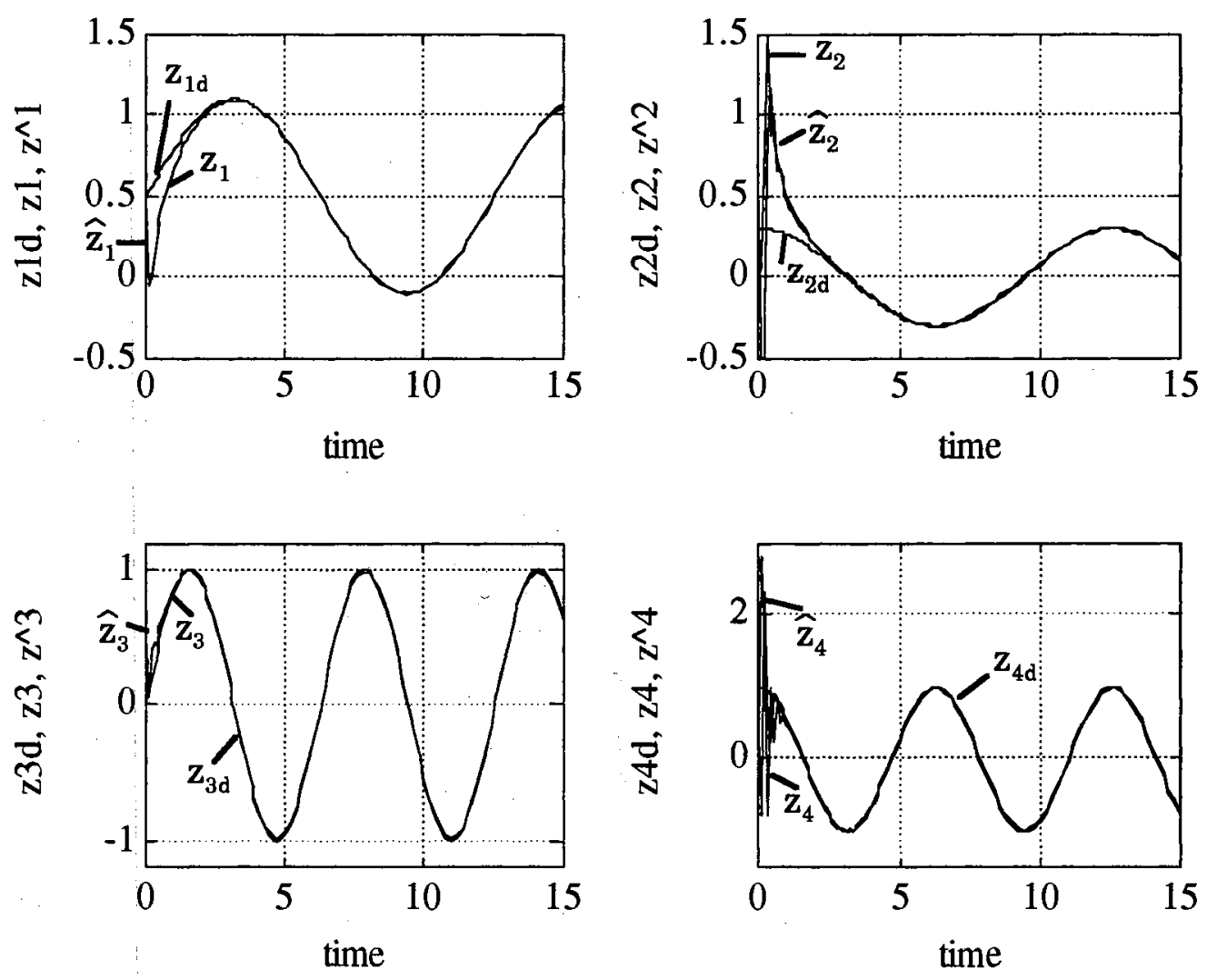

Figure 5.6 The simulated results of sliding control based on the sliding observer with uncertain parameter 


\section{CHAPTER V I}

\section{STOCHASTIC SLIDING OBSERVER DESIGN}

\subsection{Introduction}

Optimal estimators that minimize the estimation error in a well-defined statistical sense, are of particular interest. For the linear filtering problem, under the assumption that the process noise and the nonsingular measurement noise are white, the Kalman-Bucy filter [Kalman, 1961; Kalman, 1960] is optimal in the sense of the mean-square estimation error criterion. For realizing the filter, we should know the exact intensities of noise which compose the state error covariance matrix, i.e. the Riccati equation. Practically, however, the noises are not measurable and the noise intensities may change according to variation of the operating condition.

To avoid difficulty in adapting a filter in accordance with variation of noise characteristics, Drakunov, in 1983, suggested an Adaptive Quasioptimal Filter [Drakunov, 1986] which is insensitive to inexact knowledge of the noise intensity. By using the averaging theory his paper shows that the Adaptive Quasioptimal filter, which is actually a sliding observer, can be robust against the changed measurement noise characteristics. With the known statistical properties of the sensor noises, Misawa applied the methods of statistical linearization (the describing function technique [Gelb, 1968]) in designing the stochastic sliding observer.

If all the uncertainties in the input noise can be modeled as white noises, 
then one can use the Random Input Describing Function (RIDF) in the design process. Compared to the Drakunov's analysis, Misawa [Misawa, 1988] included the linear gain " $\mathrm{H} \mathrm{C} \mathrm{z"} \mathrm{in} \mathrm{his} \mathrm{analysis} \mathrm{for} \mathrm{a} \mathrm{first} \mathrm{order}$ system.

In this preliminary study, the robustness analysis for a second order system was studied about the effect of changing in noise characteristics and parameter mismatch. The theoretical prediction of a steady state estimation error covariance of the sliding observer using RIDF shows good agreements with the simulation, as well as with the first order case of Misawa [ 1988].

\subsection{Sliding Observer Design for Noisy Measurements}

Misawa's method [1988] for the sliding observer design procedure for noisy measurement is utilized. System and measurement equations are

$$
\begin{aligned}
& \dot{z}=A z+w \\
& y=C z+v
\end{aligned}
$$

where $\mathrm{w}$ and $\mathrm{v}$ are assumed to be stationary and independent white noise.

The sliding observer structure:

$$
\dot{\hat{z}}=A \hat{z}+H(y-C \widehat{z})+K 1_{s}(\widetilde{y})
$$

where $\tilde{\mathbf{y}}=\mathrm{y}-\hat{\mathbf{y}}$

The estimation error dynamics for the sliding observer:

$$
\begin{aligned}
& \dot{\mathrm{x}}=(\mathrm{A}-\mathrm{H} \mathrm{C}) \mathrm{x}-\mathrm{K} 1_{\mathrm{s}}(\widetilde{\mathrm{y}})+\mathrm{w}-\mathrm{H} \mathrm{v} \\
& 1_{\mathrm{s}}^{\mathrm{T}}(\widetilde{\mathrm{y}})=\left[\operatorname{sign}\left(\widetilde{\mathrm{y}}_{1}\right), \operatorname{sign}\left(\widetilde{\mathrm{y}}_{2}\right), \ldots, \operatorname{sign}\left(\widetilde{\mathrm{y}}_{\mathrm{m}}\right)\right]
\end{aligned}
$$


The switching function can be approximated by the RIDF function [Gelb, 1974]:

$$
1_{s}(\widetilde{y}) \cong N_{1} \widetilde{y}=N_{1}(C x+v)
$$

where $N_{1}$ is the $m \times m$ matrix of RIDF of $1_{s}$ and the function of statistics of $\widetilde{\mathbf{y}}$. By assuming $\widetilde{\mathrm{y}}$ is a zero mean Gaussian Process, $\mathrm{N}_{1}$ is determined by covariance matrix of $\widetilde{y}$. The estimation error dynamics can be rewritten as:

$$
\begin{aligned}
\dot{x} & =(A-H C) x-K N_{1}(C x+v)+w-H v \\
& =A x-\left(H+K N_{1}\right) C x-\left(H+K N_{1}\right) v+w
\end{aligned}
$$

Let $\mathrm{H}^{*}=\mathrm{H}+\mathrm{KN}_{1}$

$$
\begin{aligned}
\dot{\mathrm{x}} & =\mathrm{A} \mathrm{x}-\left(\mathrm{H}+\mathrm{KN}_{1}\right)(\mathrm{Cx}+\mathrm{v})+\mathrm{w} \\
& =\mathrm{A} \mathrm{x}-\mathrm{H}^{*}(\mathrm{y}-\mathrm{C} \widehat{\mathrm{z}})+\mathrm{w}
\end{aligned}
$$

The lumped gain matrix $\mathrm{H}^{*}$ is also a function of the covariance matrix of $\tilde{y}$.

$$
\begin{aligned}
& \widetilde{y}=C x+v \\
& \begin{aligned}
E\left[\widetilde{y}(t) \widetilde{y}^{T}(t)\right]=C E\left[x x^{T}\right] C^{T}+E\left[v v^{T}\right] \\
=C P C^{T}+R
\end{aligned}
\end{aligned}
$$

Considering the measurement noise is correlated, the gain matrix $\mathrm{H}^{*}$ should be determined by an iterative optimization method.

The plant is assumed to be described as

$$
\begin{aligned}
& \dot{\mathrm{z}}=\mathrm{Az}+\mathrm{w} \\
& \mathrm{y}=\mathrm{C} \mathrm{z}+\mathrm{v}
\end{aligned}
$$

where $E\left[w(t) w(t+\tau)^{r}\right]=Q \delta(\tau)$ and $w$ is a $n$-order column vector that 
includes all nonlinearities and uncertainties. Suppose that the measurement noise is colored, i.e. it is correlated. It is assumed that the measurement noise can be modeled through the use of a "shaping filter":

$$
\dot{\mathrm{v}}=\mathrm{E} \mathrm{v}+\mathrm{F} \mathrm{v}_{1}
$$

where the matrix $\mathrm{E}$ and $\mathrm{F}$ are chosen properly and $\mathrm{v}_{1}$ is a white noise process:

$$
\mathrm{E}\left[\mathrm{v}_{1}(\mathrm{t}) \mathrm{v}_{1}(\mathrm{t}+\tau)^{\mathrm{T}}\right]=\mathrm{R} \delta(\tau)
$$

The augmented system including the noise model is:

$$
\dot{\mathrm{z}}=\left[\begin{array}{c}
\dot{\mathrm{z}} \\
\dot{\mathrm{v}}
\end{array}\right]=\left[\begin{array}{cc}
\mathrm{A} & 0 \\
0 & \mathrm{E}
\end{array}\right]\left[\begin{array}{l}
\mathrm{z} \\
\mathrm{v}
\end{array}\right]+\left[\begin{array}{cc}
\mathrm{D} & 0 \\
0 & \mathrm{~F}
\end{array}\right]\left[\begin{array}{c}
\eta \\
\mathrm{v}_{1}
\end{array}\right]
$$

where $w=D \eta$.

Using the lumped gain matrix in the RIDF method, the sliding observer can be written as:

$$
\dot{\widehat{z}}=A \hat{z}+H^{*}(y-C \hat{z})
$$

The augmented estimation error dynamics is:

$$
\dot{\mathrm{x}}=\left[\begin{array}{l}
\dot{\mathrm{x}} \\
\dot{\mathrm{v}}
\end{array}\right]=\left[\begin{array}{cc}
\mathrm{A}-\mathrm{H}^{*} \mathrm{C} & -\mathrm{H}^{*} \\
0 & \mathrm{E}
\end{array}\right]\left[\begin{array}{l}
\mathrm{x} \\
\mathrm{v}
\end{array}\right]+\left[\begin{array}{cc}
\mathrm{D} & 0 \\
0 & \mathrm{~F}
\end{array}\right]\left[\begin{array}{l}
\eta \\
\mathrm{v}_{1}
\end{array}\right]=\mathrm{A}_{\mathrm{m}} x+\mathrm{G}_{\mathrm{m}} w
$$

The covariance matrix of estimation error:

$$
P=\left[\begin{array}{ll}
E\left[\widetilde{\mathbf{x}} \widetilde{\mathbf{x}}^{T}\right] & E\left[\widetilde{\mathbf{x}} v^{T}\right] \\
E\left[v \widetilde{\mathbf{x}}^{T}\right] & E\left[v v^{T}\right]
\end{array}\right]
$$

The covariance propagation equation:

$$
\dot{\mathrm{P}}=A_{\mathrm{m}} \mathrm{P}+\mathrm{PA}_{\mathrm{m}}{ }^{\mathrm{T}}+\mathrm{G}_{\mathrm{m}} \Phi \mathrm{G}_{\mathrm{m}}{ }^{\mathrm{T}}
$$


where $\Phi=\operatorname{diag}(\mathrm{Q}, \mathrm{R})$.

Assuming that the original process is ergodic, the steady state Lyapunov equation can be solved for a fixed matrix $\mathrm{H}^{*}$.

Let the cost function:

$$
J=\operatorname{trace}\left(E\left[\begin{array}{ll}
x & \left.x^{T}\right]
\end{array}\right)=\operatorname{trace}\left(M P M^{T}\right)\right.
$$

where $M$ is the $n \times(n+m)$ matrix with $n \times n$ identity matrix and the remain is zero. To get the optimal gain $\mathrm{H}^{*}$ that minimizes the chosen cost function, the solution of Lyapunov equation should be obtained iteratively.

\subsection{1st Order System Example}

In this section, the former example of Misawa [Misawa, 1988] is summarized. Let us consider the first-order system with the correlated measurement noise that can be modeled as a first-order Gauss-Markov Process:

$$
\begin{aligned}
& \dot{\mathrm{z}}=-\mathrm{az}+\mathrm{w} \\
& \mathrm{y}=\mathrm{z}+\mathrm{v} \\
& \tau \dot{\mathrm{v}}=-\mathrm{v}+\mathrm{v}_{1}
\end{aligned}
$$

where $Q=q^{2}=E\left[w^{\top}\right]=1.0, R=r^{2}=E\left[v_{1} v_{1}^{\top}\right]=0.01, a=1, \tau=0.05$

The Kalman filter is optimal in the least-square sense:

$$
\dot{\hat{z}}=-\mathrm{a} \hat{\mathbf{z}}+\mathrm{h}(\mathrm{y}-\widehat{\mathbf{z}})
$$

Assuming the measurement noise $\mathrm{v}$ is white, we get the constant Kalman filter gain by using a standard CACSD software.

$$
\mathrm{h}=9.05 \Rightarrow \mathrm{KF} 1
$$

For the colored noise case, the covariance error propagation equation (6.12) should be solved to get the optimal gain $\mathrm{H}^{*}$. The first step in the 
design process would be to get the statistical steady state variance of estimation error. For the 1st order system, it can be solved explicitly.

$$
\mathrm{P}_{\mathrm{x}}=\mathrm{E}\left[\mathrm{x}^{2}\right]=\frac{\mathrm{q}^{2}[(\mathrm{a}+\mathrm{h}) \tau+1]+\mathrm{h}^{2} \mathrm{r}^{2}}{2(\mathrm{a}+\mathrm{h})[(\mathrm{a}+\mathrm{h}) \tau+1]}
$$

To find the gain $\mathrm{h}$ that will minimizes $\mathrm{P}_{\tilde{\mathbf{x}}}$ :

$$
\begin{aligned}
& \frac{\partial p_{x}}{\partial h}=0 \\
& \Rightarrow h^{*}=\frac{(1+a t)\left(t q^{2}-a^{2} r^{2} \pm \sqrt{a^{2} r^{4}+q^{2} r^{2}}\right)}{r^{2}(2 a t+1)-q^{2} r^{2}}=17.356
\end{aligned}
$$

For a first-order system, the sliding observer is

$$
\dot{\hat{z}}=-\mathrm{a} \hat{\mathbf{z}}+\mathrm{k} \operatorname{sign}(\mathbf{y}-\widehat{z})
$$

where the linear gain term of equation (6.2) is already stable so that no more terms need to guarantee the stability. Because the optimal gain $h^{*}$ is known, we can proceed to compute the gain for the sliding observer. For this case, the covariance of estimation error and noise are

$$
\begin{aligned}
& \mathrm{P}_{\mathrm{x}}=\mathrm{E}\left[\mathrm{x}^{2}\right]=0.07 \\
& \mathrm{P}_{\mathrm{y}}=\frac{\mathrm{r}^{2}}{2 \mathrm{t}}=0.1
\end{aligned}
$$

The covariance of $E\left[\widetilde{y}^{2}\right]$ is

$$
\begin{aligned}
& \sigma_{\tilde{y}}^{2}=\mathrm{E}\left[\tilde{\mathrm{y}}^{2}\right]=\mathrm{E}\left[(\mathrm{x}+\mathrm{v})^{2}\right] \\
& =\mathrm{E}\left[\mathrm{x}^{2}\right]+2 \mathrm{E}[\mathrm{xv}]+\mathrm{E}\left[\mathrm{v}^{2}\right] \\
& =0.07-0.0904+0.1=0.0796 \\
& \sigma_{\mathbf{y}}=0.282
\end{aligned}
$$

With this input statistics, the gain $\mathrm{k}$ for the sliding observer is 


$$
\begin{aligned}
& \mathrm{h}^{*}=\sqrt{\frac{2}{\pi}} \frac{\mathrm{k}}{\sigma_{\mathrm{y}}} \\
& \mathrm{k}_{\mathrm{so}}=\mathrm{h}^{*} \sigma_{\mathrm{y}} \sqrt{\frac{\pi}{2}}=6.13
\end{aligned}
$$

Using RIDF, the steady state covariance of the sliding observer for the 1st order example is

$$
\begin{aligned}
& \sigma_{\tilde{y}}^{2}=\frac{1}{2\left(a+\sqrt{\frac{2}{\pi}} \frac{k}{\sigma_{\tilde{y}}}\right)}\left[\frac{\left(a^{2}-\frac{1}{\tau^{2}}\right) \frac{r^{2}}{\tau}}{a+\frac{1}{\tau}+\sqrt{\frac{2}{\pi}} \frac{k}{\sigma_{\tilde{y}}}}+q^{2}+\frac{r^{2}}{\tau^{2}}\right] \\
& P_{s o}=E\left[x^{2}\right]=\sigma_{\tilde{y}}^{2}+\left(1-2 \frac{a+\frac{1}{\tau}}{a+\frac{1}{\tau}+\sqrt{\frac{2}{\pi}} \frac{k}{\sigma_{\tilde{y}}}}\right) \frac{r^{2}}{2 \tau}
\end{aligned}
$$

The above implicit equation for the output error covariance can be solved numerically.

\subsection{2nd Order System Example}

To extend the first order system to the second order system, consider a simple second-order system, with correlated measurement noise that can be modeled as a second-order Gauss-Markov Process:

$$
\begin{aligned}
& \dot{z}_{1}=z_{2} \\
& \dot{z}_{2}=-a_{1} z_{1}-a_{2} z_{2}+w \\
& y=z_{1}+v \\
& \tau \dot{v}=-v+v_{1}
\end{aligned}
$$

where $Q=q^{2}=E\left[w w^{\top}\right]=1.0, R=r^{2}=E\left[v_{1} v_{1}^{\top}\right]=0.01, a=1, \tau=0.05$

The Kalman filter is optimal in the least-square sense and has the form: 


$$
\hat{\mathrm{z}}=-\mathrm{a} \hat{\mathrm{z}}+\mathrm{h}(\mathrm{y}-\hat{\mathrm{z}})
$$

where $z=\left[\begin{array}{ll}z_{1} & z_{2}\end{array}\right]^{\top}$. Assuming the measurement noise $v$ is white, we get the constant Kalman filter gain by using a standard CACSD software.

$$
\mathrm{H}=\left[\begin{array}{l}
2.7010 \\
3.6478
\end{array}\right] \Rightarrow \mathrm{KF} 1
$$

For the colored noise case, the covariance error propagation equation (6.12) should be solved to get the optimal gain $\mathrm{H}^{*}$. The first step in the design process would be to get the statistical steady state variance of estimation error. For the 2nd order system, it may be convenient to solve numerically. To find the optimal gain matrix $\mathrm{H}^{*}$ that will minimize the trace of estimation error covariance matrix, the Lyapunov equation is solved iteratively. The covariance equation (6.12) is solved for the steady state by using MATLAB with the matrices:

$$
\begin{aligned}
A_{m} & =\left[\begin{array}{ccc}
-h_{1} & 1 & -h_{1} \\
a_{1}-h_{2} & a_{2} & -h_{2} \\
0 & 0 & E
\end{array}\right] \\
G_{m} & =\left[\begin{array}{cc}
D & 0 \\
0 & F
\end{array}\right]=\left[\begin{array}{ll}
0 & 0 \\
1 & 0 \\
0 & F
\end{array}\right] \text { and } \Phi=\left[\begin{array}{cc}
Q & 0 \\
0 & R
\end{array}\right]
\end{aligned}
$$

One can see the matrix $A_{m}$ is a function of $h_{1}$ and $h_{2}$ that is to be solved. The Gain matrix $\mathrm{H}^{*}$ is

$$
\mathrm{H}^{*}=\left[\begin{array}{l}
3.1790 \\
4.5289
\end{array}\right] \Rightarrow \mathrm{KF} 2
$$

For a second-order system, the sliding observer is

$$
\dot{\mathrm{z}}=-\mathrm{a} \hat{\mathrm{z}}+\mathrm{k} \operatorname{sign}(\mathrm{y}-\hat{\mathrm{z}})
$$

where $z=\left[\begin{array}{lll}z_{1} & z_{2}\end{array}\right]^{\top}$ and the linear gain term of equation (6.2) is not need to 
guarantee the stability. Because the optimal gain $\mathrm{H}^{*}$ is known, we proceed the compute the gain for the sliding observer. For this case, the covariance of $E\left[\widetilde{y}^{2}\right]$ is calculated from the solution of the steady state Lyapunov equation. With this input statistics, $\sigma_{\mathrm{y}}=0.3102$, the gain $\mathrm{K}$ for the sliding observer is

$$
\mathrm{K}=\left[\begin{array}{l}
1.2360 \\
1.7609
\end{array}\right] \Rightarrow \mathrm{SO}
$$

\subsection{Prediction and Simulation}

\subsubsection{Effect of Measurement Noise}

In order to test the performance robustness of the sliding observer, the effect of deviation of measurement noise intensity from the nominal value is investigated. When the noise intensities are the ones considered in the design process, the Kalman filter should be the optimal one for a linear system. In this case, the noise is assumed to be colored noise as the equation (6.7) so that the Kalman filter KF1 is not the optimal one and the Kalman filter KF2 is the optimal one at the nominal point.
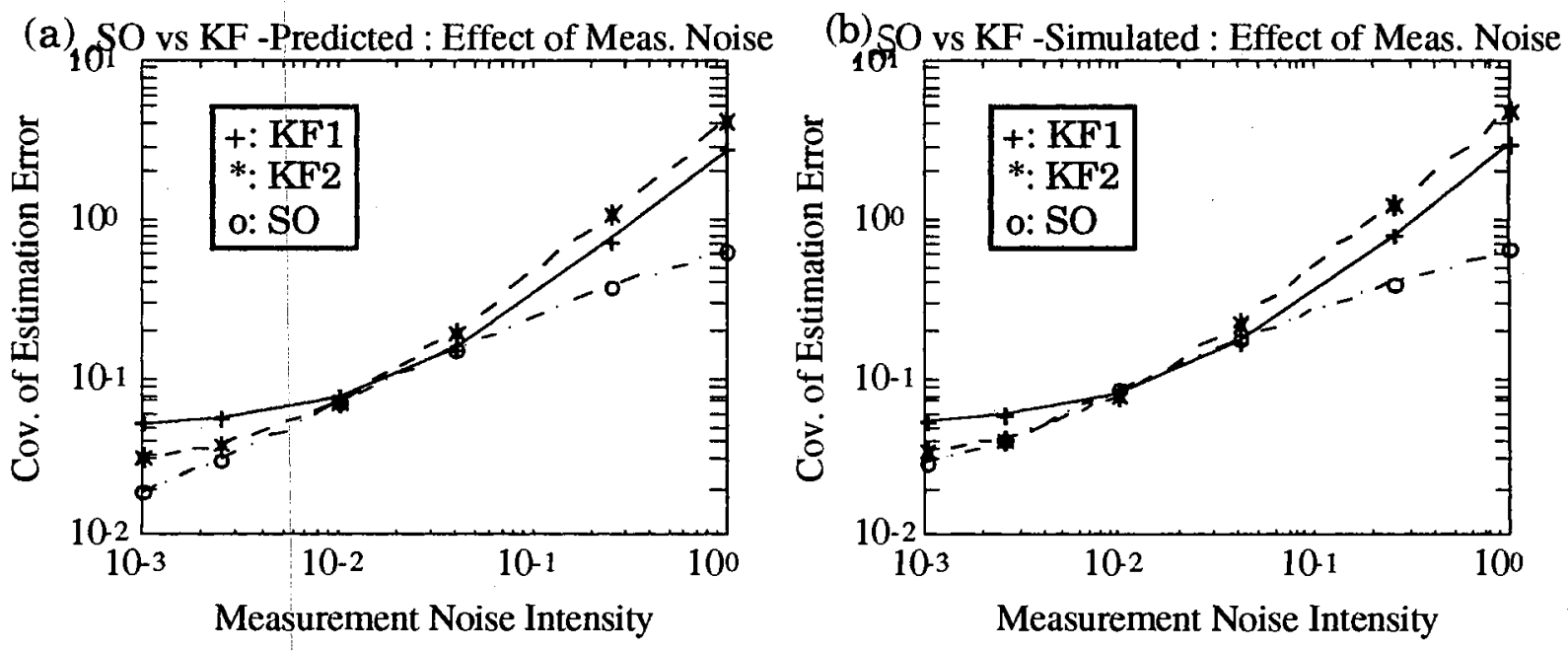

Figure 6.1 Effect of measurement noise: SO vs. KF (for the 1st order system)
a) Prediction
b) Simulated results 


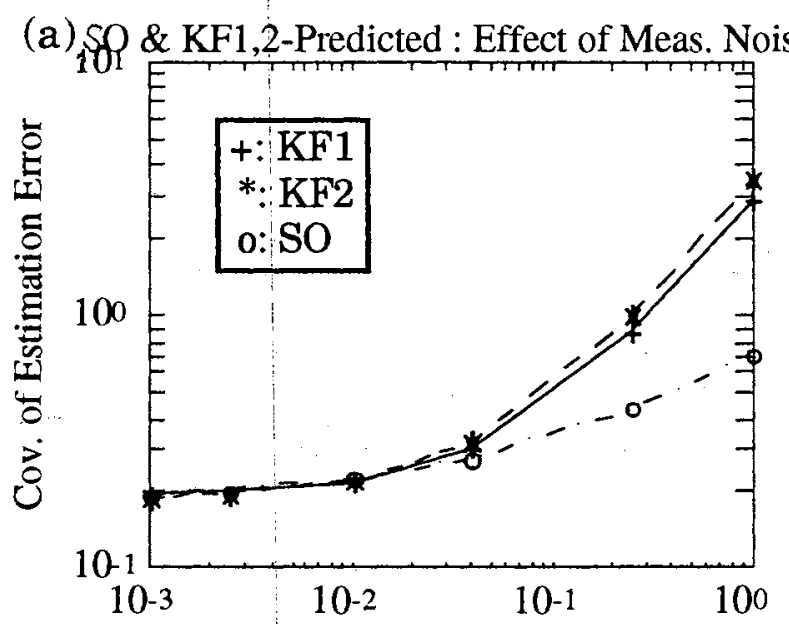

Meas. Noise Intensity - 2nd Order Sys.

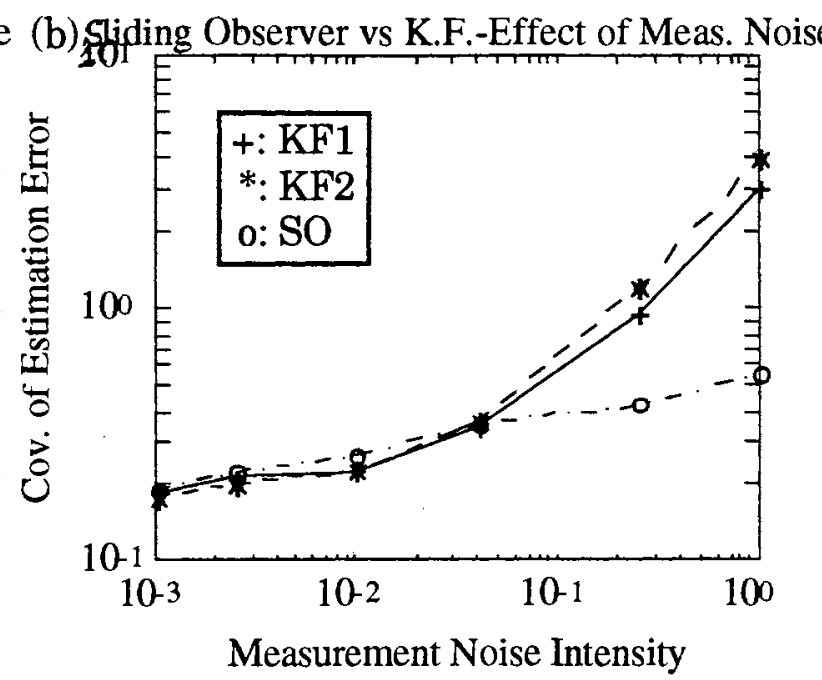

Figure 6.2 Effect of measurement noise: SO vs. KF (for the 2nd order system)

a) Prediction b) Simulated results

The predictions for 1st and 2nd order case are plotted in Figure 6.1 (a) and Figure 6:2 (a) and the Monte Carlo simulation that is the mean of the time averages over 100 simulations are shown in Figure $6.1(\mathrm{~b})$ and Figure 6.2 (b). Clearly, at the nominal point, the KF2 is the optimal one, seen both from the predicted values and from the values of simulation. The prediction of the SO at nominal point coincides with the KF2 for both cases. When the measurement noise intensity deviates from the nominal condition, the inherent robustness of the SO with respect to changes in the measurement noise becomes evident for both cases. In prediction results, for all values of measurement noise intensities are different from the nominal value, the sliding observer is the best one. On the contrary, the simulation shows the 1 st order SO is best for all values, but the 2nd order SO is not best. Even thought the 2nd order SO is not best for all values, it apparently shows robustness against the variation of measurement noise. 
This result is due to the fact that the sign function has an inherent adaptive-type of behavior with respect to changes in the measurement noise. This fact can be clearly observed if one recalls the definition of RIDF for the sign function:

$$
\operatorname{sign}(\widetilde{\mathbf{y}})=\sqrt{\frac{2}{\pi}} \frac{1}{\sigma_{\tilde{y}}}
$$

Since $\sigma_{\mathfrak{y}}$ is directly affected by the changes in the measurement noise, one can see that the increased noise reflects as a virtual decrease in the measurement noise intensity is reflected as virtual increase in the filter gain. This behavior is exactly the desired from an adaptive filter in order to maintain a good performance for a wide range of changes in the measurement noise intensity.

\subsubsection{Effect of Process Noise}

The robustness about the change of process noise was investigated. The predicted results for 1st and 2nd order systems are shown in Figure 6.3 (a) and Figure 6.4 (a). The Monte Carlo simulation that is the averaging the time averages of 10 seconds over 100 simulations are shown in Figure $6.3(\mathrm{~b})$ and Figure 6.4 (b).

In this case, the sliding observer is not profitable. For both prediction and simulation, the $\mathrm{KF} 1$ shows good robustness for smaller intensities and the KF2 shows a good robustness for larger intensities; it is probably due to the fact that for large process noise intensity a larger filter gain is required so that suitable corrections are provided to the filter. 

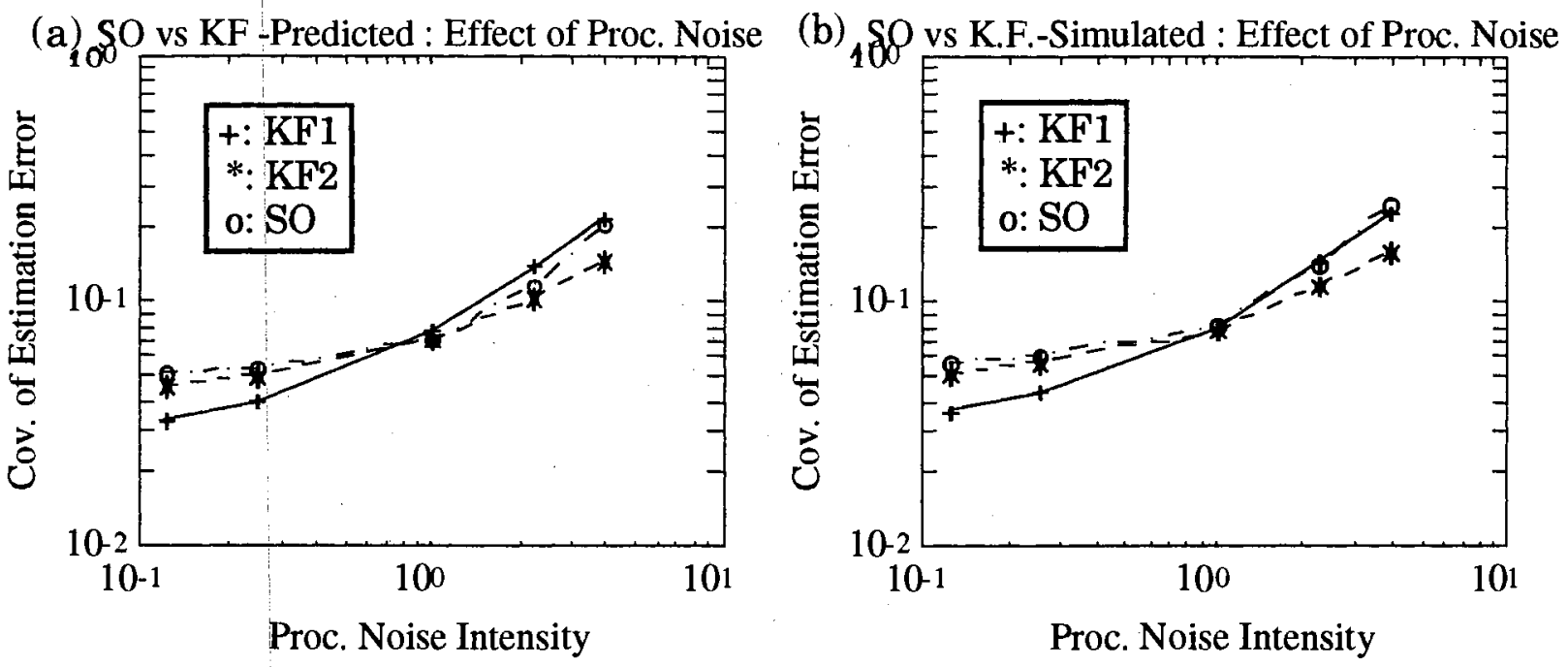

Figure 6.3 Effect of process noise: SO vs. KF (for the 1st order system)

a) Prediction b) Simulated results

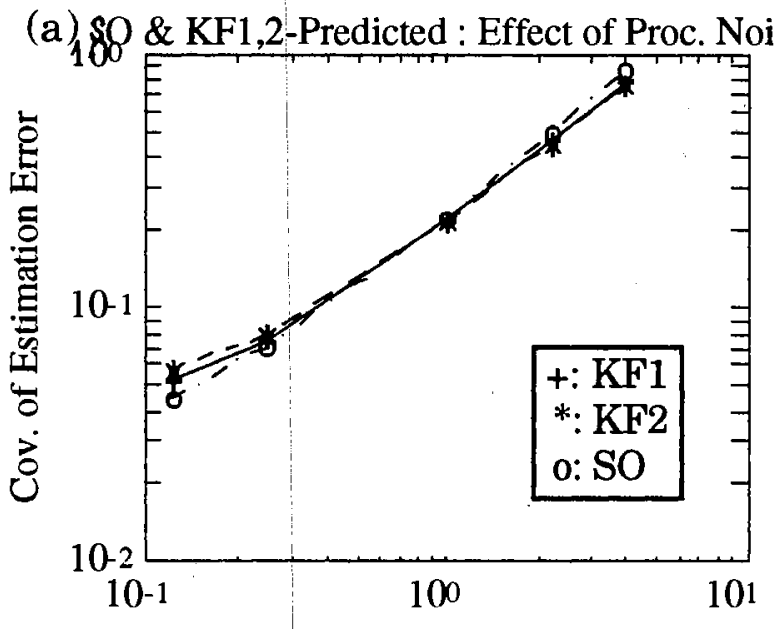

Proc. Noise Intensity - 2nd Order Sys.

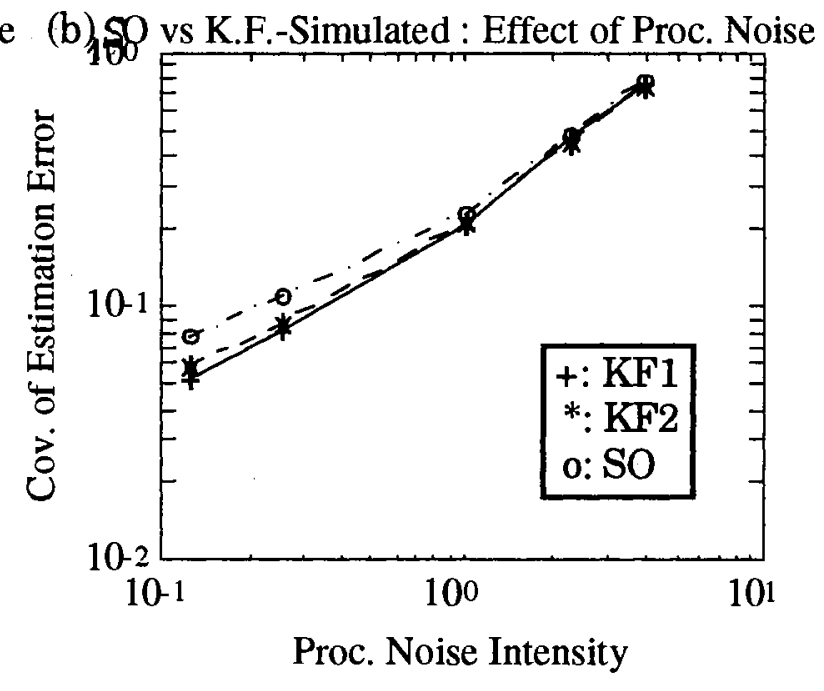

Figure 6.4 Effect of process noise: SO vs. KF (for the 2nd order system)

a) Prediction b) Simulated results 


\subsubsection{Effect of Parametric Mismatch}

System Equations:

$$
\begin{aligned}
\dot{z} & =A z+w \\
y & =C x+v \\
\hat{z} & =A^{0} \widehat{z}+H(y-C \widehat{z}) \\
= & A^{0} \widehat{z}+H(C(z-\hat{z})+v)
\end{aligned}
$$

The error dynamics:

$$
\dot{\mathrm{x}}=(\mathrm{A}-\mathrm{HC}) \mathrm{x}-\mathrm{H} v+\widetilde{\mathrm{A}} \widehat{z}+\mathrm{w}
$$

where $\widetilde{A}=A-A^{\circ}, A^{\circ}$ is nominal value and $A$ is a actual value.

The estimation error covariance matrix is

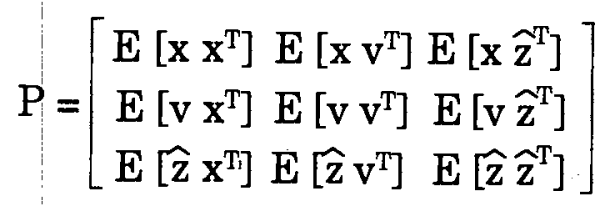

It can be propagated as:

$$
\text { where } A_{p}=\left[\begin{array}{ccc}
A-H C & -H & \widetilde{A} \\
0 & E & 0 \\
H C & H & A^{\circ}
\end{array}\right] \text { and } G_{p}=\left[\begin{array}{cc}
D & 0 \\
0 & F \\
0 & 0
\end{array}\right]
$$

The steady state error covariance $P$ is obtained by solving the Lyapunov equation with $\dot{\mathrm{P}}$ set to zero. For 2 nd order system, the matrix $A_{\mathrm{p}}$ is a $5 \times 5$ matrix and a function of $\mathrm{H}$ with given parameter $\mathrm{A}$. The optimal solution of Lyapunov equation can be solved numerically, for example, by the steep decent method. The remain procedure for prediction is the same as the previous cases. 

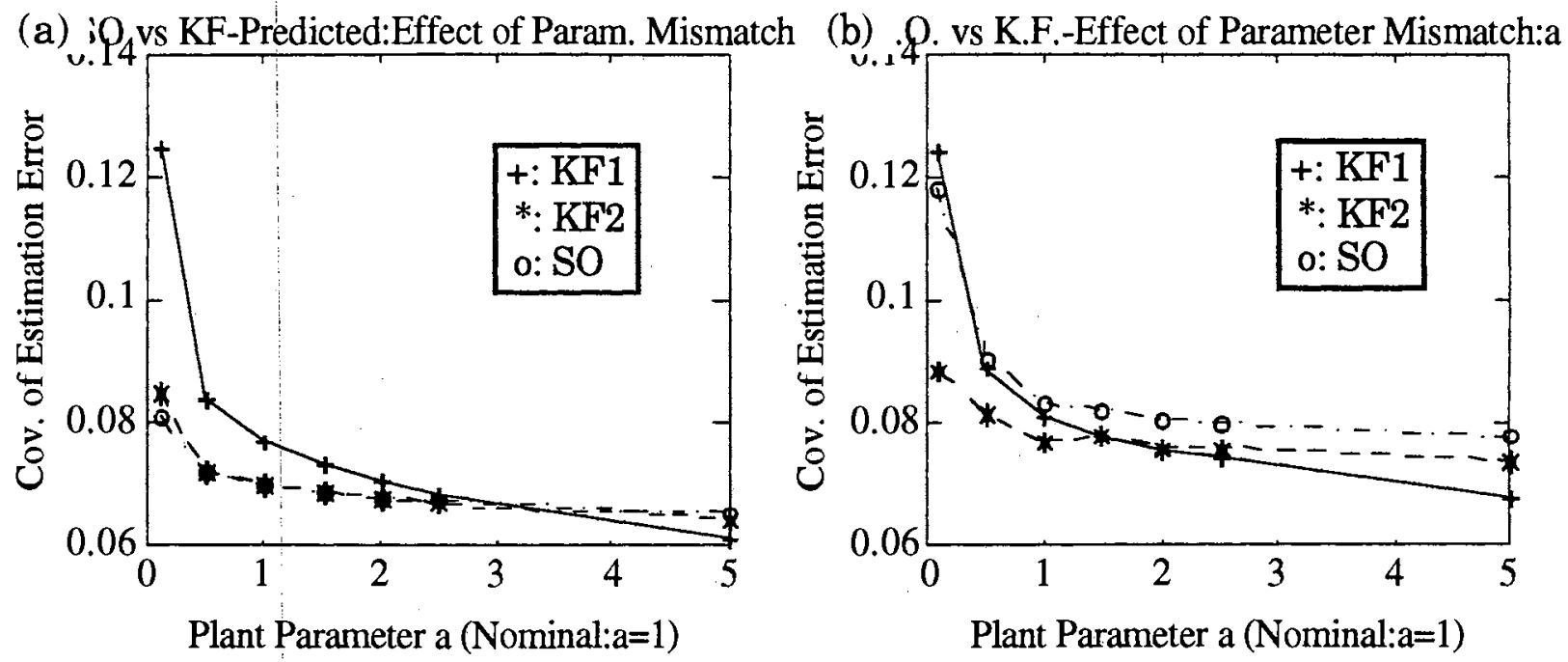

Figure 6.5 Effect of parameter mismatch: $\mathrm{SO}$ vs. KF

(for the 1st order system)

a) Prediction b) Simulated results

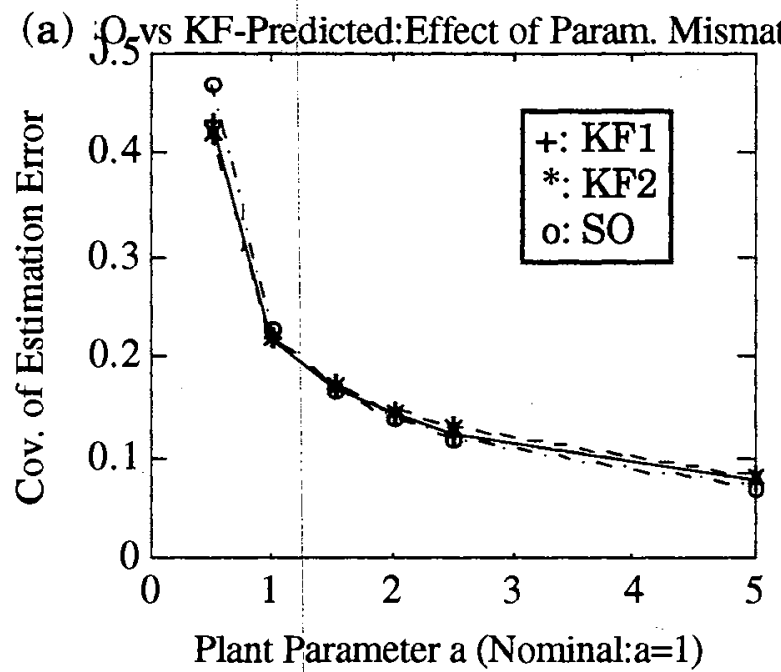

(b) D. vs K.F.-Effect of Parameter Mismatch:a

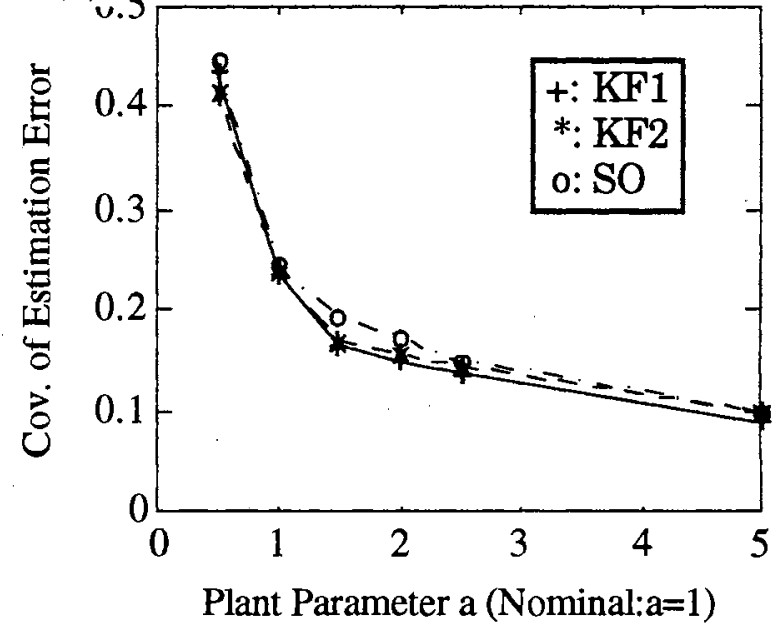

Figure 6.6 Effect of parameter mismatch: SO vs. KF

(for the 2-order system)

a) Prediction b) Simulated results 
The results are shown in Figure 6.5 (a) and Figure 6.6 (a) for prediction and in Figure $6.5(\mathrm{~b})$ and Figure $6.6(\mathrm{~b})$ for simulation. Theoretically, the sliding observer shows good robustness for larger parameter mismatch but the actual simulation is not the best one. For values if actual $\mathrm{A}$ much smaller than the nominal value, the KF2 is the best one and for values if actual A much larger than the nominal value, the KF1 is the best one. This can be explained as the same way as the previous analysis. For small parameter one wants to increase filter gain in order to have a suitable filter, and for large parameter case vice versa. 


\section{CHAPTER VII}

\section{CONCLUSION AND FUTURE RESEARCH}

\subsection{Summary and Conclusion}

\subsubsection{Thesis Summary}

The thesis has approached to the stability of the sliding observer based on the differential geometry of the problem. The coordinate transformation enabled to apply the linear system theorem to the stability Theorem of the sliding observer. A semi-analytic design algorithm was suggested according to the stability theorem. The designed sliding observer was compared with other nonlinear observers for several applications.

In Chapter 1, the robust feature and the solution definition of switching system was introduced. A literature survey on robust and practical nonlinear observers were performed.

In Chapter 2, fundamentals of the sliding observer was investigated and explained systematically on the robust features. The analysis includes the solutions and dynamics of switching system. Emphasis are on the derivation of the coordinate transformation.

In Chapter 3, a algebraic stability condition was derived by the worst case analysis for the 2nd order switching system. It is shown that the worst direction of general order of system can be searched by numerical search method. For practical purposes, an approximate worst direction 
method is suggested. The worst case analysis is generalized for the stability analysis of the sliding observer by introducing the Lyapunov-like function. The general shearing effect is explained by the contour of Lyapunov-like function.

In Chapter 4, a design algorithm "Sliding Observer design by wOrst reaching dynamics for Nonlinear/uncertain system" (SOON) was proposed based on the stability theorems. The sliding observer was designed for 4 practical applications and compared with other current nonlinear observers. The advantages and superior performance of the new design methodology was demonstrated via a simulation. Besides utilizing the linear system theory via the coordinate transformation, the new design algorithm also guarantees stability with known bound of uncertainty and the initial states.

In Chapter 5, the sliding observer for multiple measurement, as a practical example, has been designed and combined with a controller for a two-link robot system. The cases with and without parameter uncertainty were implemented and simulated in the digital computer. In this case the sliding observer and controller were designed independently without examining the separation principle.

In Chapter 6, the performance robustness of the sliding observer, with noises and parameter uncertainty, was analyzed by theoretical prediction and numerical simulation as a extension of Misawa's method. To quasilinearize the error dynamics, a Random Input Describing Function was used as a statistical linearization method. The measurement noise was assumed colored and the process noise was assumed as white gaussian noise. As the results, the sliding observer was designed and analyzed for the 1st and 2nd order Gauss-Markov process. 


\subsubsection{Conclusion}

Most nonlinear observer methods have their own positive aspects, either as extensions of linear techniques or as novel nonlinear techniques, a dual technique of the variable structure control, for instance. A common drawback to the previous nonlinear observer is that the exact nonlinearities of the system must be modeled, either directly or indirectly, into the dynamics of the observer. On the contrary, the sliding observer requires only bounds of uncertainties and nonlinearities of the system in the phase variable canonical form. Furthermore, it guarantees stability with the bounded initial condition, and it can be easily implemented using a microprocessor.

The recent adaptive robust observer of Walcott et al. requires necessarily the matching condition which is difficult to be satisfied practically. On the contrary, the sliding observer can be designed without satisfying the matching condition. The same matching condition was derived by an entirely different way, i.e., the Alimov's transformation (see Appendix B.4 [Alimov, 1960]). One should notice that the design method by passivity theorem (see Appendix B.3 [Misawa, 1988]) also arrived at the same condition. On the contrary, the new stability analysis adopted the linear system theory (i.e., reaching dynamics) so that the conservativeness of the matching condition or strictly negative definite of Lyapunov theorem can be replaced by the strictly decreasing Lyapunov-like function sequence of the passing points. Even though a system has multiple measurements and its states are coupled in the canonical form, the sliding observer can be designed. In this case, the sliding observer error dynamics are decoupled for each measurement.

A new design algorithm "SOON" is proposed according to the stability theorems. Usually by following the design procedure SOON, one can design 
the sliding observer converge directly to the sliding patch. If the observer requirement is not satisfied by the above design procedure SOON, then it can be improved by allowing the solution point pass through the hyperplane in the worst case.

In the numerical examples, it was shown that the new sliding observer effectively adopted to the bounded parameter uncertainty, such as the dry friction and inaccuracies of the system model.

\subsection{Future Research}

\subsubsection{Design Algorithm by Lyapunov-like Stability}

If the observer requirement is not satisfied by the direct converging design procedures (SOON) then it can be improved by allowing the solution point pass through the hyperplane in the worst case. According to Theorem 3.3, if the final Lyapunov-like function $\mathrm{V}_{\mathrm{s}^{*}}^{\mathrm{f}}\left(\sigma\left(\tau_{\mathrm{i}+1}\right)\right)$ is strictly less than the precedent final Lyapunov-like function $\mathrm{V}_{\mathrm{s}}^{\mathrm{f}}\left(\sigma\left(\tau_{\mathrm{i}}\right)\right)$, then the sliding observer is stable. The equation (3.67) is

$$
\mathrm{V}_{\mathrm{s}^{*}}^{\mathrm{f}}\left(\sigma\left(\tau_{\mathrm{i}+1}\right)\right)-\mathrm{V}_{\mathrm{s}}^{\mathrm{f}}\left(\sigma\left(\tau_{\mathrm{i}}\right)\right)<-\left.\rho|| \sigma_{\mathrm{s}}\left(\tau_{\mathrm{j}}\right)\right|^{2}
$$

where the subscripts $\mathrm{s}$ and $\mathrm{s}^{*}$ have different signs: $\operatorname{sign}(\mathrm{s}) \neq \operatorname{sign}\left(\mathrm{s}^{*}\right)$.

If there is no passing at the hyperplane by the direct converge design procedure then we do not need to consider the passing jump. On the contrary, if the solution point passes through the sliding patch then not only should the equation (3.67) be satisfied but also the passing jump of the worst trajectory should be negative in the design procedure based on Theorem 3.3.

In Figure 7.1, even though the passing jump $J_{1}$ was already considered in the equation (3.67), the next passing jump $\mathrm{J}_{2}$ should be negative because 
the positive jump by the worst passing point can make the error dynamics unstable.

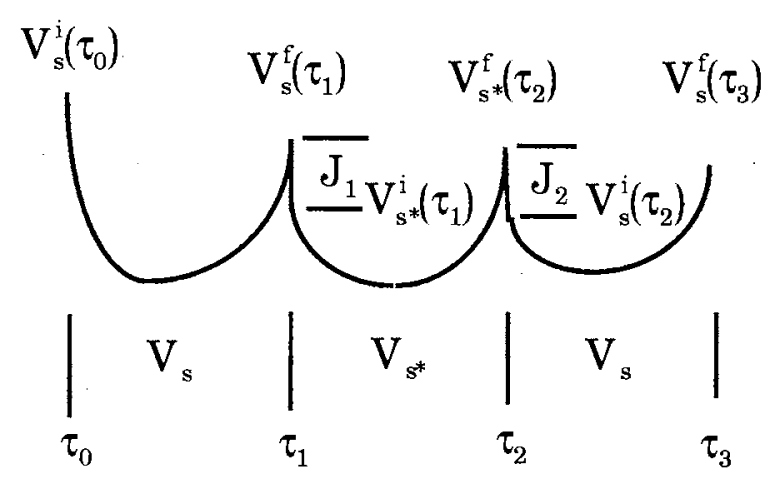

Figure 7.1 Lyapunov-like function and the passing jump

The difference of Lyapunov-like function between the passing points is

$$
\begin{aligned}
J\left(\tau_{j}\right)_{\left(x_{2}>k_{1}\right)} & =V_{+}^{\mathrm{i}}\left(\tau_{j}\right)-V_{-}^{f}\left(\tau_{j}\right) \\
& =-4 K_{s}^{T} \mathrm{P} x \\
& =-4\left[\begin{array}{llll}
0 & k_{1} & \ldots & k_{n-1}
\end{array}\right] P\left[\begin{array}{c}
0 \\
x_{2} \\
\vdots \\
x_{n}
\end{array}\right]
\end{aligned}
$$

In Figure 7.1, even though the passing jump $J_{1}$ was already considered in the equation (3.67), the next passing jump $\mathrm{J}_{2}$ should be negative because the positive jump by the worst passing point can make the error dynamics unstable.

If the sign of the passing state is unity then the passing jump is always negative as shown in Appendix A. However, the sign equalization property is hard to prove analytically as is shown in Appendix C. Hence it is reasonable to search the worst case numerically. 


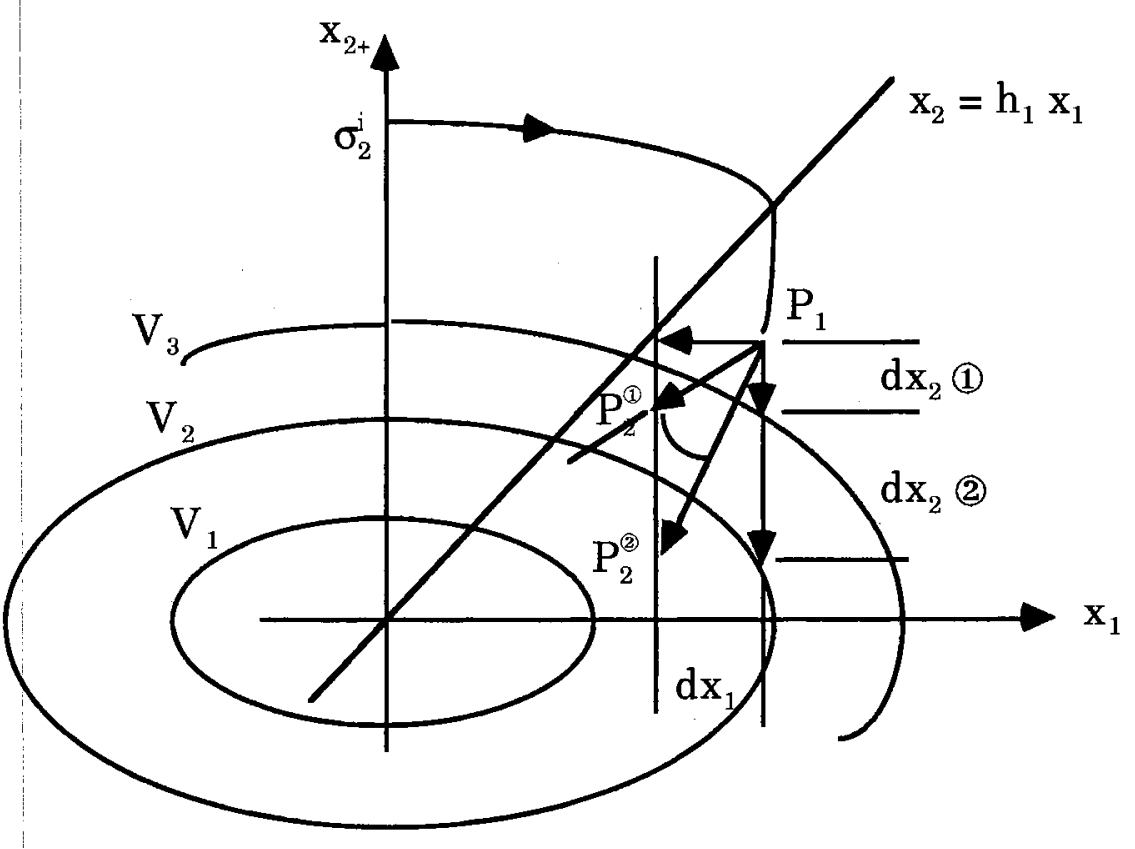

Figure 7.2 Conception of velocity field and Lyapunov-like function

\subsubsection{Worst-case Lyapunov-like Function Search}

Consider the 2nd order reaching dynamics as shown in Figure 7.2. For a finite time $d t$, the difference $d x_{1}, d x_{2}$ of a moving point $P_{1}$ is determined by the position $\mathrm{x}_{1}, \mathrm{x}_{2}$ and the disturbance input $\mathrm{u}_{\mathrm{d}}$. By adding the scaled differences of $d x_{1}$ and $d x_{2}$ to the point $P_{1}$, the fictitious next point $P_{2^{*}}$ was defined by equation (3.22). The numerical search algorithm is the same as the former one except the cost function. In this search, the Lyapunov-like function is the cost function instead of the distance in order to apply the stability Theorem 3.3. Since the switching constant k's determines (see equation 2.54) the accuracy of the observer error, the smaller constant k's increase the accuracy. With these new constants $k$ 's, the passing jump's 
will be computed. In the first design procedure, one might tune the design constants $\mathrm{K}$ and $\mathrm{H}$ already. However, since the available linear correction constant $\mathrm{H}$ is practically limited by noise, the available accuracy by the first design method is also limited. With this proper constant $\mathrm{H}$, one can increase the accuracy by allowing the solution point pass through the hyperplane. The suggested design procedure based on the Lyapunov-like stability Theorem is as follows:

\subsubsection{Design procedure Based on the Lyapunov-like Stability Theorem}

1. Data:

- The maximum bound of the disturbance $w$

- The worst bound of the initial states

- The linear correction constant $\mathrm{H}$

2. Choose the switching constant $\mathrm{K}$ according to the desired observer accuracy.

- For a $\mathrm{n}_{-}$th order case, the suggested coefficient $\mathrm{K}$ is

$$
\frac{\sqrt{\mathrm{P}_{22}}}{\sqrt{\mathrm{P}_{33}}} \approx \frac{\mathrm{k}_{1}}{\mathrm{k}_{2}}, \frac{\sqrt{\mathrm{P}_{33}}}{\sqrt{\mathrm{P}_{44}}} \approx \frac{\mathrm{k}_{2}}{\mathrm{k}_{3}}, \ldots, \frac{\sqrt{\mathrm{P}_{\mathrm{n}-1}}}{\sqrt{\mathrm{P}_{\mathrm{n}}}} \approx \frac{\mathrm{k}_{\mathrm{n}}}{\mathrm{k}_{\mathrm{n}-1}}
$$

- Check sliding dynamics eigenvalues equation (2.24)

- Check steady state error equation (2.54) in the sliding region

3. Simulate SIMNON program Reaching

- Searching the worst Lyapunov-like function

4. Tuning the design constants $\mathrm{K}$

- If equation (4.7) is satisfied and the jump is negative then one can increase accuracy by choosing smaller $\mathrm{K}$ 
- If equation (4.7) is not satisfied or the jump is positive then one should decrease accuracy by choosing larger $\mathrm{K}$

\subsubsection{Design Algorithm for Noisy Measurement}

The performance robustness of the sliding observer, with noises and parameter uncertainty, was analyzed by the theoretical prediction and the numerical simulation in Chapter 6. In order to quasi-linearize the error dynamics, a Random Input Describing Function was used as a statistical linearization method. However, the noise characteristics may change according to the variation of the operating condition. In this case, it is necessary to adjust the filter according to the varying noise characteristics: the solution of this problem is usually sought in a class of adaptive systems in which the noise intensities are estimated in one way or another. However, such adaptive systems may be complicated and require more computational power. Furthermore, in a situation in which over long time intervals, the system do not need any adjustment, and the adaptive part of the filter will be idle.

For the practical purpose, it is interesting to design a filter which would obtain a suboptimal estimation error, but it is insensitive to inexact noise intensity.

\subsubsection{Using Nonlinear Terms in the Observer}

As shown in Chapter 4 and Chapter 5, the nonlinear model sliding observer improves the accuracy of estimation. Therefore, as far as the computational power is allowed, it is preferred to include the proper nonlinear terms in the observer structure. But, if the state estimation error is large, 
for example, the initial condition is mismatched, the nonlinear term possibly induces a negative effect [Misawa, 1988].

It is difficult to set up a general rule in using the nonlinear terms. Hence, for the practical purpose, it can be studied as case studies, the estimators for the robot systems in the chapter 5 , for instance.

A comparative study between nonlinear models and simple linear models is needed:

- Convergence speed and computational load

- Develop a computational index to compare computational burdens of different nonlinear observers.

This study should be useful as a design criteria. 


\section{BIBLIOGRAPHY}

Alimov, Y. I., "Lyapunov Functions for Relay Control Systems", Automation and Remote Control,Vol. 21, No. 6, pp. 500-506, Dec. 1960.

Alimov, Y. I., "On the Application of Lyapunov's Direct Method to Differential Equations with Ambiguous Right Sides", Automation and Remote Control,Vol. 22, No. 7, pp. 713-725, Dec. 1961.

Ambrosino, G., Celentano, G. and Garofalo, F., "Variable Structure Model Reference Adaptive Control Systems", Int. J. of Control,Vol. 39, No. 6, pp. 1339-1349, 1984.

Anderson, B. D. O., Bitmead, R. R., Johnson, C. R. J., Kokotovic, P. V. and Kosut, R. L., Stability of Adaptive Systems: Passivity and Averaging Analysis, MIT Press, Cambridge, Mass, 1986.

Anulova, S. V., "Random Disturbances of the Operation of Control Systems in the Sliding Mode", Automation and Remote Control,Vol. pp. April 1986.

Arie, T., "An Adaptive Steering System for a Ship", IEEE Control System Magazine,Vol. 6, pp. 3-8, Oct. 1986.

Asada, H. and Slotine, J. J. E., Robust Analysis and Control, John Wiley and Sons, 1986.

Astrom, K. J., Introduction to Stochastic Control Theory, Academic Press, 1970.

Athans, M., Kapasouris, P., Kappos, E. and III, H. A. S., "Linear-Quadratic Gaussian with Loop-Transfer Recovery Methodology for the F-100 Engine", J. Guidance,Vol. 9, No. 1, pp. 45-52, Jan.-Feb. 1986.

Banks, S. P., "A note on Non-linear Observers", Int. J. Control,Vol. 34 , No. 1, pp. 185-190, 1981.

Baumann, W. T. and Rugh, W. J., "Feedback Control of Nonlinear Systems by Extended Linearization", IEEE Trans. Automatic Control,Vol. AC31, No. 1, pp. Jan. 1986.

Beaman, J. J., "Non-linear Quadratic Gausian Control", Int. J. of Control,Vol. 39, No. 2, pp. 343-361, 1984. 
Bellgardt, K., Kuhlmann, W. and Meyer, H., "Application of an Extended Kalman Filter for State Estimation of a Yeast Fermentation", Control Theory and Application,Vol. 133, pp. 226-34, Sept. 1986.

Bestle, D. and Zeitz, M., "Canonical form Observer Design for Nonlinear Time-variable Systems", Int. J. Control,Vol. 38, No. 2, pp. 419-431, 1983.

Bockman, S. F., "Lyapunov Exponents for Systems Described by Differential Equations with Discontinuous Right-Hand Sides", ACC, Boston, MA,pp. 1673-1678, 1991.

Brockett, R. W. and Byrnes, C. I., "Multivariable Nyquist Criteria, Root Loci, And Pole Placement: A Geometric Viewpoint", IEEE Trans. on Autom. Contr.,Vol. AC-26, No. 1, pp. 271-284, Feb. 1981.

Brogliato, B., Landau, I.-D. and Lozan0-Leal, R., "Adaptive Motion Control of Robot Manipulators: A Unified Approach Based on Passivity", ACC, San Diego, CA,pp. 2259-2264, 1990.

Chang, L.-W., "A versatile Sliding Control with a Second-Order Sliding Condition", $A C C$, Boston,MA,pp. 54-55, 1991.

Chen, Y. H., "On the Robustness of Mismatched Uncertain Dynamical Systems", J. of Dynamic Systems, Measurement and Control,Vol. 109, pp. 29-35, March 1987.

Chen, Y. H., "Large-Scale Uncertain System Under Insufficient Decentralized Controllers", J. of Dynamic Systems, Measurement and Control,Vol. 111, pp. 359-63, Sept. 1989.

Chen, Y. H., "Adaptive Robust Observers for Nonlinear Uncertain Systems", Int. J. of Systems Science,Vol. 21, No. 5, pp. 803-814, May 1990a.

Chen, Y. H., "State Estimation for Nonlinear Uncertain Systems: A Design based on Properties Related to the Uncertainty Bound", Int. J. Control,Vol. 52, No. 5, pp. 1131-1146, 1990 b.

Chen, Y. H. and Hsu, C., "Structural Decomposition Approach for the Stability of Uncertain Dynamic Systems", J. of Applied Mechanics, Vol. 55, pp. 992-4, Dec. 1988.

Chen, Y. H. and Tomizuka, M., "Design of Adaptive Observer for Plant Under Input Disturbance and Measurement Noise", Int. J. of Control,Vol. 47, pp. 625-632, 1988.

Chiang, R. Y. and Safonov, M. G., "Design of $\mathrm{H}_{\infty}$ Controller for a Lightly Damped System using a Bilinear Pole Shifting Trnsform", ACC, 
Boston, MA,pp. 1927-1928, 1991.

Choi, B.-O. and Krishnamurthy , K., "Position and Force Control of Flexible Robotic Manipulators Using the LQG/LTR Design Methodology", ACC, Boston, MA,pp. 1913-1914, 1991.

Craig, J. J., Hsu, P. and Sastry, S., "Adaptive Control of Mechanical Manipulators", IEEE Int. Conf. Robotics Automat., San Francisco, CA,pp. 1986.

Cristi, R., Healey, A. J. and Papoulias, F., "Dynamic Output Feedback by Robust Observer and Variable Structure Control", ACC, San Diego, CA,pp. 2649-2653, 1990.

DeCarlo, R. A., Zak, S. H. and Mathews, G. P., "Variable Structure Control of Nonlinear Multi Variable Systems: A Tutorial", Proceeding of the IEEE, 212-231, 1988.

Desoer, C. A. and Vidyasagar, M., Feedback Systems: Input-output Properties, Academic Press, NewYork, NY, 1975.

Doyle, J. C., "Analysis of Feedback Systems with Structured Uncertianties", IEE Proc.,Vol. 129, Pt. D, No. 6, pp. 242-250, Nov. 1982.

Doyle, J. C., "Structured Uncertainty in Control System Design", Proc. of 24th Conference on Decision and Control, Ft. Lauderdale, FL,pp. 1985.

Doyle, J. C. and Stein, G., "Robustness with Observer", IEEE Trans. on Autom. Contr.,Vol. AC-24, no. 4, pp. 607-611, Aug. 1979.

Esfandiari, F. and Khalil, H. K., "Observer-based Control of Fully-Linearized Nonlinear Systems", the 28th IEEE Conference on Decision and Control, Tampa, FL,pp. 84-89, 1989.

Filippov, A. F., "Differential Equations with Discontinuous Right Hand Sides", Am. Math. Soc. Trans.,Vol. 42, pp. 199-231, 1964.

Filippov, A. F., Differential equations with Discontinuous Righthand Sides, Kluwer Academic Publishers, 1988.

Friedland, B., Control System Design, McGraw-Hill, 1986.

Frimm, F., Evaluation of Ship's Steering Characteristics - Full Scale and Model Test, Master Thesis, Escola Politecnica da Univeridade de Sao Paulo, 1983

Gao, Z. and Antsaklis, P. J., "New Bounds on Parameter Uncertainties for Robust Stability", ACC, Boston, MA,pp. 879-880, 1991. 
Gelb, A., Applied Optimal Estimation, MIT. Press, Cambridge, M.A., 1974.

Gelb, A. and Vandervelde, W. E., Multiple-Input Describing Functions and Non-linear System Design, McGraw-Hill Book Co., New York, 1968.

Gharban, C. K. and Cory, B. J., "Nonlinear Dynamic Power System State Estimation", IEEE Transactions on Power Systems,Vol. 1, pp. 276-83, Aug. 1986.

Gibson, J. E., Nonlinear Automatic Control, McGraw-Hill Inc., 1963.

Glad, S. T., "On the Gain Margin of Nonlinear and Optimal Regulators", Proc. of 21st Conf. on Decision \& Control, Orland, FL,pp. 957-962, 1982.

Gopalswamy, S. and Hedrick, J. K., "Robust Adaptive Control of Multivariable Nonlinear Systems", ACC, San Diego, CA,pp. 2247-2252, 1990.

Grossman, W., Khorrami, F. and Friedland, B., "An Observer-Based Design for Robust Control of Robot Manipulators", $A C C$, San Diego, CA,pp. 731-736, 1990.

Grunberg, D. B., A Methodology for Design Robust Multivariable Nonlinear Control Systems, MIT, 1986

Habibi, S. R. and Richards, R. J., "Sliding Mode Control of an Electrically Powered Industrial Robot", IEE Proc.-D,Vol. 139, No. 2, pp. 207-226, March 1992.

Hached, M., Esfahani, S. M. M. and Zak, S. H., "On the Stability and Estimation of Ultimate Boundedness of Nonlinear/Uncertain Dynamic Systems with Bounded Controllers", ACC, San Diego, CA,pp. 1180-1185, 1990.

Haessig, D. A., Jr., "On the Modeling and Simulation of Friction", ACC, San Diego, CA,pp. 1256-1261, 1990.

Hagedorn, P., Non-Linear Oscillations, Oxford University Press, Oxford, 1988.

Hermann, R. and Krener, A. J., "Nonlinear Controllability and Observability", IEEE Trans. on Autom. Contr.,Vol. AC-22, No. 5, pp. 728-740, Oct. 1977.

Holmes, P., "Dynamics of a Nonlinear Oscillator with Feedback Control I : Local Analysis", J. of Dynamic Systems, Measurement, and Control,Vol. 107, pp. 159-165, June 1985.

Holten, L., Gjelsvik, A. and Asm, S., "Comparison of Different Methods for 
State Estimation", IEEE Trans. on Power Systems,Vol. 3, pp. 1798-804, Nov. 1988.

Hori, N., Nikiforuk, P. N. and Kanai, K., "On the Improvement of on Adaptive Observer for Multi-output Systems", IEE Procedings. Part D., Control Theory and Applications,Vol. 135, pp. 67-71, Jan. 1988.

Hsia, T. C., "Adaptive Control of Robot Manipulators - A Review", IEEE Int. Conf. on Robotics and Automation, 1986.

$\mathrm{Hu}, \mathrm{X}$. , "A Note on Nonlinear State Observers", ACC, Boston, MA,pp. 717-718, 1991.

Hunt, L. R., Su, R. and Meyer, G., "Global Transformation of Nonlinear Systems", IEEE Trans. on Autom. Contr.,Vol. AC-28, No. 1, pp. 24-30, Jan. 1983.

Hunt, L. R. and Verma, M. S., "Observers and Controllers for Feedback Linearizable systems", ACC, Boston, MA,pp. 546-547, 1991.

Itkis, V., Control Systems of Variable Structure, John-Wiley, 1976.

Jang, S. S., B., J. and Mukai, H., "Comparison of Two Approaches to On-line Parameter and State Estimation of Nonlinear Systems", Ind. \& Eng. Chemistry Proc. Design and Development,Vol. 25, pp. 809-14, July 1986.

Jazwinski, A. H., Stochastic Process and Filtering Theory, Academic Press, New York, 1970.

Kailath, T., Linear Systems, Prentice-Hall, Inc., Englewood Cliffs, NJ, 1980.

Kalman, R. E., "A New Approach to Linear Filtering and Prediction Problems", Trans. ASME (J. Basic Engineering),Vol. 82D, no. 1, pp. 35-45, March 1960.

Kalman, R. E. and Bucy, R. S., "New Results in Linear Filtering and Prediction Theory", Trans. ASME (J. of Basic Engineering),Vol. 83D, No. 1, pp. 95-108, March 1961.

Kanellakopoulos, I. and Kokotovic, P. V., "Observer-based Adaptive Control of Nonlinear Systems Under Matching Conditions", ACC, San Diego, CA,pp. 549-555, 1991.

Kantor, J. C., "Finite Demensional Nonlinear Observer for an Exothermic Stirred-Tank Reactor", Chemical Eng. Science,Vol. 44, No. 7, pp. 1503$1510,1989$.

Kao, C. K. and Sinha, A., "Sliding Mode Control of Vibration in Flexible 
Structures Using Estimated States", ACC, Boston, MA,pp. 2467-2474, 1991.

Kaplan, W., Ordinary Differential Equations, Addition-wesley, Reading, Massachusetts, 1958.

Keller, H., "Nonlinear Observer Design by Transformation into a Generalized Observer Canonical Form", Int. J. Control,Vol. 46, No. 6, pp. 1915-1930, 1987.

Kolmogorov, A. N. and Fomin, S. V., Introductory Real Analysis, Dover Publications, Inc., New York, NY, 1975.

Kolodziej, E. and Mohler, R. R., "State Estimation and Control of Conditional Linear Systems", SIAM J. on Cont. and Optimization,Vol. 24, pp. 497-508, May 1986.

Krener, A. J. and Isidori, A., "Linearization by Output Injection and Nonlinear Observer", Systems \& Control Letters,Vol. 3, pp. 47-52, June 1983.

Krener, A. J. and Respondek, W., "Nonlinear Observers with Linearizable Error dynamics", SIAM J. of Control and Optimization,Vol. 23, No. 2, pp. 197-216, Mar. 1985.

Landau, Y. D., Adaptive Control, The Model Reference Approach, Marcel Decker Inc., New York, 1979.

Li, C. W. and Tao, L. W., "Observing Nonlinear Time-variable Systems Through a Canonical Form Observer", Int. J. Control,Vol. 44, No.6, pp. 1703-1713, 1986.

Li, Y. and Lee, E. B., "A Synthesis Appoach to Uncertain Systems with Parametric Uncertainties", ACC, Boston, MA,pp. 2718-2719, 1991.

Ljung, L., System Identification, Prentice-Hall, Inc., 1987.

Ljung, L. and Soderstrom, T., Theory and Practice of Recursive Identification, MIT Press, Cambridge, Mass., 1983.

Luenberger, D. G., "An Introduction to Observer", IEEE Trans. on Autom. Contr.,Vol. AC-16, No. 6, pp. 596-602, Dec. 1971.

Macfarlane, A. G. J. and Postlethwaite, I., "The Generalized nyquist Stability Criterion and Multivariable Root Loci", Int. J. of Control,Vol. 25, No. 1, pp. 81-127, 1977.

Masmoudi, R. A. and Hedrick, J. K., "Estimation of Vehicle Shaft Torque Using Nonlinear Observers", ASME Winter Annual Meeting, 1987. 
Mendel, J. M., Lessons in Digital Estimation Theory, Prentice-Hall, Inc., 1987.

Minamide, N., Nikiforuk, P. N. and Gupta, M. M., "Design of a ReducedOrder Adaptive Observer", IEE Procedings. Part D. Control Theory and Applications,Vol. 133, pp. 133-6, May 1986.

Misawa, E. A., Nonlinear State Estimation Using Sliding Observers, Massachusetts Institute of Technology, Ph.D. Thesis, 1988

Misawa, E. A. and Hedrick, J. K., "Nonlinear Observers-A State-of-the-Art Survey", Trans. of the ASME,Vol. III, pp. 344, Sept. 1989.

Misawa, E. A., Hedrick, J. K., Slotine, J.-J. E. and Verghese, G. C., "Sliding Observer Design for Nonlinear State Estimation", IFAC Symposium on Nonlinear Control Systems Design, Capri, Italy,pp. 1989.

Moher, R. R, and Bugnon, J. F., "Nonlinear System Perspective and Guaranteed State Estimation", IEEE Int. Sympo. on Circuits and Systems 1989, the 22nd ISCAS., 1989.

Moose, R. L., Sistanizadeh, M. K. and Skagfjord, G., "Adaptive State Estimation for a System with Unknown Input and Measurement Bias", IEEE J: of Ocean Eng.,Vol. 12, pp. 222-7, Jan. 1987.

Nandam, P. K. and Sen, P. C., "A Comparative Study of a Luenberger Observer and Adaptive Observer-based Variable Structure Speed Control System Using a Self-Controlled Synchronous Motor", IEEE Transactons on Ind ustrial Electronics,Vol. 37, pp. 127-132, Apr. 1990.

Narendra, K. S. and Taylor, J. H., Frequency Domain Criteria for Absolute Stability, Academic Press, New York, 1973.

Nayfeh, A. H. and Mook, D. T., Nonlinear Oscillations, John Wiley \& Sons, New York, 1979.

Nicosia, S. and Tomei, P., "State Observation of Elastic Joint Robots", Robot Control 1988(SYROCO 1988)-Selected papers from the 2nd IFAC Symposium, 1988.

Nicosia, S., Tomei, P. and Tornambe, A., "A Nonlinear Observer for Elastic Robots", Unpublished,Vol. pp. 1986.

Nicosia, S., Tomei, P. and Tornambe, A., "Nonlinear Control and Observation Algorithms for a Single-link Flexible Robot Arm", Int. J. of Control,Vol. 49, pp. 829-840, Mar. 1989.

Nicosia, S., Tomei, P. and Tornambe, A., "Observer-based Control Law for 
a Class of Nonlinear Systems", Int. J. of Control,Vol. 51, pp. 553-566, Mar 1990.

Nicosia, S. Ni and Tornambe, A., "High-gain Observers in the State and Parameter Estimation of Robots Having Elastic Joints", Systems and Control Letters,Vol. 13, pp. 331-337, Nov. 1989.

Niemann, H. H. N., Stroustrup, J. and Sogaard-Andersen, P., "General Conditions for Loop Transfer Recovery", $A C C$, Boston, MA,pp. 333-334, 1991.

Ortega, R. and Spong, M. W., "Adaptive Motion Control of Rigid Robots: A Tutorial", IEEE CDC, Austin, TX,pp. 1988.

Ortega, R. and Yu, T., "Robustness of Direct Adaptive Controllers: A Servey", 10th IFAC World Conf., Munich, FRG,pp. 1986.

Peleties, P. and Decarlo, R., "Asymptotic Stability of m-Switched Systems using Lyapunov-Like Functions", ACC, Boston, MA,pp. 1679-1684, 1991.

Phaneuf, R. J, Approximate Nonlinear Estimation, Massachusetts Institute of Technology, 1968

Phelps, A. R., "Computation of Nonlinear Observers", the 28th IEEE Conference on Decision and Control, Tempa, FL,pp. 1989.

Pons, M. N. and Engasser, J. M., "Comparison Between Adaptive and ModelBased Extended Kalman Filters", ACC, 1989.

Raghavan, S. and Hedrick, J. K., "Slidng Compensators for a Class of Nonlinear Systems", ACC, San Diego, CA,pp. 1174-1179, 1990.

Schweppe, F. C., Uncertain Dynamic Systems, Prentice-Hall, Inc., Englewood Cliffs, NJ, 1973.

Sethna, P. R., "Method of averaging for systems bounded for positive time", J. Math. Anal. Appl.,Vol. 41, pp. 621-631, 1973.

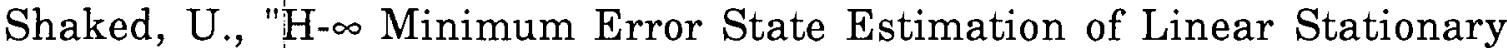
Processes", IEEE Trans. on Auto. Contr.,Vol. 35 No.5, pp. 554-558, May 1990.

Shamma, J. S., "The Necessity of the Small-Gain Theorem for Time-Varying and Nonlinear Systems", IEEE Trans. on Auto. Contr.,Vol. 36, No. 10, pp. 1138-1147, Oct 1991.

Shamma, J. S. and Dahleh, M. A., "Time-Varying Versus Time-Invariant Compensation for Rejection of Presistent Bounded Disturbances and Robust Stabilization", IEEE Trans. Autom. Contr.,Vol. 36, No. 7, pp. 
838-847, July 1991.

Sims, C. S. and Wilson, D., "EXtended Optimality Properties of ReducedOrder Observers", ACC, Boston, MA,pp. 2823-2824, 1991.

Slotine, J. and Coetsee, J. A., "Adaptive Sliding Controller Synthesis for Nonlinear Systems", Int. J. Control,Vol. 43, pp. 1631-1651, 1986.

Slotine, J.-J., Hedrick, J. K. and Misawa, E. A., "On Sliding Observers for Nonlinear Systems", J. of Dynamic Systems, Measurement, and Control,Vol. 109, pp. 245, Sept. 1987.

Slotine, J.-J. and Li, W., Applied Nonlinear Control, Prentice-Hall, 1991.

Slotine, J.-J. E., "Sliding Controller Design for Nonlinear Systems", Int. J. Control,Vol. 40, No. 2, pp. 421-434, 1984.

Sobol, I. M., The Monte Carlo Method, Mir Publishers, Moscow, 1987.

Sorenson, H. W., Kalman Filtering: Theory and Application, IEEE Press, New York, NY, 1985.

Spong, M. W. and Ortega, R., "On Adaptive Inverse Dynamics Control of Rigid Robots", IEEE trans. on Automatic Control,Vol. 35 No. 1, pp. 92-95, Jan. 1990.

Spong, M. W. and Vidyasagar, M., "Robust Linear Compensator Design for Nonlinear Robotic Control", IEEE J. Robotics Automat.,Vol. RA-3, pp. 345-351, 1987.

Spong, M. W. and Vidyasagar, M., Robot Dynamics and Control, Wiley, New York, NY, 1989.

Stalford, H., "Stability Conditions for Nonlinear Control Processes Using Lyapunov Functions with Discontinuous Derivatives", $J$. of Mathematical Analysis and Applications,Vol. 84, pp. 356-371, 1981.

Steinberg, A. and Corless, M., "Output Feedback Stabilization of Uncertain Dynamical Systems", IEEE Trans. on Autom. Cont.,Vol. 30, No. 10, pp. 1025-1027, Oct 1985.

Stephenson, Al, "On a new type of dynamical stability", Mem. Proc. Manch. Lit. Phil. Soc.,Vol. 52, pp. 1907-1908, 1908.

Svobodny, T. P., "Stability of Nonlinear Observers for Dissipative ODEs", Int. J. of Control,Vol. 50, No. 4, pp. 1235-1247, Oct. 1989.

Swiniarski, R., "Adaptive Observer Appilication to the Estimation of Nonlinear Biotechnological Processes", Adaptive Control of Chemical 
Processes 1988(ADCHEM 1988) - Selected Papers from the 2nd Int. IFAC Symposium, 1988.

Taylor, J. H., "Statistical Performance Analysis of Nonlinear Stochastic Systems by the Monte Carlo Method", Mathematics and Computers in Simulation,Vol. XXIII, pp. 21-33, 1981.

Thau, F. E., "Observing the State of Nonlinear Dynamical Systems", Int. J. Control,Vol. 17, No.3, pp. 471-479, 1973.

Tibken, B. and Hofer, E. P., "Systematic Observer Design for Bilinear Systems", IEEE International symposium on Circuits and Systems, the 22nd ISCAS, 1989.

Tkachenko, V. Y., "Simulation of Dry Friction", Automation and Remote Control,Vol. 35, pp. March 1974.

Tornambo, A., "Use of Asymtotic Observers Having-high-gain in the State and Parameter Estimation", the 28th IEEE Conference on Decision and Control, 1989.

Tunali, E. T. and Tarn, T.-J., "New Results for Identifiablity of Nonlinear Systems", IEEE Trans. on Autom. Contr.,Vol. AC-32, No. 2, pp. 146-154, Feb. 1987.

Uosaki, K. and Koketsu, Y., "Optimal Robust Transformation for Nonlinear State Estimation", Eighth IFAC/IFORS Symposium on Identification and System Parameter Estimation, 1988.

Utkin, V. I., "Survey Paper: Variable Structure Systems with Sliding Modes", IEEE Trans. on Autom. Cont.,Vol. AC-22, No. 2, pp. 212-222, April 1977.

Utkin, V. I., Sliding Modes and Their Application in Variable Structure System, MIR Publishers, Moskow, 1978.

Utkin, V. I., "Variable Structure Systems: Present and Future", Automation and Remote Control,Vol. 44, No. 9, pp. 1105-1120, Sept. 1984.

Utkin, V. I. and Drakunov, S. V., "Stochastic Regularization of Systems with Discontinuous Controls", Sov. Phys. Dokl.,Vol. 28(10), pp. 835-837, Oct. 1983.

Velichenko, V. V., "Optimal Control Problems for Equations with Discontinuous Right-Hand Sides", Automation and Remote Control,Vol. 27, No.7, pp. 1153-1163, July 1966.

Walcott, B. and Zak, S. H., "Combined Observer-Controller Synthesis for Uncertain Dynamical Systems with Applications", IEEE Trans. on 
System, Man, and Cyber.,Vol. 18 No. 1, pp. 88-104, 1988.

Walcott, B. L., "Nonlinear Output Stabization of Uncertain Systems", ACC, San Diego, CA,pp. 2253-2258, 1990.

Walcott, B. L., Corless, M. J. and S.H. Zak, "Comparative Study of Nonlinear State-Observation Techniques", Int. J. Control,Vol. 45, No. 6, pp. 21092132, 1987.

Walcott, B. L. and Zak, S. H., "Observation and Control of Nonlinear Uncertain Dynamical Systems: A Variable Structure Approach", Purdue Univ., 1986a.

Walcott, B. L. and Zak, S. H., "Observation of Dynamical Systems in the Presence of Bounded Nonlinearities/Uncertainties", 25th CDC, Athens; Greece,pp. 1986b.

Walcott, B. L. and Zak, S. H., "State Observation of Nonlinear Uncertain Dynamical Systems", IEEE Trans. Autom. Cont.,Vol. AC-32, pp. 166$170,1987$.

Wishner, R. P., Tabaczynski, J. A. and M. Athans, "A Comparison of Three Non-Linear Filters", Automatica, Pergamon Press,Vol. 5, pp. 487-496, 1969.

Wit, C. C. D. and Alvarez, Q., "Nonlinear Observer for Robots with Flexible Joints", ?, 1988.

Wit, C. C. D. and Slotine, J.-J. E., "Sliding Observer for Robot Manipulators", Automatica,Vol. 27, No. 5, pp. 859-864, 1991.

Xia, X.-H. and Gao, W.-B., "Nonlinear Observer Design by Observer Error Linearization", SIAM J. Control and Optimization,Vol. 27, N0. 1, pp. 199-216, Jan. 1989.

Yaz, E. and Azemi, A., "Variable Structure Observers for Nonlinear Models with Unbounded Noise and Measurement Uncertainties", $A C C$, Boston, MA,pp. 43-48, 1991.

Zak, S. H., Brehove, J. D. and Corless, M. J., "Control of Uncertain Systems with Unmodeled Actuator and Sensor Dynamics and Incomplete State Information", IEEE Trans. on Systems, Man and Cybernetics, Vol. 19, No. 2, pp. 241-257, Mar-Apr. 1989.

Zak, S. H. and MacCarley, C. A., "State-Feedback Control of Non-linear Systems", Int. J. Control,Vol. 43, No. 5, pp. 1497-1514, 1986.

Zeitz, M., "The Extended Luenberger Observer for Nonlinear Systems", Systems \& Control Letters,Vol. 9, pp. 149-156, 1987. 
Zhao, X., Stalford, H. L. and Hanagud, S. V., "On the mu Control of Uncertain Manipulator Arms", ACC, Boston, MA,pp. 2051-2056, 1991.

Zhu, W.-h., Chen, H.-t. and Zhang, Z.-j., "A Variable Structure Robot Control Algorithm with an Observer", IEEE Trans. on Robotics and Automation, Vol. 8 No. 4, pp. 486-492, Aug. 1992. 


\section{APPENDIX A}

\section{MATHEMATICAL BACKGROUND}

\section{A.1 Differential Equationswith Discontinuous}

Right-Hand Sides

The evolution of the theory of differential equations with discontinuous right-hand sides has been to a great extent motivated by its many applications. The popular usage of the switching technique in automatic control systems leads to the necessity of fabricating an intricate theory. The technique of the variable structure system with sliding motions has been developed fundamentally in the literature from Russia [Utkin, 1984]. The basic properties are explored in this chapter.

\section{Definitions of solution}

The solutions of the differential equations with discontinuous right-hand sides are studied by cases. The usual definition of a solution for a continuous differential equation cannot apply directly to the discontinuous differential equations of which are discontinuous on an arbitrary smooth line or on a surface S.

i) For the first case, the definition of a solution for continuous differential equations can be applied to the case in which the solution point approach on the surface $\mathrm{S}$ and leave on the other side of the surface $\mathrm{S}$. Here the solution passes through $\mathrm{S}$ and satisfies the equation everywhere except at the 
intersection point at which the solution does not have a derivative.

ii) In the other case, a solution point approaches on both sides of a line or a hyperplane $\mathrm{S}$, the usual definition is unsuitable because there is no clue of how a solution point that has reached on the hyperplane S may be continued. The solution of velocity field along a surface of discontinuous, i.e. the hyperplane (in this study $\mathrm{x}_{1}=0$ ), can be determined by the Filippov' equivalent solution.

Consider the case in which the function $f(x)$ is discontinuous on a smooth surface $\mathrm{S}$ that is given by the equation $\mathrm{y}=\mathrm{Cx}=0$. The surface $\mathrm{S}$ separates its neighborhood in the state space into domains $\Omega_{\text {. }}$ and $\Omega_{+}$. As the trajectory $\xi$ approaches the point $\mathrm{x} \in \mathrm{S}$ from the domains $\Omega$. and $\Omega_{+}$at a given time t. let the function $f(t, \xi)$ have the limiting values:

$$
\lim _{\substack{\xi \xi \mathbb{S}^{-} \\ \xi \rightarrow S(x)}} f(t, \xi)=f(t, x), \lim _{\substack{\xi \in \mathbb{S}^{+} \\ \xi \rightarrow S(x)}} f(t, \xi)=f^{+}(t, x)
$$

Lemma 1.1.[Filippov, 1964] Let the regions $\Omega_{+}$and $\Omega_{-}$in the space $\mathrm{x}_{1}, \ldots$ ., $\mathrm{x}_{\mathrm{n}}$ be separated by a smooth surface $\mathrm{S}$. Suppose that the vector function $\mathrm{f}(\mathrm{t}, \mathrm{x})$ is bounded and, for any time $t$, its limiting values $\mathrm{f}^{\mathrm{f}}(\mathrm{t}, \mathrm{x})$ and $\mathrm{f}^{\mathbf{4}}(t, \mathrm{x})$ exist when the solution point is approaches from $\mathrm{S}_{-}$and $\mathrm{S}_{+}$. Let $\mathrm{f}_{\mathrm{N}}$ and $\mathrm{f}_{\mathrm{N}}^{+}$be the projections of the vectors $\mathrm{f}$ and $\mathrm{f}^{+}$on the normal to the surface $\mathrm{S}$ directed from $S_{\text {. and }} S_{+}$. Let the vector function $x(t)$ be absolutely continuous. For $t_{1} \leq t \leq t_{2}$, let assume $x(t) \in S, f_{N}(t, x) \geq 0, f_{N}^{+}(t, x) \leq 0, f_{N}(t, x)-f_{N}^{+}(t, x)>0$. In order for $x(t)$ to be a solution of (A.1), it is necessary and sufficient that for almost all $t \in\left[t_{1}, t_{2}\right]$

$$
\dot{\mathrm{x}}=\mathrm{f}^{0}(\mathrm{t}, \mathrm{x}), \mathrm{f}^{0} \equiv \alpha \mathrm{f}^{+}+(1-\alpha) \mathrm{f}^{\mathrm{f}}
$$

where, $\alpha \equiv \frac{f_{N}}{f_{N}^{-}-f_{N}^{*}}$ 
Proof) See Filippov pp.206.

$\underline{\text { Remark })}$ If $f$ and $f^{+}$are continuous with respect to $(t, x), x \in S$, then equation (A.2) holds for all $t \in\left(t_{1}, t_{2}\right)$. It is easy to see that the value of $\dot{x}$ for a solution is lying on the hyperplane.

Following Figure A.1 are examples of hyperplane $H=x_{1}=0$.
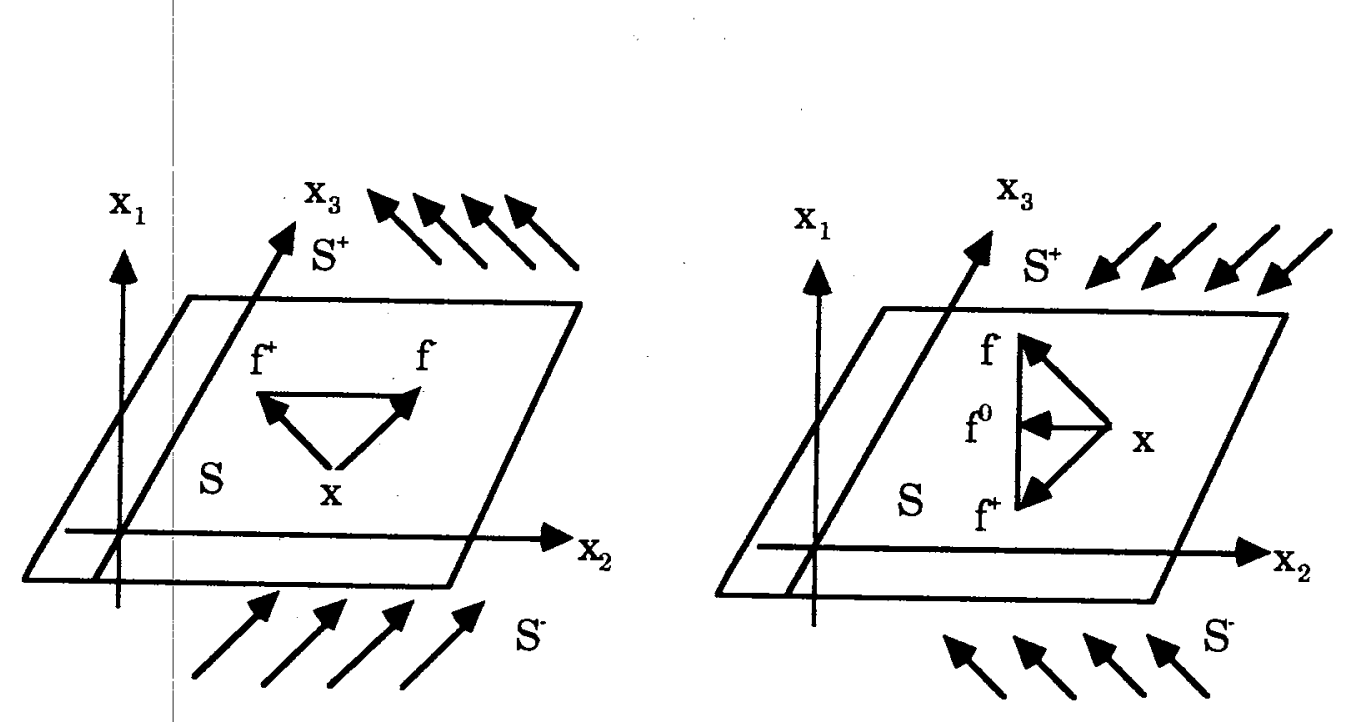

Figure A.1 The velocity field near the hyperplane

For $t_{1}<t<t_{2}$, draw a line joining the endpoints of the vectors $f(t, x)$ and $\mathrm{f}^{+}(t, x)$ that start from the point $x$. If this line does not intersect the hyperplane $\mathrm{S}$ as shown Figure A.1a, the solution is determined by the first case. In this case, the solutions pass from one side to the other of the hyperplane.

If this line intersects the hyperplane S (Figure A.1b), the intersection point is the endpoint of the vector $f^{0}(t, x)$ which determines the velocity of motion $\dot{x}=f^{f}(t, x)$ along the surface $S$. This is the Filippov's equivalent solution [Filippov, 1964] of the differential equation with discontinuous righthand sides: 


$$
\dot{x}=f^{0}(t, x)
$$

The velocity function of this case is:

$$
f^{o}(t, x) \neq f(t, x), \quad f^{o}(t, x) \neq f^{+}(t, x)
$$

This movement of the solution point is called a "sliding motion".

\section{A.2 Differential of Signum Function}

We want to apply chain rule to differentiate a signum function about time which is discontinuous at zero. The Fourier series expansion for a square wave is obtained as followings:

$$
\operatorname{sqw}(x)=\frac{4}{\pi} \sin \left(\frac{\pi x}{p}\right)+\frac{4}{3 \pi} \sin \left(\frac{3 \pi x}{p}\right)+\ldots+\frac{4}{(2 m-1) \pi} \sin \left(\frac{(2 m-1) \pi x}{p}\right)+\ldots
$$
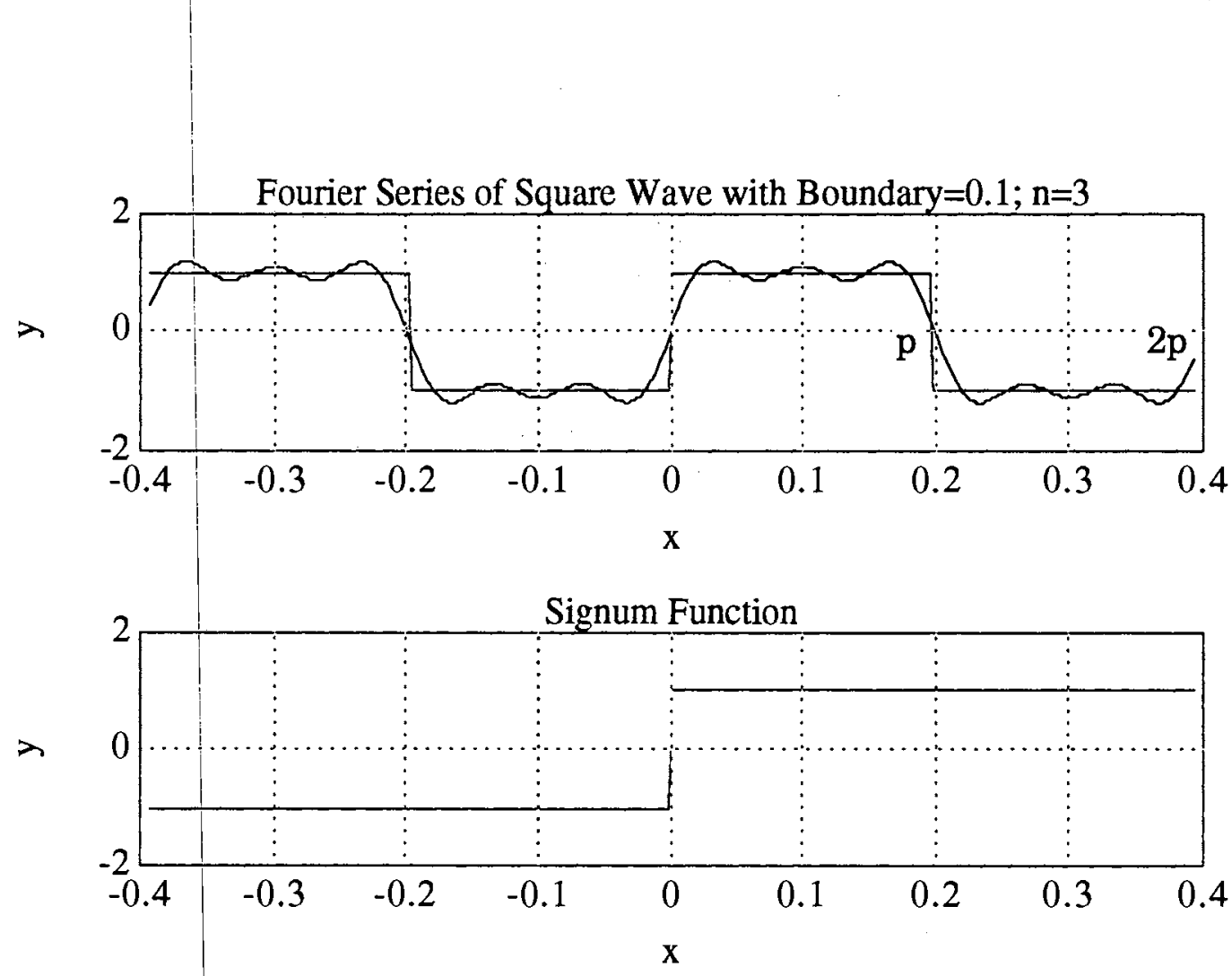

Figure A.2 Fourier series expansion of square wave and signum function 
The signum function can be recomposed by using $\operatorname{sqw}(\mathbf{x})$ and $\operatorname{sgn}(\mathrm{x})$.

$$
\operatorname{sgn}(x)= \begin{cases}\operatorname{sqw}(x) & (-\varepsilon<x<\varepsilon) \\ \operatorname{sgn}(x) & (x<-\varepsilon>, x>\varepsilon)\end{cases}
$$

$$
\begin{aligned}
& \frac{d}{d t}(\operatorname{sgn}(x))= \\
= & \frac{d}{d t}\left[\frac{4}{\pi}\left\{\sin \left(\frac{\pi x}{p}\right)+\frac{1}{3} \sin \left(\frac{3 \pi x}{p}\right)+\ldots+\frac{1}{(2 m-1)} \sin \left(\frac{(2 m-1) \pi x}{p}\right)+\ldots\right\}\right] \\
= & \frac{4}{\pi}\left[\frac{d}{d t}\left\{\sin \left(\frac{\pi x}{p}\right)\right\}+\frac{1}{3} \frac{d}{d t}\left\{\sin \left(\frac{3 \pi x}{p}\right)\right\}+\ldots+\frac{1}{(2 m-1)} \frac{d}{d t}\left\{\sin \left(\frac{(2 m-1) \pi x}{p}\right)+\ldots\right\}\right] \\
= & \frac{4}{\pi}\left[\frac{d}{d x}\left\{\sin \left(\frac{\pi x}{p}\right)\right)\left(\frac{d x}{d t}+\frac{1}{3} \frac{d}{d x}\left\{\sin \left(\frac{3 \pi x}{p}\right)\right\} \frac{d x}{d t}+\ldots+\frac{1}{(2 m-1)} \frac{d}{d x}\left\{\sin \left(\frac{(2 m-1) \pi x}{p}\right) \frac{d x}{d t}+\ldots\right\}\right)\right] \\
= & \frac{4}{\pi}\left[\frac{\pi}{p}\left\{\cos \left(\frac{\pi x}{p}\right)\right\} \frac{d x}{d t}+\frac{1}{3} \frac{3 \pi}{p}\left\{\cos \left(\frac{3 \pi x}{p}\right)\left|\frac{d x}{d t}+\ldots+\frac{1}{(2 m-1)} \frac{(2 m-1) \pi}{p}\right| \cos \left(\frac{(2 m-1) \pi x}{p}\right) \frac{d x}{d t}\right\}+\ldots\right. \\
= & \frac{4}{\pi} \frac{\pi}{p}\left[\left\{\cos \left(\frac{\pi x}{p}\right)\right\}\left\{\frac{d x}{d t}+\left\{\cos \left(\frac{3 \pi x}{p}\right)\right\} \frac{d x}{d t}+\ldots+\left\{\cos \left(\frac{(2 m-1) \pi x}{p}\right) \frac{d x}{d t}\right\}+\ldots\right]\right. \\
= & \frac{4}{p}\left[\left\{\cos \left(\frac{\pi x}{p}\right)\right\} \frac{d x}{d t}+\left\{\cos \left(\frac{3 \pi x}{p}\right)\right\} \frac{d x}{d t}+\ldots+\left\{\cos \left(\frac{(2 n-1) \pi x}{p}\right) \frac{d x}{d t}\right\}+\ldots\right] \\
= & \frac{4}{p}\left[\cos \left(\frac{\pi x}{p}\right)+\cos \left(\frac{3 \pi x}{p}\right)+\ldots+\cos \left(\frac{(2 m-1) \pi x}{p}\right)+\ldots\right] \frac{d x}{d t}
\end{aligned}
$$

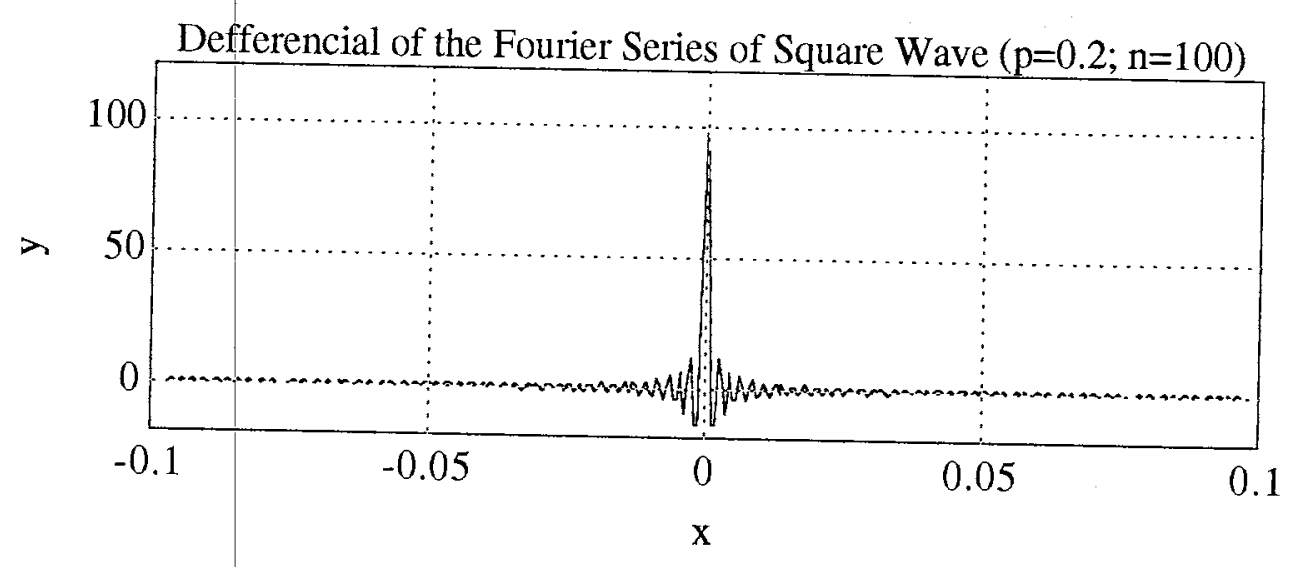

Figure A.3 Differential of the Fourier Series of Square Wave 
For finite $p, 4 / p$ is also finite. Hence, we need to evaluate the rest term.

$$
\text { Let } y=\left[\cos \left(\frac{\pi x}{p}\right)+\cos \left(\frac{3 \pi x}{p}\right)+\ldots+\cos \left(\frac{(2 m-1) \pi x}{p}\right)+\ldots\right]
$$

If $\mathrm{x}$ equals zero, then $\mathrm{y}$ will be infinite as $\mathrm{n}$ goes infinite. If $\mathrm{x}$ does not equal zero, then $\mathrm{y}$ will converge zero as $\mathrm{n}$ goes infinite. We can define $\delta(\mathrm{x})$ as following:

$$
\delta(x)=\left\{\begin{array}{l}
\infty,(x=0) \\
0,(x \neq 0)
\end{array}\right.
$$

Let us consider a following function:

$$
\begin{aligned}
& f_{p}(x)= \begin{cases}\frac{1}{\varepsilon}(-\varepsilon<x<\varepsilon) \\
-\frac{1}{\varepsilon}(-p<x<-p+\varepsilon, p-\varepsilon<x<p) \\
0 \text { elsewhere }\end{cases} \\
& f(x)=\frac{a_{o}}{2}+\sum_{n=1}^{\infty}\left(a_{n} \cos \left(\frac{n \pi x}{p}\right)+b_{n} \sin \left(\frac{n \pi x}{p}\right)\right) \\
& \text { where } a_{o}=0, a_{n}=\frac{2}{p} \int_{0}^{\varepsilon} \frac{1}{\varepsilon} \cos \left(\frac{n \pi x}{p}\right) d t+\frac{2}{p} \int_{p \cdot \varepsilon}^{p} \frac{-1}{\varepsilon} \cos \left(\frac{n \pi x}{p}\right) d t \\
& a_{2 m}=0
\end{aligned}
$$

i) $\mathrm{n}=2 \mathrm{~m}$

ii) $\mathrm{n}=2 \mathrm{~m}-1$

$$
a_{2 \mathrm{~m}-1}=\frac{4}{\mathrm{p}}\left[\frac{\sin \left(\frac{(2 \mathrm{~m}-1) \pi \varepsilon}{\mathrm{p}}\right)}{\frac{(2 \mathrm{~m}-1) \pi \varepsilon}{\mathrm{p}}}\right]
$$

It is well known that a delta function is mathematically defined as a limit of the above function. Take the limit and we get the coefficient:

$$
\lim _{\varepsilon \rightarrow \infty} a_{2 m-1}=\frac{4}{p}
$$

We can see this coefficient is exactly same as the former one. 


\section{A.3 Differential of Saturation Function}

The differential of saturation function is:

$$
\frac{d}{d t}\left(\operatorname{sat}\left(x_{1}\right)\right)=\left\{\begin{array}{c}
\left.\frac{1}{\varepsilon} \dot{x}_{1},\left(-\varepsilon<x_{1}<\varepsilon\right)\right) \\
0,\left(\left|x_{1}\right|>\varepsilon\right)
\end{array}\right.
$$

Definition A.1

$$
\delta_{\varepsilon}(x)=\left\{\begin{array}{l}
\frac{1}{\varepsilon}, \text { for }-\varepsilon<x<\varepsilon \\
0, \text { for }|x|>\varepsilon
\end{array}\right.
$$

By using definition A.1, the derivative of $\operatorname{sat}\left(\mathrm{x}_{1}\right)$ is:

$$
\frac{d}{d t}\left(\operatorname{sat}\left(x_{1}\right)\right)=\delta_{\varepsilon}\left(x_{1}\right) \dot{x}_{1}
$$

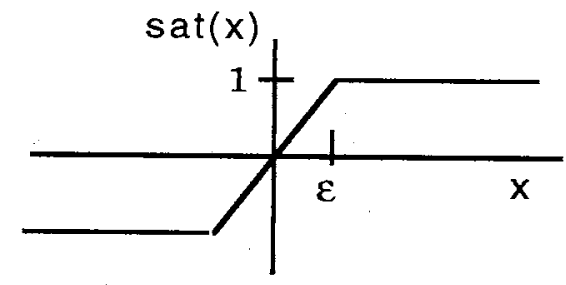

Figure A.4 A saturation function

\section{Definition A.2}

\section{A.4 Bilinear Form Matrix Function}

A nxn matrix $P$ whose diagonal elements $\left|p_{i j}\right|>\sum_{j \neq i}^{n}\left|p_{i j}\right|, \forall i$, is said diagonally dominant (row dominance) [Strang, 1976].

A nxn matrix $P$ whose diagonal elements $\left|p_{i j}\right|>\sum_{j \neq i}^{n}\left|p_{j}\right|, \forall i$, is said diagonally dominant (column dominance). 
For any symmetric matrix $\mathrm{P}$, the product $\mathrm{Q}(\mathrm{x})=\mathrm{x}^{\mathrm{t}} \mathrm{Px}$ is a quadratic form.

Theorem A.1

A necessary and sufficient condition that the real quadratic form $Q(x)=x^{t}$ $\mathrm{Px}$ be positive-definite is that every principal minor of $\mathrm{P}$ be positive.

Closely associated with quadratic form is what is known as bilinear form.

\section{Definition A.3}

If $\mathrm{P}$ is a symmetric matrix, the expression $\mathrm{B}(\mathrm{x}, \mathrm{y})=\mathrm{x}^{\mathrm{t}} \mathrm{Py}$ is said as a bilinear form.

Consider a simple conjecture that every element of $\mathrm{x}, \mathrm{y}$ and every principal minor of $\mathrm{P}$ of a bilinear form $\mathrm{B}(\mathrm{x}, \mathrm{y})$ is positive. However, following example shows this conjecture is not true.

$$
\mathrm{B}(\mathrm{x}, \mathrm{y})=\mathrm{x}^{\mathrm{T}} \mathrm{Py}=\left[\begin{array}{ll}
1 & 5
\end{array}\right]\left[\begin{array}{rr}
9 & -5 \\
-5 & 3
\end{array}\right]\left[\begin{array}{l}
1 \\
1
\end{array}\right]=-6
$$

Let us consider a special form $J=K^{\mathrm{T}} \mathrm{P} x=\mathrm{D} x$ where $\mathrm{D}=\mathrm{K}^{\mathrm{T}} \mathrm{P}$. If every element $\mathrm{D}$ and $\mathrm{x}$ is positive then $\mathrm{J}$ is positive. For the sliding observer, matrix $P$ is obtained form Lyapunov equation with the Hurwitz matrix $A_{m}$ and the positive coefficient $\mathrm{K}$ is another design coefficient. For designing a sliding observer we can easily check the matrix D. However, a diagonal dominant matrix $\mathrm{P}$ and positive coefficient $\mathrm{K}$ will make a positive matrix $\mathrm{D}$. 


\section{APPENDIX B}

\section{STABILITY THEOREMS}

\section{B.1 Linear Stability Theorem}

\section{$\underline{\text { Time-invariant case }}$}

Although various stability conditions have been obtained for linear, timevarying dynamical equations, they can hardly be used, because all the conditions are stated in terms of state transition matrices, which are very difficult, if not impossible, to obtain. In the stability study of linear timeinvariant dynamical equations, the knowledge of the state transition matrix is, however, not needed. The stability can be determined directly form the system matrix A. Consider the n-dimensional linear time-invariant dynamical equation

$$
\begin{aligned}
\dot{x} & =A x+B u \\
y & =C x
\end{aligned}
$$

where $\mathrm{A}, \mathrm{B}, \mathrm{C}$ are $\mathrm{nxn}, \mathrm{nxp}$, qxn real constant matrices, respectively. As in the time-varying case, first, we study the zero-state response and the zeroinput response and then the total response. The zero-state response of the system is characterized by

$$
\mathrm{G}(\mathrm{s})=\mathrm{C}(\mathrm{s} \mathrm{I}-\mathrm{A})^{-1} \mathrm{~B}
$$

From theorem, the forced response of system is BIBO stable if and only if all the poles of $\mathrm{G}(\mathrm{s})$ have negative real parts. 
A linear dynamical equation is said to be totally stable if and only if for any initial state and for any bounded input, the output as will as all the state variables are bounded [Chen, 1970].

\section{Theorem B.1}

The forced response (zero state) of $\dot{x}=A x$ is asymptotically stable if and only if all the eigenvalues of A have negative real parts.

Proof) Let $\mathrm{P}$ be the nonsingular matrix such that $\widehat{\mathrm{A}}=\mathrm{PAP}^{-1}$ and $\widehat{\mathrm{A}}$ is in the Jordan form. For the zero state response, in order to be asymtotically stable, in addition to the bound of $\left\|\mathrm{e}^{\mathrm{A} t}\right\|$, it is required that $\left\|\mathrm{e}^{\mathrm{A} t}\right\|$ tends to zero as $t \rightarrow \infty$. or equivalently, that $\left\|\mathrm{e}^{\hat{\mathrm{A}}}\right\| \rightarrow 0$ as $t \rightarrow \infty$. Since every entry of $\mathrm{e}^{\hat{\mathrm{A} t}}$ is of the form $t^{\mathrm{k}} \mathrm{e}^{\mathrm{a}_{j} \mathrm{t}^{\mathrm{i}} \mathrm{w}_{\mathrm{j}} \mathrm{t}}$, we conclude that $\left\|\mathrm{e}^{\hat{A} t}\right\| \rightarrow 0$ as $\mathrm{t} \rightarrow \infty$ if and only if all the eigenvalues of $\widehat{A}$. Consequently the eigenvalues of $\mathrm{A}$ have negative real parts.

If a linear time-invariant system is asymtotically stable, its zero-input response will approach zero exponentially; thus it is also said to be exponentially stable. It is clear that total stability implies BIBO stability. However, BIBO stability may not imply total stability. If a linear timeinvariant dynamical equation is controllable and observable, then the characteristic polynomial of $\mathrm{A}$ is equal to the characteristic polynomial of G(s). Consequently, we have the following theorem [Chen, 1970].

If a linear time-invariant dynamical equation is controllable and observable, then the following statements are equivalent:

1. The dynamical equation is totally stable.

2. The forced response is BIBO stable.

3. The forced response is asymtotically stable. 
4. All the poles of the transfer function matrix have negative real parts.

5. All the eigenvalues of the matrix $A$ have negative real parts.

\section{B.2 Dynamics of Saturation Nonlinear Observer}

Rewrite the suggested sliding observer (2.6) with the saturation functions instead of the signum functions. Consider a 3-dimensional system:

$$
\left\{\begin{array}{l}
\dot{\mathrm{x}}_{1}=\mathrm{x}_{2}-\mathrm{h}_{1} \mathrm{x}_{1}-\mathrm{k}_{1} \operatorname{sat}\left(\mathrm{x}_{1}\right) \\
\dot{\mathrm{x}}_{2}=\mathrm{x}_{3}-\mathrm{h}_{2} \mathrm{x}_{1}-\mathrm{k}_{2} \operatorname{sat}\left(\mathrm{x}_{1}\right) \\
\dot{\mathrm{x}}_{3}=-\mathrm{h}_{3} \mathrm{x}_{1}-\mathrm{k}_{3} \operatorname{sat}\left(\mathrm{x}_{1}\right)+\mathrm{w}
\end{array}\right.
$$

If the switching term $1_{\mathrm{s}}$ is a saturation function, the equivalent gain is

$$
\begin{aligned}
& \dot{\hat{x}}=A \hat{x}+B u+H \widetilde{y}+K 1_{s}(\widetilde{y}) \\
& \text { where } \quad 1_{\mathrm{s}}(\tilde{\mathrm{y}})= \begin{cases}\frac{\tilde{\mathrm{y}}}{\varepsilon}, & |\tilde{\mathrm{y}}| \leq \varepsilon \\
\frac{\tilde{\mathrm{y}}}{|\widetilde{y}|}, & |\widetilde{\mathrm{y}}|>\varepsilon\end{cases} \\
& |\tilde{y}| \leq \varepsilon, \dot{\hat{x}}=A \hat{x}+B u+\left(H+\frac{K}{\varepsilon}\right) \tilde{y} \\
& |\widetilde{y}|>\varepsilon, \quad \dot{\hat{x}}=A \hat{x}+B u+\left(H+\frac{K}{|\widetilde{y}|}\right) \tilde{y}
\end{aligned}
$$

The robust property of the saturation function needs to be studied to design a quasi-optimal filter. The equivalent gain $h^{*}$ is plotted as:

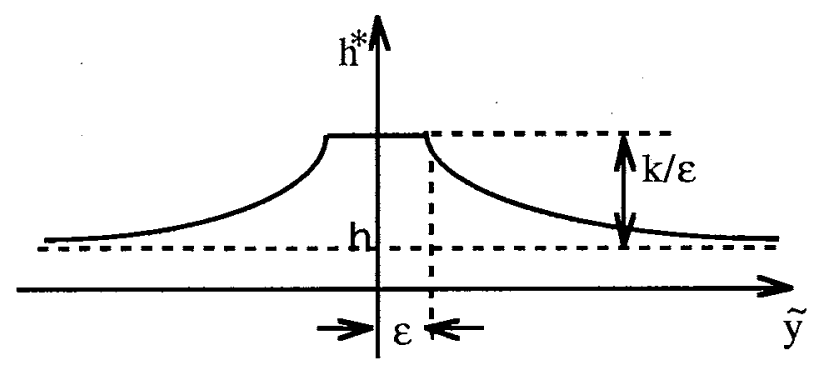

Figure B.1 The equivalent gain profile 
The derivative of sat $\left(x_{1}\right)$ is expressed by using definition A.17:

$$
\frac{\mathrm{d}}{\mathrm{dt}}\left(\operatorname{sat}\left(\mathrm{x}_{1}\right)\right)=\delta_{\varepsilon}\left(\mathrm{x}_{1}\right) \dot{\mathbf{x}}_{1}
$$

Differentiate the first equation and plug in the second equation:

$$
\ddot{x}_{1}=-h_{1} \dot{x}_{1}-h_{2} x_{1}-k_{1} \delta_{\varepsilon}\left(x_{1}\right) \dot{x}_{1}-k_{2} \operatorname{sat}\left(x_{1}\right)+x_{3}
$$

Differentiate again and plug in the third equation:

$$
\mathrm{x}_{1}^{(3)}=-\mathrm{h}_{1} \ddot{\mathrm{x}}_{1}-\mathrm{h}_{2} \dot{\mathrm{x}}_{1}-\mathrm{h}_{3} \mathrm{x}_{1}-\mathrm{k}_{1} \delta_{\varepsilon}\left(\mathrm{x}_{1}\right) \ddot{\mathrm{x}}_{1}-\mathrm{k}_{2} \delta_{\varepsilon}\left(\mathrm{x}_{1}\right) \dot{\mathrm{x}}_{1}-\mathrm{k}_{3} \operatorname{sat}\left(\mathrm{x}_{1}\right)+\mathrm{w}
$$

Combine the same order terms and rewrite the equation then we have the dynamics of the sliding observer with saturation functions:

$$
\mathbf{x}_{1}^{(3)}=-\left(h_{1}+k_{1} \delta_{\varepsilon}\left(x_{1}\right)\right) \ddot{x}_{1}-\left(h_{2}+k_{2} \delta_{\varepsilon}\left(x_{1}\right)\right) \dot{x}_{1}-h_{3} x_{1}-k_{3} \operatorname{sat}\left(x_{1}\right)+w
$$

For convenience, we can rewrite (B.10) for the outside and for the inside of the boundary layer. For the outside of the boundary layer:

$$
x_{1}^{(3)}=-h_{1} \ddot{x}_{1}-h_{2} \dot{x}_{1}-h_{3} x_{1}-k_{3} \operatorname{sgn}\left(x_{1}\right)+w
$$

Within the boundary layer:

$$
\mathbf{x}_{1}^{(3)}=-\left(h_{1}+\frac{k_{1}}{\varepsilon}\right) \ddot{x}_{1}-\left(h_{2}+\frac{k_{2}}{\varepsilon}\right) \dot{x}_{1}-\left(h_{3}+\frac{k_{3}}{\varepsilon}\right) x_{1}+w
$$

Outside the boundary layer, the sliding observer with saturation functions behaves exactly the same as the signum function observer. Within the boundary layer, the sliding observer with saturation functions behaves like a high gain system. 
B.3 Sliding Observer using the Absolute Stability Theorem

In this section, the sliding observer by Misawa [1988] is summarized. Let us consider the robust nonlinear observer in the presence of only output measurement, rather than full state feedback. Since, the measured output provides only partial information about the system state, the additional observer structure must be used. Misawa [1988] proved the stability of the sliding observer by using the Passivity theorem [Astrom, 1989] that guarantees the $\mathrm{L}_{2}$-stability of the estimation error. In order to maintain generality, we need to consider the following conditions:

- Define the terms $1_{s}$ such that $\widetilde{y}^{\mathrm{T}} 1_{s}>0$, and $k_{n}^{*}>0$

With these conditions, one can combine the disturbance and "switching" term $1_{s}(\tilde{y})$, resulting in the estimation error dynamics described by:

$$
\dot{\widetilde{x}}=(A-H ~ C) \widetilde{x}-K^{*} \alpha(\tilde{y}) \tilde{y}
$$

This equation is described in the block diagram as Figure B.2. One can readily see that the equation (B.5) is now given in the exact form required by the Passivity theorem.

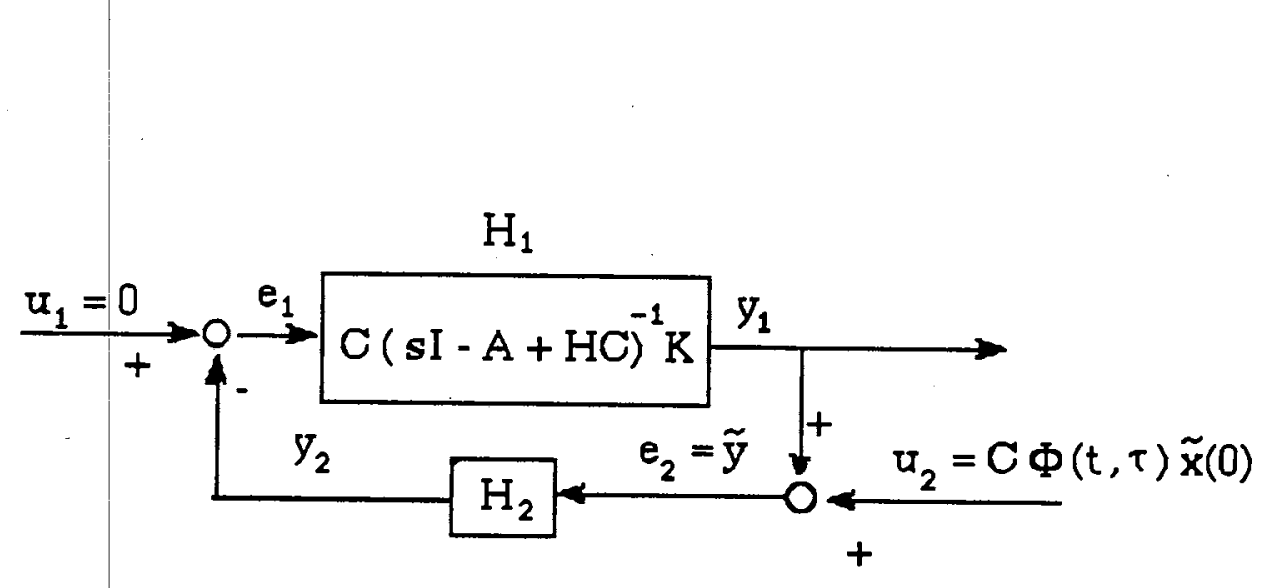

Figure B.2 Estimation error dynamics 
The operators $\mathrm{H}_{1}$ and $\mathrm{H}_{2}$, and the input $\mathrm{u}_{2}$ are defined as follows:

$$
\begin{aligned}
& \mathrm{H}_{1} \mathrm{e}_{1}:=\mathrm{C} \int_{0}^{t} \Phi(t, \tau) \mathrm{K}(\tau) \mathrm{e}_{1}(\tau) d \tau \\
& \mathrm{H}_{2} \mathrm{e}_{2}=\mathrm{H}_{2} \tilde{\mathrm{y}}:=\alpha(\widetilde{\mathrm{y}}) \tilde{\mathrm{y}} \\
& \mathrm{u}_{2}:=\mathrm{C} \Phi(t, \tau) \widetilde{\mathrm{x}}(0)
\end{aligned}
$$

where $\Phi(t)=e^{(A-H C) t}$ is the state transition matrix.

In this scheme, $e_{1}$ and $e_{2}$ are defined as: $e_{1}=u_{1}-H_{2} e_{2}$ and $e_{2}=u_{2}+H_{1} e_{1}$.

In order to introduce the main result, it is necessary to define the sets $\mathcal{H}$ and $\mathrm{He}$ :

$$
\begin{aligned}
& \mathscr{H}=\left\{\mathrm{x}(\mathrm{t}) \mid\|\mathrm{x}\|^{2}=\int_{0}^{\infty} \mathrm{x}(\mathrm{t})^{\mathrm{T}} \mathrm{x}(\mathrm{t}) \mathrm{dt}<\infty\right\} \\
& \mathscr{H}_{e}=\left\{\mathrm{x}(\mathrm{t}) \mid\|\mathrm{x}\|_{\mathrm{T}}^{2}=\int_{0}^{\mathrm{T}} \mathrm{x}(\mathrm{t})^{\mathrm{T}} \mathrm{x}(\mathrm{t}) \mathrm{dt}<\infty\right\} \quad \forall \mathrm{T} \geq 0
\end{aligned}
$$

The main estimation convergence (stability) results can now be stated as a theorem.

\section{Theorem B.2}

- ( A - H C ) is made stable;

- The gain matrices $\mathrm{H}$ and $\mathrm{K}$, and the function $1_{\mathrm{s}}$ are chosen such that there are constants $\alpha_{i}$ and $\beta_{i},(i=1,2,3)$, so that:

$$
\begin{aligned}
& \left\|\mathrm{H}_{1} \mathrm{z}\right\|_{T} \leq \alpha_{1}\|z\|_{T}+\beta_{1} \\
& \int_{0}^{T} z(t)^{T}\left(H_{1} z\right) d t=:\left\langle z \mid H_{1} z\right\rangle_{T} \geq \alpha_{2}\|z\|_{T}^{2}+\beta_{2} \\
& \int_{0}^{T}\left(H_{2} z\right)^{T} z(t) d t=:\left\langle H_{2} z \mid z\right\rangle_{T} \geq \alpha_{3}\left\|H_{2} z\right\|_{T}^{2}+\beta_{3}
\end{aligned}
$$


$\forall \mathrm{z} \in \mathcal{H}_{e}, \forall \mathrm{T} \in[0, \infty)$

- $\alpha_{2}+\alpha_{3}>0$;

Then: $\mathrm{e}_{1}, \mathrm{e}_{2}=\widetilde{\mathrm{y}}, \mathrm{H}_{1} \mathrm{e}_{1}, \mathrm{H}_{2} \mathrm{e}_{2} \in \mathcal{H}$, and $\widetilde{\mathrm{y}}=\mathrm{C} \widetilde{\mathrm{x}} \Rightarrow 0$ as $\mathrm{t} \Rightarrow \infty$.

The main result stated in this section gives a fairly general result for the problem at hand.

\section{First Design Procedure}

The first design procedure shows the observed states to be asymptotically convergent if the transfer function matrix

$$
\mathrm{H}_{1}(\mathrm{~s})=\mathrm{C}(\mathrm{sI}-\mathrm{A}+\mathrm{H} \mathrm{C})^{-1} \mathrm{D}
$$

can be made strictly positive real.

1) Let the function $1 \mathrm{~s}($.$) be$

$$
1_{s}(\widetilde{\mathbf{y}})^{T}=\left[\operatorname{sign}\left(\widetilde{\mathbf{y}}_{1}\right), \operatorname{sign}\left(\widetilde{\mathbf{y}}_{2}\right), \ldots, \operatorname{sign}\left(\tilde{\mathrm{y}}_{\mathrm{m}}\right)\right]
$$

where $\operatorname{sign}\left(\widetilde{\mathrm{y}}_{\mathrm{i}}\right)=\tilde{\mathrm{y}} /\left|\tilde{\mathrm{y}}_{\mathrm{i}}\right|$

2) Choose the gain matrices $H$ and $\rho$ so that the following conditions are satisfied:

$$
\begin{aligned}
& P(A-H C)+(A-H C)^{T} P=-Q \\
& K^{T} P=C \\
& K=D \rho
\end{aligned}
$$

for some symmetric positive definite matrices $\mathrm{P}$ and $\mathrm{Q}$.

3) If these conditions are met, then the design is complete. If they are not met one can iterate by changing the matrices $D$ and $\rho$, or iterate using the multiplier theory [Desoer, 1975] trying to meet the conditions given in this design procedure. If these changes do not work, then one can try the 
method described in Second Design Procedure.

The second conditions is usually hard to verify and can be too restrictive for particular problems. When it works, this design procedure guarantees that $\widetilde{\mathrm{y}} \Rightarrow 0$ and $\widehat{\mathrm{x}} \Rightarrow 0$ as $\mathrm{t} \Rightarrow \infty$.

For systems with a single measurement, the definition of strictly positive real linear systems [Desoer, 1975] give an easier way to check whether the operator $\mathrm{H}_{1}$ is strictly positive real. In this case, by placing the eigenvalues of $\mathrm{A}-\mathrm{H} \mathrm{C}$ in the open left half plane, the operator $\mathrm{H}_{1}$ is strictly positive real if and only if the following transfer function

$$
\mathrm{H}_{1}(\mathrm{~s})=\mathrm{C}(\mathrm{sI}-\mathrm{A}+\mathrm{HC})^{-1} \mathrm{~K}
$$

evaluate at all $\mathrm{s}=\mathrm{jw}$ is completely contained in the open right half plane:

$$
\operatorname{Re}[G(j \omega)]>0 \text { for all } \omega \in R
$$

If this approach does not work, one still has the option to design the observer using a following alternative method.

\section{Second Design Procedure}

The previous design procedure was restricted by the Positive Real Lemma, which resulted from the strict passivity condition imposed by the use of pure switching (signum function) for the function $1_{s}(\tilde{y})$. This suggests that by using the saturation function for $1_{s}(\widetilde{y})$, a less restrictive condition may be found. This is the case, and the design procedure uses the circle criterion [Landau, 1979] for the single measurement case, and the extension of circle criterion to the multivariable case [Luenberger, 1966].

In this case the design process has two parts. The first part guarantees that the output estimation error $\widetilde{y}=y-C \widetilde{x}$ remains inside the boundary layer once it gets into it and the second part guarantees that the boundary 
layer is attractive.

1) Let $1_{s}(\widetilde{y})$ be the vector function whose components are saturation functions defined in the usual way:

$$
1_{\mathrm{s}}(\widetilde{\mathbf{y}})^{T}=\left[\operatorname{sat}\left(\widetilde{\mathbf{y}}_{1} / \varepsilon_{1}\right), \operatorname{sat}\left(\widetilde{\mathbf{y}}_{2} / \varepsilon_{2}\right), \ldots, \operatorname{sat}\left(\widetilde{\mathbf{y}}_{\mathrm{m}} / \varepsilon_{\mathrm{m}}\right)\right]
$$

and

$$
\operatorname{sat}\left(\widetilde{\mathbf{y}}_{\mathrm{i}} / \varepsilon_{\mathrm{i}}\right)= \begin{cases}\tilde{\mathrm{y}} /|\bar{y}| & \text { if } \mid \hat{y} \geq \varepsilon_{\mathrm{i}} \\ \tilde{y}_{\mathrm{i}} / \varepsilon_{\mathrm{i}} & \text { if } \mid \tilde{\mathrm{y}}<\varepsilon_{\mathrm{i}}\end{cases}
$$

2) Set the gain matrix $K$ as:

$$
\begin{aligned}
& \mathrm{K}=\mathrm{D} \rho \\
& \rho=\operatorname{diag}\left(\rho_{1}, \ldots, \rho_{\mathrm{m}}\right), \rho_{\mathrm{i}} \geq 1
\end{aligned}
$$

3) Choose the width of boundary layer for $\widetilde{y}_{i}$ called $\varepsilon_{i}$, and which coincides with the saturation limit $\varepsilon_{\mathrm{i}}$, in the sat-function defined by (B.25); define the matrix

$$
\Delta=\operatorname{diag}\left(\varepsilon_{1}, \varepsilon_{2} \ldots \varepsilon_{\mathrm{m}}\right)
$$

The choice of $\varepsilon_{\mathrm{i}}$ is arbitrary, to some extent, but one might want to have it as small as possible as long as the particular choice does not conflict with the next steps.

4) Design for boundedness inside the boundary layer. Choose the gain matrices $H$ and $\rho$ such that for all $t \in R+$ and for all $\omega \in R$ :

$$
\begin{gathered}
\max \left[\sigma_{\max }\left(\mathrm{C}\left(\mathrm{j} \omega \mathrm{I}-\mathrm{A}+\left(\mathrm{H}+\mathrm{K} \Delta^{-1}\right) \mathrm{C}\right)^{-1} \mathrm{D}\right]+\right. \\
+\sigma_{\max }\left(\mathrm{C} \mathrm{e}^{\left(\mathrm{A} \cdot\left(\mathrm{H}+\mathrm{K} \Delta^{-1}\right) \mathrm{Ct}\right)} \max \left(\widetilde{\mathrm{x}}_{\mathrm{o}}\right) \leq \min _{\mathrm{i}}\left(\varepsilon_{\mathrm{i}}\right)\right.
\end{gathered}
$$

In practice this test has to be verified in the time interval of interest, typically during the initial transient. This method may be very conservative, as the usual singular analysis is.

5) Check whether the state estimation error bounds are within desirable 
limits:

$$
|\widetilde{\mathbf{x}}(\mathrm{t})| \leq \max _{0<\omega<+\infty}\left[\sigma_{\max }\left(\left(j \omega I-A+\left(\mathrm{H}+\mathrm{K} \Delta^{-1}\right) \mathrm{C}\right)^{-1} \mathrm{D}\right]\right.
$$

Clearly, if the transfer function matrix

$$
\mathrm{G}(\mathrm{s})=\mathrm{C}\left(\mathrm{s} \mathrm{I}-\mathrm{A}+\left(\mathrm{H}+\mathrm{K} \Delta^{-1}\right) \mathrm{C}\right)^{-1} \mathrm{D}
$$

has finite transmission zeros then the desired convergence time and the desired accuracy can be difficult to achieve. In particular, if the zeros turn out to be in the closed right half plane, then the problem may become particularly difficult, even impossible in some cases.

6) Check for stability outside the boundary layer (single measurement systems). The error dynamics can be written as:

$$
\begin{aligned}
\tilde{\mathbf{y}} & =\mathrm{C} \widetilde{\mathbf{x}} \\
\dot{\widetilde{\mathbf{x}}} & =(\mathrm{A}-\mathrm{H} \mathrm{C}) \widetilde{\mathbf{x}}-\mathrm{K} \phi \tilde{\mathbf{y}}
\end{aligned}
$$

It is necessary to verify that (A.8) is stable for $\phi$ such that $\phi$ is constrained in the sector:

$$
0<\phi<\frac{1}{\varepsilon}\left(1+\frac{1}{\rho}\right)=\bar{G}
$$

For instance, if the circle criterion is used, then it is sufficient to show that the Nyquist Plot of the transfer function

$$
\mathrm{H}_{f}(\mathrm{~s})=\mathrm{C}(\mathrm{sI}-\mathrm{A}+\mathrm{HC})^{-1} \mathrm{~K}
$$

is to the right of the vertical line that intersects the real axis at $-\frac{1}{\overline{\mathrm{G}}}$

7) Check for stability outside the boundary layer (systems with multiple measurements). The matrices $\mathrm{H}$ and $\mathrm{K}$ must be such that:

- ( A - H C - K M C ) has eigenvalues in the open left half plane; the matrix $M$ is a diagonal matrix whose entries are $m_{i i}=\frac{1}{2 \varepsilon_{i}}\left(1+\frac{1}{\rho_{i}}\right)$ 
- The following condition is verified for all values of $\omega \in \mathcal{R}$ at which $\mathrm{H}_{1}^{-1}$ exists:

$$
\sigma_{\min }\left(\left[M+H_{1}^{-1}(j \omega)\right] M^{1}\right) \geq 1
$$

where $\mathrm{H}_{1}(\mathrm{~s})=\mathrm{C}(\mathrm{sI}-\mathrm{A}+\mathrm{HC})^{-1} \mathrm{~K}$

8) If this last condition is satisfied, then the design is complete. Otherwise, one has to iterate changing the width of boundary layers and the choice of matrices $D, \rho$ and $H$.

\section{B.4 Alimov's Transformation}

The sliding observer error dynamics without disturbance is:

$$
\dot{\mathbf{x}}=\mathrm{A}_{\mathrm{m}} \mathrm{x}-\mathrm{K} 1_{\mathrm{s}}(\widetilde{\mathrm{y}})
$$

where $K$ is a $n x 1$ column matrix whose elements are positive and $1_{s}(s)$ is a signum function.

With a nonsingular Hurwitz system matrix $A_{m}$, we are investing the effect of a discontinuous switching surface.

$$
\mathrm{s}(\mathrm{t})=\widetilde{\mathrm{y}}=\mathrm{Cx}(\mathrm{t})
$$

We denote by $x_{+}\left(x^{0}, t\right)$ and $x_{-}\left(x^{o}, t\right)$ the trajectories of the systems

$$
\begin{aligned}
& \dot{\mathrm{x}}=\mathrm{f}_{+}(\mathrm{x})=\mathrm{Ax}-\mathrm{K}, \\
& \dot{\mathrm{x}}=\mathrm{f}_{-}(\mathrm{x})=\mathrm{Ax}+\mathrm{K}
\end{aligned}
$$

passing for $t=0$ through the initial point $x^{0}$ of the space $x$.

For the studying of the system, it is convenient to rewrite (B.39) in a new coordinate [Alimov, 1960] $\mathrm{x}_{+}=\mathrm{x}-\mathrm{A}^{-1} \mathrm{~K}, \mathrm{x}=\mathrm{x}+\mathrm{A}^{-1} \mathrm{~K}$ :

$$
\left\{\begin{array}{l}
\dot{\mathrm{x}}_{+}=\mathrm{Ax} \mathrm{x}_{+}, \\
\dot{\mathrm{x}}_{-}=\mathrm{A} \mathrm{x_{- }},
\end{array}\right.
$$


We denote by $\dot{\mathrm{s}}_{+}(\mathrm{x})$ and $\dot{\mathrm{s}}_{-}(\mathrm{x})$ the derivatives with respect to time of the function $\mathrm{s}(\mathrm{x})=\mathrm{Cx}(\mathrm{t})$ according to the system.

$$
\begin{aligned}
& \dot{\mathrm{s}}_{+}(\mathrm{x})=\mathrm{CA \textrm {x }}-\mathrm{C} \mathrm{K} 1_{\mathrm{s}}(\mathrm{s}) \\
& \dot{\mathrm{s}}_{-}(\mathrm{x})=\mathrm{CA} \mathrm{x}+\mathrm{CK} 1_{\mathrm{s}}(\mathrm{s})
\end{aligned}
$$

Switching plane $=$ Sliding zone + Passing zone

1) In the space $\mathrm{s}(\mathrm{x})>0$, we assume that $\mathrm{x}\left(\mathrm{x}^{\mathrm{o}}, \mathrm{t}\right)=\mathrm{x}_{+}\left(\mathrm{x}^{\circ}, \mathrm{t}\right)$, (for $\mathrm{s}(\mathrm{x})<0$, $\left.\mathbf{x}\left(\mathrm{x}^{\mathrm{o}}, \mathrm{t}\right)=\mathrm{x}_{-}\left(\mathrm{x}^{\mathrm{o}}, \mathrm{t}\right)\right)$.

2) At any point $\sigma_{\mathrm{t}}$ of the set $\mathrm{s}(\mathrm{x})=0, \dot{\mathrm{s}}_{+}(\mathrm{x}) \dot{\mathrm{s}}_{-}(\mathrm{x})>0$ which represents two half-planes $(\mathrm{C} \mathrm{K})^{-1} \mathrm{C} \mathrm{Ax}<-1, \mathrm{C} \mathrm{x}=0$ and $\left(\mathrm{C} \mathrm{K}^{-1} \mathrm{C} \mathrm{Ax}>+1, \mathrm{C} \mathrm{x}=0\right.$, the trajectories $x_{+}\left(\sigma_{t}, t\right), x\left(\sigma_{t}, t\right)$ go through the surface $s(x)=0$ in the same direction. We determine $\mathrm{x}\left(\sigma_{\mathrm{t}}, \mathrm{t}\right)$ by connecting continuously at $\sigma_{\mathrm{t}}$ those halftrajectories $x_{+}\left(\sigma_{t}, t\right)$ and $x_{-}\left(\sigma_{t}, t\right)$ which lie close to $\sigma_{t}$ in the regions $s(x)>0$ and $\mathrm{s}(\mathrm{x})<0$, respectively.

3) At any point $\sigma_{\mathrm{s}}$ of the set $\mathrm{s}(\mathrm{x})=0, \dot{\mathrm{s}}_{+}(\mathrm{x}) \dot{\mathrm{s}}_{-}(\mathrm{x})<0$, the trajectories $\mathrm{x}_{+}\left(\sigma_{\mathrm{s}}, \mathrm{t}\right)$ and $x_{-}\left(\sigma_{s}, t\right)$ go through the surface $s(x)=0$ in the opposite direction. The hyperplane is:

$$
\mathrm{C} \mathrm{x}=0 \text { and }-1<(\mathrm{C} \mathrm{K})^{-1} \mathrm{C} \mathrm{A} \mathrm{x}<+1
$$

\section{Example B.2}

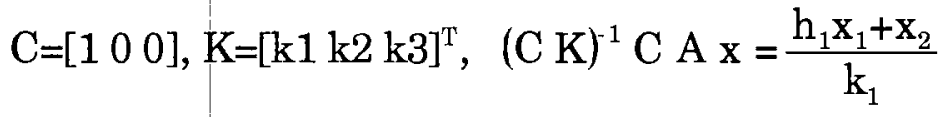
i.e. $-\mathrm{k}_{1}<\mathrm{x}_{2}<\mathrm{k}_{1}$, for $\mathrm{s}=0\left(\left|\mathrm{x}_{1}\right|<<1\right)$

The representative point of the system moves according to the equations

$$
\dot{\mathrm{x}}=\mathrm{Ax}-\mathrm{K} 1_{\mathrm{s}}(0)=\mathrm{Ax}-\mathrm{K} \zeta(\mathrm{t})
$$

where $\zeta(\mathrm{t})$ is chosen so that the derivative with respect to time $\dot{\mathrm{s}}_{\mathrm{o}}(\mathbf{x})$ of the function $\mathbf{s}(\mathbf{x})$ is obtained as in the above equation. It satisfies the equality 


$$
\dot{s}_{0}(x)=C A x(t)+C K \zeta(t)=0
$$

Combine (B.42) and (B.43), we obtain

$$
\begin{aligned}
& \mathrm{s}(\mathrm{x})=\mathrm{C} \mathrm{x}=0, \\
& \dot{\mathrm{x}}=\mathrm{f}_{\mathrm{o}}(\mathrm{x})=\mathrm{R} \mathrm{x}, \quad \mathrm{R}=\mathrm{A}-(\mathrm{C} \mathrm{K})^{-1} \mathrm{KCA}
\end{aligned}
$$

For the trajectories of the sliding dynamics, we will use the notation $x_{0}\left(x^{0}, t\right)$.

By using the equation (B.44), we can extend the definition of a switching function.

$$
1_{s}(s)=\left\{\begin{array}{r}
-1 \text { for } s<0, \\
\zeta \text { for } s=0 \\
+1 \text { for } s>0
\end{array}\right.
$$

The essence of Filippov's equivalent dynamics is precisely the definition of the discontinuous right hand side, including infinite valued functions, continuous in the same sense as the extended definition. The fact that the solution $\mathrm{x}\left(\mathrm{x}^{\mathrm{o}}, \mathrm{t}\right)$ can be continued for $\mathrm{t} \Rightarrow+\infty$, its uniqueness, and the property for are obvious; the continuity relative to $\mathrm{x}^{\circ}$ and $t$ can easily be proved by using (B.41) and(B.44). The solution $\mathrm{x}\left(\mathrm{x}^{\mathrm{o}}, \mathrm{t}\right)$ of the system (B.44), determined as above, is also a generalized solution of this system in the sense of the definition of Filippov.

We consider finally the boundary $\mathrm{s}(\mathrm{x})=0, \dot{\mathrm{s}}_{+}(\mathrm{x}) \dot{\mathrm{s}}(\mathrm{x})=0$ of the zone of the sliding regime, represented by the two hyperlines

$$
\begin{aligned}
& \mathrm{Cx}=0, \dot{\mathrm{s}}_{+}(\mathrm{x})=\mathrm{CAx}-\mathrm{CK} 1_{\mathrm{s}}(\mathrm{s})=0 \\
& \mathrm{Cx}=0, \dot{\mathrm{s}}_{-}(\mathrm{x})=\mathrm{CAx}+\mathrm{CK} 1_{\mathrm{s}}(\mathrm{s})=0
\end{aligned}
$$


We will form the Lyapunov function $\mathrm{V}(\mathrm{x})$ for the system with Hurwitz system matrix by linking on the surface $s(x)=0$. The Lyapunov functions:

$$
\begin{aligned}
& \mathrm{V}_{+}(\mathrm{x})=\mathrm{X}_{+} \mathrm{PX}_{+}, \mathrm{x}_{+}=\mathrm{x}-\mathrm{A}^{-1} \mathrm{~K} \\
& \mathrm{~V}_{-}(\mathrm{x})=\mathrm{X} \mathrm{PX}, \mathrm{x}_{-}=\mathrm{x}+\mathrm{A}^{-1} \mathrm{~K}
\end{aligned}
$$

For the systems ( $\mathrm{P}$ is a symmetric matrix of the nth order), respectively:

$$
V(x)= \begin{cases}V_{+}(x)-V_{+}(0) & \text { for } s(x) \geq 0 \\ V_{-}(x)-V_{-}(0) & \text { for } s(x) \leq 0\end{cases}
$$

The continuity of $V(x)$ is established if $V_{+}(x)$ and $V_{-}(x)$ are chosen so that the equality

$$
\mathrm{V}_{+}(\mathrm{x})-\mathrm{V}_{+}(0)=\mathrm{V}_{-}(\mathrm{x})-\mathrm{V}_{-}(0) \text { for } \mathrm{s}(\mathrm{x})=0
$$

holds, since the relation $\mathrm{V}_{+}(\mathrm{x})-\mathrm{V}_{+}(0)=\mathrm{V}(\mathrm{x})-\mathrm{V}(0)$ is equivalent to $x^{\prime} P A^{-1} K=0$. The connecting equation (i.e., so-called matching condition) can be rewritten in the form

$$
P A^{-1} K=\rho C^{T} \quad(\rho=\text { const } \neq 0)
$$

It is evident from the relation

$$
V(x)=x^{T} P x+2 \rho S 1_{s}(s)
$$

that follows from (B.53) and (B.55). If the function $x^{T} H x$ that is satisfying the condition (B.55) is positive definite, then the function $V(x)$ will also be positive definite for $\rho>0$.

For any solution $\mathbf{x}\left(\mathrm{x}^{0}, \mathrm{t}\right)$ everywhere except possibly at points of intersection of $x\left(x^{0}, t\right)$ with the surface $s(x)=0$, the derivative $\dot{V}(x)$ with respect to time of the function along $x\left(x^{0}, t\right)$ exists and satisfies the relations 
where

$$
\dot{V}(x)= \begin{cases}\dot{V}_{+}(x)=x_{+}^{T}\left(P A+A^{T} P\right) x_{+} & \text {for } s(x)>0, \\ \dot{V}_{(}(x)=x_{+}^{T}\left(P A+A^{T} P\right) x_{.} & \text {for } s(x)<0, \\ \dot{V}_{0}(x)=x^{T}\left(P R+R^{T} P\right) x & \text { for } s(x)=0, \dot{s}_{+}(x) \dot{s}_{-}(x)<0\end{cases}
$$

Let the $Q$ matrix is a symmetric positive definite matrix. Then, with the Hurwitz system matrix, there exists a unique matrix $P$ by the equation. It is apparent that the function $\dot{V}_{+}(x)$ or $\dot{V}_{-}(x)$ is negative definite and the function $V(x)$ decreases along any trajectory $x_{+}\left(x_{+}{ }^{\circ}, t\right)$ or $x_{-}\left(x_{-}{ }^{o}, t\right)$. Finally, we need to show the $\mathrm{V}(\mathrm{x})$ also decreases in sliding mode. 


\section{APPENDIX C \\ SIGN EQUALIZATION OF PASSING STATES \\ C.1 Transient State of the Reaching Dynamics}

At the passing instance, even though the velocity field is discontinuous, the trajectory of the solution point in the reaching domain is continuous. Consequently the dynamical characteristic for the half domain can be extended to the whole domain directly. Hence let us review the reaching dynamics in the view point of a linear system theory as follows:

$$
x(t)=e^{A(t-\tau)} x(\tau)+\int_{\tau}^{t} e^{A(t-\lambda)} B u(\lambda) d \lambda
$$

Zero-input response $\underbrace{}_{\text {zero-state response }}$

A very important property of any linear system is that the responses of the system can be decomposed into two parts, as follows:

$$
\begin{aligned}
& \text { Responses due to }\left\{x\left(t_{0}\right), u\left(t_{0}, \infty\right)\right\} \\
& =\text { responses due to }\{x(t o), 0\}+\text { responses due to }\left\{0, u\left(t_{0}, \infty\right)\right\}
\end{aligned}
$$

The responses due to $\left\{x\left(t_{0}\right), 0\right\}$ are called the zero-input response or a transient term: they are generated exclusively by the nonzero initial state $x\left(t_{0}\right)$. The responses due to $\left\{0, u\left(t_{0}, \infty\right)\right\}$ are called as the zero-state response or the forced term: they are excited exclusively by the input $u\left(t_{0}, \infty\right)$. Hence, for linear systems, we can consider the zero-input response and the zero-state 
responses independently. Reset the initial time zero at the passing instance and rewrite the equation (2.17):

$$
x(t)=e^{A t} x^{i}+e^{A t} \int_{0}^{t} e^{-A \lambda} B u(\lambda) d \lambda
$$

The disturbance input $u_{d}$ of the reaching dynamics is composed of the disturbance $w$ and $-\mathrm{k}_{\mathrm{n}} \operatorname{sign}\left(\mathrm{x}_{1}\right)$. If the disturbance input can be assumed as a white noise (or normal distributed random noise with zero mean) then the disturbance output of a high order system is much smaller than the output by the switching term $-\mathrm{k}_{\mathrm{n}} \operatorname{sign}\left(\mathrm{x}_{1}\right)$. Hence, the transient response for the step input is useful to understand the reaching dynamics.

\section{Transient responses of 2 order systems}

The transient response of the reaching dynamics may be described in terms of the rise time $t_{r}$ which is the time for the step response to reach from 10 to 90 percent of its final value, the exact values can again be obtained directly from the simulated results. The approximate relation between the rise time versus $\zeta$ is known for the 2-order system [Kuo, 1982]. For the range of $0<\zeta<1$, the rise time approximated by the first order equation is

$$
\mathrm{t}_{\mathrm{r}} \cong \frac{0.8+2.5 \zeta}{\mathrm{w}_{\mathrm{n}}}(0<\zeta<1)
$$

The rise time approximated by the second order equation is

$$
\mathrm{t}_{\mathrm{r}} \equiv \frac{1+1.1 \zeta+1.4 \zeta^{2}}{\mathrm{w}_{\mathrm{n}}}
$$

For the overdamped range $(1<\zeta)$, the rise time is approximately [Friedland, 1986]: 
$t_{r}=\frac{2 \zeta}{w_{n}}$

Transient responses of higher order systems

Some more detail descriptions of the transient response of the 2-order system can be found in the book of Clark [1962]. However, it is hard to find a paper which explicitly mentions the complex phenomena of the transient response for a higher order system including the zero input response. From the general solution (C.2), both terms have the same transient factor $\mathrm{e}^{\lambda t}$ so that the transient times of the step input response and zero input response should be the same order. With the special initial condition, the specific eigenmode only will be excited.

\section{Example C.1}

Consider the third order sliding observer reaching error dynamics:

$$
\left\{\begin{array}{l}
\dot{\mathrm{x}}_{1}=\mathrm{x}_{2 \mathrm{~s}}-\mathrm{h}_{1} \mathrm{x}_{1} \\
\dot{\mathrm{x}}_{2 \mathrm{~s}}=\mathrm{x}_{3 \mathrm{~s}}-\mathrm{h}_{2} \mathrm{x}_{1} \\
\dot{\mathrm{x}}_{3 \mathrm{~s}}=-\mathrm{h}_{3} \mathrm{x}_{1}+\mathrm{u}_{\mathrm{d}}
\end{array}\right.
$$

where $H=\left[\begin{array}{lll}.8 & .31 & .05\end{array}\right]^{\mathrm{T}}, \mathrm{w}=0$, initial states: $\mathrm{x}_{1}: 0, \mathrm{x}_{2}: 3, \mathrm{x}_{3}: 5, \mathrm{u}_{\mathrm{d}}=-\operatorname{sgn}\left(\mathrm{x}_{1}\right)$

The simulation results are shown in the Figure C.1. As it is expected, the transient time of the noised disturbance input is approximately the same as the step response. The transient time to for the zero input response can be defined as the time for the initial state to reach from 10 to 90 percent of its equilibrium state. The transient time $t_{t}$ can be obtained directly from the simulated results Figure C.1. The transient time is approximately 10 second for all. Figure C.1 (d) is the superposition of Figure C.1 (a) and (b). 

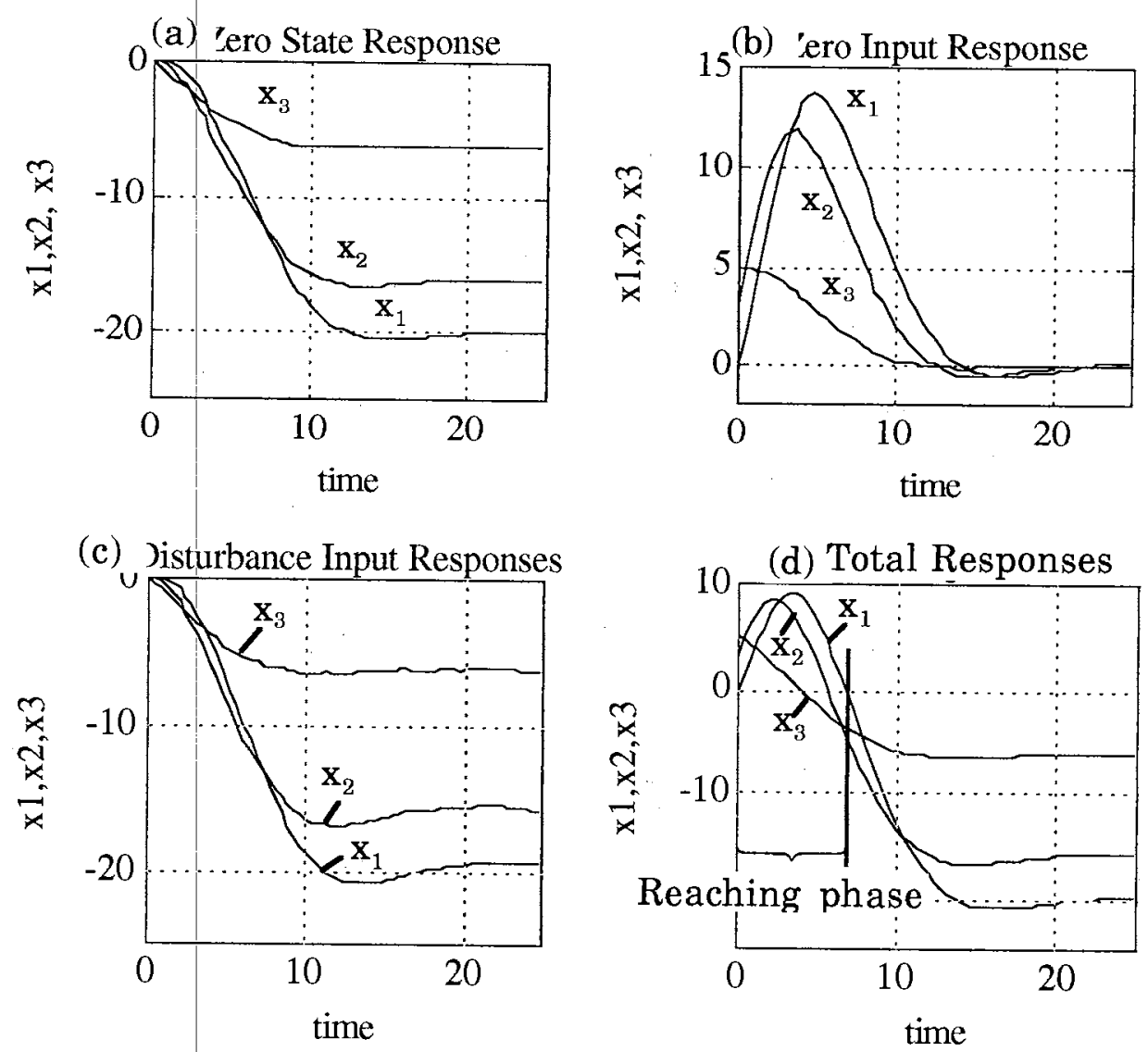

Figure C.1 Transient responses of reaching dynamics

a) Zero state response (Step input) b) Zero input response

c) Random noise disturbance input response d) Total response

\section{$\underline{\text { Conjecture C.1 }}$}

For the zero input response, the last state $x_{3}$ is a strictly decreasing function and reaches the minimum value when the state $x_{1}$ is zero. If all the initial states are positive at the hyperplane $\left(x_{1}=0\right)$, then all of the initial velocity field is positive except $\dot{x}_{n}$. The conceptual diagram is shown in Figure C.2. In the Figure C.2, the reaching time of $x_{n}$ is less than the reaching time of $x_{1}$. 
Approximate Zero Input Responses

1st Order Approximate Zero State Responses

Approximate

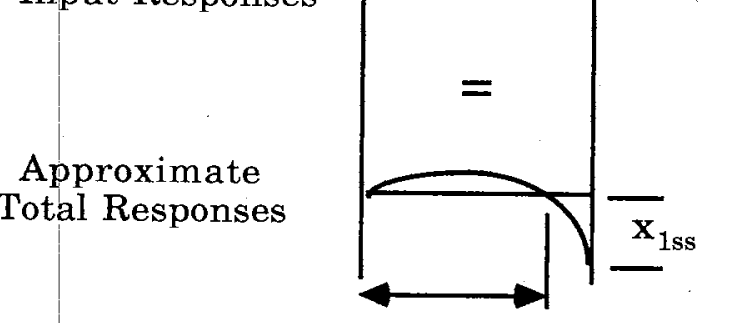

Reaching time
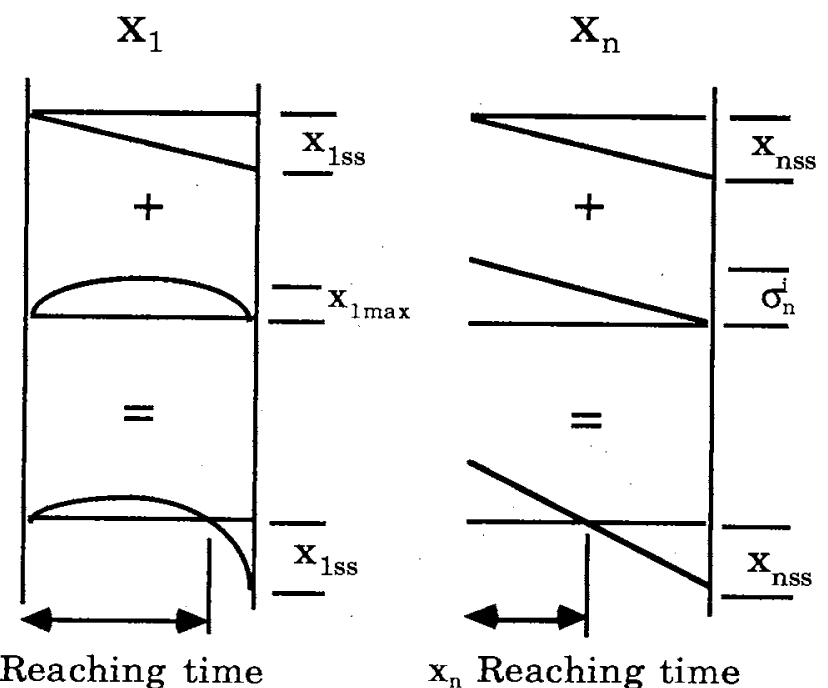

$\mathrm{x}_{\mathrm{n}}$ Reaching time

Figure C.2 The conceptual diagram of the reaching time

\section{Minimum Phase Transfer Function}

The transfer functions for the each state are

$$
\begin{aligned}
& x_{2 s}(s)=\left(s+h_{1}\right) x_{1}(s) \\
& x_{3 s}(s)=\left(s^{2}+h_{1} s+h_{2}\right) x_{1}(s) \\
& \ldots \\
& x_{n s}(s)=\left(s^{n-1}+h_{1} s^{n-2}+\ldots+h_{n-1}\right) x_{1}(s) \\
& u_{d}(s)=\left(s^{n}+h_{1} s^{n-1}+\ldots+h_{n}\right) x_{1}(s)
\end{aligned}
$$

The transfer functions between the state are

$$
\left(\begin{array}{rl}
\frac{x_{1}(s)}{x_{2 s}(s)} & =\frac{1}{\left(s+h_{1}\right)} \\
\frac{x_{2 s}(s)}{x_{3 s}(s)} & =\frac{\left(s+h_{1}\right)}{\left(s^{2}+h_{1} s+h_{2}\right)} \\
\cdots & \\
\frac{x_{(n-1) s}(s)}{x_{n s}(s)} & =\frac{\left(s^{n-2}+h_{1} s^{n-3}+\ldots+h_{n-2}\right)}{\left(s^{n-1}+h_{1} s^{n-2}+\ldots+h_{n-1}\right)} \\
\frac{x_{n s}(s)}{u_{d}(s)} & =\frac{\left(s^{n-1}+h_{1} s^{n-2}+\ldots+h_{n-1}\right)}{\left(s^{n}+h_{1} s^{n-1}+\ldots+h_{n}\right)}
\end{array}\right.
$$


Even though the reaching dynamics cannot be settle down to its steady state, the ratio between the steady states are helpful to understand the characteristic of the reaching dynamics. Apply the final value theorem and we get:

$$
\left\{\begin{array}{c}
\lim _{s \rightarrow 0} \frac{x_{1}(s)}{x_{2 s}(s)}=\frac{1}{h_{1}} \\
\lim _{s \rightarrow 0} \frac{x_{2 s}(s)}{x_{3 s}(s)}=\frac{h_{1}}{h_{2}} \\
\cdots \\
\lim _{s \rightarrow 0} \frac{x_{(n-1) s}(s)}{x_{n s}(s)}=\frac{h_{n-2}}{h_{n-1}} \\
\lim _{s \rightarrow 0} \frac{x_{n s}(s)}{u_{d}(s)}=\frac{h_{n-1}}{h_{n}}
\end{array}\right.
$$

For the transfer function between the input and output state is

$$
\lim _{s \rightarrow 0} \frac{x_{1}(s)}{u_{d}(s)}=\frac{1}{h_{n}}
$$

A transfer function whose poles and zeros all lie in the left-half s-plane is called a minimum phase transfer function. The steady state value of the nonminimum phase system is negative for the case of a simple transfer function that has a zero and a pole. If any of the coefficient $h_{i}$ is negative then some of the final value ratio of equation should be negative so that some the zero state responses move to different sign direction. Therefore the superposition of the zero state responses and zero input responses does not guarantee the sign equalization of all states $x_{2}, \ldots, x_{n}$ at the final passing point.

\section{C.2 Eigen structure}

The characteristic equation is

$$
\left|\lambda I-A_{m}\right|=\lambda^{n}+h_{1} \lambda^{n-1}+\ldots+h_{n-1} \lambda+h_{n}
$$




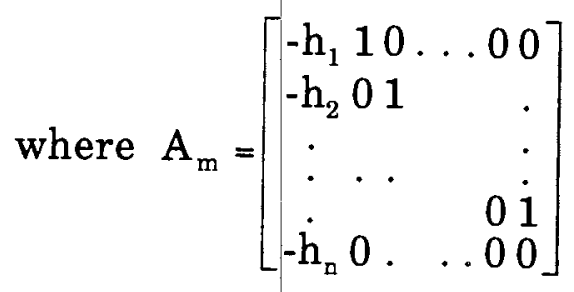

The Routh-Hurwitz stability method provides an answer to the question of stability by considering the characteristic equation of the system.

$$
\begin{aligned}
& \left(\lambda-\lambda_{1}\right)\left(\lambda-\lambda_{2}\right) \ldots\left(\lambda-\lambda_{n}\right) \\
& =\lambda^{n}-\left(\lambda_{1}+\lambda_{2}+\ldots+\lambda_{n}\right) \lambda^{n-1}+\left(\lambda_{1} \lambda_{2}+\ldots+\lambda_{n} \lambda_{1}\right) \lambda^{n-2}+\ldots+(-1)^{n}\left(\lambda_{1} \lambda_{2} \ldots \lambda_{n}\right)
\end{aligned}
$$

By comparing the two equations, we obtain the coefficients:

$h_{1}=(-1)^{1}$ (sum of all the eigenvalues)

$h_{2}=(-1)^{2}$ (sum of the products of the eigenvalues taken 2 at a times)

$h_{n}=(-1)^{n}$ (products of all $n$ eigenvalues)

For the canonical form system, the eigen structure has special form as follows:

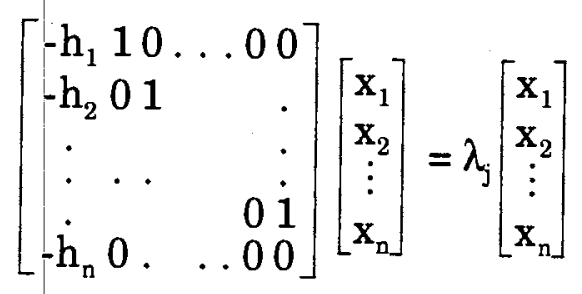

For $\lambda=\lambda_{j}$ case $(j=1,2, \ldots, n)$

From the 1st equation of (C.10), the eigenvector $x_{2}$ is obtained as follows:

$$
\begin{aligned}
x_{2} & =h_{1} x_{1}+\lambda_{j} x_{1} \\
& =\left\{-\left(\lambda_{1}+\lambda_{2}+\ldots+\lambda_{n}\right)+\lambda_{j}\right\} x_{1}
\end{aligned}
$$


From the following equations of equation (C.10), the remaining eigenvectors are also expressed as function of the state $\mathrm{x}_{1}$ and the eigenvalues:

$$
\begin{aligned}
x_{3} & =h_{2} x_{1}+\lambda_{j} x_{2} \\
& =\left\{\left(\lambda_{1} \lambda_{2}+\ldots+\lambda_{n} \lambda_{1}\right)-\lambda_{j}\left(\lambda_{1}+\lambda_{1}+\ldots+\lambda_{n}-\lambda_{j}\right)\right\} x_{1} \\
& =\left\{\sum_{k \neq j, l \neq j, k \neq 1} \lambda_{k} \lambda_{1}\right\} x_{1} \\
x_{4} & =h_{3} x_{1}+\lambda_{j} x_{3} \\
& =\left\{-\left(\lambda_{1} \lambda_{2} \lambda_{3}+\ldots+\lambda_{n} \lambda_{1} \lambda_{2}\right)+\lambda_{j}\left(\lambda_{1}+\lambda_{1}+\ldots+\lambda_{n}-\lambda_{j}\right)\right\} x_{1} \\
& =\left\{-\left(\sum_{m \neq n \neq 0} \lambda_{m} \lambda_{n} \lambda_{0}\right)+\lambda_{j} \sum_{k \neq j, l \neq j, k \neq 1} \lambda_{k} \lambda_{1}\right\} x_{1} \\
& =-\sum_{k \neq j, l \neq j, m \neq j,} \lambda_{k} \lambda_{1} \lambda_{m l} x_{1} \\
\cdots & x_{k \neq l \neq m} \\
x_{n} & =\frac{-h_{n}}{\lambda_{j}} x_{1}=(-1)^{n+1} \frac{\left(\lambda_{1} \lambda_{2} \ldots \lambda_{n}\right)}{\lambda_{j}} x_{1}
\end{aligned}
$$

If all the eigenvalues are negative, all the coefficient of the above equations are positive. Therefore, all the eigenvector have the same sign as $\mathbf{x}_{1}$.

If all eigenvalues of $A$ are distinct, the response of $\dot{x}=A x$ due to $x(0)=x_{0}$ can be written as:

$$
\widehat{x}(s)=(s I-A)^{-1} x_{o}=\sum_{j} \frac{1}{s-\lambda_{j}} q_{j} p_{j} x_{o}(C .13) \text { where } q_{j} \text { and } p_{j} \text { are, }
$$

respectively, right and left eigenvector of the system matrix A which is associated with $\lambda_{j}$. In the time domain, the equation becomes:

$$
x(t)=\sum_{j}\left(p_{j} x_{o}\right) e^{\lambda_{j i} t} q_{j}
$$

If $x_{o}$ is chosen so that $\mathbf{p}_{j} \mathbf{x}_{\mathbf{o}}=0$ for all $j$ except $j=i$, then the equation (C.14) 
diminishes to

$$
x(t)=\left(p_{i} x_{o}\right) e^{\lambda_{i} t} q_{i}
$$

For this initial state, only the mode $\mathrm{e}^{\lambda_{\mathrm{i}} \mathrm{t}}$ is excited and $\mathrm{x}(\mathrm{t})$ will travel along the direction of the eigenvector $\mathrm{q}_{\mathrm{i}}$.

\section{C.3 3-order System}

Rewrite a 3-order right reaching dynamics:

$$
\left\{\begin{array}{c}
\dot{x}_{1}=-h_{1} x_{1}+x_{2+} \\
\dot{x}_{2+}=-h_{2} x_{1}+x_{3+} \\
\dot{x}_{3+}=-h_{3} x_{1}+u_{d}
\end{array}\right.
$$

where $u_{d}=w-k_{n} \operatorname{sgn}\left(x_{1}\right)$

Since the state $x_{1}$ is not changed by the coordinate transformation in each domain $\Omega_{+}, \Omega_{\mathrm{o}}, \Omega_{-}$, it is noted without a sign of + or - . It is obvious that, in the shifted coordinate, the above system is a linear system and it is asymptotically stable and attractive to the shifted origin. Since the right half domain $\Omega+$ and the left half domain $\Omega$ - are symmetric to each other about the origin of original coordinate, let us consider the half domain $\Omega+$ only. If the initial states are not on the sliding patch, then the solution point needs to be attracted to the sliding patch. Hence, for the stability analysis purpose, it is assumed that the initial point is satisfying the passing condition $\sigma_{2+}^{i}>0$ without loss of generality.

\section{Case 1)}

With the initial condition $\sigma_{3_{+}}^{\mathrm{i}}>0$, the initial velocity field of the reaching dynamics is obtained from (C.15): 


$$
\left\{\begin{array}{l}
\dot{\mathbf{x}}_{1}^{i}>0 \\
\dot{\mathbf{x}}_{2+}^{i}>0 \\
\dot{\mathbf{x}}_{3+}^{i}<0
\end{array}\right.
$$

We can do pole placement to choose the linear correction coefficients. Let us assume that the system is underdamped or critically damped. Since the Hurwitz system is asymptotically stable, the velocity of $x_{1}$ is negative as it approaches the switching plane. The velocity field, $\dot{x}_{1}^{\mathrm{f}}<0$, is due to the state $\mathrm{x}_{2}$ in the equation (C.15).

$$
\text { Hence, } \dot{\mathrm{x}}_{1}^{\mathrm{f}}<0 \text { implies } \sigma_{2+}^{\mathrm{f}}<0
$$

where $\left\{\begin{array}{l}\text { if } 0>\sigma_{2+}^{f}>-2 \mathrm{k}_{1}: \text { satisfy sliding condition } \\ \text { if } \sigma_{2+}^{\mathrm{f}}<-2 \mathrm{k}_{1}: \text { passing the hyper plane }\end{array}\right.$

The initial passing point with $\sigma_{3+}^{\mathrm{i}}>0$ arrived at final point, $\sigma_{2+}^{f}<0$. The associated velocity field is $\dot{x}_{2+}=-h_{2} x_{1}+x_{3+}$ in which the first term $-h_{2} x_{1}$ is always negative and $x_{3+}$ is strictly decreasing. Consequently, the direction of velocity $\dot{\mathbf{x}}_{2+}$ can be changed only once and the velocity of $\mathbf{x}_{2+}$ is negative at the final passing time.

$$
\sigma_{2+}^{f}<0 \text { implies } \dot{\mathbf{x}}_{2+}^{\mathrm{f}}<0
$$

The approching velocity $\dot{\mathrm{x}}_{2+}^{\mathrm{f}}<0$ near the switching plane $\mathrm{x}_{1} \approx 0$ is due to the negative state of $x_{3+}$.

$$
\dot{\mathbf{x}}_{2_{+}}^{\mathrm{f}}<0 \text { implies } \sigma_{3+}^{\mathrm{f}}<0
$$

The equation (C.17) and (C.19) shows that both of the final passing state are negative $\left(\sigma_{2+}^{f}<0\right.$ and $\left.\sigma_{3+}^{f}<0\right)$. The general shearing effect of the sliding observer can be explained by the "sign equalization" of the final passing state. 
Let us consider the other case $\sigma_{3+}^{\mathrm{i}}<0$.

$\underline{\text { Case 2) }}$

With the initial condition $\sigma_{3+}^{\mathrm{i}}<0$, the initial velocity field of the reaching dynamics is

$$
\begin{aligned}
& \dot{\mathbf{x}}_{1}^{i}>0 \\
& \dot{\mathbf{x}}_{2+}^{i}<0 \\
& \dot{\mathbf{x}}_{3+}^{i}<0
\end{aligned}
$$

With the initial passing point $\sigma_{3+}^{i}<0$, the final state is negative because the $\dot{\mathrm{x}}_{3}$ is strictly decreasing.

$$
\dot{\mathbf{x}}_{3+}^{\mathrm{i}}<0 \text { implies } \sigma_{3+}^{\mathrm{f}}<0
$$

The approaching velocity $\dot{\mathrm{x}}_{1+}^{\mathrm{f}}<0$ near the switching plane $\mathrm{x}_{1} \approx 0$ is due to the negative of state $x_{2+}$.

$$
\dot{\mathbf{x}}_{1+}^{\mathrm{f}}<0 \text { implies } \sigma_{2+}^{\mathrm{f}}<0
$$

We can see here the "sign equalization" of the states of the final passing point as it is in the equation (C.21) and (C.22). For the case of passing right, the unified sign of final passing state is positive $\left(\sigma_{2-}^{f}>0 \sigma_{3-}^{f}>0\right)$. The sign equalization is due to the characteristic of the sliding observer velocity field. The direction of each state $\dot{x}_{j}$ is the function of $x_{1}$ and $x_{j+1}$ (for the last state: $u_{d}$ ) only. Since, in the right reaching domain, $x_{1}$ is positive only and the velocity of last state $\dot{x}_{3+}$ is strictly decreasing, each direction sign can change only once or not at all. This special feature of the sliding observer guarantees the sign equalization in 2- and 3-order cases.

It is useful to note that $\sigma_{2 \mathrm{~s}}^{\mathrm{f}}$ of the final passing point has the opposite sign of $\sigma_{2 s}^{i}$ : 


$$
\sigma_{2 \mathrm{~s}}^{\mathrm{i}} \sigma_{2 \mathrm{~s}}^{\mathrm{s}}<0 \quad(\mathrm{~s}=+ \text { or }-)
$$

When the solution point passes left, the succeeded initial state in the shifted-coordinate is obtained by the equation (2.60). Particularly the second state determines that the solution point pass through the hyperplane or not.

$$
\left\{\begin{array}{c}
\sigma_{1}^{i}\left(\tau_{j}\right)=\sigma_{1+}^{f}\left(\tau_{j}\right) \\
\sigma_{2-}^{j}\left(\tau_{j}\right)=\sigma_{2+}^{f}\left(\tau_{j}\right)+2 k_{1} \\
\cdots \\
\sigma_{n-}^{i}\left(\tau_{j}\right)=\sigma_{n+}^{f}\left(\tau_{j}\right)+2 k_{n-1}
\end{array}\right.
$$

If the sign of the second state in the following shifted-coordinate does not change, then the solution point crosses the hyperplane. If the sign is changed, then the sliding motion starts because the solution point moves to the hyperplane from the both sides.

\section{C.4 High Order System}

In the previous sections, the transient and the final states were reviewed. For the minimum phase LTI systems, the sign of final values are unity as shown in the previous section. The eigen structure of the reaching dynamics shows sign equalization also. However, since the reaching dynamics is only some part of the transient period, it is a subtle problem to prove the sign equalization of the reaching dynamics.

\section{Conjecture C.1 Initially unified sign case.}

If the initial signs are the same each other then the sign of the velocity changes only once.

Justification) Consider a n-order right reaching dynamics in the shifted- 
coordinate.

$$
\left\{\begin{array}{c}
\dot{x}_{1}=-h_{1} x_{1}+x_{2+} \\
\dot{x}_{2+}=-h_{2} x_{1}+x_{3+} \\
\cdots \\
\dot{x}_{n+}=-h_{n} x_{1}+u_{d}
\end{array}\right.
$$

Let assume the sign of all of the initial states are positive except $x_{1}=0$. At the hyperplane $\left(x_{1}=0\right)$, all of the velocity field are positive direction except the last velocity.

Let assume the sign of $\dot{x}_{1}$ changes only once then the state $x_{1}$ is convex over the right reaching domain as followings:

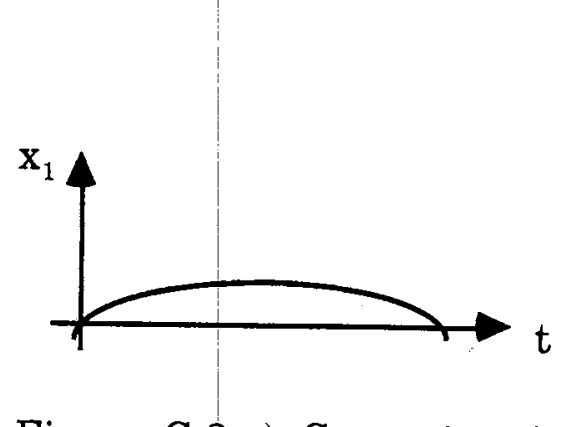

Figure C. 3 a) Convexity of $x_{1}$

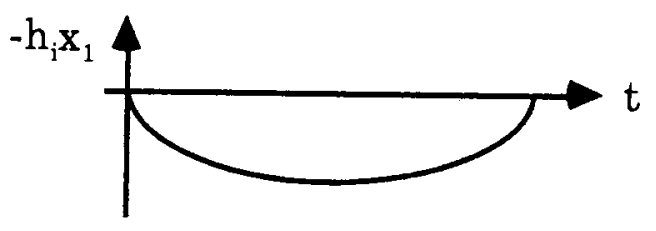

b) Concavity of $-h_{i} x_{1}$

For the i_th equation, the first term in the RHS, $-h_{j} x_{1}$, is concave over the right-reaching domain. The last state $x_{n}$ that is initially positive decreases strictly and the sign of the final passing point of $x_{n}$ is negative (see detail Chapter C.1).

The initial velocity direction of the second last state, i.e., $\dot{\mathbf{x}}_{(\mathrm{n}-1)+}$ is positive and becomes negative when $h_{n-1} x_{1}$ is greater than the state $x_{n}$. Since the last state $x_{n}$ strictly decreases and the term, $-h_{n-1} x_{1}$, is concave over the time domain, the sign of $\dot{\mathbf{x}}_{(\mathrm{n}-1)+}$ changes only once. Therefore the second last state, $x_{(n-1)+}$ is concave. Let assume the state decreases enough so that it 
becomes negative as plotted in Figure $\mathrm{C}$ a).

The initial velocity of the third last state, i.e., $\dot{\mathrm{x}}_{(\mathrm{n}-2)+}$ is positive and becomes negative when $h_{n-2} x_{1}$ is greater than the state $x_{n-1}$. Since the second last state $x_{n-1}$ convex and the term, $-h_{n-2} x_{1}$, is concave over the whole time domain, the sign of $\dot{x}_{(\mathrm{n}-2)+}$ changes only once. Therefore the third last state, $\mathrm{x}_{(\mathrm{n}-2)+}$ is concave. The sign change of the rest velocity can be explained as the same way as the former ones. Therefore, the assumption of convexity of $x_{1}$ is valid.

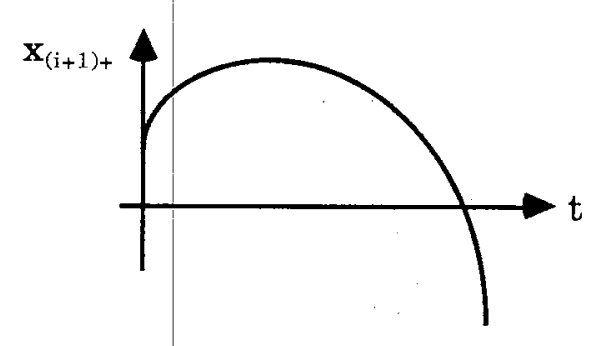

Figure C.4 a) Convexity of the state

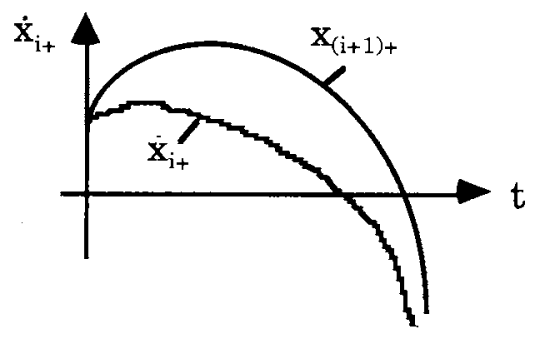

b) Sign Change of the state

Each state decreases enough to be negative as the state $x_{1}$ approaches to the hyperplane. If the state $x_{2}$ is positive when the state $x_{1}$ decreases and approaches to the hyperplane, then the velocity $\dot{x}_{1}$ is positive and the state $x_{1}$ increases again. Therefore, $x_{1}$ cannot be zero with the positive state $x_{2}$ and this is contradict to the convexity of $x_{1}$.

\section{Conjecture 2}

All the final signs are changed from the initial sign

Justification) If any state $x_{i+}$ has the same sign at the approaching the hyperplane instance then it will change the sign of velocity of the previous 
state $\dot{\mathbf{x}}_{(\mathrm{i}-1)+}$ again. This is contradiction to the Lemma 1)

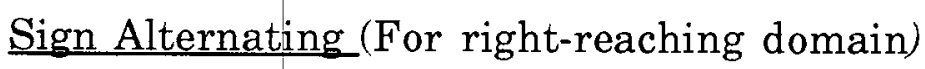

Rewrite the reaching dynamics:

$$
\dot{\mathbf{x}}_{++}=-\mathrm{Hx}_{1}+\left(\begin{array}{c}
\mathrm{x}_{2+} \\
\mathrm{x}_{3+} \\
\cdots \\
\mathrm{x}_{\mathrm{n}+} \\
\mathrm{u}_{\mathrm{d}}
\end{array}\right)
$$

where $H=\left[h_{1} h_{2}, \ldots, h_{n}\right]^{T}$

The initial velocity field is function of $\sigma_{+}^{i}$ and $u_{d}$ only. The signs of the initial state are

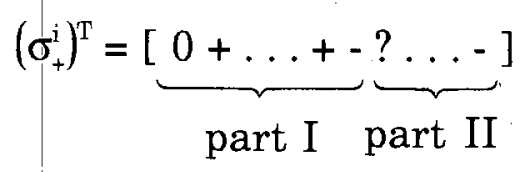

The $j$-th equation of the reaching dynamics has the first negative state $\mathbf{x}_{j+1}:$

$$
\dot{\mathbf{x}}_{j+}=-\mathrm{h}_{\mathrm{j}} \mathrm{x}_{1}+\mathrm{x}_{(\mathrm{j}+1)+}
$$

Part I) If $x_{j+1}$ is negative all the time until $x_{1}$ become 0 , the first negative state $x_{j+1}$ do the same role as $u_{d}$ in the initial sign unity case. Hence, the number $\mathrm{N}_{\mathrm{I}}$ is increased at least 1 . If $\mathrm{x}_{j+1}$ become positive at the instance $\mathrm{x}_{1}$ arriving the hyperplane then the $\mathrm{N}_{\mathrm{I}}$ does not change in the next reaching domain.

Part II) The disturbance input also unifies the sign of the state in descending order so that the system will satisfy the sliding condition or on the way to the sign unity of the passing point.

\section{C.5 Numerical Search Program}




\section{C.5 Numerical Search Program}

The sourse code of the MATLAB program SOON and the SIMNON program Reaching are listed for the third order case only because the extension to the higher order system is straight forward.

\section{C.5.1 SOON (for MATLAB)}

\begin{tabular}{|c|c|}
\hline$\%$ & SOON.M \\
\hline $\begin{array}{l}\% \\
\% \\
\% \\
\% \\
\% \\
\%\end{array}$ & $\begin{array}{l}\text { Sliding } \text { Observer design by wQ Qrst reaching dynamics } \\
\text { for Nonlinear/ uncertain system } \\
\text { PURPOSE: Known Bounded Disturbunce and } \\
\text { Known Bounded Initial States } \\
\text { Design the sliding observer coefficients in order to } \\
\text { converge to the sliding patch directly. }\end{array}$ \\
\hline
\end{tabular}

$\%$ For 3rd order only

odr=input('System Order=')

$\mathrm{w}=$ input('W $\max =$ ')

$\mathrm{w} 11=1.1 * \mathrm{w}$

$\mathrm{k} 3=\max (1.1, \mathrm{w} 11)$;

\% Select the proper Linear Coefficients

disp('Choose $\mathrm{H}$ so that the system is critically damped or slightly under damped');

h $1=$ input('h $1=')$

$\mathrm{h} 2=$ input('h2=')

h3=input('h3=')

\% Save data for the Simnon program Reaching

save d_reaching

!ren d_reaching.mat d_reaching.t

$\%$ Run the Simnon Program Reaching mreaching

$\%$ Get the Output of mreaching

!Ren o_reaching.t o_reaching.mat

load o_reaching

$\%$ Steady State with constant disturbance

$\mathrm{x} 2 \mathrm{ss}=\mathrm{w}^{*} \mathrm{k} 1 / \mathrm{k} 3$

$\mathrm{x} 3 \mathrm{ss}=\mathrm{w}^{*} \mathrm{k} 2 / \mathrm{k} 3$; 
\%disp('Sliding dynamics e.v.');

$\% \mathrm{As}=[-\mathrm{k}(2,1) / \mathrm{k}(1,1) 1 ;-\mathrm{k}(3,1) / \mathrm{k}(1,1) 0]$;

$\mathrm{As}=[-\mathrm{k} 2 / \mathrm{k} 11 ;-\mathrm{k} 3 / \mathrm{k} 10]$;

eas=eig(As);

if re(eas) $>0$ then disp('Warning:Unstable Equivalent Dynamics!!')

subplot(122);grid;

$\operatorname{axis}\left(\left[\begin{array}{llll}-0.5 & 0.1 & -1.5 & 1.5]) \text {; }\end{array}\right.\right.$

plot (eas, '*');

xlabel('Re');ylabel('Im');

title('E.V. of sliding dynamics');

end

C.5.2 Reaching (for SIMNON)

MACRO M_REACHING

" Plot whole step at once : for third order system only syst sys3 cost3 gold3 conn3 "*3.t: 3rd order system store $x 1 \times 2$ x3 t [sys3] tau j[cost3] error $1 \mathrm{e}-6$

init x2in[gold3]:3 "The initial bound of the states

init $x 3$ in[gold3]:2

" Estimates the proper number of the evaluations of the cost function $" \quad$ in the GOLD.T search.

let plow $=0$

let phigh $=2$

let acc $=.01$

let unc $0 .=$ phigh-plow "Initial uncertainty.

let $\mathrm{fl} .=1$.

let $\mathrm{f} 2 .=1$.

free qf1.

free qf2.

free $x$. "Ratio $\mathrm{fn}-1 / \mathrm{fn}$.

free uncn. "Uncertainty after $\mathrm{N}$ evaluations.

free $\mathrm{n}$. "Required number of evaluations.

free p0. "Initial optimization starting point.

free teval. "Simulation time for evaluation.

free toptim. "Total simulation time.

default nmax. $=20$ "Maximal number of evaluations.

"-----Calculation of required number of evaluations

for $\mathrm{i}=1$. to $n \max$.

let $\mathrm{qf2}=\mathrm{f} 1 .+\mathrm{f} 2$.

let $\mathrm{qf1}=\mathrm{f} 2$.

let $\mathrm{fl} .=\mathrm{qf1}$.

let $\mathrm{f} 2 .=\mathrm{qf} 2$. 
let $\mathrm{x} .=\mathrm{f} 1 . / \mathrm{f2}$.

let uncn. $=$ unc $0 . / \mathrm{f} 1$.

let $\mathrm{n} . \mathrm{i}$

let nacc $=-.05$

if uncn. le nacc goto exit

next i

"-..-.

label exit

"-

let $\mathrm{p} 0 .=\mathrm{x}$. unc 0 .

let $\mathrm{p} 0 .=\mathrm{plow}+\mathrm{p} 0$.

init $\mathrm{p}: \mathrm{p} 0$.

init pmin:p0.

init phi:phigh

init plo:plow

disp teval/teval

let toptim. $=$ n. *teval.

let tperid. $=12 *$ teval.

simu 0 toptim. .001/dz31 tperid.

init x1in[gold3]:x1in[gold3]

init $x 2$ in[gold3]:x2in[gold3]

init x3in[gold3]:x3in[gold3]

init jmin[gold3]:1e33

init m[gold3]:m[gold3]

export $\mathrm{dz} 31<\mathrm{dz} 31$

let dat $=\mathrm{dz} 3$

" Step by step

default step. $=30$

for $\mathrm{jj}=2$ to step.

let datjj=dat+jj

let $\mathrm{p} 0 .=\mathrm{x} .{ }^{*}$ unc 0 .

let $\mathrm{p} 0 .=$ plow $+\mathrm{p} 0$.

let $n n .=j j * n$.

let $\mathrm{jj} 1=\mathrm{jj}-1$

let $\mathrm{nn} 0 .=\mathrm{jj} 1 * \mathrm{n}$.

disp teval/teval "from gold3

$\operatorname{disp} \mathrm{x} 1 / \mathrm{x} 1$

let toptim. $=$ nn. ${ }^{*}$ teval.

let tbgn. $=n n 0 . *^{*}$ teval.

simu tbgn. toptim. $0.0001 /$ datjj tperid.

init x1in[gold3]:x1in[gold3]

init x2in[gold3]:x2in[gold3] 


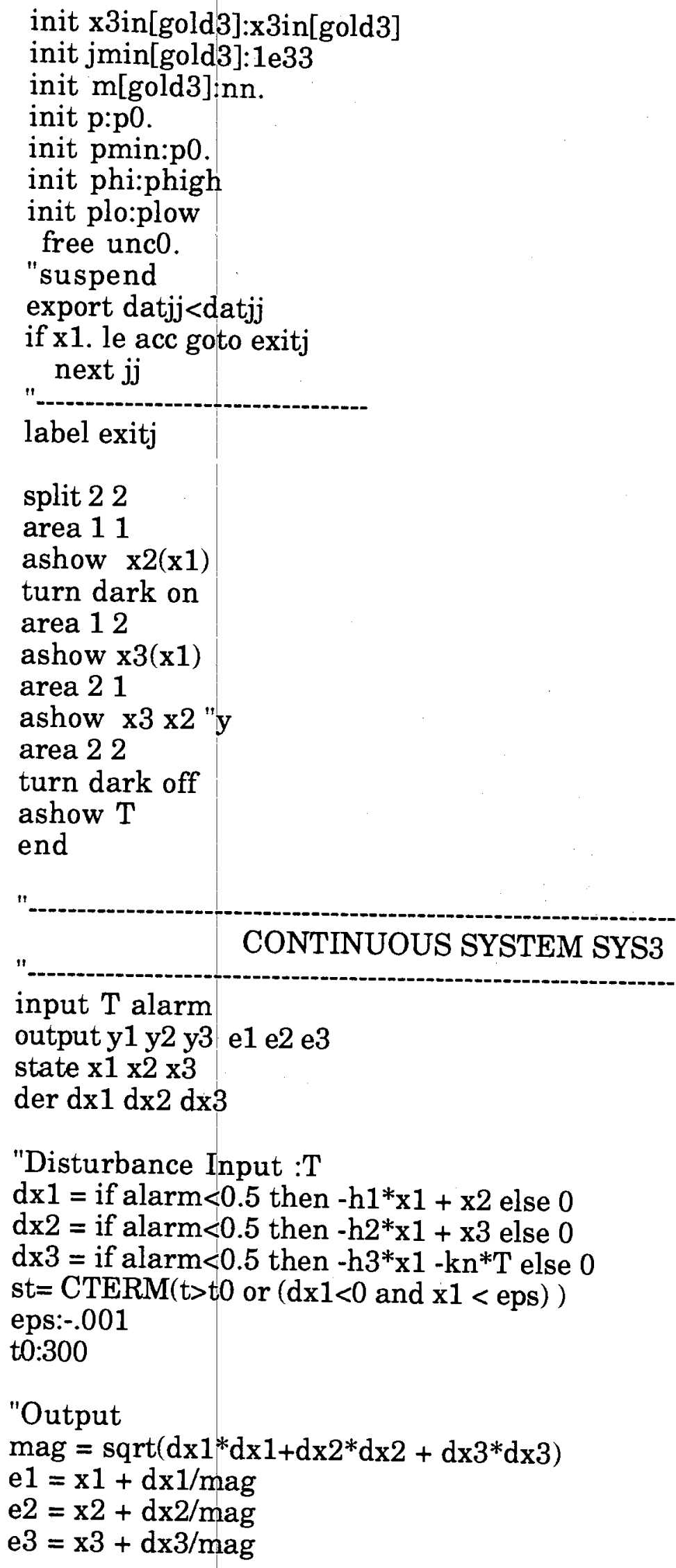




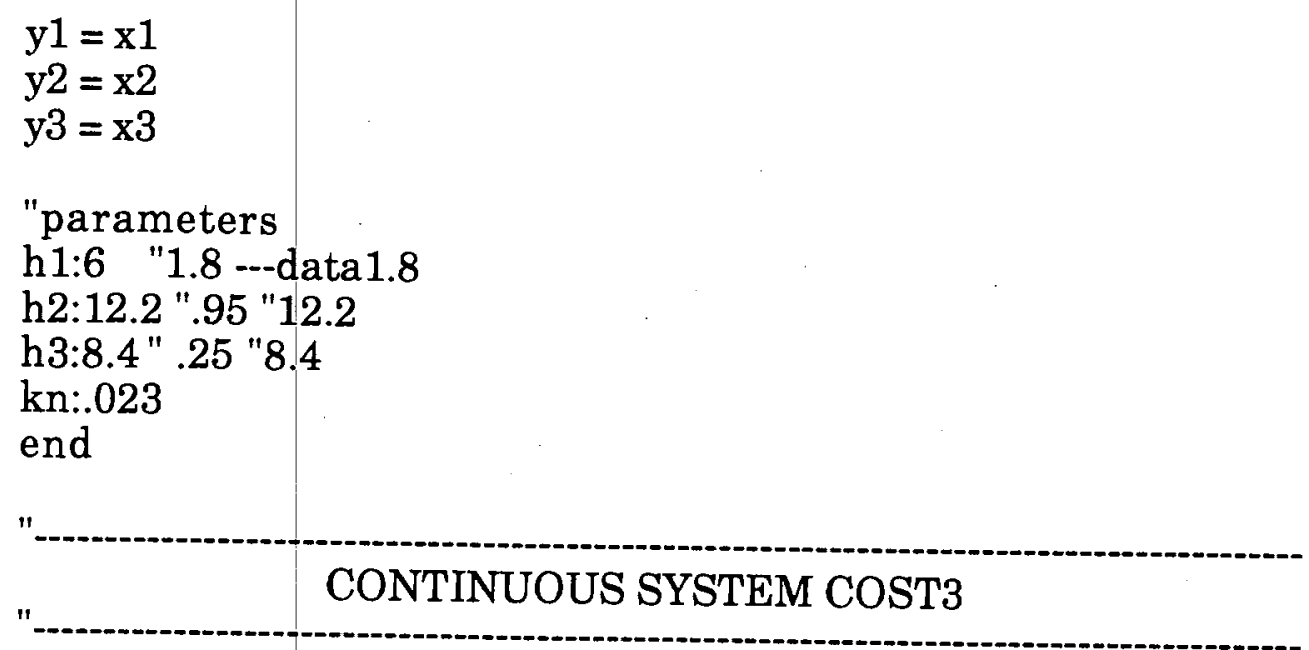

"Evaluates the cost function

input e1 e2 e3 tau

output $\mathrm{j}$ alarm

state $\mathrm{s} 1$

der ds 1

"Measure distance

$\mathrm{ds} 1=$ if not alarm then $\mathrm{e} 1^{*} \mathrm{e} 1+\mathrm{e} 2{ }^{*} \mathrm{e} 2+\mathrm{e} 3^{*} \mathrm{e} 3$ else 0

$\mathrm{v}=\mathrm{s} 1+.01$

$\mathrm{j}=1 / \mathrm{v}$

"Alarm test:

alarm $=$ if $j>$ jmax then 1 else 0

"parameters:

Jmax : 1000

end

"

\section{DISCRETE SYSTEM GOLD3}

"Discrete system to perform optimination of one parameter input j y1 y2 y3

output peval tbegin

state plo phi pmin $\mathrm{p}$ jmin $\quad \mathrm{x}$ 1in $\mathrm{x} 2$ in $\mathrm{x} 3 \mathrm{in} \mathrm{m} \mathrm{n}$ new qplo qphi qpmin qP qJmin qx1in qx2in qx3in qm qn

time $t$

tsamp ts

"Update the search state:

left $=\mathrm{P}<\mathrm{mid}$

decr $=\mathrm{J}<\mathrm{Jmin}$

stepn $=q m-q n$

$\mathrm{t} 15=\mathrm{stepn}+1.5$

t05 = stepn +0.5

PloFix $=$ if $\mathrm{t}<\mathrm{t} 15 *$ teval then 1 else $\bmod ($ left+decr $+1,2)$

PhiFix $=$ if $t<t 15 *$ teval then 1 else mod(left+decr,2) 
unc $=$ newPhi-newPlo "Uncertainty interval.

$\operatorname{mid}=(\mathrm{Phi}+\mathrm{Plo}) / 2$ "Midpoint of the interval

newPlo $=$ if PloFix then Plo else if decr then Pmin else P

newPhi $=$ if PhiFix then Phi else if decr then Pmin else $P$

newPmin $=$ if decr then $P$ else Pmin

$\mathrm{qJ} \min =$ if $\mathrm{t}>\mathrm{t} 05^{*}$ teval and decr then $\mathrm{J}$ else Jmin

$\mathrm{qPlo}=$ if $\mathrm{t}>\mathrm{t15} 5^{*}$ teval then newPlo else Plo

$\mathrm{qPhi}=$ if $\mathrm{t}>\mathrm{t} 15^{*}$ teval then newPhi else $\mathrm{Phi}$

$\mathrm{qPmin}=$ if $\mathrm{t}>\mathrm{t} 05^{*}$ tevalthen newPmin else Pmin

$\mathrm{qP}=$ Peval

"Calculate the new evaluation point Peval:

Peval $=$ if $t>t 05 *$ teval then newPhi+newPlo-newPmin else Pmin

"Reset process and loss-function after each test

$\mathrm{qm}=\mathrm{m}+1$ "Counter

$\mathrm{qn}=$ if $\mathrm{n}<11.5$ then $\mathrm{n}+1$ else 1 "Numbering in each step

$\mathrm{qx} 1 \mathrm{in}=$ if $\bmod (\mathrm{m}+1, \mathrm{nmax})>0$ then $x 1$ in else $\mathrm{y} 1$

$\mathrm{qx} 2$ in $=$ if $\bmod (\mathrm{m}+1, \mathrm{nmax})>0$ then $\mathrm{x} 2$ in else $\mathrm{y} 2$

qx3in $=$ if $\bmod (m+1, n \max )>0$ then $x 3$ in else $y 3$

$\mathrm{x} 1[$ sys 3$]=\mathrm{x} 1$ in

$\mathrm{x} 2[$ sys3] $=\mathrm{x} 2$ in

$\mathrm{x} 3[\mathrm{sys} 3]=\mathrm{x} 3 \mathrm{in}$

$\mathrm{s} 1[\cos \mathrm{t} 3]=0$

"New sample:

ts $=\mathrm{t}+$ teval

tbegin $=t$

"Initial values that should be set by the user

nmax:12 "Max number of evaluation in each step

$\mathrm{n}: 0$

$\mathrm{m}: 0$

Plo :0.0 "Lower bound of parameter

Phi :1.0 "Upper bound of parameter

$P$ : 0.61803 "Golden section ratio

"Jmin :1E33 "Max real value

teval : .05 "Simulation time for evaluation

end

time $\mathrm{t}$

$\mathrm{T}[$ sys3 $]=$ Peval[gold3 $]$

e1 [cost 3$]=$ e1[sys 3$]$

$\mathrm{e} 2[$ cost 3$]=\mathrm{e} 2[\mathrm{sys} 3]$

$\mathrm{e} 3[\operatorname{cost} 3]=\mathrm{e} 3[\mathrm{sys} 3]$

$\mathrm{y} 1$ [gold3] = y1[sys3] 


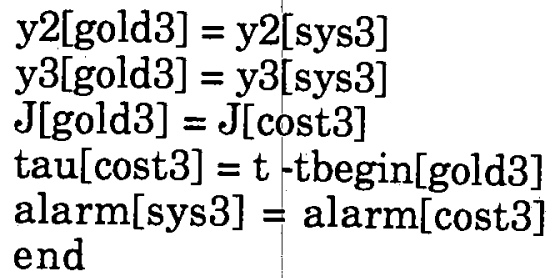




\section{VITA \\ JinHee Choi \\ Candidate for the Degree of \\ Doctor of Philosophy}

Thesis: A STUDY ON SLIDING MODE STATE ESTIMATION

Major Field: Mechanical Engineering

Biographical:

Personal Data: Born in Pusan, Korea, August 15, 1956, the son of Mr. and Mrs. JongKwan Choi.

Education: Received the Bachelor of Science degree in Mechanical Design and Production Engineering from Seoul National University in February, 1979; received the Master of Science degree in Mechanical Engineering from Korea Advanced Institute of Science and Technology in February, 1981; completed the requirements for the Doctor of Philosophy degree at Oklahoma State University in May, 1993.

Professional Experience: Lecturer, Korea Military Academy, June 1981 - February 1983; Instructor, Korea Military Academy, March 1983 - April 1986; Assistant Professor, Korea Military Academy, May 1986 - present. 